\title{
LICHENS OF
}

THE STATE OF WASHINGTON

by

GRACE E. HOWARD 



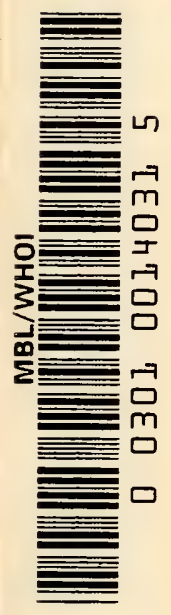



LICHENS OF THE STATE OF WASHINGTON 


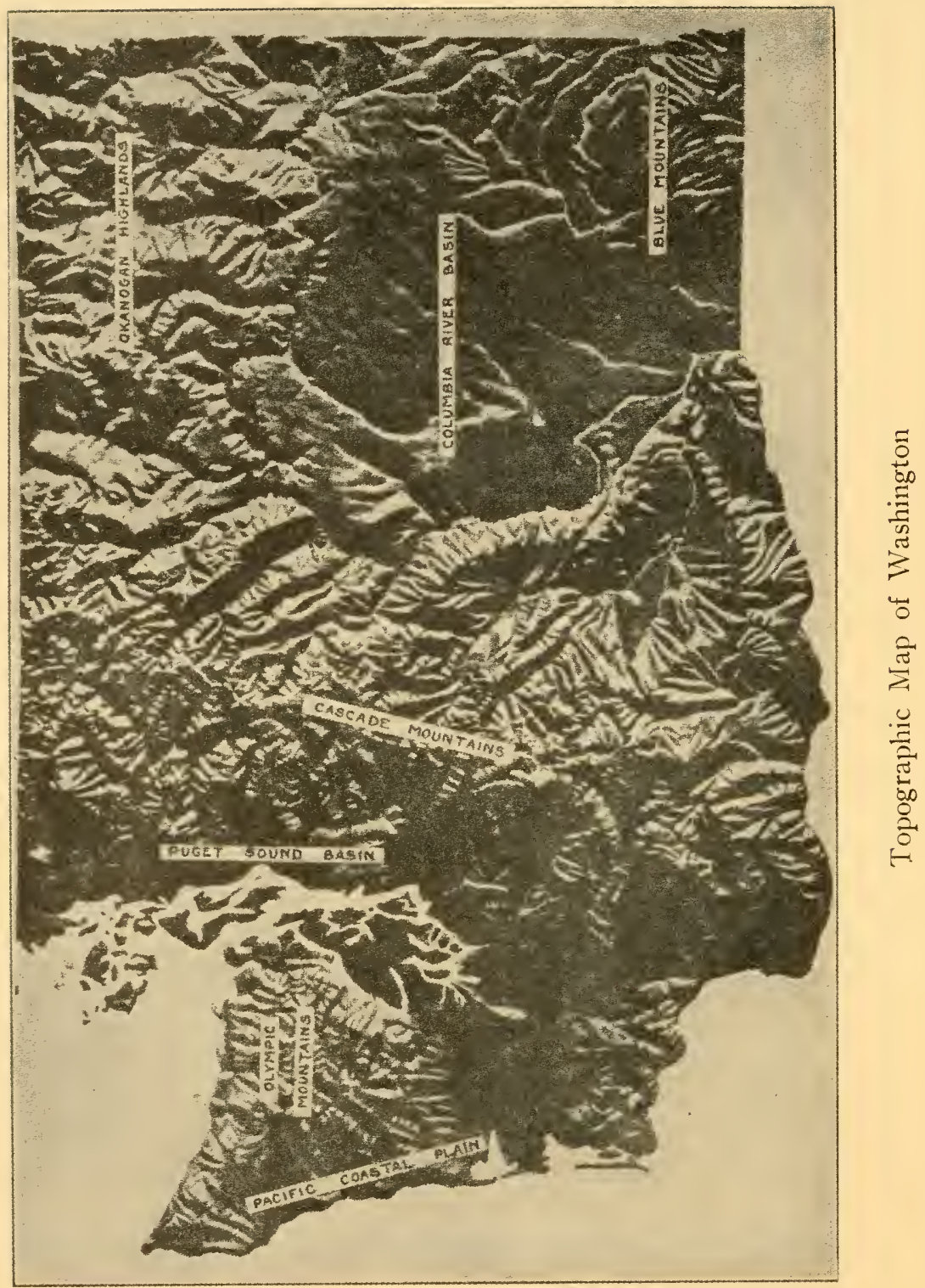




\section{Lichens of \\ the State of Washington}

BY

GRACE E. HOWARD

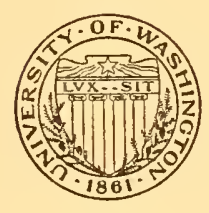

SEATTLE

UNIVERSITY OF WASHINGTON PRESS

1950 
PRINTED IN THE UNITED STATES OF ANERICA 


\section{To \\ Margaret C. Ferguson \\ AND}

Theodore C. Frye

"Among the mountains, the silver lichen-spots rest star-like on the stone; and the gathering orange stain upon the edge of yonder rock reflects the sunsets of a thousand years."

Ruskin, Modern Painters 



\section{PREFACE}

I began the study of lichens upon the advice of Emeritus Professor Margaret C. Ferguson of Wellesley College and Professor Theodore C. Frye of the University of Washington. Quite naturally my attention was soon focused on the lichens of Washington, since I grew up in the state, am a graduate of its university, and have always been interested in its flora. Since so little has been written about the lichens of the state of Washington, there seems to be a real need for this book. I did my first collecting in the state in the summer of 1928; my most extensive trip was during the summer and fall of 1931; further collections were made during the summers of 1930 , 1934, 1937, and 1938. Again in 1940, when I had a sabbatical year from Wellesley College, another extensive trip, lasting the entire summer, was taken, and additional collections were made in the summer of 1942.

I am by no means the first collector of lichens in the state; in one of my previous publications, Preliminary Report on the Lichens of the State of Washington (1937), the work of earlier collectors in the state has been reviewed. However, in that study no mention is made of the work of Wilhelm N. Suksdorf, who did an immense amount of collecting in Washington. Mr. Suksdorf, who was born in Germany in 1850 and died in Washington in 1932, was interested in both cryptogams and phanerogams, and records of his collections appear in various books and articles. Weber (1944) has given an interesting account of Suksdorf's botanical work. To the list of collectors must be added the name of Alexander H. Smith, who collected extensively in and near the Olympic National Park in 1935 and 1939. Hedrick (1942) published an annotated list of Smith's collections.

In this book 335 species and varieties, 69 genera, and 28 families are included. These constitute the commoner lichens of Washington, at least in the regions that have been thoroughly explored. No doubt there are many more species and varieties growing in the state that will be collected and described in the years to come. In the keys to species, I have attempted, as far as possible, to make use of the most evident and least questionable characteristics, and to avoid separations based on color and other poorly defined characters. I hope the distinguishing phrases or sentences characterizing many of the species and varieties will prove helpful. It is rather doubtful if anyone can construct keys that can be used successfully without access to a reliable herbarium.

In writing this book use has been made of the publications of many students, the most important being: Tuckerman, Synopsis of the North American Lichens, Pts. I and II $(1882,1888)$; Fink, The Lichens of Minnesota (1910); Herre, The Lichens of the Santa Cruz Peninsula, California (1910); Hasse, The Lichen Flora of Southern California (1913); 
A. L. Smith, A Monograph of the British Lichens (2d ed.), Pts. I and II (1918, 1926); Lindau, Die Flechten (1923); Zahlbruckner, Lichenes (1926); Sandstede, Die Grattung Cladonia (1931); Zahlbruckner, Catalogue Lichenum Universalis, 9 vols. (1921-1932) ; Frey, Familie Umbilicariaceae (1933); Fink, Lichen Flora of the United States (1935).

The Farlow Herbarium of Harvard University is very rich in lichens; among its most important and valuable American lichen herbaria are Tuckerman, Merrill, Riddle, Sprague, Taylor, and Robbins. Many Foster, Grant, and Herre specimens are deposited at Farlow; also Zahlbruckner types and many exsiccati from all over the world. The Farlow Herbarium has been used more than any other in the determination of specimens and for the obtainment of distributional data. The University Herbarium at the University of Michigan, where the large and important Fink Herbarium is deposited, has been consulted freely. The study of specimens from the Herbarium at the University of Washington has resulted in much interesting distributional data.

Much of the actual writing of this book has been done at Wellesley College. The Herbarium of Wellesley College contains many lichen types and valuable collections, and the library is unusually rich in lichen literature. For many years there has been a botanist at Wellesley especially interested in lichens. The first was Miss Clara E. Cummings, who was followed by Dr. Lincoln W. Riddle and Miss Mabel A. Stone. At the present time I am carrying on the tradition. We have all been responsible in some way for the extensive lichen herbarium and library at Wellesley College.

The value of color changes, brought about by certain chemical reactions, has long been recognized for the determination of lichens. In this book color reactions in combination with morphological characters have been used for the determination of certain lichens. However, these color reactions have not been used alone, as key characters. The chemicals used are paraphenylenediamine, potassium hydroxide, and calcium hypochlorite. In most tests, each reagent is applied separately, but in some potassium hydroxide is applied first and this is followed by calcium hypochlorite. A positive color reaction with one or more of these reagents indicates the presence of certain lichen acids. Iodine solution is used as a test for the presence of various carbohydrates.

All specimens were collected by the author, unless otherwise indicated. Most of the altitudes given, for specimens collected by me, were estimated from topographical maps published by the United States Department of the Interior, Geological Survey. No altitudes of less than 200 feet have been reported.

I have used the classification and nomenclature of Zahlbruckner, as given in his catalogue (1921-1934), unless otherwise indicated.

I wish to express my sincere appreciation of Dr. D. H. Linder (now deceased), former Curator of the Farlow Reference Library and Herbarium of Cryptogamic Botany of Harvard University, for the generous use of the facilities of the library and herbarium; and to Miss Hilda F. Harris, libra- 
rian of the Farlow Reference Library, for her assistance at all times. I am indebted to Mrs. Joyce H. Jones, Assistant Curator of the University Herbarium, University of Michigan, for the loan of herbarium specimens and for information concerning herbarium specimens; also to Dr. D. E. Stuntz of the University of Washington, for the loan of herbarium specimens from the Herbarium of the University of Washington.

Grace E. Howard

Wellesley College

July, 1946

Associate Professor of Botany 

CONTENTS

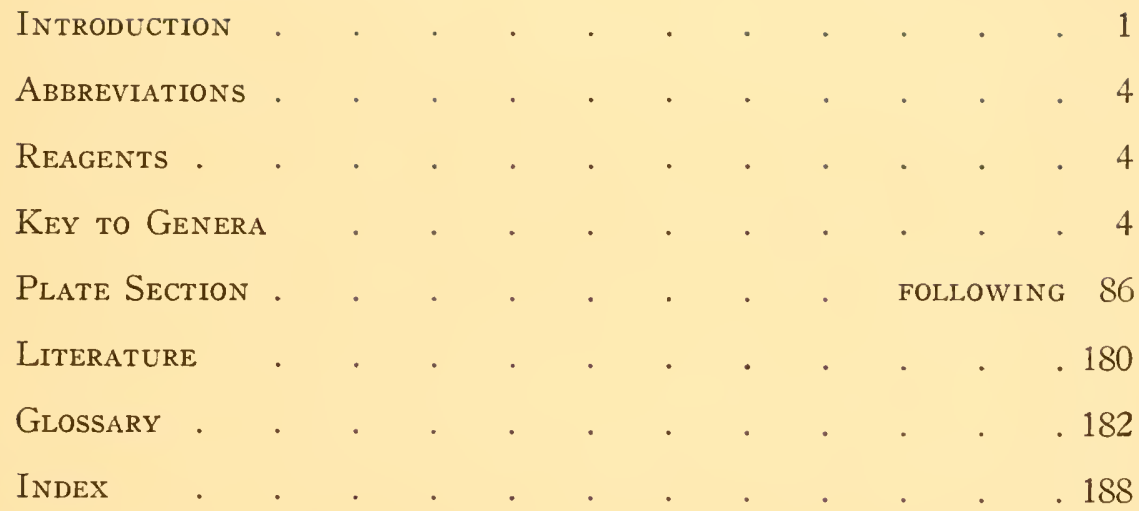





\title{
LICHENS OF THE STATE OF WASHINGTON
}

\section{By}

\author{
GRACE E. HOWARD
}

\section{INTRODUCTION}

Lichens belong to the Thallophytes, the lowest of the great divisions of the plant kingdom. A lichen consists of a fungus and an alga living together in a large degree of mutual benefit. The alga contains chlorophyll, which enables it to carry on photosynthesis and make carbohydrates for the partnership (consortium) ; the fungus absorbs both water and mineral nutrients and also synthesizes the proteins. Furthermore, the fungus determines the form, shape, size, and strength of the organism. In a few lichens the alga has been isolated and grown in pure culture; this has also been done with the fungus. However, it is doubtful if they ever become separated in nature. These algal lichens can be referred to present-day genera and often even to species; but the fungi cannot be placed, with any degree of certainty, in any of our present-day genera. Hence, the identity of the fungus seems to have been changed much more than that of the alga. In only three genera is the fungus a Basidiomycete; in all other genera it is an Ascomycete. The alga belongs either to the Cyanophyceae or to the Chlorophyceae.

It was believed by earlier students that the relationship between the alga and the fungus was parasitism; this view is still held by some students today. When a lichen spore germinates it produces only the fungal hyphae and not a lichen. Because of this fact it has been said that there are no true species of lichens. Before a lichen can develop, these fungal hyphae must grow around some algal cells, entangling them and penetrating their cell walls with haustoria, so that the fungus may be supplied with the necessary carbohydrates; furthermore, in some lichens the algae grow completely inside the fungal hyphae. It is true that dead algae have been reported within the lichen thallus, a point in favor of parasitism.

Other students consider this relationship to be a symbiosis. Even though the carbohydrates for a lichen are made by the algae, they receive in return an increased amount of light; they also receive water and proteins from the fungi. The algae are benefited greatly by the larger variety of habitats in which they are able to grow while living in this symbiotic state, since lichens have a wider distribution than any other group of plants. For these reasons, I consider this relationship a symbiosis. The continued healthy and sturdy growth of lichens, under adverse conditions, adds strength to this idea.

Based upon habit of growth, lichens are classified as crustose, foliose, and 
fruticose. In crustose types the thallus grows flat upon the substratum, often so closely attached that it is difficult or impossible to get it off without breaking the lichen. The thallus may be simply a tangled layer of interwoven hyphae and algae cells with no differentiation into layers, or there may be an upper pseudocortex with an algal layer below this and some indication of a medullary layer below the algal layer. Hyphal rhizoids probably penetrate the substratum, although it is difficult to find these in a cross section of the thallus. In some crustose species the thallus is entirely within the substratum. In foliose lichens the thallus is usually much larger, leaf-like as the name suggests, not so closely attached to the substratum, and usually divided into distinct layers. These layers are an upper dermis, an upper cellular cortex, an algal layer, a medullary layer, and a lower cellular cortex. However, some foliose lichens do not have all of these layers. In fruticose types the thallus grows at right angles to the substratum. These lichens may be either pendant, hanging from the branches of trees, or growing upright from the substratum. They are often slender and long, somewhat hair-like, more or less cylindrical in form. The thallus has a pseudocortex of interwoven hyphae, extending in the direction of the axis or at right angles to it; just inside is the algal layer and inside that the medullary layer, which may be well developed, forming a solid center, poorly developed, or scarcely developed at all, leaving a hollow center.

The fruit-bodies of lichens are either apothecia or perithecia. Apothecialbearing lichens are much more abundant than perithecial-bearing ones. An apothecium is a cup-shaped or saucer-shaped body surrounded by either a thalline or a proper margin (exciple) or by both. The epithecium, when present, is the topmost layer of the disk, very thin and scarcely distinguishable from the hymenium, which lies beneath. The hymenium, or thecium as it has been called by some students, consists of asci and paraphyses, and below it is the hypothecium. These margins (exciples) are very important key characters for the separation of genera. The thalline margin is continuous with the thallus, resembling it in structure, and always contains some of the same algae that are found in the thallus. When present, the proper margin lies just inside the thalline; it is an extension of the hypothecium, and hence more truly a part of the apothecium than is the thalline margin. A perithecium is an oval or spherical-shaped body, opening only by a small pore at the top, which produces ascospores and has a structure very similar to an apothecium.

As in free-living fungi, the ascospores produced in the fruit-bodies of lichens must be the result of sexuality somewhere in the life cycle. Moreover, both sex organs have been seen in a few lichens, but the entire cycle has not been satisfactorily worked out. No doubt the most common and valuable method of reproduction is by means of soredia. A soredium is a group of algal cells surrounded and held together by a mass of fungal hyphae. Masses of soredia have the appearance of powder on the upper surfaces of many lichens; they are often produced in large quantities and are disseminated by wind and rain. Often on the trunks of trees in the dense forests west of the Cascades there can be seen thick layers of soredia on the 
bark. Under proper environmental conditions these will grow into mature lichens, of the same species that produced them. Some species have never been found bearing fruit-bodies, so they must be reproduced either by soredia or fragmentation. It is known that fragmentation is a common type of multiplication of many kinds of lichens. Rejuvenescence takes place in a number of lichens, but it is especially noticeable in specimens of crustose and foliose forms, in which the center of the thallus mat has died and the periphery has remained alive. Here there may be seen new centers of growth arising at various points. This enables a single lichen to spread a considerable distance over the substratum. Rejuvenescence also takes place in the Cladonias, in which the basal portions of the podetia die and growth continues at the tops of the podetia.

The lichen flora of Washington represents a wide variety of forms, no doubt a result of the varied and interesting topography of the state. The Cascade Mountains divide Washington into an eastern and a western section. The western section, which comprises three-eighths of the state, consists of the Pacific Coastal Plain, the Olympic Mountains, and the Puget Sound Basin. Land elevations vary from sea level to an average of 6000 feet for the Cascades and nearly the same for the Olympics. Several peaks in the Cascades have an elevation of over 10,000 feet, while Mt. Rainier, the highest in Washington, is 14,408 feet. Both the Olympics and the Cascades have a great effect on many of the physiographic features of the state; however, the Japanese current is largely responsible for the mild temperatures on the ocean side of the Cascades. The prevailing winds which are from the ocean bring to this western section a marine climate characterized by considerable moisture, cool summers and comparatively mild winters. These moisture-laden winds, from the ocean, must first rise to pass over the Olympics and later over the Cascades; hence the precipitation is heavy on the windward slopes. Stations at sea level, on the windward side of the Olympics, have an average precipitation of 125 inches, and those on the leeward side less than twenty inches. The eastern section, comprising fiveeighths of the state, is all a part of the Columbia River Basin. In the northeast are the Okanogan Highlands, consisting of gently rolling hills, with a north and south trend, and an average elevation of 2000 feet, with some peaks rising to 6000 feet. In the extreme southeast are the Blue Mountains. Here the highest elevations are about 7000 feet. Since the prevailing winds that blow across the state come from the west and southwest, the air has lost most of its moisture after it has passed over both the Olympics and Cascades; consequently the air over all eastern Washington is dry most of the year. Here the precipitation varies from an annual average of six inches, in the lowest valleys, to more than sixty inches on the higher eastern slopes of the Cascades. In some of the central valleys, such as Wenatchee and Yakima, and also in the Great Bend Country and the Grand Coulee semidesert conditions prevail. For further information concerning the topography or physical framework of Washington the reader should consult Piper (1906), Martin and Corbin (1930), and Freeman and Martin (1942). 


\section{AbBreviations}

C-Cummings Herbarium, Wellesley College.

D-Private Lichen Herbarium, Dr. C. W. Dodge, St. Louis, Missouri.

F-Farlow Herbarium, Harvard University.

M-University Herbarium, University of Michigan.

S-Sprague Herbarium in Farlow Herbarium, Harvard University.

$\mathrm{T}$ - Tuckerman Herbarium in Farlow Herbarium, Harvard University.

W-Herbarium of Wellesley College.

WTC-Herbarium of the State College of Washington.

WTU-Herbarium of the University of Washington.

M. \& P.-Maerz, A., and M. R. Paul, A Dictionary of Color, 1930.

R-Ridgway, R., Color Standards and Color Nomenclature, 1912.

\section{REAGENTS}

Calcium hypochlorite- $\mathrm{CaOCl}_{2}$ (saturated aqueous solution).

Iodine solution-IKI ( 2 per cent aqueous solution of potassium iodide, with sufficient iodine added to color the solution a deep yellow).

Potassium hydroxide- $\mathrm{KOH}$ (25-50 per cent aqueous solution).

Paraphenylenediamine-P (fresh saturated alcoholic solution).

\section{Artificial Key to the Genera}

\section{CRUSTOSE LICHENS}

1. Podetia present (thallus usually twofold, consisting of a primary crustose thallus that often disappears, and a secondary thallus consisting of erect podetia).

2. Podetia short often poorly developed, usually simple.

3. Apothecia black.

39. Pilophoron

3. Apothecia light-colored.

38. Baeomyces

2. Podetia longer, well developed, usually branched.

4. Podetia solid, cephalodia common.

41. Stereocaulon

4. Podetia usually hollow, cephalodia rare.

40. Cladonia

1. Podetia absent.

5. Spores borne in a perithecium.

6. Spores hyaline, nonseptate.

6. Spores brown, muriform.

1. Verrucaria

2. Staurothcle

5. Spores borne in an apothecium.

7. Apothecia stalked.

8. Stalk long, well developed.

5. Calicium

8. Stalk short, sometimes lacking.

9. Thallus greenish, resembling a layer of paint; disk pale flesh color ( $R$ ) or rosy pink...

48. Icmadophila

9. Thallus grayish, disk dull black.

6. Cyphelium

7. Apothecia not stalked.

10. Apothecia borne in a stroma; disk usually black, sometimes white pruinose; spores 1-many septate.

8. Arthonia

10. Apothecia not borne in a stroma.

11. Apothecia lirella-form, always black.

12. Spores nonseptate; thallus entirely within the substratum. 9. Xylographa

12. Spores septate, thallus superficial.

13. Spores 3-many septate; paraphyses branched, interwoven. 11. Graphis 
13. Spores many-septate; paraphyses not branched, distinct.

11. Apothecia not lirella-form, variously colored.

10. Opegrapha

14. Apothecia immersed in warts often breaking into soredial masses.

14. Apothecia not as above.

45. Pertusaria

15. Thalline margin conspicuous, permanent.

16. Spores nonseptate.

17. Spores very small, asci often 100 -spored

17. Spores never very small.

44. Acarospora

18. Blue-green algal component.

22. Pannaria

18. Green algal component, very rarely a blue-green.

19. Paraphyses branched and entangled.

47. Ochrolechia

19. Paraphyses unbranched, distinct or coherent.

20. Asci normally 8-spored.

20. Asci normally more than 8-spored.

46. Lecanora

16. Spores transversely septate.

51. Candelariella

21. Spores hyaline.

22. Spores polar-bilocular.

22. Spores not polar-bilocular, 1-septate.

65. Caloplaca

23. Green algal component.

24. Disk some shade of yellow or orange.......51. Candelariella

24. Disk never yellow or orange.

23. Blue-green algal component.

49. Lecania

21. Spores brown, polar-bilocular

23. Massalongia 68. Rinodina

15. Thalline margin absent, or inconspicuous and soon disappearing.

25. Spores nonseptate.

26. Spores very small, asci many-spored.

26. Spores medium sized, asci 4-16-spored.

43. Biatorella

27. Blue-green algal component.

27. Green algal component.

21. Parmeliella

28. Thallus violet, purple, or red with $\mathrm{KOH}$

28. Thallus not colored as above with $\mathrm{KOH}$.

64. Blastenia

31. Lecidea

26. Spores large, more than $65 \mu$ long, asci $1-2$-spored.

32. Mycoblastus

25. Spores transversely septate.

29. Spores hyaline.

30. Spores polar-bilocular.

30. Spores not polar-bilocular.

64. Blastenia

31. Spores 1-septate.

32. Thallus poorly developed, sometimes disappearing within the substratum. 15. Microphiale

32. Thallus well developed never disappearing within the substratum.

33. Catillaria

31. Spores more than 1-septate.

33. Growing on rocks, soil, or mosses over rocks.

34. Disk blood-red or bright brownish red; epithecium deep purple or blue with $\mathrm{KOH}$ 50. Haematomma

34. Disk never any shade of red, usually black; epithecium never purple or blue with $\mathrm{KOH}$.

33. Growing on soil, moss, or trees. 35. Toninia

35. Spores with a gelatinous halo.

35. Spores without a gelatinous halo.

34. Bacidia

36. Disk flesh color $(\mathrm{R})$ or rosy red; hypothecium hyaline.

36. Disk dark brown or black, hypothecium Icmadophila 
black.

12. Lecanactis

29. Spores not hyaline, gray, or brown.

37. Spores with a distinct gelatinous halo.

37. Spores without a distinct gelatinous halo.

37. Rhizocarpon

25. Spores muriform.

38. Spores hyaline.

39. Usually growing on bark; numerous crater-like apothecia

13. Thelotrema

39. Not as above.

40. Always growing on rock; spores not more than $20 \mu$ long.......

16. Gyalecta

40. Growing on trees and encrusting mosses on rocks; spores more than $40 \mu$ long.

36. Lopadium

38. Spores brown.

41. Thalline margin disappearing early, spores without a gelatinous halo.

14. Diploschistes

41. Thalline margin absent entirely, spores with a distinct gelatinous halo.

37. Rhizocarpon

\section{SQUAMULOSE OR FOLIOSE}

1. Podetia present (thallus usually twofold, consisting of a primary squamulose or foliose thallus that often disappears, and a secondary thallus consisting of erect podetia).

40. Cladonia

1. Podetia absent.

2. Thallus more or less gelatinous when moist.

3. Thallus decidedly gelatinous when moist, brittle when dry; algal component Nostoc.

4. Both upper and lower cortices lacking

19. Collema

4. Both upper and lower cortices present or only the upper cortex present.

20. Leptogium

3. Thallus not so decidedly gelatinous when moist, brittle when dry; algal component Scytoncma.

18. Polychidium

2. Thallus not gelatinous when moist.

5. Spores borne in a perithecium.

6. Thallus umbilicate, rather large, foliose; spores hyaline, nonseptate.

6. Thallus not umbilicate, smaller, squamulose.

4. Dermatocarpon

7. Squamules very small; perithecia rare; spores hyaline or brownish, septate.

3. Normandina

7. Squamules not very small; perithecia not rare; spores hyaline, nonseptate.

4. Dermatocarpon

5. Spores borne in an apothecium.

8. Thallus squamulose.

9. Apothecia borne in a stroma; disk black; spores 1-septate.

8. Arthonia

9. Apothecia not borne in a stroma.

10. Usually a green algal component, very rarely a blue-green.

11. Thalline margin conspicuous, permanent.

12. Spores small to very small, asci often 100 -spored.

13. Thallus with an upper cortex, usually marginally lobed.

44. Acarospora

13. Thallus without an upper cortex, usually not marginally lobed.

12. Spores neither very large nor very small.

51. Candelariella

14. Spores hyaline.

15. Spores nonseptate. 
16. Always growing on moss, sometimes almost covered by it.

16. Rarely 24. Psoroma

16. Rarely growing on moss, never covered by it.... 46 . Lecanora

15. Spores polar-bilocular; epithecium crimson, dull red, or purple with $\mathrm{KOH}$.

65. Caloplaca

14. Spores brown or black, polar-bilocular.

68. Rinodina

11. Thalline margin absent, or soon disappearing.

17. Spores nonseptate, medium sized.

31. Lecidea

17. Spores 1-many transversely septate, or muriform.

18. Spores without a gelatinous halo.

19. Spores hyaline, never muriform

35. Toninia

19. Spores brown or black, rarely muriform.

18. Spores with a gelatinous halo, usually brown, muriform.

67. Buellia

37. Rhizocarpon

10. Always a blue-green algal component.

20. Spores nonseptate.

21. Thalline margin conspicuous, concolorous with the thallus.

21. Thalline margin absent.

22. Pannaria

20. Spores 1-septate, thalline margin absent.

21. Parmeliella

8. Thallus foliose.

23. Massalongia

22. Attached to the substratum by a single umbilicus or by several cords.

42. Umbilicaria

22. Not attached to the substratum by a single umbilicus or by several cords.

23. Apothecia originate on the lower surface of the thallus.

24. Apothecia remain on the margin of the lower surface...22. Nephroma

24. Apothecia appear to grow on the margin of the upper surface because of the twisting of the lobes of the thallus................56. Nephromopsis

23. Apothecia originate on the upper surface of the thallus.

25. A pothecia marginal or submarginal.

26. Growing in swift mountain brooks, a blue-green algal component.

26. Not growing in swift mountain brooks.

25. Hydrothyria

27. Thallus red, purple, or violet, with $\mathrm{KOH}$.

27. Thallus not changing color with $\mathrm{KOH}$.

28. Lower cortex interrupted by cyphellae.

28. Lower cortex not interrupted by cyphellae.

66. Xanthoria

29. Lower cortex interrupted by naked, inflated, flat, or sunken spots. .................................................................26. Lobaria

29. Lower cortex not interrupted by naked, inflated, flat, or sunken spots.

30. Rhizoids abundant on the lower surface of the thallus, thalline margin of apothecium absent.

30. Peltigera

30. Rhizoids rather scarce on the lower surface of the thallus, thalline margin of apothecium present.

25. Apothecia scattered over the surface of the thallus.

55. Cetraria

31. Lower cortex interrupted by cyphellae.

31. Lower cortex not interrupted by cyphellae.

27. Sticta

32. Lower cortex interrupted by naked, light colored, inflated, flat, or sunken spots.

26. Lobaria

32. Lower cortex not interrupted by naked, light colored, inflated, flat, or sunken spots.

33. Spores numerous; asci 16-many-spored; spores nonseptate or rarely 1-septate. 52. Candelaria

33. Spores not numerous; asci normally 8-spored.

34. Spores brown. 
35. Thalline margin absent, spores 1-septate.

28. Solorina

35. Thalline margin present, spores 1 -septate or muriform.......

69. Physcia

34. Spores hyaline.

36. Spores polar-bilocular; thallus red, purple, or violet with $\mathrm{KOH}$. 66. Xanthoria

36. Spores nonseptate.

37. Upper cortex poorly developed, thallus always small, often covered with soredia or breaking up into powdery masses.

53. Parmeliopsis

37. Upper cortex well developed, thallus various in size..

54. Parmelia

5. Perithecia and apothecia both absent.

38. Thallus growing on rocks immersed in water.

39. Always found growing in mountain streams; algal component blue-green.

25. Hydrothyria

39. Not always in mountain streams; algal component green.

4. Dermatocarpon

38. Thallus not growing on rocks immersed in water.

40. Thailus squamulose.

41. Thallus red or purple with $\mathrm{KOH}$

65. Caloplaca

41. Thallus not red or purple with $\mathrm{KOH}$.

42. Always a blue-green algal component.

42. Always a green algal component.

43. Attached to substrate by hyphal rhizoids; no lower cortex.

21. Parmeliella

43. Attached to the substrate by rhizoids or an umbilicus.

51. Candelariella

44. Thallus very small, thin and dainty, upturned edges sorediate......

3. Normandina

44. Thallus larger, thicker and coarser; edges not upturned, not sorediate.

4. Dermatocarpon

40. Thallus foliose.

45. Thallus attached to the substrate by a single umbilicus or several cords.

46. Thallus thin and papery when dry......

42. Unbilicaria

46. Thallus never so thin and papery when dry......

4. Dermatocarpon

45. Thallus not attached to the substrate by a single umbilicus or several cords.

47. Thallus red or purple with $\mathrm{KOH}$.

48. Thallus with both upper and lower cortices, with rhizoids or a definite holdfast. 65. Xanthoria

48. Thallus not as above, usually yellow or orange.

65. Caloplaca

47. Thallus not red or yellow with $\mathrm{KOH}$.

49. Small, rather delicate lichens.

50. Medulla cottony.

69. Physcia

50. Medulla not cottony.

51. Thallus with both upper and lower cortices; spermatia lateral.

52. Candelaria

51. Thallus with only a lower cortex

53. Parmeliopsis

49. Larger, coarser lichens.

52. Lower surface of thallus tomentose or villous, interrupted by cyphellae or naked, light-colored, inflated spots.

53. Lower surface interrupted by cyphellae.

27. Sticta

53. Lower surface interrupted by naked, light-colored, inflated spots.

26. Lobaria

52. Lower surface of thallus not as above.

54. Thallus upright, ascending, rhizoids usually lacking on lower 
surface.

55. Cetraria

54. Thallus flat, prostrate; rhizoids usually abundant, rarely lacking on the lower surface.

54. Parmelia

\section{FRUTICOSE}

1. Podetia present (thallus usually twofold, consisting of a primary crustose, squamulose, or foliose thallus that often disappears, and a secondary thallus consisting of erect podetia).

2. Podetia short, often poorly developed, usually simple.

3. Apothecia black.

39. Pilophoron

3. Apothecia light-colored.

38. Baeomyces

2. Podetia longer, well developed, usually branched.

4. Podetia solid, cephalodia common.

41. Stereocaulon

4. Podetia usually hollow, simple or branched.

5. Podetia always white or ashy, simple or sparingly branched, apothecia unknown.

63. Thamnolia

5. Podetia not always white or ashy, simple or abundantly branched, apothecia usually present, disk brown, reddish brown, or scarlet.

40. Cladonia

1. Podetia absent.

6. Apothecia stalked.

7. Stalk long, well developed.

5. Calicium

7. Stalk short, sometimes lacking.

8. Thallus greenish, resembling a layer of paint; disk pale flesh color ( $R$ ) or rosy pink.

48. Icmadophila

8. Thallus grayish, disk dull black.

6. Cyphelium

6. Apothecia not stalked.

9. Thallus gelatinous when moist, brittle when dry; always a blue-green algal component.

20. Leptogium

9. Thallus not gelatinous when moist.

10. Thallus dark green, dark brown, or black.

11. Thallus consisting of small, fine hair or thread-like masses.

12. Blue-green algal component; fungal hyphae entirely within the algal filament.

17. Thermutis

12. Green algal component; fungal hyphae not within the algal filament.

54. Parmelia

11. Thallus consisting of large, coarse hair or string-like masses, erect, procumbent, or pendulous.

59. Alectoria

11. Thallus shrub-like, membranaceous, erect, depressed, or procumbent.

13. Lower cortex rarely developed, thalline margin absent........31. Lccidea

13. Lower cortex well developed, thalline margin present.

14. Thallus dark brown or black on both surfaces, apothecia scattered on upper surface.............................................................54. Parmelia

14. Thallus dark brown, greenish black, or black on upper surface, lighter below, apothecia marginal.

55. Cetraria

13. Cortex extending all the way around the thallus, thalline margin present.

10. Thallus light brown, brown, or yellow.

60. Cornicularia

15. Medulla cottony.

16. Apothecia originate on the lower surface of the thallus, but by the turning of the lobes of the thallus appear to grow on the margin of the upper surface.....................................................56. Nephromopsis

16. Apothecia originate on the margin of the upper surface of the thallus.

15. Medulla stiff or cobweb-like, thallus always some shade of yellow.

55. Cetraria

58. Letharia 
15. Medulla solid; apothecia terminal, within swollen globose receptacles.

7. Sphaerophorus

10. Thallus greenish yellow, straw color, grayish green, gray, or white.

17. Thallus consisting of hair-like or string-like masses.

18. Medulla with a strong central medullary cord.

62. Usnea

18. Medulla hollow in the center.

59. Alectoria

17. Thallus not consisting of hair-like or string-like masses, but wider, membranaceous.

19. Thallus soft and flaccid, medulla cottony......

57. Evernia

19. Thallus rather stiff, cartilaginous, medulla soft and cottony or cobweblike.

61. Ramalina

17. Thallus shrub-like, terete, brittle, apothecia terminal within swollen globose receptacles.

7. Sphacrophorus

\section{VERRUCARIACEAE}

Thallus crustose, entirely superficial or partly developed within the substratum, no differentiation into layers. Perithecia simple, erect, more or less immersed, with apical pore or ostiole protruding; asci 1-8-spored or many spored, spores hyaline or rarely brownish, nonseptate, 1-3-septate, or muriform.

Alga Protococcus or Palmella.

A. Spores simple, hyaline; dark hypothallus evident.

1. Verrucaria A. Spores muriform, brown, hypothallus not often seen.......2. Staurothele

1. Verrucaria Scop. Intro. Hist. Nat. 61. 1777.

Thallus crustose, usually superficial, rarely within the substratum, sometimes areolate, dark hypothallus often visible. Perithecia partly immersed in the thallus, superficial part globose or hemispherical, opening by a pore at the apex; paraphyses indistinct, soon gelatinizing; asci 8-spored; spores hyaline, nonseptate.

Alga Protococcus.

A. On rocks along the seacoast, spores always less than $20 \mu$ in length.

1. V. maura

A. On rocks in dry places, spores rarely less than $20 \mu$ in length.

2. V. nigrescens

1. Verrucaria maura Wahl. in Ach. Meth. Lich. 19. 1803.

Thallus dark mouse gray ( $R$ ), reddish black, or black, thin or thick, smooth to rough, or cracking into small areoles. Perithecia minute to small, 0.3-0.5 mm. across, partly immersed in the thallus, superficial part black, globose or hemispherical; spores hyaline, ellipsoid, nonseptate, 9.9-16.5 x 4.9-8.0 $\mu$.

On rocks near the sea: San Juan Co. : Turn Island, 1928, 1987.

2. Verrucaria nigrescens Pers. Ann. Bot. Usteri $14: 36.1795$.

Verrucaria elaeochroa Tuck. Pro. Am. Arts and Sci. I :279. 1848. 
Thallus brownish or dull black, rather thick, in small mats or wide spread, smooth to rough, becoming cracked, areolate. Perithecia minute, 0.1-0.3 $\mathrm{mm}$. across, partly immersed in the thallus, hemispherical; spores hyaline, ellipsoid, nonseptate, $15.0-20.0 \times 7.0-8.0 \mu$.

On rock: Whatcom Co.: Sumas Mountain, Herre (1917). Washington Territory, San Juan Island, Suksdorf $285 b$ (F).

2. Staurothele Norm. Nyt. Mag. Nat. $7: 240.1853$.

Thallus crustose, smooth to granulose or warty, thick or sometimes thin, areolate, usually partly within the substratum, hypothallus rarely visible. Perithecia minute to small, immersed in the thallus, ostiole either depressed or elevated; paraphyses soon dissolving; asci 1-8-spored; spores large, hyaline or brown, muriform.

Alga Protococcus.

A. Perithecia 0.1-0.3 mm. across, ostiole depressed.

1. S. clopima

A. Perithecia not less than $0.3 \mathrm{~mm}$. across, ostiole, elevated......2. S. lithina

1. Staurothele clopima (Wahl.) Th. Fr. Nov. Act. Reg. Soc. Sci. 111. 3 :363. 1861. Verrucaria clopima Wahl. in Ach. Meth. Lich. 19. 1803. Staurothele umbrina var. clopima (Wahl.) Tuck. Gen. 258. 1872.

Thallus light or dark brown, thick, warty, tumid, rounded areoles continuous or broken up into small patches. Perithecia minute, $0.1-0.3 \mathrm{~mm}$. across, immersed in the thallus; ostiole depressed, appearing as a tiny hole in the surface; asci 1 or 2-spored; spores brown, oblong, muriform, 11.0$30.0 \times 11.0-26.4 \mu$.

On rock: Chelan Co.: Beaver Summit, 2800 ft., 1931, 1342. Okanogan Co. : Twisp Butte, near Twisp, $1700 \mathrm{ft} ., 1931,1465$.

2. Staurothele lithina (Ach.) Zahlbr. Cat. Lich. Univ. 1 :172. 1922. Verrucaria lithina Ach. Meth. Lich. 18. 1803. Staurothele umbrina (Ach.) Tuck. Gen. 258. 1872.

Thallus dark brown or brownish black, rather thin, smooth, granulose, or areolate. Perithecia minute, 0.3-0.5 mm. across, immersed in elevated spots in the thallus, with ostioles projecting; asci 1 or 2 -spored; spores brown, oblong, muriform, 33.0-49.6 x 14.0-16.5 $\mu$.

On rock: Okanogan Co.: Twisp State Trail, 4000 ft., 1931, 1297. Yakima Co.: Lodge Pole Pine Camp, 3500 ft., 1940, 3309. Washington Territory, Suksdorf 269 (F).

Herre (1943) reports Staurothele glacialis Herre sp. nov. on lava rock, Whatcom Co. : Mt. Baker, $7000 \mathrm{ft}$.

\section{DERMATOCARPACEAE}

Thallus foliose, squamulose, or subcrustose, corticate on one surface or on both, algal and medullary layers well developed, attached to the substratum either by an umbilicus or rhizoids. Perithecia minute to small, im- 
mersed in the thallus, only the ostiole showing on the surface as a small pore; asci 1-16-spored; spores hyaline, brown, or blackish, nonseptate, multiseptate, or muriform.

\section{Alga Protococcus.}

A. Thallus attached to the substratum by rhizoids, spores 5-7-septate.

3. Normandina

A. Thallus usually attached to the substratum by an umbilicus, if squamulose not umbilicate, spores nonseptate.

4. Dermatocarpon

3. Normandina Nyl. Mem. Soc. Sci. Nat. Cherbourg, $3: 191.1855$.

Thallus small, squamulose orbicular, upper cortex poorly developed, definite algal and medullary layers, lower cortex well developed, attached to the substratum by rhizoids. Perithecia rare; paraphyses lacking; asci 6-S-spored; spores hyaline to brownish, 5-7-septate or rarely many-septate. A monotypic genus.

1. Normandina pulchella (Borr.) Nyl. Ann. Sci. Bot. IV. 15:382. 1861. Verrucaria pulchella Borr. in Sowerby, Eng. Bot. Suppl. 1 :2602. 1831. Normandina jungermanniae Nyl.

Thallus glaucous-gray ( $R$ ), light olive-gray ( $R$ ), consisting of small, thin, dainty squamules, faintly pruinose, often sorediate, margin raised, thickened, and slightly reflexed, below white or pale brown. Perithecia rare, immersed in raised areas, only the small black ostiole showing above the surface; asci 8 -spored; spores hyaline, brown when mature, cylindrical, 6-7-septate, $26.0-40.0 \times 6.0-8.0 \mu$.

On mosses growing over the bark of trees or on soil: Pacific Co.: Loomis Lake, 1931, 734, 428. Washington Territory, Suksdorf 89 (T). Determined as Normandina jungermanniae Nyl. by Tuckerman.

\section{Dermatocarpon Eschw. Syst. Lich. 21. 1824.}

Thallus foliose, large and spreading, umbilicate or reduced to appressed and adnate squamules, upper cortex thin, lower thicker, algal and medullary layers well developed; attached to the substratum by an umbilicus or rhizoids. Perithecia minute or small, immersed in the substratum, wall dimidiate; paraphyses gelatinizing; asci 8-spored or rarely 16-spored; spores hyaline, nonseptate.

A. Thallus foliose, umbilicate.

$\mathrm{B}$. Thallus small or medium sized; below not veined; growing in water or moist situations.

C. Thallus thick and flaccid; below brown or black.

C. Thallus thinner, firm and hard; below always black.

1. D. aquaticum

5. D. Mühlenbergii

B. Thallus large; below faintly veined; growing in dry situations.

D. Thallus lobes much imbricated...4. D. miniatum var. complicatum 
D. Thallus lobes rarely imbricated.

3. D. miniatum

A. Thallus squamulose, not umbilicate.

E. Squamules divided into many small lobes, often with acute ascending tips; below smooth.

6. D. polyphyllum

E. Squamules entire; below bearing rhizoids.................. 2. D. hepaticum

1. Dermatocarpon aquaticum (Weis.) Zahlbr. Ann. Nat. Hofmus. Wien. 16:81. 1901. Lichen aquaticus Weis. Pl. Crypt-F1. Goett. 77. 1770. Endocarpon fluviatile (Web.) DC. Dermatocarpon fluviatile (IVeb.) Th. Fr.

Thallus grayish-brown, brown, or Isabella color ( $R$ ), foliose, small to medium, thick, coriaceous, smooth or very minutely granular-pruinose, many lobed, crowded, margins entire or somewhat sinuate; below brown or black, attached to the substratum by an umbilicus. Perithecia appearing on the surface as very small dark spots; asci 8-spored; spores hyaline, ellipsoid, nonseptate, $10.5-16.0 \times 6.0-9.5 \mu$.

On rocks usually submerged in water: San Juan Co.: Brown Island, 1940, 3257. Jefferson Co.: Queets River Valley, in streamlet, 5000 ft., 1907, Frye 13, (F). Determined as Endocarpon fluviatile DC.

2. Dermatocarpon hepaticum (Ach.) Th. Fr. Nov. Act. Reg. Soc. Sci. Upsal. 111, 3 :355. 1861. Endocarpon hepaticum Ach. in Kgl. VetenskAkad. Nya. Handl. 156. 1809.

Thallus reddish brown, or dusky brown ( $R$ ), squamulose; squamules small or medium sized, circular or irregular in shape, flat, margin often blackish, entire or crenate, somewhat imbricated; below almost black; closely attached to the substratum by fine rhizoids. Perithecia numerous, appearing on the surface as tiny dark spots; asci 8 -spored; spores hyaline, ovoid, nonseptate. 9.0-14.0 × 5.0-8.0 $\mu$.

On soil: Cascade Mountains, 1883, Brandegee 17 (T).

3. Dermatocarpon miniatum (L) Mann, Lich. Bohem. observ. Dispos. 66. 1825. Lichen miniatus Linn. Sp. P1. 1149. 1753. Endocarpon miniatum (L.) Gart.

Thallus grayish olive ( $R$ ), deep grayish olive (R), or various shades of brown, foliose, rather large and spreading, coriaceous, lobes rounded plicate, surface smooth or minutely granular-pruinose; below usually darker fuscous-black ( $R)$, blackish brown or light brownish-olive $(R)$, smooth to minutely granular-pruinose, sometimes slightly veined; loosely attached to the substratum by an umbilicus. Perithecia minute, numerous; asci 8spored; spores hyaline, ovoid to ellipsoid, nonseptate, 6.6-16.5 x 3.9-6.6 $\mu$.

On rock: Yakima Co.: Deadhorse Hill, 4000 ft., 1931, 989. Klickitat Co.: Goldendale, 2000 ft., 1931, 1695. Okanogan Co.: butte near Twisp, 1931, 1460. Ferry Co. : between Curlew and Danville, 1773 ft., 1931, 1414. Whitman Co.: Pullman, 2550 ft., 1931, 1578. San Juan Co.: Waldron 
Island, 1905, Frye (W). Mason Co. : Mt. Elinor 1912, Foster 2099 (F). Ferry Co. : Republic, 1912, Foster 2303 (F).

4. Dermatocarpon miniatum var. complicatum (Lightf.) Th. Fr. Nov. Act. Soc. Ups. 111. 3 :353. 1861. Lichen miniatus var. complicatus Lightf. Fl. Scot. 858. 1777.

Thallus small, deeply divided, often divided almost to the umbilicus, compacted-imbricated, raised from the substratum; otherwise like the species.

On rock: Yakima Co.: American River Camp, 2800 ft., 1931, 990. Okanogan Co.: Twisp Trail, 4000 ft., 1931, 1299. Ferry Co.: between Curlew and Danville, 1644 ft., 1931, 1414a. Whitman Co.: Pullman, 2550 ft., 1931, 1578a. San Juan Co.: Waldron Island 1905, Frye (F). Ferry Co. : Republic 1913, Foster 2303a (F).

5. Dermatocarpon Mühlenbergii (Ach.) Müll. Arg. in Engler Bot. Jahrb. 6:377. 1855. Endocarpon Mühlenbergii Ach. Syn. Lich. 101, 1814. Endocarpon miniatum var. Mühlenbergii Calkins.

Thallus light drab $(\mathrm{R})$ or brown, foliose, small or medium sized, circular, often with circular lobes, coriaceous, smooth to very minutely granularpruinose, margin sinuate, involute; below black, smooth to very minutely granular-pruinose; attached to the substratum by an umbilicus. Perithecia appearing on the surface as tiny dark spots; asci 8-spored; spores hyaline, ovoid to ellipsoid, $13.2-16.5 \times 5-6.6 \mu$.

On rock usually submerged in water: Pierce Co. : Mt. Rainier, Berkeley Park, 6000 ft., 1931, 629, 630, 631. Walla Walla Co.: Wallula, 1906, Foster 290 (F). Ellensburg, Roell \& Dieck 63 (F).

6. Dermatocarpon polyphyllum (Wurf.) Dalla Torre \& Sarnth. Die Flecht. Tirol, 504. 1902. Lichen polyphyllus Wulf. in Schrift. Gesellsch. Naturf. Freund. Berlin 8:142. 1787.

Thallus Isabella color $(\mathrm{R})$ or tawny-olive $(\mathrm{R})$, small to medium sized, foliose, thick, deeply divided into small revulute lobes, margins rounded crenate with acute ascending tips, or squamulose; squamules imbricated, very compact; below blackish brown or black, smooth or slightly veined. Perithecia minute; asci 8-spored; spores hyaline, ellipsoid, nonseptate, 9.9-13.2 $\times$ 4.9-5.0 $\mu$.

On rock: Yakima Co.: American River Camp, 2800 ft., 1931, 990a.

\section{CALICIACEAE}

Thallus crustose, partly within the substratum, well developed or sometimes disappearing entirely, little or no differentiation into layers. Apothecia top-shaped or globose on a slender stipe, which is usually simple, sometimes branched with an apothecia on each branch; proper margin usually present, thalline lacking; asci 8-spored; spores hyaline or dark-colored, nonseptate or 1-septate.

Alga Protococcus, Cystococcus, or Stichococcus. 
5. Calicium Pers. Neue Ann. Bot. $1: 20.1794$.

Thallus smooth to granular or pulverulent, sometimes evanescent. Apothecia blackish to black, often slightly pruinose; stipes well developed; proper margin present; hypothecium brown; paraphyses slender, threadlike; asci 8-spored; spores pale brown, 1-septate, slightly constricted at the septum.

\section{Alga Cystococcus.}

1. Calicium hyperellum Ach., Meth. Lich. 93. 1803. Lichen hyperellus Ach. Lich. Suec. Prodro. 85, 1798.

Thallus clear dull green yellow ( $R$ ), granular or pulverulent, well developed and effuse, or thin and scattered. Apothecia brown to black, globose, lentiform or top-shaped, 0.1-0.2 mm. across, supported by a firm elongated stalk; disk convex to flat; proper margin brownish black; spores pale brown, oblong-ellipsoid, 1-septate, 6.9-13.2 x 4.0-6.6 $\mu$.

On bark and decorticated wood: San Juan Co.: Brown Island, 1940, 3255. Yakima Co.: Rattlesnake Canyon, 2000 ft., 1931, 1541. Whatcom Co.: 1913, Herre 405 (F). West Klickitat Co.: Columbia River, 1882, Herb. of C. B. Faxon (F). Ferry Co.: Republic 1912, Foster 2301 (F).

\section{CYPHELIACEAE}

Thallus uniformly crustose to radiately lobed at the margin, sometimes partly within the substratum, often rudimentary, with no differentiation into layers. Apothecia spherical to globose, adnate to sessile, both proper and thalline margins present or with only a thalline margin ; asci 8-to-manyspored; spores light-colored or brown, non-to-3-septate.

Alga Protococcus or Trentepohlia.

\section{Cyphelium Ach. Vet. Akad. Handl. 263. 1815.}

Thallus smooth to granulous, warty or areolate, margin sometimes lobed. Apothecia immersed in the thallus, or wholly or partly superficial; thalline margin present and sometimes a proper one within the substratum; stalk very short or wanting; hypothecium brown; paraphyses thread-like and sparse; asci 8-spored; spores brown, normally 1-septate, rarely nonseptate or 3-septate, usually constricted at the septa.

Alga Protococcus.

1. Cyphelium inquinans (J. E. Smith) Trevis. Flora 45:4. 1862. Lichen inquinans Smith in Sowerby Eng. Bot. 12: tab. 801. 1801.

Thallus gray or grayish-olive (R), granulose or warty, well developed or thin and scattered. Apothecia usually elevated, semi-globose; stalk very short, scattered, 0.5-1.2 $\mathrm{mm}$. in diameter; disk dull black, sometimes faintly white pruinose, flat; proper margin brown, thin, surrounded by a darker brown, white pruinose thalline margin, sometimes disappearing with age; spores brown, oblong, 1 -septate, $13.2-20.0 \times 7.3-13 \mu$. 
On trees, old wood, and rarely on rocks: Yakima Co.: Rattlesnake Canyon, 2000 ft., 1931, 1542. San Juan Co.: San Juan Island, 1906, Fink 60 (F). Clallam Co. : Pt. Angeles, 1911, Foster (F). Whatcom Co.: 1916, Herre (W). Island Co. : Whidbey Island, Langley, 1920, Grant 3032 (F). Thurston Co.: Gate, 1912, Foster 1979 (F). Yakima Co.: Mt. Adams, 1881, Pringle (F). Ferry Co. : Republic, 1912, Foster 2300 (F). Washington Territory, Suksdorf (F).

This species can easily be determined by the short-stalked, semi-globose apothecia and the brown, oblong, 1-septate spores.

\section{SPHAEROPHORACEAE}

Thallus foliose or fruticose, with some differentiation into cortex, algal layer and medulla, cortex usually equally developed on all surfaces. Apothecia borne laterally or terminally on the lobes or branches of the thallus, adnate to sessile surrounded by a thalline margin; asci 8-spored; spores hyaline, violet-brown to brown, nonseptate or 1 -septate.

Alga Protococcus.

\section{Sphaerophorus Pers. Neue Ann. der Bot. 1 :23. 1794.}

Thallus fruticose, erect, cortex cartilaginous, medulla forming rather a solid cylinder, surrounded by the algal layer. Apothecia borne on the ends of the branches, within globose, swollen thalline receptacles, opening irregular near the top; hypothecium hyaline; paraphyses delicate; asci 8-spored; spores hyaline with a thin violet-black, dust-like epispore, nonseptate.

1. Sphaerophorus globosus (Huds.) Wainio, in Result. Voyage S. Y. Belgica, Bot. 35. 1903. Lichen globosus Huds. Fl. Angelica 460. 1762. Sphaerophorus globiferus (L.) DC.

Thallus silvery gray, reddish-brown, or pale olive-buff $(R)$, shrub-like loosely branched, terete, smooth and shiny, brittle and breaking easily, with numerous clusters of small coralloid branchlets. Apothecia terminal, 0.5-2.0 $\mathrm{mm}$. in diameter, within thalline receptacle; disk black, flat; spores hyaline with a thin violet-black epispore, spherical, 6.6-16.0 $\mu$ in diameter.

On soil, rocks, trees, and fallen logs: Pacific Co.: Loomis Lake, 1931, 775. Kitsap Co. : Hidden Ranch near Bremerton, 213 ft., 1931, 1737. Whatcom Co.: Ruth Mountain, 5500 ft., 1928, Sunquist 1843 (W). Pierce Co.: Enumclaw, 442 ft., 1931, 687 ; Mt. Rainier, trail to Mowich Lake, $3500 \mathrm{ft}$., 1940, 3065. King-Kittitas Co.: Snoqualmie Pass, 3010 ft., 1931, 812. Clallam Co.: Olympic Mountains, Elwha River Valley, 2000 ft., 1907, Frye 8 (W). Wahkiakum Co.: Cathlamet, 1907, Foster 508 (W). Thurston Co.: Gate, 1912, Foster 2017 (W). Skamania Co. : Little Soda Springs Camp Grounds, 1933, L. D. Lawrence (W).

Collected only west of the Cascade Mountains, where it is widely distributed, common on tree trunks. It is easily recognized by the erect, terete branches, bearing numerous branchlets. Medulla turns blue with IKI. 


\section{ARTHONIACEAE}

Thallus crustose, thin, often beneath the substratum or lacking entirely, no differentiation into layers. Apothecia circular to linear, sometimes branched, single or clustered, in a stroma; proper margin absent or very poorly developed, thalline margin absent; asci 1-8-spored; spores hyaline or brown, 1-many-septate or muriform.

Alga Palmella, Trentepohlia, or Phyllactidium.

8. Arthonia Ach. Neu. Jour. fur Bot. 1: Part 3. 3. 1806.

Thallus crustose to rarely areolate, or wanting, thin to rather thick, sometimes developed within the substratum. Apothecia circular, elliptical, or star-shaped, immersed to adnate; both margins absent; hypothecium usually brownish; paraphyses much branched; asci usually 8-spored, sometimes 4-6, 6-8, or rarely 1-2, pyriform; spores hyaline or rarely brown, 1-many-septate, the cells often of unequal size.

Alga Trentepohlia.

A. Thallus crustose

B. Disk white pruinose.

4. A. impolita

B. Disk not white pruinose, usually black.

C. Apothecia irregular in shape, often branched.

D. Spores 3-septate.

5. A, radiata

D. Spores 5-septate.

2. A. excedens

C. Apothecia elongated, not branched.

1. A. complanata

A. Thallus squamulose.

3. A. glebosa

1. Arthonia complanata Fee, Essai Crypt. Ecorc. Officin. 54. 1824.

Thallus grayish white or grayish green, superficial, thin, smooth to rough and cracked. Apothecia 0.2-0.6 mm. wide, $1.3 \mathrm{~mm}$. long, immersed to adnate; disk black, flat to convex; asci 8 -spored; spores hyaline to brownish, ovoid or ovoid-ellipsoid with one or both end cells enlarged, 4-6-septate,

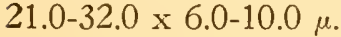

On bark: Grays Harbor Co.: Westport, 1908, Foster 670 (F). San Juan Co. : San Juan Island, Friday Harbor, Fink (1935).

2. Arthonia excedens Nyl., Act. Soc. Sci. Fenn. 7 :484. 1863.

Thallus grayish white to gray, superficial, thin to very thin. Apothecia dark 0.5-0.8 mm. wide, $2.0 \mathrm{~mm}$. long, circular to slightly irregular somewhat branched, partly immersed to adnate; disk black, rarely dark brown, when moist, reddish, flat to convex; asci 4-6-spored; spores hyaline or tinged with brown, ovoid-ellipsoid, 5-septate, 26.4-34.9 x 7.3-11.4 $\mu$.

On bark: Pacific Co.: Loomis Lake, 1931, 735, 1934, 2442; Chinook, 1940, 2807. Wahkiakum Co.: Cathlamet, 1940, 2918. Grays Harbor Co.: Westport, 1908, Foster 718 (F). Thurston Co.: Gate, 1912, Foster (F).

3. Arthonia glebosa Tuck. Gen. Lich. 221, 1872. 
Thallus dusky or a smoky drab color, thick, tumid, consisting of elevated squamules. Apothecia rounded 2.0-5.0 mm. in diameter, often crowded and confluent, immersed to adnate; disk black, convex, hypothecium brownishblack; asci 8-spored; spores pale brown to hyaline, ovoid or oblong-ovoid, 1-septate constricted in the middle, $10.0-17.0 \times 4.5-6.0 \mu$.

On moss, rock, or on a thin layer of soil over rock: Washington Territory, Suksdorf $32(\mathrm{~F})$.

4. Arthonia impolita (Ehrh.) Borr. in Hook. \& Sowerb. Eng. Bot. 1: tab. 2692, f. 1, 1831. Lichen impolita Ehrh., Pl. Crypt. Exs. no. 274. 1793.

Thallus grayish white or pale olive-buff $(R)$, rather thick, smooth or rough, and chinky, crumbling. Apothecia $0.2-1.0 \mathrm{~mm}$. in diameter, circular to elliptical, often clustered, partly immersed to adnate; disk brown or black, white pruinose, flat; asci 8-spored; spores hyaline, ovoid-ellipsoid, 3-5-septate, 13.2-16.5 x 4.9-6.0 $\mu$.

On bark and old wood: San Juan Co.: Turn Island, 1928, 1988; San Juan Island, 1906, Fink 1919.

5. Arthonia radiata (Pers.) Ach., Kgl. Vet-Akad. Nya Handl. 131, 1808. Opegrapha radiata Pers. Ann. Bot. Usteri, 7 :29. 1794.

Thallus whitish, grayish green, sometimes lacking, occasionally bordered by a black line, smooth to slightly rough. Apothecia $0.2-0.9 \mathrm{~mm}$. in diameter, irregularly shaped, often stellate, slightly immersed to adnate; disk black, flat; asci 8 -spored; spores hyaline, ovoid-ellipsoid, 3-septate, 10.5-19.0 × 4.0-5.9 $\mu$.

On bark: Clallam Co.: near Port Angeles, 1940, 2923. Washington Territory, 1883, Suksdorf 255 (F). 


\section{GRAPHIDACEAE}

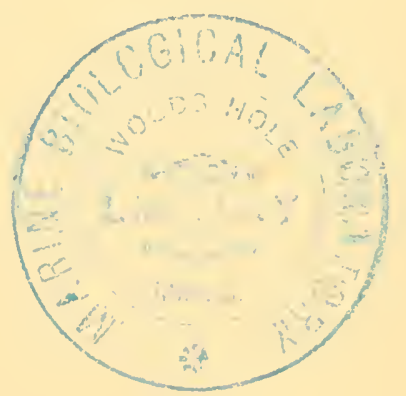

Thallus crustose, superficial or entirely within the substratum, little or no differentiation into layers. Apothecia lirellae form, black, elongated to circular, single or clustered, not in a stroma; proper margin usually well developed, thalline margin present or absent; asci 4-8-spored; spores hyaline, or brown, nonseptate to multiseptate.

Alga Palmella or Trentepohlia.

A. Spores simple; thallus entirely within the substratum........9. Xylographa A. Spores septate; thallus usually evident on the surface of the substratum.

B. Paraphyses branched and interwoven; apothecia immersed and then erumpent.

11. Graphis

B. Paraphyses unbranched and distinct; apothecia superficial.

10. Opegrapha

9. Xylographa Fries, FI. Scan. 344. 1835.

Thallus crustose or entirely within the substratum. Apothecia black or brown, erumpent, circular to linear, clustered or in parallel rows; proper margin thin and dark colored, thalline margin absent; hypothecium hyaline to slightly colored; paraphyses several-celled, rarely branched; asci 8spored; spores hyaline, nonseptate.

Alga Palmella.

A. Apothecia in more or less parallel lines, elongated, reaching $3.0 \mathrm{~mm}$. in length.

1. X. abietina

A. Apothecia irregularly clustered, circular to oblong not exceeding 0.6 $\mathrm{mm}$. in length.

2. $X$. hians

1. Xylographa abietina (Pers.) Zahlbr. Cat. Lich. Univ. 2:151. 1924. Hysterium abietinum Pers. Obser. Mycol. 1 :1796. Xylographa parallela (Ach.) Behlen \& Desberg.

Thallus usually entirely within the substratum, rarely showing on the surface as a grayish coloration. Apothecia 0.5-3.0 x 0.2-0.5 mm. long, and narrow, innate, finally erumpent, more or less in parallel lines; disk black, varying from closed to open, concave to flat; proper margin black; spores hyaline, ellipsoid, nonseptate, $11.0-16.5 \times 5.0-6.6 \mu$. 
On old wood: Yakima Co. : Deadhorse Hill, 4000 ft., 1931, 971. Washington Territory, 1882, Suksdorf 173 (T).

2. Xylographa hians Tuck. Syn. N. Am. Lich. 2:113. 1888.

Thallus usually within the substratum, rarely showing on the surface, as a grayish coloration. Apothecia 0.1-0.2 $\times 0.2-0.6 \mathrm{~mm}$. in diameter, circular to oblong, adnate, clustered; disk brown, concave to flat; proper margin thin, concolorous with the disk; spores hyaline, ovoid, nonseptate, 9.9-13.2 x 4.9-6.6 $\mu$.

On old wood: Grays Harbor Co.: Aberdeen, 1909, Foster (F). Washington Territory, Suksdorf 233 (T), Type specimen.

\section{Opegrapha Humb. Fl. Frib. Specim. 57. 1793.}

Thallus crustose, thin to slightly chinky or powdery, rarely disappearing entirely within the substratum, no differentiation into layers; apothecia black, carbonaceous; lirellae, linear to almost circular, immersed to adnate; proper margin well developed, thalline margin usually absent; hypothecium yellowish, brown, or black; paraphyses branched and interwoven; asci cylindrical, thin walled, 8-spored; spores hyaline, multiseptate.

Alga Trentepohlia.

A. Spores constantly 3 -septate, surrounded by a thick gelatinous halo.

4. O. protuberans

A. Spores 3-7-septate, not surrounded by a gelatinous halo.

B. Spores constantly 3-septate............................................ O. Otra

B. Spores not constantly 3-septate.

C. Spores always hyaline.

2. O. diaphora

C. Spores always hyaline, brown at maturity.

D. Thallus smooth, apothecia subsessile.

3. O. lichenoides

D. Thallus rough, apothecia immersed to adnate.

5. O. varia

1. Opegrapha atra Pers., Neu. Ann. Bot. Usteri 7 :30. 1794.

Thallus white, thin, smooth, sometimes disappearing within the substratum. Apothecia spindle shaped, 0.2-3.0 × 0.2-0.4 mm., adnate, straight or rarely curved; disk black, usually closed, rarely narrowly opened; proper margin black; hypothecium brownish black; spores hyaline, ellipsoid larger at one end, 3-septate, 15.2-19.0 x 4.0-5.7 $\mu$.

On bark and old wood: Pierce Co.: Mt. Rainier White River Camp, 3900 ft., 1940, 3106a. Clallam Co.: Dungeness, 1913, Foster 2532 (F).

This species can be distinguished by the rather long narrow apothecia.

2. Opegrapha diaphora Ach. Meth. Lich. 19. 1803. Lichen diaphorus Ach. Lich. Suec. Prodr. 20. 1798. Opegrapha varia var. diaphora Fries.

Thallus gray to greenish gray, smooth becoming rimose and sometimes scurfy. Apothecia short and narrow, 0.2-1.0 × 0.1-0.3 mm., straight to fre- 
quently curved and flexuous, slightly immersed to adnate; disk black, flat, closed to slightly open; proper margin dull black, rarely with a spurious thalline margin; hypothecium yellowish to brownish black; spores hyaline, fusiform or ellipsoid-dactyloid, 3-7-septate, 16.0-24.0 x 5.0-7.0 $\mu$.

On bark: Thurston Co.: Gate, 1911, Foster 1911 (F).

3. Opegrapha lichenoides Pers. Ann. Bot. Usteri $7: 30$. 1794. Opegrapha varia var. notha Fries.

Thallus white or grayish-white, smooth, thin. Apothecia rather short, oblong, almost circular, 0.2-1.0 × 0.2-0.5 mm., subsessile; disk dull black, soon open, swollen and obliterating the proper margin; hypothecium dark reddish brown; spores hyaline becoming brownish, fusiform, 4-5-septate, 23.2-28.0 x 3.0-8.0 $\mu$.

On bark: Pierce Co.: Mt. Rainier, White River Camp, 3900 ft., 1940, 3106; trail to Mowich Lake, 3500 ft., 1940, 3063b. Thurston Co.: Gate, 1912, Foster (F).

This species is rather easily distinguishable by the swollen disk.

4. Opegrapha protuberans (Stirton) Zahlbr. Cat. Lich. Univ. $2: 233.1924$. O. prosiliens Stirton Grevillea 3:36. 1874.

Thallus white or whitish, thin to very thin, at times almost entirely within the substratum. Apothecia 0.5-2.0 x 0.1-0.2 mm., linear, oblong or ovoid, rarely almost circular, often very irregular in shape, partly immersed to adnate; disk black, very narrow, usually only a crevice, scarcely visible; proper margin black, broad and rounded; hypothecium dark brown to black; spores hyaline, elliptic-fusiform, 3 -septate, third cell the largest, with a gelatinous halo, $13.2-22.4 \times 4-8.3 \mu$.

On bark: San Juan Co.: Turn Island, 1928, 1989; Brown Island, 1940, 3256. King Co.: Alki Point, 1912, Foster 2183 (F). Clallam Co.: Pt. Angeles 1911, Foster (F).

5. Opegrapha varia Pers. Ann. Bot. Usteri 7 :30. 1794.

Thallus white to grayish green, thin, smooth to rough, chinky or powdery, rarely disappearing within the substratum. Apothecia dull black, $0.5-2.0 \times 0.2-0.3 \mathrm{~mm}$., oblong to ovoid, immersed to adnate, straight to slightly curved at maturity; disk open ; proper margin black, thin, prominent, inflexed, usually disappearing; hypothecium brownish black; spores brown when mature, ellipsoid, 3-5-septate, rarely 7-septate, 16.5-32.8 x 4.3-8.4 $\mu$.

On bark: San Juan Co.: Turn Island, 1928, 1990. Pacific Co.: Loomis Lake, 1931, 743. Grays Harbor Co. : 1911, Foster 1477 (F). Clallam Co.: Mt. Angeles, 1911, Foster 1759 (F). Whatcom Co. : Nooksack River, 1914, Herre (F). King Co. : North Bend, 1902, Frye 68 (F).

11. Graphis Adans. Fam. Pl. $2: 11.1763$. 
Thallus thin, rarely entirely disappearing within the substratum. Apothecia lirellae black, elongated, rarely circular, simple or branched, straight or curved, immersed to erumpent; proper margin prominent, thalline thin and inconspicuous; hypothecium hyaline to brownish; paraphyses distinct, unbranched; asci 4-8-spored; spores hyaline, 3-multiseptate.

Alga Trentepohlia.

A. Thallus thin, smooth to slightly wrinkled. 1. G. scripta

A. Thallus thicker, rough and powdery......

2. G. scripta var. pulverulenta

1. Graphis scripta (L.) Ach., Kg1. Vet.-Akad. Nya Handl. 145. 1809. Lichen scriptus L. Sp. P1. 1140. 1753. Opegrapha scripta Ach.

Thallus whitish, olive-buff $(R)$ or dark olive-buff $(R)$, thin, smooth to slightly wrinkled. Apothecia elongated, narrow, 1-5 x 0.2-0.4 mm., straight or curved, simple or branched, scattered or crowded and lying in all directions, immersed becoming erumpent; disk brownish black, sometimes grayish pruinose, closed to open; proper margin black, narrow, raised and covered on the sides by a thin thalline one; hypothecium hyaline; asci 8 -spored; spores hyaline, long ellipsoid, 5-11-septate; loculi lenticular, 20.0-42.9 x 7.0-13.2 $\mu$.

On bark: Grays Harbor Co. : Lake Quinault, 1931, 1209. Clallam Co.: Cedarville, 300 ft., 1940, 2893. Wahkiakum Co.: Cathlamet, 1940, 2854. Kitsap Co.: Hidden Ranch near Bremerton, 213 ft., 1940, 3290. Whatcom Co.: Bellingham, 1920, Weir 16682 (F). King Co.: Seattle, Frye 56 (F). Thurston Co.: Gate, 1912, Foster 2053 (F). Plate I, A.

This species looks very much like pencil markings on the bark.

2. Graphis scripta var. pulverulenta (Pers.) Ach. Syn. Meth. Lich. 82. 1814. Opegrapha pulverulenta Pers. Ann. Bot. Usteri 7 :29. 1794.

Thallus thick, crumbling, and becoming powdery. Apothecia completely immersed or only slightly emergent; otherwise like the species.

On bark: Pacific Co.: Long Beach, 1928, 1925; Chinook, 1940, 2815. Whatcom Co.: Ten Mile Camp, 1914, Herre 4138 (F). Snohomish Co.: Marysville, J. M. Grant (F). Plate I, B.

\section{LECANACTIDACEAE}

Thallus uniformly crustose, little or no differentiation into layers, attached to the substratum by hyphal rhizoids. Apothecia circular, immersed to sessile; proper margin well developed, rudimentary, or entirely lacking, thalline margin present or entirely lacking; asci 2-8-spored; spores hyaline, non-many-septate.

Alga Trentepohlia.

12. Lecanactis Eschw. Syst. Lich., 14. 1824.

Thallus crustose to powdery. Apothecia circular, adnate to sessile; proper margin well developed, thalline lacking; hypothecium brown to 
black; paraphyses branched more or less entwining ; asci 4-8-spored; spores hyaline, 1-15-septate.

1. Lecanactis amylacea var. megaspora (Merrill) Zahlbr., Cat. Lich. Univ. 2:533. 1924. Lecanactis illecebrosa var. megaspora Merrill, Bryol. 16:59. 1913 .

Thallus white or grayish white, thin, powdery to granular. Apothecia circular 0.4-1.2 mm. in diameter, adnate; disk brown or black, sometimes white pruinose, flat to convex or umbonate, becoming irregular in shape; proper margin thin, black, more or less persistent; hypothecium brownish black; asci 8-spored; spores hyaline; fusiform, 1-3-septate, 26.4-32 x 3.3-4.0 $\mu$.

On bark: San Juan Co.: Brown Island, 1940, 3256a. Clallam Co.: near Elwha Post Office, 1911, Foster (F) ; Olympic Hot Springs, 2300 ft., 1914, Foster 2885 ( F).

\section{THELOTREMACEAE}

Thallus uniformly crustose, little or no differentiation into layers, attached to the substratum by hyphal rhizoids. Apothecia circular, single or rarely united in a stroma, immersed or emergent; proper margin well developed and usually surrounded by a thalline one; asci 1-8-spored; spores hyaline or brown, 1-many-septate, or muriform.

Alga Trentepohlia, Phyllactidium, or Heterothallus.

\section{Thelotrema Ach. Meth. Lich. 130. 1803.}

Thallus crustose, partly within the substratum, usually no differentiation into layers. Apothecia small, circular, numerous; proper margin thin but prominent, thalline margin inconspicuous; hypothecium hyaline; paraphyses free, unbranched; asci 1-8-spored; spores hyaline, muriform.

Alga Trentepohlia.

1. Thelotrema lepadinum Ach. Meth. Lich. 130. 1803. Lichen lepadinus Ach. Lich. Suec. Prodr. 30. 1798.

Thallus pale olive-buff $(R)$, cream-buff $(R)$, or various shades of pale gray, smooth to chinky, or areolate, usually thin, sometimes thick. Apothecia circular, small, 0.5-1.0 mm., numerous, at first immersed finally emerging like small warts or craters, the same color as the thallus; disk dark brown or black, white pruinose, partly enclosed, concave to flat; proper margin prominent, thalline margin thin and inconspicuous: asci ustually 1-4-spored, rarely 8 -spored; spores hyaline, ellipsoid-fusiform, muriform, $118.0-126.0 \times 13.2-20.0 \mu$.

On bark, rarely on moss or rock: Clallam Co. : Elwha River Bridge near Pt. Angeles, 244 ft., 1940, 2863, 2883. Thurston Co.: Gate, 1940, 2957. Kitsap Co.: Hidden Ranch near Bremerton, 213 ft., 1930, 1926. King Co.: Grass Mountain Trail, 2200 ft., 1931, 700. San Juan Co.: San Juan Island, 
Friday Harbor, 1906, Fink 217 (F). Clallam Co.: Camp Elk Horn, 2000 ft.. 1907, Frye 39 (W). Clark Co.: Vancouver, 1881, Pringle (W).

This is rather a common bark lichen widely distributed west of the Cascades. It is easily recognized by the numerous, small, pale gray or buffcolored warts or crater-like apothecia.

\section{DIPLOSCHISTACEAE}

Thallus uniformly crustose, upper cortex of poorly developed hyphae, algal and medullary layers poorly differentiated, lower cortex indistinct, attached to the substratum by hyphal rhizoids. Apothecia circular, immersed to adnate; proper margin well developed, thalline margin usually disappearing early; asci 2-8-spored; spores brown or hyaline, 1-septate or muriform.

Alga Protococcus.

14. Diploschistes Norm. Nyt. Mag. Nat. $7: 232.1853$.

Thallus crustose, upper cortex of poorly developed hyphae, algal and medullary layers more or less distinct. Apothecia small, numerous, immersed to adnate; proper margin well developed, thalline margin usually disappearing; hypothecium dark; paraphyses simple, sometimes branched at the tip; asci 2-8-spored; spores brown, muriform.

A. Proper margin thick, radiately striate. 1. D. actinostomus A. Proper margin thin, minutely toothed.

2. D. scruposus

1. Diploschistes actinostomus (Pers.) A. Zahlbr. Hedw. 31:34. 1892. Urceolaria actinostoma Pers. in Ach. Lich. Univ. 288. 1810.

Thallus grayish white, gray or greenish gray, thin to rather thick, smooth to at length areolate. Apothecia minute 0.3-0.5 mm. in diameter, immersed 1-several in an areole; disk black, flat to becoming concave, opening by a pore; proper margin thick, black, grayish pruinose, radiately striate, rarely surrounded by a thin thalline margin; asci 8 -spored; spores brown, ovoid-ellipsoid, muriform, $18-25 \times 12-18 \mu$.

On rocks and rarely on soil: Ferry Co.: North of Republic, $2600 \mathrm{ft}$, 1931, 1402; 1940, 3139. Klickitat Co.: Goldendale, 1909, Foster (F).

2. Diploschistes scruposus (Schreb.) Norm. Con. Prae. Gen. Lich. 20. 1852. Lichen scruposus Schreb. Spicil. Fl. Lips. 133. 1771. Urceolaria scruposa (Schreb.) Ach.

Thallus gray to whitish, olive-buff ( $R$ ), or deep olive-buff $(R)$, areolate, granulate, or warty, forming a thick or thin layer. Apothecia urceolate, 0.2-1.0 $\mathrm{mm}$. in diameter, sunken in thallus; disk black or gray pruinose, deeply concave; proper margin thin, minutely toothed, thalline margin thick, crenulate, often disappearing; asci 4-8-spored; spores brown, ellipsoid, muriform, 19.8-26.4 $\times$ 8.3-10 $\mu$. 
On rock or thin layer of moss or soil over rock: San Juan Co.: Turn Island, 1928, 1991. Chelan Co.: Merritt, 2186, 1076. Yakima Co.: Satus Road, 1054 ft., 1931, 1671a. Garfield Co.: Pomeroy, 1500 ft., 1940, 2734. Upper Naches River Region, 1931, Grant (F). Whitman Co. : Pullman, 2550 ft., C. V. Piper 200 (W).

\section{GYALECTACEAE}

Thallus crustose, usually a thin layer sometimes disappearing within the substratum, rudimentary, very seldom any differentiation into layers, attached to substratum by rhizoids. Apothecia globose to disk shaped, immersed to adnate; proper margin prominent, sometimes covered by the thallus, thalline margin inconspicuous; asci 6-many-spored; spores hyaline, nonseptate, 1-many-septate, or muriform.

Alga Trentepohlia, Phyllactidium, or rarely Scytonema.

A. Spores 1-septate, usually on trees.

15. Microphiale

A. Spores muriform, usually on rocks. 16. Gyalecta

15. Microphiale Zahlbr. in E. \& P. Nat. Pf. Teil. 1, Abt. 1 :125. 1907.

Thallus crustose, usually a thin layer, sometimes disappearing within the substratum, very rudimentary, with seldom any differentiation into layers. Apothecia circular, adnate to sessile; with thick proper margin and a thin thalline one that disappears early; hypothecium hyaline; paraphyses unbranched; asci 8-spored; spores hyaline, 1-septate.

Alga Trentepohlia.

1. Microphiale lutea (Dicks.) Zahlbr. Sitzunb. Kais. Acad. Wiss. Math. Nat. Wien. Classe, 111, Abt. 1: 393. 1902. Lichen luteus Dicks. Fasc. Pl. Crypt. Brit. 1: 11. 1785. Gyalecta lutea (Dicks.) Horn.

Thallus greenish gray or light greenish olive $(R)$, very thin and smooth or somewhat thicker and scurfy. Apothecia small $0.5-1.0 \mathrm{~mm}$. in diameter, circular, adnate to sessile; disk, pinkish cinnamon ( $R$ ), flesh color $(\mathrm{R})$, or pale yellow, flat to convex; proper margin thick, lighter in color than the disk; spores hyaline, fusiform-ellipsoid, 1-septate, 8.2-9.9 × 2.6-3.5 $\mu$.

On trees or decayed logs: San Juan Co.: Turn Island, 1928, 1992.

16. Gyalecta Ach. Kg1. Vet.-Akad. Nya. Hand1. 228. 1808.

Thallus crustose, very thin layer, sometimes disappearing within the substratum, rudimentary, very seldom any differentiation into layers. Apothecia minute to small, at first urceolate, later opening or flattening out, adnate to subsessile; proper margin prominent, sometimes surrounded by an inconspicuous, soon disappearing thalline one; hypothecium hyaline; paraphyses unbranched; asci 8-spored; spores hyaline, muriform.

Alga Trentepohlia.

1. Gyalecta jenensis (Batsch.) Zahlbr. Cat. Lich. Univ. 2: 720. 1924. Peziza jenensis Batsch., Elench. Fung. Contin. prima, 219. 1786. 
Thallus gray or deep olive-buff $(R)$, very thin, smooth to scurfy, sometimes chinky. Apothecia minute 0.2-0.5 mm. in diameter, circular, immersed to adnate; disk pink to reddish brown, concave; proper margin thick, white, thalline one not often seen; spores hyaline, ellipsoid, muriform, 14-18.15 x 6-8.25 $\mu$.

On rocks: Yakima Co. : Boulder Cave, 3000 ft., 1931, 1149.

A rare inconspicuous lichen.

\section{EPHEBACEAE}

Thallus having the appearance of that of a crustose, foliose, or fruticose lichen, consisting of twisted fungal hyphae surrounding and penetrating the filaments of the alga, cortex present or absent. Apothecia globose, superficial or immersed in the alga; proper margin present, thalline one absent; asci 8-spored; spores hyaline, nonseptate or 1-septate.

Alga Scytonema or Stigonema.

A. Thallus with no definite layers; paraphyses thread-like, spores nonseptate.

17. Thermutis

A. Thallus with some indication of definite layers; paraphyses enlarged at the tips; spores 1-septate. 18. Polychidium

\section{Thermutis Fries, Syst. Orb. Veg. $1: 302.1825$.}

Thallus minute, felt-like masses, or cushions of much branched and crowded thread-like filaments, consisting of thin-walled, septate fungal hyphae, extending longitudinally within the sheath of the algae, cortex absent. Apothecia minute, globose, flattened, lateral on the algal filaments, adnate to sessile; disk concave to flat; proper margin thick, colored like the disk; hypothecium hyaline; paraphyses unbranched, thread-like; asci 8-spored; spores hyaline, nonseptate.

Alga Scytonema.

1. Thermutis velutina (Ach.) Fries Syst. Orb. Veg. 1: 302. 1825. Lichen velutimus Ach. Lichenogr. Suec. Prodro. 218. 1798.

Thallus black, brownish black, felt-like masses or cushions of much branched and crowded thread-like filaments. Apothecia rare, minute, not seen on any collections from this state, known to fruit only in the extreme northern regions, lateral 0.4-0.8 mm. in diameter, sessile; disk black, slightly concave; proper margin black, thick, entire, swollen; spores hyaline, ellipsoid, non-septate, 9.0-12.0 × 5.0-7.0 $\mu$.

On rock: San Juan Co.: San Juan Island, Pt. Caution, 1928, 2104. Yakima Co. : Dewey Lake, 4800 ft., 1940, 2789. Ferry Co. : Boyds, 1470 ft., 1789.

18. Polychidium Mass. Mem. Lich. 88. 1853.

Thallus foliose, with an upper and a lower pseudoparenchymatous cortex, an indefinite medulla and algal layer, or pseudoparenchymatous 
throughout. Apothecia small, circular, adnate to sessile; proper margin present, hypothecium hyaline, pale yellow or yellowish brown; paraphyses unbranched, slightly enlarged at the tips; asci 8-spored; spores hyaline, 1 -septate.

Alga Scytonema.

A. Thallus medium sized, foliose, margins bearing minute, white cilia.

1. P. albociliatum

A. Thallus minute, pulvinate, margins not bearing minute, white cilia.

2. P. muscicola

1. Polychidium albociliatum (Desm.) Zahlbr. in E. P. Nat. Pfl. Teil. 1, Abt. 1, 157. 1907. Leptogium albociliatum Desm. Ann. Sci. Nat. Bot. ser. $4,4: 132.1855$.

Thallus foliose, olive-green, or greenish black, medium sized, irregularly lobed, lobes rounded, crisped and crenate, finally lacerate, often minutely granulose in the center, margin bearing minute, white, cilia; below paler, more or less covered with a fleecy nap. Apothecia scattered, 3.0-1.2 $\mathrm{mm}$. in diameter, numerous and often becoming crowded, sessile; disk reddish brown, flat to convex; proper margin thin, soon disappearing; hypothecium hyaline or pale yellow; spores hyaline, spindle-shaped, 1septate, $18.0-26.6 \times 7.6-9 \mu$.

On moss growing over rocks: Ferry Co.: Maye's Butte near Republic, 1913, Foster 2294 (W). Spokane Co.: Spokane, 1906 (F).

2. Polychidium muscicola (Sw.) S. Gray, Nat. Arr. Brit. P1. 1: 402. 1821. Lichen muscicola Sw. Nov. Act. Acad. Upsal. 4: 248. 1784. Leptogium muscicola (Swartz) Fries.

Thallus pulvinate, brown, greenish brown, or a very dark greenish black, minute, irregularly branched, branches interwoven, mostly terete, decumbent. Apothecia appressed, 0.2-0.8 mm. in diameter, circular, sometimes irregular adnate to sessile; disk brownish red, flat to convex; proper margin thin, slightly paler than the disk; hypothecium yellowish brown; spores hyaline, oblong-fusiform, 1-septate, $17-30 \times 5-8 \mu$.

On mossy rocks or soil: Washington Territory, Suksdorf (F), Ex. Herb. of C. E. Faxon.

\section{COLLEMACEAE}

Thallus usually foliose, varying from subcrustose to fruticose; gelatinous when moist, brittle when dry; with or without distinct cortices or having only an upper pseudocortex, inside hyphae ramify through the algal colony; adhering closely to the substratum or attached by rhizoids. Apothecia globose or disk-like, marginal or scattered, immersed to adnate, both proper and thalline margins present or only a proper one; asci 8 -spored; spores hyaline, nonseptate, many-septate or muriform.

Alga Nostoc. 
A. Thallus lacking distinct upper and lower cortices. 19. Collema

A. Thallus with a distinct cortex on the upper surface only, or on both surfaces.

20. Leptogium

19. Collema Wigg. Primit. Fl.-Holsat. 89. 1780.

Thallus foliose, thin; gelatinous when moist, brittle when dry; often hyphae grow close together to form an upper pseudocortex, no true cortices. Apothecia small, circular, immersed, adnate or sessile; proper margin present, thalline margin often very prominent; hypothecium hyaline to brown; paraphyses unbranched, agglutinated; asci 8-spored; spores hyaline, muriform.

A. On soil or rock.

B. Apothecia crowded, oblong-ellipsoid. 2. C.crispum

B. Apothecia scattered, spores ellipsoid.

3. C.furvum

A. On bark or old wood.

C. Spores submuriform, quadrangular or ellipsoid.........4. C.verruciforme

C. Spores not submuriform, fusiform or acicular................1. C.nigrescens

1. Collema nigrescens (Huds.) DC. Lam. et DC. F1. France 3rd ed., 2:384. 1805. Lichen nigrescens Huds. Fl. Anglica Ed. 2. 450. 1762. Synechoblastus nigrescens (Huds.) Trev.

Thallus when dry olive-green or greenish black, same color when moist; lobes small, rounded, flattened, beset with pustules, often ridged, margins entire, ascending, closely adhering to the substratum. Apothecia often numerous, scattered over the upper surface, often crowded towards the center of the thallus, $2.0-8.0 \mathrm{~mm}$. in diameter, circular, sessile; disk reddish brown or pale pink, flat to convex; thalline margin thin; hypothecium pale yellow; spores hyaline, fusiform or acicular, sometimes slightly curved, 5-7septate 49.4-70.0 × 3.8-6.0 $\mu$.

On bark: San Juan Co.: San Juan Island, 1906, Fink 235 (W). Grays Harbor Co. : Westport, 1908 Foster 6651/2 (W).

2. Collema crispum (L.) Wigg. Prim. Fl. Holsat. 89. 1780. Lichen crispus Linn., Fl. Suec. 2nd ed., 406. 1755.

Thallus when moist, dark olive $(R)$ or metal bronze $(R)$, when dry dark brown to black; lobes small, round, rather irregular, margins raised, dentategranulate, often the central part covered by numerous apothecia. Apothecia 0.5-1.0 $\mathrm{mm}$. in diameter, circular, adnate to subsessile; disk burnt sienna $(R)$, concave; thalline margin, granular crenulate; hypothecium brownish; spores hyaline, oblong-ellipsoid, 3-septate, muriform when mature, 16.5-23.1 x 9.9-13.2 $\mu$.

On soil or rock: San Juan Co. : San Juan Island, Friday Harbor, 1928, 1993; Roche Harbor, 1928, 1876.

3. Collema furvum (Ach.) DC. in Lam. et DC. F1. Fran. Ed. 3, 2: 385. 1805. Lichen furvus Ach. Lich. Suec. Prodro. 132. 1798. 
Thallus when moist dark olive green $(R)$, when dry grayish black; lobes small, round, margins erect, undulate, surface granular. Apothecia 0.2-1.0 $\mathrm{mm}$. in diameter, circular, scattered, adnate; disk brown, concave to flat; thalline margin prominent; hypothecium brownish; spores hyaline, ellipsoid, 3 -septate, muriform when mature, $17-25 \times 8-12 \mu$.

On soil or rock: Ferry Co.: between Curlew and Danville, $1773 \mathrm{ft}$., 1931, 1413. Washington Territory, Cascade Mountains, 1883, Brandegee $19(\mathrm{~T})$.

4. Collema verruciforme (Ach.) Nyl. Memoir Soc. Sci. Nat. Cherbourg, $3: 165$. 1855. Collema furvum var. verruciforme Ach. Lich. Univ. 650. 1810.

Thallus when moist vivid green $(R)$, when dry dark brown; lobes small to minute, crowded, smooth, margins ascending, crenate. Apothecia minute never more than $0.3 \mathrm{~mm}$. in diameter, circular, immersed to adnate; disk reddish brown, deeply concave, blood red when moist; thalline margin thick, brown when dry, greenish when moist; hypothecium hyaline to pale yellow; spores hyaline, quadrangular or ellipsoid, becoming muriform, 11.0-21.0 $\times$ 7.6-11.4 $\mu$.

On bark or old wood: Chelan Co.: shores of Lake Wenatchee, $1800 \mathrm{ft}$., 1931, 1063.

20. Leptogium S. Gray, Nat. Arr. Brit. P1. $1: 400.1821$.

Thallus foliose, or rarely fruticulose; gelatinous when moist, brittle when dry; distinct cortex on the upper surface only, or on both surfaces. Apothecia often lacking, very small, circular, immersed, adnate, or sessile; proper margin present, thalline margin sometimes disappearing; hypothecium hyaline to brownish; paraphyses unbranched, agglutinated; asci 8 spored; spores hyaline, 1-many-septate, or muriform.

A Lobes narrow becoming involute tubular towards the tips.

3. L.palmatum

A. Lobes not as above.

B. Thallus small.

C. Margins ascending, denticulate or fringed.

D. Lobes densely crowded, forming a cushion-like mass, usually sterile......

2. L. lichenoides var. pulvinatum

D. Lobes not densely crowded, not forming a cushion-like mass, usually fruiting..................................................... Lichenoides

C. Margins somewhat ascending, undulate, sparsely, white pilose.

4. L.pilosellum

B. Thallus larger.

E. Upper surface smooth, somewhat wrinkled. 5. L.pulchellum

E. Upper surface densely coralloid-granulose.

6. L.tremelloides 
1. Leptoginm lichenoides (L.) Zahlbr., Cat. Lich. Univ. 3: 136. 1924. Tremella lichenoides Linn. Sp. P1. 1157. 1753. Leptogium lacerum (Swartz) S. Gray.

Thallus neutral gray $(R)$, reddish brown, or brown, small to medium sized, surface wrinkled and pitted, lobes laciniate, margins ascending denticulate or fringed, sometimes sinuate; below slightly lighter. Apothecia rare, scattered, 0.2-0.6 $\mathrm{mm}$. in diameter, circular, subsessile; disk when dry concolorous with the thallus, when moist dull reddish brown, flat to concave; thalline margin concolorous with the thallus; hypothecium hyaline to cloudy; spores hyaline; ovoid-ellipsoid, muriform, multi-septa, 28.4-52.8 x 16.5-19.8 $\mu$.

On mosses or on a thin layer of soil over rock: Chelan Co.: Rainbow Trail near Stehekin, 3500 ft., 1931, 1260a. Yakima Co.: Naches Ranger Station, 2400 ft., 1940, 3326. Klickitat Co.: Goldendale, 2000 ft., 1931, 1687a. San Juan Co.: Waldron Island, 1906, Fink (F). Clallam Co.: Pt. Angeles 1914, Foster (F). Oregon Boundary Commission, 1858, Dr. Lyall $(\mathrm{T})$.

2. Leptogium lichenoides var. pulvinatum (Hoffm.) Zahlbr. Cat. Lich. Univ. 3: 142. 1924. Collema pulvinatum Hoffm. Deutschl. F1. 104. 1796. Leptogium lacerum var. pulvinatum (Hoffm.) Mont.

Thallus brown, very small, lobes erect, crowded, margins denticulate and fringed much like the species, in cushion-like masses, usually sterile.

On mosses or on a thin layer of soil over rocks: San Juan Co.: Brown Island, 1940, 3199. Chelan Co.: Merritt, 3500 ft., 1931, 1075. Whitman Co.: Steptoe Butte, 3700 ft., 1940, 2743.

The variety is distinguished from the species by the cushion-like habit.

3. Leptogium palmatum (Huds.) Mont. in Webb \& Berth. Hist. Nat. 3 : 128. 1840.

Lichen palmatus Huds. Fl. Angelica, 2nd ed., 2. 536. 1778.

Thallus when dry, neutral gray ( $R$ ), brown, or fuscous ( $R$ ), when moist, grape green $(R)$; medium to large, thin, surface smooth to faintly reticulate or pitted, lobes deeply divided, lobules long and narrow, resembling fingers, tubular at the tips, involute, margins with scattered teeth; below slightly paler, more wrinkled. Apothecia often numerous, scattered, very small, 0.2-0.8 mm. in diameter, circular, sessile; disk fawn colored $(\mathrm{R})$ or reddish brown, concave; thalline margin smooth, elevated; hypothecium hyaline to pale yellow; spores hyaline, ovoid-ellipsoid, muriform, multi-septate, $36.3-46.2 \times 14.6-16.5 \mu$.

On soil: San Juan Co.: San Juan Island, Pt. Caution, 1940, 3270; Brown Island, 1940, 3198. Clallam Co. : Elwha River, 1940, 2879. Kitsap Co. : Hidden Ranch near Bremerton, 213 ft., 1940, 3293. Clallam Co. : near Pt. Angeles, 2000 ft., 1914, Foster 2768 (F). King Co.: Snoquainie Falls, 1906 Frye (F): Seattle, Univ. of Wash. campus, 1905, Frye 5 (W). 
A very common Leptogium west of the Cascades, perhaps the most common Leptogium in the state. Easily identified by the tubular ends of the lobes.

4. Leptogium pilosellum Merrill Bryol. $14: 38$. 1911.

Thallus when dry, dark ivy green ( $R$ ), when moist, dark green $(R)$ or Cedar green (R) ; orbicular, medium sized, surface smooth to scabrous, expanded to somewhat appressed, margins undulate, at times white pilose; below the same color as upper surface, sparsely covered with white rhizoids. Apothecia $0.5-0.8 \mathrm{~mm}$. in diameter, circular, adnate to subsessile; disk bright red to reddish brown, flat; thalline margin white pilose; hypothecium hyaline to cloudy; spores hyaline, fusiform-ellipsoid, 1-septate, 13.2-23.1 x 6.9-7.3 $\mu$.

On mosses over rock: Chelan Co.: Merritt, 2186 ft., 1931, $1078 b$. Klickitat Co.: Goldendale, 2000 ft., 1931, 1687; Goldendale, Foster 1186, Merrill's Type (F).

5. Leptogium pulchellum (Ach.) Nyl. Memoir Soc. Sci. Nat. Cherbourg 5: 32. 1857. Collema pulchellum Ach. Syn. Lich. 321. 1814.

Thallus, when dry, deep grayish blue green, when moist, green ; medium sized to large, surface smooth to somewhat wrinkled, lobes round imbricated, margins wavy; below paler much wrinkled. Apothecia rather numerous, scattered, 0.5-2.0 mm. in diameter, circular, sessile to subpedicellate; disk reddish brown, flat to slightly concave; thalline margin thin concolorous with the thallus; hypothecium brownish; spores hyaline, ellipsoid, muriform, $18.0-34.2 \times 7.0-9.9 \mu$.

On bark and rock: Pacific Co.: Fort Canby, 1930, 486. Reported from the state by Fink (1935) and by Herre (1917) from the lowlands of Whatcom Co.

6. Leptogium tremelloides (L.) S. Gray, Nat. Arr. Brit. Pl. 1 :400. 1821. Lichen tremelloides Linn. Suppl. P1. 450. 1781.

Thallus lead colored, same color whether dry or moist, medium sized, suborbicular, closely attached to the substratum, surface coralloid-granulose, lobes ascending, imbricated, margin entire to undulate; below lighter in color. Apothecia $0.5-1.0 \mathrm{~mm}$. in diameter, circular to irregular, sessile to subpedicellate; disk reddish brown, concave becoming convex; thalline margin entire to irregular, sometimes disappearing, concolorous with the thallus; hypothecium hyaline to brownish; spores hyaline, ovoid-ellipsoid, muriform, $18-28 \times 8-11 \mu$.

On bark and rock: Whatcom Co.: Sumas Mountain, Herre (1917); reported from the state by Fink (1935).

\section{PANNARIACEAE}

Thallus foliose, squamulose, or crustose; dorsi-ventral ; upper cortex of pseudoparenchymatous tissue, algal layer and medulla always present, lower cortex sometimes lacking; dark hypothallus ordinarily present; usually 
attached to the substratum by rhizoids. Apothecia disciform, marginal or scattered over the upper surface, immersed to sessile, lecanorine or biatorine; asci 8-spored; spores hyaline, nonseptate or 1-3-septate.

Alga Nostoc, Scytonema, Gloeocapsa, or Protococcus.

A. Thallus definitely foliose throughout, veins prominent....25. Hydrothyria A. Thallus not definitely foliose throughout, veins not prominent.

B. Alga is a blue-green.

C. Spores septate, alga Scytonema and Gloeocapsa......23. Massalongia

C. Spores nonseptate, alga Gloeocapsa.

D. Lecanorine.

D. Biatorine.

22. Pannaria

21. Parmeliella

B. Alga is a green. 24. Psoroma

21. Parmeliella Müll. Arg. Mem. Soc. Phys. et Hist. Nat. Geneve. $16: 376.1862$.

Thallus crustose or squamulose, almost foliose at the periphery, pseudoparenchymatous cortex well developed on the upper surface only, definite algal and medullary layers; a dark hypothallus present. Apothecia disciform, immersed to adnate; proper margin present, thalline margin absent; hypothecium hyaline or faintly colored; paraphyses unbranched; asci 8-spored, hyaline, nonseptate.

Alga Gloeocapsa.

A. Thallus a mass of steel-blue or slate-blue $(R)$ granules, sterile.

A. Thallus not as above, squamulose.

1. P. cyanolepra

B. Margins of squamules warty or sorediate.

2. P. lepidiota

B. Margins of squamules not warty or sorediate, but often lighter in color.

3. P. microphylla

1. Parmeliella cyanolepra (Tuck.) Herre, Proc. Wash. Acad. Sci. $12: 151$. 1910.

Pannaria cyanolepra Tuck. Lich. Calif. 17. 1866. Pannaria lepidiota var. cyanolepra Tuck.

Thallus a mass of steel-blue to slate-blue $(R)$ granules; the writer has studied no fruiting material collected in this state. The following description was made from Tuckerman's Type 4626 (F) : apothecia 0.5-1.0 mm. in diameter, immersed to adnate; disk dark reddish brown to black, flat becoming convex and excluding the thin proper margin; hypothecium hyaline; spores hyaline; ellipsoid, nonseptate, 8-10 x 14-21 $\mu$.

On moss, soil, or rock: Chelan Co. : 2186 ft., 1931, 1077. Columbia Co. : Blue Mountains, 3500 ft., 1931, 1630. Whatcom Co.: Sumas Mountain, $1800 \mathrm{ft} .$, Herre (1917).

2. Parmeliella lepidiota (Somrft.) Wainio Term. Fuz., 22 :308. 1899. Lecidea carnosa var. lepidiota Somrft. Suppl. Fl. Lappon. 174. 1826. Pannaria lepidiota (Somrft.) Th. Fr. 
Thallus of small tawny-olive or brown squamules, imbricated, margins ascending, crenate, well covered with steel-blue to slate-gray warts, often gray sorediate, near the center squamules often disappearing and a granulose crust appearing; below brown or blackish; hypothallus indistinct. Apothecia $0.5-1.0 \mathrm{~mm}$. in diameter, adnate to sessile; disk reddish brown to black, flat becoming convex, the thin proper margin soon disappearing; hypothecium pale yellow; spores hyaline, ovoid-ellipsoid, sharply pointed, more pronounced at one end, nonseptate, $16.5-26.4 \times 6.6-11.5 \mu$.

On soil, moss, and rock: Pacific Co.: Beard's Hollow, 1931, 782. Chelan Co.: White Rock Camp, $2200 \mathrm{ft} ., 1057$; Rainbow Trail, $3800 \mathrm{ft.}, 1251 a$; Dirty Face Mt., 3500 ft., 1098. Clallam Co.: Bird's Port Angeles, 1914, Foster 2664 (F).

This species is rather easily distinguished by the steel-blue or slate-gray warts and gray soredia on the margins of the squamules.

3. Parmeliella microphylla (Swartz) Müll. Arg., Flora 72:507. 1889.

Lichen microphyllus Swartz, Vet-Akad. Handl. 301. 1791.

Pannaria microphylla Mass.

Thallus of very small light yellowish-olive ( $R$ ), fawn color (R), or brown squamules, imbricated, margins lighter colored, ascending; below dark brown or black. Apothecia $0.4-1.0 \mathrm{~mm}$. in diameter, adnate to subsessile; disk reddish brown or black, flat to convex; proper margin soon excluded; hypothecium hyaline; spores hyaline, ellipsoid, nonseptate, $14.0-23.1 \times 5.0-6.0 \mu$.

On soil, moss, and rock: Chelan Co.: Merritt, 2186 ft., 1931, 1085. Clallam Co.: Port Angeles, 1914, 2769 (F). Mason Co.: Mt. Elinor, 4000 ft., 1912, Foster 2110 (F).

The very small squamules and light-colored margins are the determining characters for this species.

22. Pannaria Del., in Bory, Dict. Class. Hist. Nat. 13.20. 1828.

Thallus granulose, squamulose or foliose; a dark hypothallus often present or a white cobweb one, of felted hyphae, a well-developed typical cortex on the upper surface, definite algal and medullary layers, and a thin lower cortex. Apothecia disciform, adnate to sessile; proper margin present, thalline margin concolorous with the thallus; hypothecium hyaline, faintly colored, or brownish; paraphyses rarely branched; asci 8-spored; spores hyaline, nonseptate.

Alga Gloeocapsa.

A. Usually on soil or moss, rarely on rock or bark; hypothallus when present white, thalline margin drab colored; spores minutely warted.

1. P. pezizoides

A. Usually on bark, rarely on soil or rock; hypothallus black, thalline margin grayish white; spores smooth.

2. P. rubiginosa 
1. Pannaria pezizoides (Weber) Trevis. Lich. Vene. No. 98. 1869. Lichen pezizoides Weber. Spicil. Fl. Goet. 200. 1778.

Pannaria brunnea (Swartz) Mass.

Thallus of small to very small squamules; when dry grayish brown or sepia ( $R$ ), when moist greenish blue, imbricated, often crowded into a granulose mass, margins crenate; hypothallus when present white cobweblike. Apothecia often large and numerous, irregular in shape due to crowding, adnate; disk orange-cinnamon ( $\mathrm{R})$, brick-red, or reddish-brown, flat to convex; thalline margin drab colored $(R)$, crenulate; hypothecium pale yellow, spores hyaline, ellipsoid to ellipsoid-pointed, minutely warted, nonseptate, $24.7-28.0 \times 8.9-11.1 \mu$.

On soil or moss, more rarely on rock: Grays Harbor Co.: Lake Quinault, 300 ft., 1931, 1203. Clallam Co.: Lake Crescent, 483 ft., 1931, 1036. Pierce Co. : Mt. Rainier, Berkeley Park, 6000 ft., 1931, 623; trail to Seattle Park, 4000 ft., 1940, 3055. Cascade Mountains, 1925, Grant (F). Clallam Co. : Port Angeles, 1911, Foster 1772 (W).

The usually persistent crenulate drab-colored margin helps to distinguish this species.

2. Pannaria rubiginosa (Thunbg.) Del. in Bory, Dict. Class. Hist. Nat. $13: 20.1828$.

Lichen rubiginosus Thunbg. in Ach. Lich. Suec. Prodro. 99. 1798.

Thallus orbicular, of smoke gray $(R)$, greenish gray, or various shades of pale yellow squamules, closely attached to the substratum, imbricated margins deeply divided, raised, grayish white granular-crenate. Apothecia 0.5-1.5 mm. in diameter, numerous and crowded, sessile; disk red to reddish brown, flat to slightly concave; when present thalline margin is prominent, grayish white crenulate, concolorous with the thallus; hypothecium pale yellow or brownish; spores hyaline, ellipsoid, nonseptate, 11.2-16.5 x 6-11 $\mu$.

On bark, more rarely on rock: Pacific Co.: Long Beach, 1928, 1925a; Chinook, 1940, 2816. Chelan Co.: Stehekin, 1140 ft., 1931, 1285. Pierce Co.: Mt. Rainier, trail to Seattle Park, 4000 ft., 1940, 3046. Clallam Co.: Port Angeles, Bird's, 1914, Foster 2664 (W). Grays Harbor Co.: Westport, 1908, Foster (W). Determined as Pannaria brunnea Mass. by G. K. Merrill.

This species is easily distinguished by the grayish white margins of the squamules and the apothecia.

23. Massalongia Korb. Syst. Lich. Ger. 109. 1855.

Thallus squamulose, foliose, at times almost crustose, with a dark rhizinose hypothallus, pseudoparenchymatous cortex on the upper surface, decorticate on the lower, a thin algal layer and a spongy medulla. Apothecia disciform, sessile; proper margin thin and pale, thalline margin absent; hypothecium hyaline to pale yellow; paraphyses septate, coherent, asci 8-spored; spores hyaline, 1-septate. 
Alga Scytonema and Gloeocapsa.

A very small genus. A single species within the state.

1. Massalongia carnosa (Dicks.) Körb. Syst. Lich. Ger. 109. 1855.

Lichen carnosus Dicks. Fasc. P1. Crypt. Brit. 2:21. 1790.

Pannaria carnosa (Dicks.) Rabh.

Thallus of small squamules, when dry, brown, lighter colored at the edge, when moist, the whole squamule is bluish green, lobate, suberect, imbricated, granular-crenate; below whitish. Apothecia small $0.2-0.5 \mathrm{~mm}$. in diameter, sessile; disk dark red, flat, proper margin thin and entire; hypothecium pale yellow; spores hyaline, oblong-fusiform, to ellipsoid, 1-septate, $23.1-24.8 \times 5.6-7.0 \mu$.

On rock or soil among mosses: Yakima Co.: American River Camp, 2800 ft., 1931, 944. Clallam Co.: Olympic Hot Springs near Port Angeles, 1914, Foster 2869c (F).

\section{Psoroma Nyl. Mem. Soc. Sci. Nat. Cherbourg $3: 175.1855$.}

Thallus squamulose; hypothallus absent; upper cortex of pseudoparenchyma or of interwoven hyphae, definite algal and medullary layers, thin lower cortex of densely interwoven hyphae. Apothecia medium sized to large, disciform, adnate to sessile; both proper and thalline margins present, thalline concolorous with the thallus; hypothecium hyaline or pale yellow; paraphyses unbranched, coherent, asci 8-spored; spores hyaline, nonseptate.

Alga Protococcus.

1. Psoroma hypnorum (Vahl.) S. Gray, Nat. Arr. Brit. P1. 1 :445. 1821. Lichen hypnorum Vahl. Icon. P1. Dan. 6; fas. 16. Pannaria hypnorum (Vahl.) Körb. Parmelia hypnorum (Vahl.) Ach.

Thallus of yellow, reddish brown or grayish squamules, minute, rounded, granulate, crenate, ascendant, often imbricated, closely attached to the stems and leaves of the mosses among which they grow, usually concealed somewhat by the moss, paler below. Apothecia medium sized to large, 2.0-5.0 $\mathrm{mm}$. in diameter, numerous, circular, sometimes irregular in shape, sessile; disk red-brown, flat to concave; thalline margin thin, conspicuous, granulose, concolorous with the thallus; spores hyaline, ovoid-ellipsoid, nonseptate, $16.0-22.8 \times 7.6-12.0 \mu$.

On soil, also over moss and twigs: Pierce Co.: Mt. Rainier, trail to Moraine Park, 3300 ft., 1940, 2986. Yakima Co.: Dewey Lake, 4800 ft., 1940, 2772. Mt. Rainier, 6500 ft., 1895, Piper 525 (W). Clallam Co.: Olympic Hot Springs, 1914, Foster 28696 (F).

\section{Hydrothyria Proc. Essex. Inst. 1 :188. 1853.}

Thallus foliose, thin membranaceous; pseudoparenchymatous cortex better developed on the upper surface than the lower; a partially distinct algal layer and a medullary layer of compact filaments are present; without rhizoids. Apothecia submarginal near the tips of the lobes, sessile, biatorine; 
hypothecium hyaline or light brown; paraphyses thick, several septate, tips brownish; asci 8-spored, hyaline, 3-septate. A monotypic genus.

Alga Nostoc.

1. Hydrothyria venosa Russ. Proc. Essex. Inst. 1 :188. 1853.

Thallus when dry, brittle, dark brown or black, when moist, rather elastic, light seal brown $(R)$; laciniate, lobes irregularly cut, margins deeply incised, veins prominent on the lower surface, also evident on the upper. Apothecia submarginal or subterminal, $0.6-3.0 \mathrm{~mm}$. in diameter, sessile ; disk reddish brown or almost black, flat to convex, the proper margin pale in color, torn-dentate, and disappearing; spores hyaline, fusiform, 3-septate, $26.4-29.7 \times 6.6-9.0 \mu$.

On rock in mountain streams: Pierce Co.: Mt. Rainier, Berkeley Park, 6000 ft., 1931, 612; 1940, 3010; Seattle Park, 4000 ft., 1940, 3060; Van Trump Park, 5000 ft., 1942, 3572. Jefferson Co.: Queets River Valley, $5000 \mathrm{ft} ., 1907$, Frye 15 (W).

The unusual habitat, in swiftly flowing mountain streams, makes this an easy lichen to recognize in the field.

\section{STICTACEAE}

Thallus foliose, small to very large; dorsi-ventral; with definite upper and lower cortices and well-developed algal and medullary layers; upper surface smooth or reticulate-netted; below tomentose or villous, interspersed with cyphellae or with naked, light-colored spots; closely or loosely attached to the substratum by rhizoids. Apothecia marginal or scattered, adnate, sessile, or subpediculate, with either a proper or a thalline margin; asci 8-spored; spores brownish, 1-many-septate.

Alga Nostoc, Palmella, or Protococcus.

A. Lower surface of thallus without cyphellae.

A. Lower surface of thallus with cyphellae.....

26. Lobaria

26. Lobaria Schreb. Gen. P1. $2: 768.1789$.

Thallus often large, foliose, broadly expanded, horizontal ; below usually villous or tomentose, cortex interrupted by naked, light-colored, inflated spots, loosely attached to the substratum. Apothecia medium sized, circular, sessile, or subpedicellate, generally with a thalline margin; hypothecium hyaline or brownish; paraphyses unbranched, septate; asci 8-spored; hyaline or brown, 1-9-septate.

Alga Protococcus or Nostoc.

A. Thallus always reticulate-ribbed, below with large white, inflated spots, veins of brown hairs between them.

B. Lobes narrow elongated, tips obtuse or retuse-truncate, margins and ridges often sorediate or isidiose.

2. L. pulmonaria

B. Lobes broad, often much divided, tips rounded, margins and ridges, undulate-crenate, often fringed with tooth-like lobules.

1. L. oregana 
A. Thallus rarely reticulate-ribbed, usually pitted and wrinkled, below with smaller, inflated, flat or sunken white spots, scattered through the dense tomentum.

C. Soredia marginal and scattered, smooth on upper surface.

4. L. verrucosa

C. Soredia not on the margins, but scattered, villous on upper surface.

3. L. Hallii

1. Lobaria oregana (Tuck.) Müll. Arg. Flora $72: 364.1889$.

Sticta oregana Tuck. Bull. Torr. Bot. Club $5: 20.1874$.

Thallus deep olive-buff $(R)$ or raw umber $(R)$, medium sized to large, coriaceous or membranaceous, pitted and reticulate-ribbed, lobes broad, often much divided, tips rounded, margin undulate-crenate, often fringed with tooth-like lobules; below honey-yellow (R) or brownish-olive (R), covered with a reticulation of fine hairs interspersed with large, naked, inflated, or blister-like spots. Apothecia scattered, 1.0-4.0 mm. in diameter, sessile to subpedicellate; disk cinnamon, bay (R), chestnut-brown, or almost black, flat to convex; thalline margin thin, crenate, concolorous with the thallus, finally disappearing; spores hyaline, 3-septate, fusiform or acicular, 54.4-62.7 x 3.9-5.2 $\mu$.

On bark: King Co.: Grass Mountain Trail, 1931, 2200 ft., 1227. KingKittitas Co.: Snoqualmie Pass, 3010 ft., 1931, 810. Pierce Co.: Mt. Rainier, trail to Seattle Park, 4000 ft., 1940, 3051; Mazama Ridge, 4000 ft., 1942, 3594. Clallam Co.: Port Angeles, Coleman's, 1914, Foster 2539 (W). Whatcom Co.: Ruth Mountain, 5500 ft., 1928, Sunquist 2002 (W). King Co.: Summit, 1907, Foster (W). Skamania Co.: Little Soda Springs Camp Ground, 1933, Lawrence (W). Plate I, C.

A common bark lichen in the mountains, west of the Cascades. It is often confused with $L$. pulmonaria, but it can be distinguished by the undulate-crenate margin of the thallus, which is often fringed with toothlike lobules; soredia entirely lacking, often present on L. pulmonaria.

2. Lobaria pulmonaria (L.) Hoffm. Deutschl. Fl.:146. 1795. Lichen pulmonarius Linn., Spec. P1. 1146. 1753. Sticta pulmonaria Birola, Fl. Ascon. 2:188. 1808 .

Thallus tawny olive ( R), antique brown (R), chamois ( R), or greenish gray, medium sized to very large, thin leathery or coriaceous, pitted and reticulate-ribbed, lobes narrow, elongated, tips obtuse or retuse-truncate, margins and ridges often sorediate or isidiose; below chamois $(R)$ or brown, covered with a close nap of tomentum, interspersed with large, naked, inflated, or blister-like spots. Apothecia rather rare, scattered, or submarginal, $1.0-6.0 \mathrm{~mm}$. in diameter, sessile to subpedicellate; thalline margin thin, entire to wrinkled, concolorous with the thallus, finally disappearing; spores hyaline, cymbiform, 1-3-septate, 23.2-33.0 x 6.6-7.2 $\mu$.

On bark and rock: Pierce Co.: Silver Springs, 2600 ft., 1940, 3110; White River Camp, 3000 ft., 1928, 1847. King-Kittitas Co.: Snoqualmie 
Pass, 3010 ft., 810a. Pend Oreille Co. : Metaline Falls, 2600 ft., 1931, 1474. San Juan Co.: San Juan Island, Friday Harbor, 1906, Fink 225 (W). Clallam Co.: Olympic Hot Springs, 1914, Foster 2867. (W). Skamania Co.: Little Soda Springs Camp Ground, 1933, Lawrence (W). King Co.: Seattle, 1893, Piper 531 (W). Plate I, D, and Plate II, A.

A rather common large foliose lichen, usually found on bark, rarely on rock, easily confused with $L$.'oregana.

3. Lobaria Halli (Tuck.) Zahlbr. Cat. Lich. Univ. 3 :321. 1925.

Sticta Hallii Tuck. Proc. Am. Acad. Arts and Sci. 12 :168. 1877.

Thallus Isabella color $(R)$ or deep olive buff $(R)$, small to medium sized, coriaceous-cartilagineous, reticulately pitted, with shallow cracks, granulose villous, lobes wide, rounded and entire, beset with scattered gray soredia, usually not on the margins; below clay color ( $R$ ) or light pinkish cinnamon $(R)$, covered with a reticulated tomentum interspersed with irregular white, naked spots. Apothecia rather rare, scattered or submarginal, $0.3-3.0 \mathrm{~mm}$. in diameter, sessile; disk flat to convex; thalline margin pilose, concolorous with the thallus; spores brown, cymbiform, 1-septate,

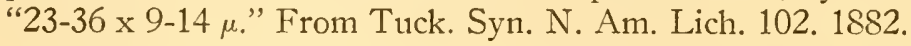

On bark or soil: Thurston Co.: Gate, 1912, Foster 1895 (F). Washington Territory, Suksdorf (F). Type collected in Oregon 1871 by E. Hall. No fruiting specimens from the state have been seen by the writer.

The more or less hairy upper surface and the veined lower surface separate sterile specimens of $L$. Hallii from $L$. verrucosa.

4. Lobaria verrucosa (Huds.) Hoffm. Deutsch1. F1. 2:146. 1796. Lichen verrucosus Huds. Fl. Ang. 445. 1762. Sticta scrobiculata (Scop.) Ach. Sticta verrucosa (Huds.) Fink.

Thallus dark olive-buff $(R)$, deep colonial-buff $(R)$, or Isabella color $(\mathrm{R})$, medium sized, coriaceous, suborbicular, pitted and wrinkled, rarely reticulate-ribbed, lobes rounded, margins entire, often much beset with groups of gray or white soredia, soredia more rarely scattered over the surface; below clay color $(\mathrm{R})$, light pinkish cinnamon ( $\mathrm{R})$, or becoming almost black, covered with a dense tomentum, interspersed with scattered, small, naked, white, blister-like, flat, or sunken spots. No fruiting material, collected in this state, has been seen by the writer. Tuckerman studied no fertile material from the United States.

On rocks, soil, and bark: San Juan Co.: San Juan Island, 1930, 501; Pt. Caution, 1940, 3266; Brown Island, 1940, 3211; Orcas Island, Mt. Constitution, 2500 ft., 1928, 1999. Pacific Co.: Chinook, 1940, 2813. San Juan Co.: San Juan Island, Friday Harbor, 1906, Fink 16 (W). Clallam Co.: Port Angeles, Coleman's Butte, 2000 ft., 1914, Foster 2641 (W). Thurston Co.: Gate, 1911, Foster 1931 (IV). King Co.: Seattle, Parker 29 (W).

This species is easily separated from L. Hallii by the spore differences. 
27. Sticta Schreb. in Linn. Gen. P1. Sth ed., 2 :768. 1791.

Thallus small to medium sized, foliose, horizontal or often somewhat ascending, upper surface usually sorediate or isidiose; below villous or tomentose, cortex interrupted by cyphellae; closely or loosely attached to the substratum. Apothecia when present medium sized, in some species very rare or perhaps never produced, circular or irregular in shape, marginal or scattered, adnate, sessile, or subpedicellate, thalline margin present or absent; hypothecium hyaline or brownish; paraphyses unbranched, septate; asci 8-spored; spores hyaline or brown, 1-7-septate.

Alga Nostoc or Palmella.

A. Cyphellae sorediate, elevated.

B. Soredial cyphellae yellow, upper surface yellow sorediate...2. S. crocata

B. Soredial cyphellae white, upper surface sparingly gray sorediate.

A. Cyphellae naked, concave.

1. S. anthraspis

C. Upper surface abundantly covered with brown or black isidia.

3. S. fuliginosa

C. Upper surface not bearing isidia, but abundantly or sparingly covered with grayish soredia.

4. S. limbata

C. Upper surface smooth, margins isidiose........................5. S. quercizans

1. Sticta anthraspis Ach. Meth. Lich. 280. 1803.

Thallus Isabella color $(\mathrm{R})$, cinnamon brown, or greenish gray, medium to large sized, cartilagineous-coriaceous, lobes broad, margins coarsely crenate or sinuate, sometimes lacerate, rather deeply reticulate-pitted, sometimes gray sorediate especially on the ridges; below chamois ( $R)$, honey-yellow, or smoky-brown, darker near the center, covered with a thin tomentum, interspersed with white sorediate cyphellae; loosely attached to the substratum. Apothecia scattered, 1-4 mm. in diameter, sessile; disk chestnut-brown or almost black, flat to slightly convex; thalline margin thin, entire or sometimes denticulate, often disappearing; spores hyaline, fusiform, straight, 1-3-septate, 23.1-26.4 x 6.6-7.6 $\mu$.

On bark, on soil among mosses, or on rock. Pacific Co.: Loomis Lake, 1930, 439, 1940, 2851; Long Beach, 1928, 1846; Chinook, 1940, 2799. Clallam Co.: Port Angeles, 1914, Foster 2637 (W). Thurston Co. : Gate, 1911, Foster (W). Kitsap Co. : Pt. Orchard, 1890, Parker (W).

This Sticta is rather common and widely distributed in the lowlands, west of the Cascades. Many large and beautiful specimens grow close to the salt water. It is easily determined by the deeply reticulate-pitted thallus and the white sorediate cyphellae. Plate II, B, C, D,

2. Sticta crocata (L.) Ach. Meth. Lich. 277. 1803. Lichen crocatus Linn. Mant. Ait. 310. 1771.

Thallus light brownish green, brown, sepia (R), or Isabella color (R), small to medium sized, membraneous-coriaceous, somewhat wrinkled and 
finally reticulate-ribbed, yellow or greenish yellow soredia scattered, also marginal, often on the ribs, medulla white; below dark brown or blackish, covered with a spongy nap, interspersed with elevated yellow sorediate cyphellae; rather closely attached to the substratum. Apothecia very rare, marginal or scattered, $1.0-2.0 \mathrm{~mm}$. in diameter, sessile; disk dark brown becoming black, flat, thalline margin crenate, finally disappearing; spores brown 1-septate, $16.5-29.7 \times 6.6-8.25 \mu$. This description was made from a U.S.N. Pacific Exploring Expedition specimen, coll. C. Wright, Cape of Good Hope ( $\mathrm{T})$. Tuckerman examined exotic specimens only.

On bark and rocks: Pacific Co.: Long Beach, 1928, 1925b; Chinook, 1940, 2824. San Juan Co.: Waldron Island, Fink (1919).

The yellow soredia on the upper surface, the white medulla, and the sorediate cyphellae, distinguish this species.

3. Sticta fuliginosa (Dicks.) Ach. Meth. Lich. 280. 1803.

Lichen fuliginosus Dicks. Fasc. P1. Crypt. Brit. 1:13. 1785.

Thallus brown, almost black, critrine drab $(R)$, or green-gray $(R)$, small to medium sized, coriaceous-membranaceous, lobes short and rounded, faintly ribbed, beset with dark brown or black isidia, margins slightly wavy to almost entire; below buff or light brown, covered with a nap of short, silky hairs, interspersed with naked, concave, white or pale buff colored cyphellae; loosely attached to the substratum. Sterile in this state. "Apothecia, in a Welch specimen from Mr. Borrer, marginal, smallish, biatoroid, the reddish-brown disk soon convex, and the thin, entire, paler margin disappearing. Spores fusiform, 2-4-locular, soon colorless, 25-46 x 7-9 mic."From (Tuckerman 1882).

On bark and rock: San Juan Co.: San Juan Island, Friday Harbor, 1928, 1849; Pt. Caution, 1928, 2001; Univ. of Wash. Oceano. Lab. trail to Heaven, 1904, 3249; Friday Harbor, 1906, Fink 55 (W). Thurston Co.: Gate, 1911, Foster 1932 (W).

Rather common on rocks near the salt water, the abundant dark colored isidia on the upper surface are a good distinguishing character for this species.

4. Sticta limbata (Sm.) Ach. Meth. Lich. 280. 1803.

Lichen limbatus Sm. in Sm. and Sowerb. Engl. Bot. 16: tab. 1104. 1803.

Thallus drab (R), greenish brown, or almost black, small, orbicular, membranaceous, lobes broad, smooth to slightly pitted, beset especially towards the margins with grayish soredia, margins crenate or undulate, also grayish sorediate; below buff to light brown, covered with soft, fine hairs, interspersed with naked, concave, white, or pale buff-colored cyphellae; loosely attached to the substratum. Sterile in this state.

On bark: San Juan Co.: San Juan Island, Friday Harbor, 1928, 1997. Turn Island, 1928, 1996. Pacific Co.: Chinook, 1940, 2814. Thurston Co.: Gate, 1940, 2965. San Juan Co. : San Juan Island, 1906, Fink 157 (W). 
The small size and the gray soredia make this an easily recognized Sticta.

5. Sticta quercizans Ach. Syn. Lich. 234. 1814.

Lobaria quercizans (Ach.) Michx. Fl. Boreali-Am. 2nd ed., 324. 1820.

Thallus drab $(R)$, cinnamon brown $(R)$, or reddish brown, small to medium sized, cartilagineous-coriaceous, lobes narrow, smooth, densely isidiose on the margins, margins crenate or undulate; below dark brown or almost black, covered with short hairs, interspersed with naked, concave, white, or pale buff-colored cyphellae; rather loosely attached to the substratum. I have seen no fruiting material from the state.

On bark or moss: Clallam Co.: Port Angeles, Coleman's, 1914, Foster 2635 (W). Thurston Co. : Gate, 1911, Foster 1926 (W).

The narrow lobes with isidiose margins make this rather an easy species to recognize; not widely distributed in the state. 


\section{PELTIGERACEAE}

Thallus squamulose or foliose; dorsi-ventral; a well-developed cortex on one or on both surfaces, definite algal and medullary layers; attached to the substratum by rhizoids. Apothecia round or irregular, marginal on either the upper or the lower surface, or scattered on the upper surface, adnate; proper margin present, thalline margin absent; asci 2-8-manyspored; spores hyaline or brown, 1-7-septate. Cephalodia present in some genera.

Alga Palmella, Dactylococcus, or Nostoc.

\section{A. Apothecia marginal.}

B. Apothecia on the upper surface, spores hyaline. 30. Peltigera

B. Apothecia on the lower surface, spores brown or brownish.

29. Nephroma A. Apothecia scattered on the upper surface. 28. Solorina

28. Solorina Ach. Kgl. Vet. Akad. Nya Hand1. 228. 1808.

Thallus foliose, sometimes squamulose, differentiated into a thin or thick pseudoparenchymatous upper cortex and a poorly developed lower cortex, with well-defined algal and medullary layers; loosely attached to the substratum by rhizoids. Apothecia round or irregular, partly immersed to adnate; proper margin thin, concolorous with the thallus, sometimes disappearing, thalline margin absent; hypothecium hyaline; paraphyses septate, unbranched, rather thick; asci 2-8-spored; spores brown, 1-septate.

Alga Palmella or Nostoc.

A. Thallus saffron yellow or reddish orange below.

1. S. crocea

A. Thallus pale gray or brown below.

2. S. saccata

1. Solorina crocea (L.) Ach. Klg. Vet. Akad. Nya Handl. 228. 1808.

Lichen croceus Linn. Sp. P1. 1149. 1753.

Thallus foliose, Madura brown (M.\&P.) or various shades of brown, small to medium sized, orbicular, lobes short and broad, smooth, margins wavy, usually imbricated; below saffron-yellow (M.\&P.) or orange-rufus (M.\&P.) with brownish veins, rhizoids sparse, clustered. Apothecia scattered, 2-10 $\mathrm{mm}$. in diameter, roundish, adnate; disk old Burgundy color 
(M.\&P.), reddish brown, brownish black, or almost black, flat to convex, tumid; asci 8-spored; spores brown, oblong or ellipsoid, 1-septate, 29.7$42.9 \times 9.9-13.2 \mu$.

On soil among mosses: Pierce Co.: Mt. Rainier, near Reflection Lakes, 5000 ft., 1942, 3585. Yakima Co.: Dewey Lake, 4800 ft., 1940. Whatcom Co.: Ruth Mountain, 5500 ft., 1928, L. Sunquist 1865 (W). Olympic Mountains, 5000 ft., Piper 529 (W). Pierce Co.: Mt. Rainier Glacier Basin, 1925, Grant (F).

This species is easily recognized by the bright orange-colored under surface; a beautiful alpine lichen, not common.

2. Solorina saccata (L.) Ach. Klg. Vet. Akad. Nya Handl. 228. 1808.

Lichen saccatus Linn. F1. Suec. 2nd ed., 419. 1755.

Thallus foliose, pearl gray (M.\&P.), Sudan (M.\&P.), dogwood (M.\&P.), yellow beige (M.\&P.), greenish gray, or brown, small to medium sized, thin papery, rosette-like in shape, smooth to granular, sometimes slightly whitish pruinose, lobes irregular in shape, margins entire to slightly crenate; below paler with long concolorous rhizoids. Apothecia scattered, 1.0-5.0 $\mathrm{mm}$. in diameter, immersed; disk dark brown, rose ebony, or almost black, concave; asci 4-spored; spores brown, ellipsoid, 32.0-60.0 x 16.0-27.0 $\mu$.

On soil among mosses and rock: Whatcom Co.: Mt. Baker, $7000 \mathrm{ft}$., Herre (1943).

29. Nephroma Ach. Lich. Univ. 101. 1810.

Thallus foliose, small to medium sized; a well-developed pseudoparenchymatous cortex on both the upper and lower surfaces; also definite algal and medullary layers; often closely attached to the substratum by rhizoids that are well developed or rudimentary. Apothecia circular to reniform, marginal, adnate to the lower surface of the upturned lobes of the thallus; proper margin thin, concolorous with the thallus, often disappearing; thalline margin absent; hypothecium hyaline; paraphyses unbranched, septate; asci 8-spored; hyaline or slightly brownish, 1-3-septate.

Alga Palmella or Nostoc.

A. Under surface tomentose, medulla white, no color change with $\mathrm{KOH}$.

$\mathrm{B}$. With white tubercules below, margins deeply and irregularly wavy.

4. $N$. resupinatum

B. Without white tubercules below, margins fringed with tooth-like lobules. 5. N. resupinatum f. helveticum

A. Under surface smooth to wrinkled.

C. Medulla yellow, turns purple with $\mathrm{KOH}$......

3. N. Iusitanicum

C. Medulla white, no color change with $\mathrm{KOH}$.

D. Lobes sorediate at margins.

2. N. laevigatum f. parile

D. Lobes not sorediate at margins, margins sinuate-crenate.

1. N. laevigatum 
1. Nephroma laevigatum Ach. Syn. Meth. Lich. 242. 1814.

Thallus greenish gray or chestnut-brown, small to medium sized, lobes small and round, smooth to wrinkled and pitted, margins sinuate-crenate; below light or dark brown. Apothecia 1.0-5.0 mm. in diameter, adnate; disk reddish brown or brownish black, flat; proper margin thin, sinuate; spores light brown, fusiform-ellipsoid, or pyriform, 3-septate, $16.5-23.8 \times 6.6-7.0 \mu$. Medulla white, no reaction with $\mathrm{KOH}$.

On bark and mossy rocks: Kitsap Co.: Hidden Ranch near Bremerton, 213 ft., 1931, 1761a. Grays Harbor Co.: Copalis, 1911, Foster 1421 (W).

2. Nephroma laevigatum f. parile (Ach.) Mudd, Manu. Brit. Lich. 81. 1861.

Lichen parilis Ach. Lich. Suec. Prodrom. 164. 1798. Nephroma parile Ach. Lich. Univ. 522. 1810.

Thallus thin, lobes somewhat elongated, margins abundantly gray or brown sorediate; otherwise much like the species.

On bark and mossy rocks: Pend Oreille Co.: Metaline Falls, 2699 ft., 1931, 1476. Clallam Co.: near Port Angeles, 1800 ft., 1914, Foster 2908 (W). Ferry Co.: Republic, 1912, Foster 2358 (W). Incorrectly named Nephroma resupinatum.

3. Nephroma lusitanicum Schaer. Enum. Crit. Lich. Europ. 323. 1850.

Thallus greenish gray, chestnut-brown, small to medium sized, lobes round, smooth and shiny, or slightly wrinkled, margins crenate, crisp, or undulate; below smooth or glabrous, light or dark brown, or almost black, smooth or glabrous. Apothecia 1.0-5.0 mm. in diameter, adnate; disk reddish brown, concave to flat; proper margin thin, sinuate; spores pale brown, fusiform-ellipsoid, 3-septate, 13.0-23.0 x 6.0-7.0 $\mu$. Medulla yellow, purple with $\mathrm{KOH}$.

On bark or rock: Pacific Co. : Loomis Lake, 1940, 2845; Chinook, 1940, 2819. San Juan Co. : San Juan Island, Trout Lake, 1940, 3228; Pt. Caution, 1940, 3275. Kitsap Co.: Hidden Ranch near Bremerton, 213 ft., 1931, 1761a. Thurston Co.: Gate, 1940, 2959. San Juan Co. : Henry Island, 1905, Frye (W) ; Orcas Island, Olga, 1906, Fink 345 (W). Thurston Co.: Gate, 1911, Foster 1938 (W).

Perhaps the commonest Nephroma in the state; the yellow medulla and the purple color change of the medulla with $\mathrm{KOH}$ make this an easily determined species of Nephroma.

4. Nephroma resupinatum (L.) Ach. Lich. Univ. 522. 1810.

Lichen resupinatum Linn. Sp. P1. 1148. 1853. Nephroma tomentosum (Hoffm.) Flot.

Thallus greenish gray, olive-brown, or brown, medium sized to large, lobes round, smooth to slightly tomentose, margin deeply and irregularly 
wavy or laciniate; below lighter in color, densely tomentose, with white tubercules. Apothecia $2.0-15.0 \mathrm{~mm}$. in diameter, adnate; disk reddish brown, tawny when wet, flat becoming uneven; proper margin slightly crenate, concolorous with the thallus; spores pale-brown, fusiform-ellipsoid, 3-septate, 15.8-26.4 x 4.0-6.9 $\mu$. Medulla white, no reaction with $\mathrm{KOH}$.

On bark and rock: Pacific Co.: Loomis Lake, 1940, 2847. King Co.: Grass Mountain Trail, 2200 ft., 1931, 708. Grays Harbor Co.: Westport, 1908, Foster 666 dup. (W). Clallam Co. : Port Angeles, Coleman's, 1914, Foster 2625 (W). Thurston Co.: Gate, 1911, Foster 1947 (W). King Co.: Seattle, 1891, Parker 16 (W).

The white tubercules on the under side are a distinguishing character.

5. Nephroma resupinatum f. helveticum (Ach.) Rabh. Deutschl. Kryptog. F1. 2:68. 1845. Nephroma helveticum Ach. Lichenogr. Univ. 523. 1810.

Thallus gray green, tawny, or brown, small to medium sized, lobes round, smooth, with now and then a few lobules scattered over the surface, margins fringed with tooth-like lobules; below cinnamon-buff $(R)$, smokebrown (M.\&P.), or blackish brown, tomentose. Apothecia 2.0-7.0 mm. in diameter, adnate; disk reddish brown to black, flat; proper margin wavy; spores brownish, fusiform-ellipsoid, 3-septate, 19.8-26.4 x 4.3-6.6 $\mu$. Medulla white, no reaction with $\mathrm{KOH}$.

On bark and rock: Pacific Co.: Chinook, 1940, 2819a. Pierce Co.: White River Camp, 3000 ft., 1928, 1828 ; Mt. Rainier, trail to Seattle Park, 4000 ft., 1940, 3049. Clallam Co.: Port Angeles, Coleman's, 1914, Foster 2626 (W), determined as Nehproma laevigatum. King Co. : Summit, 1907, Foster 646 ( F).

Margins fringed with tooth-like lobules and lack of white tubercules on the under surface separate this form from the species.

\section{Peltigera Willd. F1. Berol. Prodro. 347. 1787.}

Thallus foliose, often large, and wide spreading horizontally, with a well-developed pseudoparenchymatous cortex on the upper surface, entirely lacking on the lower surface, definite algal and medullary layers, with rhizoids arising from the lower hyphae; upper surface naked or covered with a fine down, lower surface with more or less anastomosing veins; more or less firmly attached to the substratum by tufted rhizoids. Apothecia circular to elliptical, marginal, adnate on the upper side of the narrow, extended lobes; proper margin present, thalline margin absent; hypothecium hyaline or brownish; paraphyses unbranched, septate, enlarged at the tips; asci usually 8-spored, rarely only 6-spored; spores hyaline or brownish, often curved, 3-7-septate.

Alga Palmella, Dactylococcus, or Nostoc.

A. Thallus bearing brown to blackish wart-like bodies (cephalodia), thallus turns bright green when moist. 
B. Thallus large, cephalodia borne only on the upper surface.

1. P. aphthosa

B. Thallus small, cephalodia borne only on the veins of the lower surface. 2. P. venosa

A. Cephalodia absent, thallus does not turn bright green when moist.

C. Upper surface downy, or bearing trichomatic hyphae.

D. Lower surface covered with a dense brown to black tomentum.

6. P. malacea

D. Lower surface reticulated with distinct veins.

E. Veins light brown to black, lighter near the margins.

F. Bearing coralloid branches, usually on the margins, lobes narrow.

8. P. praetextata

F. Not bearing coralloid branches, margins crenate, lobes wider.

E. Veins mostly white, sometimes brownish.

9. P. rufescens

G. Thallus very small to medium sized.

$\mathrm{H}$. Bearing masses of soredia on the upper surface, thallus never exceeding $2.5 \mathrm{~cm}$. in the largest diameter. 5 . P. leptoderma

$H$. Never bearing masses of soredia on the upper surface, thallus often larger.

11. P. spuria

G. Thallus medium sized to large.

I. Thallus large, upper surface irregularly pitted.

4. P. canina f. membranacea

I. Thallus smaller, upper surface not minutely pitted.

3. P. canina

C. Upper surface granular, bearing soredia on the margins, veins forming a close tomentum on the under surface. 10. P. scutata

C. Upper surface smooth and shiny, thallus digitately lobed; apothecia borne on narrow digitately clustered lobules, finally revolute.

7. P. polydactyla

1. Peltigera aphthosa (L.) Willd. Fl. Berol. Prodro. 347. 1787. Lichen aphthosus Linn. Sp. Pl. 1148. 1753.

Thallus apple green, gray green, or some shade of brown, bright green when wet, medium sized to large, smooth, usually sprinkled with cephalodia, lobes broad, rounded, margins strongly ascending; below cream color or smoky black, reticulated with almost white or purplish veins, bearing scattered rhizoids. Apothecia on the tips of somewhat elongated lobules, revolute, adnate; disk dark reddish brown, almost black; proper margin, concolorous with the thallus, thin, crenate; asci 8-spored; spores hyaline or brownish, acicular, 3-7-septate, 48.0-75.9 $\times$ 4.9-5.2 $\mu$.

On soil and on moss covered rocks: San Juan Co.: San Juan Island, Friday Harbor, 1928, 2006. Clallam Co.: Lake Sutherland, 590 ft., 1931, 1025. King Co.: Richmond Highlands, 300 ft., 1931, 581. Yakima Co.: Deadhorse hill, $4500 \mathrm{ft} ., 1931$, 983. Chelan Co.: Rainbow Trail, $3800 \mathrm{ft}$, 1931, 1264 Pend Oreille Co.: Metaline Falls, 2600 ft., 1931, 1481. San 
Juan Co.: San Juan Island, Friday Harbor, 1906, Fink 20 (W). Jefferson Co. : Elwha River Valley, 4000 ft., 1907, Frye 51 (W). Clallam Co. : Dungeness, 1913, Foster 2546 (W). Spokane Co.: Spokane, near the Natatorium, 1911, T. A. Bonser (W).

2. Peltigera venosa (L.) Baumg. F1. Lips. 561. 1790. Lichen venosus Linn. Sp. P1. 1148. 1753.

Thallus pale olive-green, ashy, or brownish, bright green when wet, small, fan-shaped, smooth, margins wavy, ascending; below whitish, reticulated with brownish black veins, often spreading together into a close nap, at times cephalodia occur on the veins. Apothecia marginal, $2.0-5.5 \mathrm{~mm}$. in diameter, round, horizontal, adnate; disk reddish brown to almost black, flat; proper margin slightly toothed, soon disappearing; asci 8-spored; spores hyaline or brownish, fusiform, 3-septate, 33.0-42.9 x 6.6-9.2 $\mu$.

On soil: San Juan Co.: Cypress Island, 1928, 1856; Orcas Island, Mt. Constitution, 2500 ft., 1928, 2009. King Co. : Richmond Highlands, 300 ft., 1928, 1855. Yakima Co.: Bumping Lake, 3400 ft., 1931, 933. Okanogan Co.: Twisp Summit, 6066 ft., 1931, 1319. Chelan Co.: Rainbow Trail, $3800 \mathrm{ft} ., 1931,1263$. Stevens Co.: Boyds, 1470 ft., 1931, 1440. Jefferson Co.: Olympic Mountains, near foot of Humes Glacier, $5000 \mathrm{ft}$., 1907, Frye 17 (W). Clallam Co.: Port Angeles, 1914, Foster 2620 (W). Asotin Co.: Blue Mountains, 1929, Jones (W).

A common soil lichen widely distributed over the state, easily recognized by the small fan-shaped thallus that turns bright green when wet.

3. Peltigera canina (L.) Willd. F1. Berol. Prodro. 347. 1787.

Lichen caninus Linn. Sp. P1. 1149. 1753.

Thallus greenish gray, ashy, or various shades of brown, medium sized to large, partially covered with trichomatic hyphae, a hair-like down, especially toward the tips of the lobes, margins irregularly crenate, ascending; below whitish, rarely brown, with veins of same color or darker, with scattered long whitish rhizoids. Apothecia large, 4.0-14.0 mm. across, round, horizontal, or commonly revolute, borne on the tips of extended lobules, that are often suberect, adnate; disk reddish brown to dark brown; proper margin becoming wavy or crenate; asci 8-spored; spores hyaline, fusiform, elongate, 3-7-septate, 46.2-59.4 x 4.0-5.9 $\mu$.

On soil, mosses, or decaying logs: San Juan Co.: Cypress Island, 1928, 1857. Pacific Co.: Nahcotta, 1930, 450. Pierce Co.: Mt. Rainier, Berkeley Park, 6000 ft., 1931, 626. Kittitas Co. : Easton, 2168 ft., 1931, 856. Chelan Co.: Stehekin, 1140 ft., 1931, 1286. Klickitat Co.: Goldendale, 2000 ft., 1931, 1690. Whitman Co.: Tekoa Mountains, 2900 ft., 1931, 1590. San Juan Co.: Brown Island, 1906, Fink 132 (W). Clallam Co.: Dungeness, 1913, Foster 2547 (W). Whatcom Co. : Ruth Mountain, 5500 ft., 1928, Sunquist 1436 (W).

A very common species widely distributed, distinguished by the tricho- 
matic hyphae on the upper surface and a light-colored or rarely brownish lower surface. Plate III, B, C.

4. Peltigera canina f. membranacea (Ach.) Duby, Bot. Gall. 2:598. 1830. Peltidea canina var. membranacea Ach. Lich. Univ. 518. 1810.

Thallus greenish gray, ashy, or various shades of brown, large, thin, pitted, well covered with trichomatic hyphae, hair-like down, lobes broad, rounded, margins wavy; below whitish, rarely brown, reticulated with veins of same color or darker, rhizoids scattered, whitish or brown. Apothecia small, 3.0-5.0 mm. across, otherwise the same as the species.

On soil, mosses, or decaying logs: San Juan Co.: San Juan Island, Pt. Caution, 1928, 2010. King Co.: Edgewood, 300 ft., 1931, 1817; Mt. Rainier, White River Camp, 3900 ft., 1940, 1265. Columbia Co.: Blue Mountains, 3500 ft., 1931, 1622. Clallam Co.: Port Angeles, 1914, Foster (W). Skagit Co. : Hamilton, 1905, Foster (W).

5. Peltigera leptoderma Nyl., Syn. Lich. $1: 325.1850$.

Peltigera canina var. sorediata Tuck.

Peltigera sorediata (Schaer.) Fink.

Thallus pale grayish green or light brown, small, downy, lobes round, with prominent groups of erumpent whitish or gray-green soredia near the center, margins slightly ascending, wavy; below white or ashy, with a close tomentum of fine veins and closely attached to the substratum with rather long pale rhizoids. Apothecia rare, sterile in the state.

On soil or rock: Yakima Co.: Bumping Lake, 3400 ft., 1931, 937; American River Camp, 2800 ft., 1931, 953. Chelan Co.: Rainbow Trail, 3800 ft., 1931, 1265. Ferry Co.: Laurier, 1644 ft., 1931, 1444. San Juan Co. : Orcas Island, Olga, 1906, Fink 346 (W). Snohomish Co. : Marysville, 1926, Grant (F).

This Peltigera is rather easily recognized by the small thallus, with scattered gray-green or whitish soredial masses.

6. Peltigera malacea (Ach.) Funck, Crypt. Gewach. Heft 33:5. 1827. Peltidea malacea Ach. Syn. 240. 1814.

Thallus buffy-olive $(R)$, tawny-olive $(R)$, or various shades of brown, medium sized, slightly pulverulent or downy, rather thick and spongy, horizontal or wavy; below brown or black with a close tomentum, paler toward the edges, rhizoids scarce. Apothecia 3.0-7.0 mm. across, round, revolute, borne on the tips of extended lobules, adnate; disk reddish brown or black; proper margin crenulate; asci 8-spored, spores hyaline, acicular, 3-5-septate, 22.9-75.9 x 2.6-5.0 $\mu$.

On soil and rock: Island Co.: Whidbey Island, 1931, 1771. Pierce Co. : Berkeley Park, 6000 ft., 1940, 3023. Yakima Co. : Deadhorse Hill, 4500 ft., 1931, 984. Chelan Co.: Rainbow Trail, 3500 ft., 1931, 1281; Merritt, 2186 ft., 1931, 1079. Ferry Co. : Laurier, 1644 ft., 1931, 1431. 
No fruiting specimens seen by the writer from this state as yet, but it can easily be determined by the dense dark-colored tomentum on the lower surface.

7. Peltigera polydactyla (Neck.) Hoffm. Descript. and Adumbr. P1. Lich. $1: 19.1790$.

Lichen polydactylus Neck. Meth. Musc. 85. 1771.

Thallus greenish glaucescent $(\mathrm{R})$, grayish green or brownish, medium sized to large, thin, smooth, and shiny, lobes digitate, often ascending; below white or brownish, reticulate, with wide, whitish or brownish to blackish veins; closely attached to the substratum with brown or black rhizoids. Apothecia 2.0-6.0 mm. across, round or oblong, revolute, borne on the tips of narrowly digitately clustered lobules, adnate; disk reddish brown; proper margin crenulate; asci 8-spored, spores hyaline, acicular, 3-7-septate, $60.0-89.0 \times 2.5-4.0 \mu$.

On soil or mossy tree trunks: Pacific Co.: Loomis Lake, 1931, 777. Clallam Co.: Lake Sutherland, 590 ft., 1931, 1013; West Elwha Trail, 300 ft., 1940, 2896. Kitsap Co. : Hidden Ranch near Bremerton, 213 ft., 1931, 1732. King Co.: Grass Mountain Trail, 2200 ft., 1931, 706.

Distinguished by the smooth and shiny upper surface and by the ascending incurving, digitately clustered fertile lobules.

8. Peltigera praetextata (Flk.) Wainio, Term. Füz., 22:306. 1899.

Peltidea ulorrhiza var. praetextata Flk., in Somrft. Suppl. Fl. Lapp. 123. 1826.

Thallus grayish green, rusty brown, or brown, small to medium sized, slightly downy, lobes narrow, wavy, ascending at the margins, often covered with light or dark coralloid branches, especially toward the margins; below whitish, rarely light brown, reticulated with light to dark brown veins, rhizoids brown not abundant. Apothecia 2.0-5.0 mm. across, round or somewhat oblong, revolute, borne on narrow upright lobules, adnate; disk reddish brown; proper margin denticulate; asci 8 -spored, spores hyaline, acicular, slightly curved, 3-5-septate, 39.6-49.5 x 3.3-4.0 $\mu$.

On soil or decaying logs: San Juan Co. : San Juan Island, Roche Harbor, 1928, 1851. Clallam Co.: Lake Sutherland, 590 ft., 1931, 1034. Kitsap Co.: Hidden Ranch near Bremerton, 213 ft., 1931, 1733. King Co. : Edgewood, 1931, $300 \mathrm{ft} ., 1808$. Pierce Co. : Mt. Rainier, trail to Moraine Park, 3300 ft., 1940, 2991; Dewey Lake, 4800 ft., 1940, 2774. Spokane Co.: Indian Canyon, 1934, Sister Marion Loretta (WTU).

Distinguished by the abundant coralloid branches, scattered over the upper surface and margins of the thallus.

9. Peltigera rufescens (Weis.) Humb., Fl. Frib. Specim. 2. 1793.

Lichen caninus, rufescens Weis. P1. Crypt. Fl. Goett. 79. 1770.

Thallus grayish green or reddish brown, small to medium sized, thick, smooth or downy, lobes narrow, margins crenate, crisp, often upright; 
below whitish, reticulated with very thick, light or dark brown veins, rhizoids of same color. Apothecia $3.0-7.0 \mathrm{~mm}$. across, round or oblong, revolute, borne on the tips of narrow upright lobules, adnate; disk brown, reddish brown, or blackish brown; proper margin denticulate; asci 8-spored; spores hyaline, acicular, straight to slightly curved, 3-7-septate, 42.9-49.5 x 3.3-4.0 $\mu$.

On soil, rock, and decaying logs: Kitsap Co.: Hidden Ranch near Bremerton, 213 ft., 1931, 1727. Pierce Co.: Mt. Rainier, White River Camp, 3000 ft., 1931, 1853 ; Berkeley Park, 6000 ft., 1931, 627. Chelan Co. : Stehekin, 1140 ft., 1931, 1287. Whitman Co.: Tekoa Mountains, 2900 ft., 1931, 1589. King Co.: Seattle, 1891, A. M. Parker 53 (W).

No very good distinguishing characters, the crisped edges help to distinguish it from $P$. canina, which species it resembles rather closely.

10. Peltigera scutata (Dicks.) Duby, Bot. Gall. 2:599. 1830.

Lichen scutatus Dicks., Fasc. P1. Crypt. Brit. 3:18. 1793.

Thallus grayish green or pale tawny brown, small, granular or scurfy, lobes narrow, margins ascending, crenate to wavy, often well covered with grayish green soredia, often involute; below whitish or brown veins forming close tomentum, always paler towards the margins, rhizoids pale at the margins, dark brown near the center, very bushy. Apothecia small, 2.0-4.0 $\mathrm{mm}$. across, round, sometimes revolute, borne on the tips of short lobules, usually erect, adnate; disk reddish or blackish brown; proper margin crenate; asci 8-spored; spores hyaline, acicular, 3-7-septate, 44.0-66.0 x 3.0-5.0 $\mu$.

On soil, decaying logs, trees or rarely on mossy rock: San Juan Co.: San Juan Island, Pt. Caution, 1940, 3268; Orcas Island, Mt. Constitution, 2500 ft., 1928, 2012. Pacific Co. : Long Beach, 1928. Thurston Co.: Grand Mound, 1940, 2949. Kitsap Co.: Hidden Ranch near Bremerton, 213 ft., 1931, 1731. Pierce Co. : Mt. Rainier, Moraine Trail, 3300 ft., 1940, 2997. Kittitas Co.: Roslyn, 2800 ft., 1931, 899a. Klickitat Co.: Goldendale, 2000 ft., 1931, 1699. Clallam Co.: Port Angeles, Birds, 1914, Foster 2621 (W). Thurston Co.: Gate, 1912, Foster 1942 (W). Spokane Co.: Spokane, 1929 ft., 1940, 2750. King Co.: Ronald's Bog, 1934, Sister Marion Loretta (WTU).

This species can be distinguished by the abundant grayish green, sorediate margins.

11. Peltigera spuria (Ach.) DC., in Lam. and DC. Fl. Franc. 3rd ed., 1805.

Lichen spurius Ach. Suec. Prodro. 159. 1798. Peltigera canina var. spuria (Ach.) Schaer.

Thallus pale greenish gray, reddish brown, or various shades of brown, small, when dry stiff, and brittle, partially covered with trichomatic hyphae, margins almost entire; below whitish, reticulated with white or brown 
veins, rhizoids scarce. Apothecia 3.0-5.0 mm. across, round to oblong, revolute, borne on the tips of short digitately clustered lobules, adnate; disk reddish brown; proper margin irregularly crenulate; asci 8-spored; spores hyaline, acicular, slightly curved, 3-7-septate, 22.9-75.9 x 2.6-5.0 $\mu$.

On soil and mossy rocks: Island Co.: Whidbey Island, 1931, 1776. Mason Co.: Shelton, 1931, 1225. Kitsap Co. : Hidden Ranch near Bremerton, 213 ft., 1930, 1854. Pierce Co.: Mt. Rainier, trail to Mowich Lake, 3500 ft., 1940, 3062. San Juan Co.: Orcas Island, Olga, 1906, Fink 347 (W).

This species resembles $P$. venosa somewhat in the small size and shape of the thallus, but the revolute apothecia easily separate it from that species.

\section{LECIDEACEAE}

Thallus crustose or squamulose, sometimes marginally lobed, differentiated into definite cortical, algal, and medulla layers, or in some genera showing little differentiation; attached to the substratum by hyphal rhizoids. Apothecia circular, scattered, adnate, sessile or rarely stipitate ; proper margin well developed, thalline margin absent ; asci usually 8-spored, sometimes 16-30-spored; spores hyaline or brown, nonseptate, 2-many-septate, or muriform.

Alga Protococcus or Gloeocapsa.

A. Spores nonseptate.

B. Spores very large, thick walled.

32. Mycoblastus

$B$. Spores not very large, thin walled. 31. Lecidea A. Spores septate.

C. Spores 1-many transversely septate.

D. Spores 1-septate.

33. Catillaria

D. Spores 2-many-septate.

E. Thallus non-corticate, crustose.

34. Bacidia

E. Thallus corticate, squamulose.

35. Toninia

C. Spores muriform, rarely only transversely septate.

F. Spores hyaline.

36. Lopadium

F. Spores normally brown, rarely hyaline......

37. Rhizocarpon

\section{Lecidea Ach. Meth. Lich. 32. 1803.}

Thallus crustose, granulose, areolate, or squamulose, often lobate at the margin, sometimes bearing soredia or cephalodia, with a typical pseudoparenchymous upper cortex or one of closely packed hyphae, distinct algal and medullary layers, and a lower cortex absent or poorly developed; in some genera there is no differentiation into definite layers. Apothecia usually circular, sometimes angulate, adnate or sessile; proper margin concolorous with the disk, often disappearing; hypothecium hyaline, brown, or almost black; paraphyses rarely branched, sometimes forked near the apex, the amount of coherence is variable; asci usually 8-spored, rarely 16-spored; 
spores hyaline, nonseptate. A large and difficult genus.

Alga Protococcus.

A. Usually on rock, rarely on soil.

B. Thallus subfruticose.

17. L. Pringlei

B. Thallus squamulose.

C. Squamules white edged.

34. L. rubiformis

D. Squamules ample, margins ascendant, white below with a few rhizoids.

35. L. Russellii

D. Squamules not so large, margins not noticeably ascendant, white below without rhizoids.

C. Squamules black edged.

33. L. ostreata

C. Squamules brown edged, entire upper surface brown. 32. L. lurida

B. Thallus squamulose-areolate, edges raised, grayish black.

E. Black hypothallus prominent, marginal areoles not elongated.

11. L. fuscoatra

E. Black hypothallus not prominent, marginal areoles elongated.

5. L. atrobrunnea

B. Thallus areolate or granulose.

F. Thallus some shade of yellow, orange, or red.

G. Hypothecium light-colored, often hyaline.

4. L. armeniaca

G. Hypothecium dark-colored, brown or black.

$\mathrm{H}$. Limiting black hypothallus present; medulla not blue with IKI.

2. L. albocaerulescens var. flavocaerulescens

H. Limiting black hypothallus present; medulla blue with IKI.

13. L. lapicida var. oxydata

F. Thallus never decidedly yellow, orange, or red.

I. Hypothecium light-colored, often hyaline.

9. L. cyanea

I. Hypothecium dark-colored, brown or black.

J. Disk always conspicuously pruinose........1. L. albocaerulescens

J. Disk never or only slightly pruinose.

$\mathrm{K}$. Thallus usually absent, spores poorly developed.

7. L. auriculata var. diducens

$\mathrm{K}$. Thallus usually present, spores well developed, rarely less than $10 \mu$ long.

L. Tips of paraphyses bluish black.

M. Hypothecium always dark brown.

N. Paraphyses free 15. L. parasema

N. Paraphyses coherent.

18. L. vorticosa

M. Hypothecium hyaline or yellow-brown.

$O$. Thallus yellow with $\mathrm{KOH}$, faint red with $\mathrm{CaOCl}_{2}$.

14. L. latypea

O. Thallus not yellow with $\mathrm{KOH}$, but red with $\mathrm{CaOCl}_{2}$. 16. L. parasema var. theioplaca

L. Tips of paraphyses not bluish black.

$P$. Thallus very thick, medulla blue with IKI.

12. L. lapicidia 
P. Thallus not very thick, medulla not blue with IKI.

Q. Spores rarely less than $15 \mu$ long........ 8. L. contigua

Q. Spores rarely more than $15 \mu$ long......6. L. auriculata

A. On bark or old wood.

B. Bordered by conspicuous black hypothalline lines.

C. Hypothecium yellowish brown or dark brown.

C. Hypothecium hyaline.

10. L. elaeochroma f. geographica

28. L. varians

B. Not bordered by conspicuous black hypothalline lines.

D. Hypothecium rarely hyaline, usually brown, disk of apothecium black.

E. Paraphyses free, tips bluish black.

15. L. parasema

E. Paraphyses coherent, tips colorless.

33. L. ostreata

D. Hypothecium hyaline, disk variously colored.

F. Disk scarlet.

20. L. cinnabarina

F. Disk not scarlet.

G. Spores broadly ellipsoid, thick walled.

27. L. tornoensis

$\mathrm{G}$. Spores not broadly ellipsoid, thin walled.

$\mathrm{H}$. Always on bark.

21. L. furvonigrans

$\mathrm{H}$. Not always on bark.

I. Thallus absent or very thin and inconspicuous.

J. Margin of apothecia not darker than the disk.

19. L. carmulenta

J. Margin of apothecia not darker than the disk.

I. Thallus present, consisting of granules.

24. L. myriocarpella

$\mathrm{K}$. Thallus brown or olive brown, margin of apothecia becoming lobate.

23. L. holopolia

$\mathrm{K}$. Thallus yellowish white or greenish gray, margin of apothecia flexuous

25. L. paddensis

A. Usually on soil or moss, rarely on old wood.

B. Thallus granulose.

C. Apothecia hemispherical or globose, hypothecium brown.

C. Apothecia circular, hypothecium pale yellow.

3. L. arctica

B. Thallus squamulose (Psora).

D. Thallus flesh-color ( $R$ ), an orange shade, or brick red.

D. Thallus some shade of gray or brown.

E. Squamules white edged.

29. L. decipiens

E. Squamules not white edged.

F. Light colored under surface of squamules easily seen, showy, prominent. 31. L. globifera

F. Under surface of squamules not easily seen.

G. Hypothecium hyaline or pale yellow. 30. L. demissa

G. Hypothecium dark brown. 32. L. lurida 
1. Lecidea albocaerulescens (Wulf.) Ach. Meth. Lich. 52. 1803.

Lichen albocaerulescens Wulf. in Jaqu. Coll. Bot. 2:184. 1788.

Thallus gray or greenish gray, thin to moderately thick, minutely rimose-areolate. Apothecia circular or slightly irregular, $0.5-1.2 \mathrm{~mm}$. in diameter, immersed or adnate; disk black, usually white pruinose, flat; proper margin thick, black, rarely disappearing; hypothecium thick, dark brown or black; paraphyses coherent, tips enlarged slightly, colored; asci 8-spored; spores hyaline, ellipsoid, nonseptate, 14.0-22.8 x 7.6-9.5 $\mu$.

On rocks: Clallam Co. : Elwha River Bridge, 1911, Foster 1792 (F).

2. Lecidea albocaerulescens var. flavocaerulescens (Hornem.) Schaer. Lich. Helvet. Spicil. sect. $3: 143,1828$.

Lecidea flavocaerulescens Hornem. Icon. P1. Dan. 8: fasc. 24, 5. 1810.

Thallus reddish orange, with a limiting black hypothallus; disk naked or very slightly pruinose; otherwise like the species.

On rock: Whatcom Co.: Mt. Baker, Galena, 3800 ft., 1931, 1200. Yakima Co.: Dewey Lake, 4800 ft., 1940, 3359. Clallam Co.: Lake Crescent, Storm King Mountain, 4300 ft., 1935; A. H. Smith 2267 (F.)

This lichen is easily recognized by the reddish orange of the thallus and the black limiting hypothallus.

3. Lecidea arctica Somrft. Suppl. F1. Lapp. 156. 1826.

Thallus brownish gray, granulose, granules minute, globular, wide spread and finally heaped. Apothecia hemispherical to globose, often irregular, sometimes conglomerate, $0.2-0.8 \mathrm{~mm}$. in diameter, immersed to adnate; disk very black, faintly gray pruinose; proper margin soon disappearing; hypothecium brown; paraphyses coherent; asci 8-spored; spores hyaline, oblong-ellipsoid, nonseptate, 15-25 x 6-8 $\mu$.

On moss or soil: Washington Territory, Cascade Mountains, 1883, Brandegee 50 (T). Whatcom Co.: Mt. Baker, 6000 to $7000 \mathrm{ft}$., Herre (1919).

4. Lecidea armeniaca (Lam. and DC.) Fries Syst. Orb. Veg. pars 1, 286. 1825.

Rhizocarpon armeniacum Lam. and DC. F1. Franc. 3rd ed., 2:366. 1805.

Thallus yellow, pale yellowish orange with a copperish tinge, or bay $(\mathrm{R})$, areoles thick, scattered or crowded, turgid and plicate-rugose, black hypothallus showing between areoles also forming a fringe around the circumference. Apothecia circular, 0.5-2.0 mm. in diameter, immersed; disk black, naked, flat to convex; proper margin soon disappearing; hypotheciun pale yellow or brownish; paraphyses coherent, tips colored; asci 8-spored, spores hyaline; ellipsoid, nonseptate, 9-13 x 5-6 $\mu$.

On rock: Washington Territory, Cascade Mountains, Brandegee (T). 
5. Lecidea atrobrunnea (Ram.) Schaer. Lich. Helvet. Spicil. 134. 1828. Lichen atrobrunneus Ram. in Lam. and DC. Fl. Franc. 3rd ed., 2:367. 1805.

Thallus chestnut-brown or reddish brown, squamulose-areolate, areoles smooth and shiny, turgid, tough, flat to convex, lobed, scattered or imbricated, edges wavy and ascending, marginal areoles often elongated. Apothecia circular, $0.5-1.0 \mathrm{~mm}$. in diameter, sessile; disk black, concave to slightly convex; proper margin black, thick, prominent, becoming flexuose; hypothecium brown; paraphyses coherent, tips colored; asci 8-spored; spores hyaline, ellipsoid, nonseptate, 9.9-12.0 x 3.0-4.3 $\mu$.

On rock: Yakima Co.: gulch near Naches, 1350 ft., 1931, 903. Washington Territory, Brandegee 48 (F).

6. Lecidea auriculata Th. Fr. Nov. Act. Reg. Soc. Sci. Upsal. ser. 3, $3: 313.1861$.

Thallus grayish white or pale brown, rimose-areolate, thin, rough. Apothecia circular, at length variously lobed and irregular, $0.3-1.0 \mathrm{~mm}$. in diameter, adnate; disk black, flat to convex; proper margin concolorous with the disk somewhat raised, finally disappearing; hypothecium pale brown or olive brown; paraphyses coherent, tips darkened; asci 8-spored; spores hyaline, oblong to ellipsoid, nonseptate, 7.0-11.4 x 3.8-4.0 $\mu$.

On rock: Columbia Co.: Blue Mountains, 5000 ft., 1931, 1627.

7. Lecidea auriculata var. diducens (Nyl.) Th. Fr. Lich. Scand. 499. 1874. Lecidea diducens Nyl. Flora $48: 148.1865$.

Thallus absent. Apothecia in scattered crowded groups, circular to irregular perhaps due to crowding; spores small and poorly developed.

On rock: Chelan Co.: Beaver Summit, 2800 ft., 1931, 1340; butte near Chelan, 2000 ft., 1931, 1355. Okanogan Co. : near Tonasket, 2000 ft., 1931, 1385.

8. Lecidea contigua (Sm.) Fries Nov. Sched. Critic. 14. 1827.

Lichen contiguus Sm. in Sm. and Sowerb. Engl. Bot. 12; tab. 821. 1801.

Thallus gray, grayish white, or greenish gray, rough and warty, becoming rimose-areolate, irregularly spread over the substratum, sometimes thin and scattered. Apothecia circular, 0.4-1.3 mm. in diameter, immersed to adnate; disk black, flat to convex, rarely faintly pruinose; proper margin black, thin, raised, finally disappearing; hypothecium thick, dark brown; paraphyses slender, subcoherent, dark or olivaceous brown at the tips; asci 8-spored; spores hyaline, rather large, ellipsoid, 13.2-23.1 x 6.0-9.0 $\mu$.

On rock: Whatcom Co.: Ruth Mountain, 6800 ft., 1928, 1946. Pierce Co.: Mt. Rainier, Paradise Valley, 5500 ft., 1928, 1939; Berkeley Park, $6000 \mathrm{ft} ., 1940$, 3028. Ferry Co.: Mt. Gibraltar, 3100 ft., 1940, 3153. Washington Territory, Suksdorf 288 (S). 
9. Lecidea cyanea (Ach.) Röhl. Deutschl. Fl. 2 Abt. 3 :32. 1813.

Lecidea lapicida var. cyanea Ach. Meth. Lich. 38. 1803.

Lecidea tessellata (Ach.) Flk.

Thallus gray or greenish gray, rimose-areolate in round to angular areoles on a more or less prominent black hypothallus. Apothecia circular to slightly angular, $0.5-2.0 \mathrm{~mm}$. in diameter, immersed to adnate; disk black and horny, at first pruinose, flat to convex; proper margin thin elevated, persistent, finally flexuous; hypothecium hyaline; paraphyses coherent, tips brownish black; asci 8-spored; spores hyaline, ellipsoid, nonseptate, 6-8.5 x 4.0-6.0 $\mu$.

On rock: Whatcom Co.: Ruth Mountain, 6800 ft., 1928, Sunquist 1877. Yakima Co.: gulch near Naches, 1350 ft., 1931, 906; 1940, 3174. Chelan Co.: butte near Chelan, 2000 ft., 1931, 1376. Okanogan Co.: gulch near Tonasket, 1200 ft., 1931, 1379. Columbia Co.: New Hope, 1500 ft., 1931, 1565. Garfield Co. : Pomeroy, $1860 \mathrm{ft} ., 1931,1571$.

The black apothecia on the gray thallus make this Lecidea resemble a mosaic pavement when examined under a hand lens.

10. Lecidea elaeochroma f. geographica (Bagl.) Zahlbr. Cat. Lich. Univers. $3: 571.1925$.

Lecidea enterolenca var. geographica Bagl. in Memor. della Royal Acad. Sci. Torino, ser. $2,17: 421.1857$.

Thallus dark olive-buff $(R)$ or citrine-drab $(R)$, small patches of minute crumb-like granules scattered on a black hypothallus and bordered by a conspicuous black hypothalline line. Apothecia circular, $0.2-0.8 \mathrm{~mm}$. in diameter, immersed to adnate; disk black, concave to flat; proper margin thin, high, black, finally disappearing; hypothecium dark yellowish brown; paraphyses free, slightly enlarged at the tips; asci 8-spored; spores hyaline, ellipsoid, nonseptate, $13.2-15.0 \times 7.0-8.0 \mu$.

On bark: Pacific Co.: Chinook, 1931, 792. Clallam Co.: Elwha River valley, near Port Angeles, 1940, 2898. Mason Co.: Shelton, 1931, 1217.

This is an easily determined bark lichen because of the conspicuous black hypothalline lines and dark hypothecium.

11. Lecidea fuscoatra (L.) Ach. Meth. Lich. 44. 1803.

Lichen fuscoater Linn. Sp. Pl. 1140. 1753.

Thallus brown, reddish brown, blackish brown, squamulose-areolate, areoles thin, round, lobulate, angulate, flat, sometimes slightly concave or convex, edges blackish gray, usually slightly raised, scattered or rather closely crowded, black hypothallus often very distinct. Apothecia circular, sometimes angular, $0.5-1.5 \mathrm{~mm}$. in diameter, adnate; disk black, slightly pruinose at first, becoming naked, flat to convex; proper margin black, thin, finally disappearing; hypothecium pale brown or dark brown; paraphyses coherent, tips dark colored; asci 8-spored; spores hyaline, ellipsoid, nonseptate, $9.9-13.2 \times 5.0-6.0 \mu$. 
On rock: Pierce Co.: White River Camp, 3000 ft., 1928, 1942; Mt. Rainier, Summerland Park, $6500 \mathrm{ft} ., 1117 a$. Okanogan Co.: gulch near Tonasket, 1200 ft., 1931, 1366. Spokane Co.: Mt. Spokane, 5808 ft., 1931, 1497. Columbia Co.: Blue Mountains, 5000 ft., 1931, 1627a. Clallam Co.: Lake Crescent, Storm King Mountain, $3500 \mathrm{ft} .$, A. H. Smith 2237 (F). Klickitat Co.: Goldendale, 1909, Foster 1187 (F). Washington Territory, Suksdorf 9 (T).

This lichen is abundant and widely distributed in the state.

\section{Lecidea lapicida Ach. Meth. Lich. 37. 1803.}

Lichen lapicida Ach. Lichenogr. Suec. Prodro. 61. 1798.

Thallus gray, greenish gray, or brownish gray, thick areolate, areoles contiguous, flat to slightly convex, angulate. Apothecia circular, 0.4-2.0 mm. in diameter, partly immersed to adnate; disk black, naked, flat becoming convex; proper margin brownish black, thin, undulate, persistent; hypothecium brown; paraphyses subcoherent, tips brownish thickened; asci 8-spored; spores hyaline, ellipsoid, nonseptate, 11.0-13.2 x 5.9-7.0 $\mu$. Medulla violet then blue with IKI.

On rock: Whatcom Co.: Ruth Mountain, 6800 ft., 1928, L. Sunquist 1946. Pierce Co.: Mt. Rainier, Yakima Park, 6300 ft., 592. Olympic Mountains, Boulder Peak, 6000 ft., 1935, A. H. Smith 2736 (F).

13. Lecidea lapicida var. oxydata Rabh. Deutschl. Kryplog.-Fl. $2: 84.1845$.

Thallus tawny $(\mathrm{R})$ or dull orange red, usually thinner than the species; otherwise like the species. Medulla violet then blue with IKI.

On rock: San Juan Co.: Orcas Island, Mt. Constitution, 2500 ft., 1928, 2014.

The orange red color of the thallus, plus the blue color reaction of the medulla with IKI makes this an easy Lecidea to identify.

14. Lecidea latypea Ach. Meth. Lich. Suppl. 10. 1803.

Lecidea sabuletorum Fries Nov. Sched. Critic. 8. 1826.

Thallus whitish gray or yellowish white, moderately thick, verrucoseareolate, verrucae contiguous or scattered, flat or becoming subglobose, margins crenulate, at times the thallus becomes granulose. Apothecia circular, 0.4-1.2 mm. in diameter, partly immersed to adnate; disk black, flat to depressed, becoming convex at times; proper margin concolorous or brownish black, thick at first, becoming thin, usually persisting ; hypothecium yellowish brown or brown; paraphyses coherent to free, tips swollen, bluish black; asci 8-spored; spores hyaline, broadly ellipsoid, nonseptate, 11.5-16.4 x 6.2-9.9 $\mu$. Thallus yellow with $\mathrm{KOH}$, faint red with $\mathrm{CaOCl}_{2}$.

On rock: San Juan Co.: Turn Island, 1928, 2013. Yakima Co. : Deadhorse Hill, 4000 ft., 1931, 959. San Juan Co.: Brown Island, 1906, Fink $127(\mathrm{~F})$. 


\title{
15. Lecidea parasema Ach. Meth. Lich. 35. 1803.
}

\author{
Lichen parasemus Ach. Lich. Suec. Prodro. 64. 1798. \\ Lecidea enteroleuca Ach.
}

Thallus gray, dark olive-buff $(R)$, greenish gray, or yellowish gray, thin and rather smooth, contiguous, becoming areolate, areoles minute, warty, sometimes granulose. Apothecia circular, 0.5-1.5 mm. in diameter, adnate; disk black, flat to somewhat convex; proper margin black, prominent, entire, becoming flexuous, finally disappearing; hypothecium rarely hyaline, usually brown; paraphyses free, with enlarged bluish black tips; asci 8-spored; spores hyaline, ovoid-ellipsoid, nonseptate, 8.0-16.0 x 6.6-8.3 $\mu$.

On wood or bark, rarely on rock: San Juan Co.: San Juan Island, Pt. Caution, 1928, 2015. Pacific Co.: Chinook, 1940, 2810. Kittitas Co.: Easton, 2200 ft., 1931, 843. Klickitat Co.: Goldendale, 2000 ft., 1931, 1685. Whitman Co.: Tekoa Mountains, 2900 ft., 1931, 1600a. Mason Co.: Hoodsport, 1912, Foster 2110 (F).

16. Lecidea parasema var. theioplaca (Tuck.) Zahlbr. Cat. Lich. Univers. $3: 664.1925$.

Lecidea enterolenca var. theioplaca Tuck. Gen. Lich. 179. 1872.

Thallus pale yellowish, verrucose, thick; hypothecium always brown. Thallus not yellow with $\mathrm{KOH}$, but red with $\mathrm{CaOCl}_{2}$; otherwise like the species.

On rock: San Juan Co.: Shaw Island, 1928, 1944. Washington Territory, Cascade Mountains, 1883, Brandegee 104 (F).

17. Lecidea Pringlei Tuck. Bull. Torr. Bot. Club, $10: 22.1883$.

Thallus dark green or black, fruticose, consisting of crowded erect branched trunks, extending into slender naked irregularly branched stems dilated at the tips, often becoming densely rough and folded, passing into root-like branchlets at the base. Apothecia circular, 1.0-5.0 mm. in diameter; disk reddish brown or black, flat, soon becoming wavy, lobed and wrinkled; proper margin brown or black, thick, shiny, irregular, and finally disappearing; asci 8-spored; spores hyaline, oblong-ellipsoid, nonseptate but sometimes falsely 1 -septate, $9.0-10.0 \times 3.0-5.0 \mu$.

On rock: Washington Territory, Cascade Mountains, eastern slope, Brandegee 34, cotype (T).

$A$ rare and most unusual Lecidea.

18. Lecidea vorticosa (Flk.) Körb. Syst. Lich. Ger. 251. 1855.

Lecidea sabuletorum var. vorticosa Flk. Gesell. Naturf. Freund Berlin, Mag. 2 :311. 1808.

Thallus grayish white or gray, granulose, thin, granules crowded, heaped or dispersed, sometimes entirely absent. Apothecia circular or irregular, often closely crowded and confluent, $0.5-1.8 \mathrm{~mm}$. in diameter, 
sessile; disk black, velvety, usually flat, sometimes concave or convex; proper margin black, prominent, raised, crenulate, flexuous or lobed, rarely disappearing; hypothecium dark brown; paraphyses coherent, tips bluish black; asci 8-spored; spores hyaline, ellipsoid, 9.9-13.2 x 3.3-4.0

On rock: Kittitas Co.: Roslyn, 2800 ft., 1931, 897. Chelan Co.: Zena, 800 ft., 1931, 1107a. Spokane Co.: Mt. Spokane, 5808 ft., 1931, 1496.

19. Lecidea carnulenta (Tuck.) Fink, Con. U.S. Nat. Herb. 14:73. 1910. Biatora carmulento Tuck. Proc. Am. Acad. Arts and Sci. 12:179. 1877.

Thallus usually absent, when present grayish green, consisting of a very thin scattered crust. Apothecia circular, 0.2-0.8 mm. in diameter, adnate; disk pale yellow, pinkish brown, or reddish brown, rather strongly convex; proper margin often darker in color than the disk, disappearing early; hypothecium hyaline or yellowish brown; paraphyses gelatinized, tips sometimes brown; asci 8-spored; spores hyaline, ellipsoid, nonseptate, 7.0-10.5 x 3.0-5.0 $\mu$.

On old wood or bark: King-Kittitas Co.: Snoqualmie Pass, 3010 ft., 1931, 808. Clallam Co.: Olympic Mountains, Olympic Hot Springs, 1935, A. H. Smith 1990 (F).

The distinguishing character is the darker colored proper margin.

20. Lecidea cinnabarina Somrft. Kgl. Vet. Akad. Handl. 114. 1823.

Biatora cinnabarina (Somrft.) Fries.

Thallus white or greenish gray, effuse, thin, granulate, rough and warty, sometimes sorediate. Apothecia circular, sometimes flexuous-lobate, rarely proliferous, 9.2-8.0 mm. in diameter, adnate; disk scarlet, flat to convex; proper margin lighter colored and soon disappearing; hypothecium hyaline; paraphyses coherent; asci 8 -spored; spores hyaline oblong, nonseptate, $6.6-12.0 \times 2.5-3.3 \mu$.

On bark: Pierce Co.: White River Camp, 3000 ft., 1928, 1984 and 1940, 3106. Pierce-Yakima Co.: Chinook Pass, 5440 ft., 1931, 1558. Clallam Co.: Crystal Springs above Olympic Hot Springs, 1914, Foster 2871 (W). Trail to top of Mt. Elinor, 3500-4500 ft., Foster 2107 (F). Washington Territory, Suksdorf (F).

The scarlet disk makes this a very distinctive bark Lecidea.

21. Lecidea furvonigrans (Tuck.) Zahlbr. Cat. Lich. Univers. 3 :762. 1925. Biatora furvonigrans Tuck. Syn. N. Am. Lich. 2:129. 1888.

Thallus brown, thin, wide spread, smooth or rough and verrucose, on a black hypothallus. Apothecia circular, minute, 0.2-0.4 mm. in diameter, sessile; disk brownish black or black, convex; proper margin diappearing very early; hypothecium hyaline; paraphyses conglutinate, tips brownish; asci 8-spored; spores hyaline, oblong, nonseptate, 13.0-15.2 × 3.0-6.0 $\mu$.

On bark: Pacific Co.: along Naselle River, 1940, 2939b. Yakima Co.: 
Dewey Lake, 4800 ft., 1940, 2786. Washington Territory, 1882, Suksdorf 209, Type specimen (T).

22. Lecidea granulosa (Ehrh.) Ach. Meth. Lich. 65. 1803.

Lichen granulosus Ehrh. Pt. Crypt. ex. No. 145, 1785.

Biatora granulosa (Ehrh.) Flot.

Thallus of grayish white, yellowish white, or greenish gray granules, crowded and imbricated, hemispherical, often forming a rather thick, sorediate crust, or squamulose, often lobate at the circumference. Apothecia circular, clustered, often confluent and irregular in shape, $0.5-2.0 \mathrm{~mm}$. in diameter, adnate; disk flesh colored $(\mathrm{R})$, olive brown, or black, dull, sometimes papillate, flat to convex; proper margin often paler than the disk, raised, disappearing soon; hypothecium pale yellow; paraphyses coherent, tips brownish; asci 8-spored; spores hyaline, ovoid-ellipsoid, nonseptate, 9.9-16.5 x 4.0-5.9 $\mu$.

On soil, mosses, or decaying wood: Whatcom Co. : Mt. Baker, $2800 \mathrm{ft}$, 1931, 1179. Pierce Co.: Mt. Rainier, Grand Park, 5575 ft., 1931, 654; Berkeley Park, 6000 ft., 1940, 3015. Chelan Co.: Twisp Summit, 6066 ft., 1931, 1318. Ferry Co.: Keller, 2000 ft., 1940, 3117. Yakima Co.: Dewey Lake, 4800 ft., 1940, 2782. Olympic Mountains, Hurricane Ridge, 1935, A. H. Smith 2372 (F). Snohomish Co.: Marysville, 1928, J. M. Grant (WTU).

A common mountain lichen, rather easily identified because of the yellow color reaction with $\mathrm{KOH}$ and the red with $\mathrm{CaOCl}_{2}$.

23. Lecidea holopolia (Tuck.) Zahlbr. Cat. Lich. Univers. $3: 782.1925$.

Biatora holopolia Tuck. Syn. N. Am. Lich. 2:26. 1888.

Thallus brown or olive brown, granulose, granules coarse, tough, round, usually crowded, sometimes scattered. Apothecia circular, 0.3-1.2 mm. in diameter, adnate; disk reddish brown or black, flat becoming convex; proper margin thick becoming lobate; hypothecium hyaline; paraphyses conglutinate, tips forked; asci 8 -spored; spores hyaline, ovoid, guttulate, 9.0-14.0 x 4.0-7.0 $\mu$, description and spore measurements from type specimen.

On old wood: Washington Territory: 1882, Suksdorf 167, Type specimen $(\mathrm{T})$.

24. Lecidea myriocarpella (Merrill) Zahlbr. Cat. Lich. Univers. 3:803. 1925.

Biatora myriocarpella Merrill, Bryol. 16:58. 1913.

Thallus absent or very thin and inconspicuous. Apothecia minute, circular, very numerous, $0.1-0.3 \mathrm{~mm}$. in diameter, adnate to subsessile; disk usually dull black or dark brown, flat to convex; proper margin dull black, thin, soon disappearing; hypothecium hyaline; paraphyses gelatinated, tips dark purplish; asci 8-spored; spores hyaline, ellipsoid, rarely 1-septate, 8.0 x $4.0 \mu$. 
On old wood: Thurston Co.: Gate, 1912, Foster 1987 (F). Herre (1943) reports this species as formerly common on old timbers at Bellingham.

25. Lecidea paddensis (Tuck.) Zahlbr. Cat. Lich. Univers. 2:808. 1925. Biatora paddensis Tuck. Syn. N. Am. Lich. 2:25. 1888.

Thallus yellowish white or greenish gray, granulose, granules flat and tough, scattered. Apothecia circular, 0.4-1.0 mm. in diameter, sessile; disk pale tawny, reddish brown, or black, flat to convex; proper margin concolorous, flexuous, and finally disappearing; hypothecium hyaline; paraphyses conglutinated; asci 8-spored; spores hyaline, globose to ovoid, nonseptate, 6.6-9.9 x 4.9-5.3 $\mu$.

On old wood or bark: Spokane Co.: Mt. Spokane, 5808 ft., 1931, 1490a. Washington Territory, Mt. Paddo, 1883, Suksdorf 164, type specimen $(\mathrm{T})$.

26. Lecidea protabacina Nyl. in Hasse, Bull. S. Calif. Acad. 2:60. 1903.

Thallus yellow, purplish red, or fawn color $(R)$, squamulose, squamules turgid, convex, smooth, shiny, round to angular, edges black, crowded, sometimes scattered, occasionally fissured, on a black hypothallus; underside black. Apothecia circular, 0.4-1.5 mm. in diameter, single or grouped, sessile; disk black, shiny, becoming convex; proper margin blackish, thin, gradually disappearing; hypothecium dark brown; paraphyses closely coherent; asci 8-spored; spores hyaline, oblong-ellipsoid, nonseptate, 10.0$12.0 \times 4.0-5.0 \mu$. Medulla blue with IKI.

On rock: I have seen no specimen of this species from the state. Fink (1935) reports it from Washington.

27. Lecidea tornoensis Nyl. and Saelan, Herb. Mus. Fenn. 1859.

Biatora tornoensis (Nyl. and Saelan) Th. Fr. Nov. Act. Reg. Soc. Sci. Upsal. ser. 3, $3: 196.1860$.

Thallus chocolate, tawny, or olive green, granulose, granules flat, crowded often forming an areolate-rimose crust. Apothecia circular, 0.4-1.0 $\mathrm{mm}$. in diameter, adnate; disk reddish brown or black, convex, becoming tuberculate; proper margin evanescent; hypothecium hyaline or pale yellow; paraphyses coherent, tips dark brown, enlarged; asci 8-spored; spores hyaline, broadly ellipsoid, wall thick, sometimes in two layers, nonseptate, $17.0-23.0 \times 10.0 \mu$.

On bark or old wood: Washington Territory, Suksdorf 102 (T), $50(\mathrm{~S})$.

The rather large thick walled spores distinguish this species.

28. Lecidea varians Ach. Syn. Meth. Lich. 38. 1814.

Biatora varians (Ach.) Eschw. Syst. Lich. 26. 1824.

Thallus grayish white, grayish green, or yellowish green, granulose, 
granules minute, forming a rather thin, smooth crust, bordered and crossed by black hypothalline lines. Apothecia circular, often clustered, minute, 0.1-0.3 mm. in diameter, adnate; disk pale yellow, reddish brown, or black, when black, pruinose, flat to convex; proper margin thin, soon disappearing; hypothecium hyaline; paraphyses conglutinated; asci 8-spored; spores hyaline, ovoid-ellipsoid, nonseptate, 8.0-15.0 x 5.0-6.0 $\mu$.

On bark or old wood: Pacific Co. : Long Beach, 1931, 465. Wahkiakum Co.: Cathlamet, 1940, 2901. Pierce Co.: White River Camp, 3900 ft., 1940, 3093. Yakima Co.: Dewey Lake, 4800 ft., 1940, 2786.

29. Lecidea decipiens (Ehrh.) Ach. Meth. Lich. 80, 1803.

Lichen decipiens Ehrh. Hedw. Descr. and Adumbr. Musc. Frond. 2:7. 1789.

Biatora decipiens (Ehrh.) Fries.

Psora decipiens (Ehrh.) Hoffm.

Thallus flesh color ( $R$ ), coral red ( $R$ ), brick red, or orange shades, squamulose, squamules thin, medium size, smooth, sometimes pruinose, round, irregularly lobed, concave, undulate, closely attached to the substratum, edges white; below white. Apothecia marginal or in center of squamules, 0.4-1.0 mm. in diameter, sessile; disk brown or black, convex; proper margin paler, hardly visible, soon disappearing; hypothecium pale brown; paraphyses conglutinated, tips umber; asci 8-spored; spores hyaline, oblong-ovoid, nonseptate, $12.0-15.0 \times 6.0-7.0 \mu$.

On soil: Washington Territory, Cascade Mountains, 1883, Brandegee $16(\mathrm{~T})$.

A very showy lichen, easily recognized if one is fortunate enough to find it, but not common in the state.

30. Lecidea demissa (Rutström) Ach. Meth. Lich. 81. 1803.

Lichen demissus Rutström, Spicil. P1. Crypt. Suec. 8. 1794.

Biatora atro-rufa (Dicks.) Fries.

Psora demissa (Rutström) Stein.

Thallus grayish white or grayish brown, squamulose, squamules thin, small, round, irregularly lobed, edges crenate, imbricated, squamules sometimes running together into a rough warty crust on a black hypothallus, closely attached to the substratum. Apothecia circular, often clustered and confluent, $0.3-1.0 \mathrm{~mm}$. in diameter, immersed to adnate; disk reddish brown or black, usually flat becoming convex; proper margin darker and soon disappearing; hypothecium hyaline; paraphyses thick, tips brown; asci 8-spored; spores hyaline, ovoid-ellipsoid, nonseptate, 12.0-14.0 x 6.0-8.0 $\mu$.

On soil: Washington Territory, Cascade Mountains, 1883, Brandegee 79 (F).

31. Lecidea globifera Ach. Meth. Lich. 213. 1803.

Biatora globifera Ach.

Psora globifera (Ach.) Mass. 
Thallus olive brown, grayish green, more often chestnut-brown, squamulose, squamules rather large, smooth, somewhat shiny, concave, undulate, irregularly lobed, somewhat imbricated, often ascending at the edges; below gray or white. Apothecia clustered often confluent, 0.8-1.5 $\mathrm{mm}$. in diameter, sessile, elevated; disk black with often a greenish tinge, convex or globose; proper margin lighter in color and soon disappearing; hypothecium pale brown or reddish brown; paraphyses conglutinate; asci 8-spored; spores hyaline, rare, ovoid-ellipsoid, nonseptate, 10.0-14.0 x 6.9-7.0 $\mu$.

On soil: Pierce Co.: Chinook Pass, 5440 ft., 1931, 1557. Yakima Co.: Rattlesnake Canyon, 2000 ft., 1931, 1536. Chelan Co.: Beaver Summit, 2800 ft., 1931, 1335; butte, near Twisp, 1700 ft., 1931, 1459. Whitman Co.: Pullman, $2550 \mathrm{ft} ., 1577$. Washington Territory, Cascade Mountains, 1883, Brandegee 41 ( T).

This is rather a distinctive lichen, because of the large squamules with upturned edges, showing the gray or white under surface.

32. Lecidea lurida (Dill.) Ach. Meth. Lich. 77. 1803.

Lichen luridus Dill. in Wither. A Bot. arrang. Brit. P1. 2:720. 1776.

Biatora lurida (Dill.) Fries.

Psora lurida (Dill.) Lam. and DC.

Thallus some shade of brown, often lurid brown or Mars Brown ( $R$ ), squamulose, squamules smooth or slightly rough, rounded, adnate, crenately lobed, procumbent, imbricated; below pale yellow or light brown. Apothecia circular, scattered or sometimes clustered, $0.1-2.0 \mathrm{~mm}$. in diameter, adnate; disk sometimes concolorous with the squamules, often reddish-brown or black, flat to convex; proper margin brown or black, obtuse, disappearing; hypothecium yellowish brown or brown; paraphyses coherent, tips reddish-brown; asci 8 -spored; spores hyaline, oblong-ovoid, nonseptate, $11.4-16.0 \times 5.0-7.6 \mu$.

On rock or soil: Yakima Co.: Rattlesnake Canyon, 2000 ft., 1931, 1547a. Chelan Co. : butte near Twisp, $1700 \mathrm{ft.}, 1931,1462$.

33. Lecidea ostreata (Hoffm.) Schaer. Lich. Helvet. Spicil. sect. 3. 110. 1828.

Psora ostreata Hoffm. Deutschl. F1. 163. 1796.

Biatora ostreata (Hoffm.) Fries.

Thallus pale tawny, grayish olive (R), or light fawn color (R), squamulose, squamules small, smooth, flat to ascending, imbricated or often scattered, crenately lobed, sometimes much reduced to glebous scales; below white often powdery. Apothecia circular, 0.5-1.2 mm. in diameter, adnate; disk black, slightly white pruinose, flat; proper margin gray, soon becoming black, rather thick, flexuous; hypothecium thick, dark brown; paraphyses colorless, coherent; asci 8-spored; spores hyaline, oblongellipsoid, 8.0-12.0 × 2.5-3.0 $\mu$. 
On burned or old wood. Washington Territory, 1882, Suksdorf $170(\mathrm{~T})$.

34. Lecidea rubiformis Wahl. F1. Lapp. 479. 1812.

Baeomyces rubiformis Wahl. in Ach. Meth. Lich. 324. 1803.

Biatora globifera var. rubiformis (Wahl.) Torss.

Psora rubiformis (Wahl.) Hook.

Thallus fawn colored $(\mathrm{R})$ or brown, squamulose, squamules very thick, smooth, ascending, imbricated, shallow lobed, edges crenate, white; below white. Apothecia circular, clustered often confluent, 0.7-1.0 mm. in diameter, sessile; disk black or purplish black, convex to globose; proper margin thin, disappearing; hypothecium pale brownish; paraphyses coherent, tips reddish brown; asci 8-spored; spores hyaline, oblong-ellipsoid, nonseptate, $11.4-16.0 \times 5.0-7.6 \mu$.

On rock or soil : Chelan Co. : Chelan, $2000 \mathrm{ft} ., 1931,1324$. Stevens Co. : Boyds, 1470 ft., 1931, 1442. Ferry Co.: Republic, Maye's Butte, 1913, Foster 2366 (F). Washington Territory, 1882, Suksdorf 170 (T).

35. Lecidea Russellii Tuck. Proc. Am. Acad. Arts and Sci. 5 :417. 1862.

Biatora Russellii Tuck. Lich. Calif. 23. 1866.

Psora Russellii (Tuck.) Schneid.

Thallus whitish, bright reddish tawny, dull or reddish brown, squamulose, squamules ample, thick, round, somewhat irregular in shape, appressed, margins ascendant, undulate, often furrowed, scattered, or crowded, edges white; below white. Apothecia circular, 0.4-1.2 mm. in diameter, adnate to sessile; disk reddish brown, convex; proper margin lighter in color, obtuse, disappearing; hypothecium yellowish or pale brown; paraphyses coherent; asci 8-spored; spores hyaline, ellipsoid, nonseptate, $9.0-13.0 \times 4.6-6.0 \mu$.

On rock: Washington Territory, Cascade Mountains, 1883, Brandegee $65(\mathrm{~F})$.

32. Mycoblastus Norm. Nyt. Mag. Nat. $7: 24.1852$.

Thallus uniformly crustose, smooth or rather wrinkled and warty, no differentiation into definite layers. Apothecia circular, adnate to sessile, with a proper margin; hypothecium hyaline; paraphyses coherent, branched or unbranched; asci 1-2-spored; spores very large, hyaline, nonseptate.

Alga Protococcus.

A. Medulla blood red beneath the apothecia.

2. M. sanguinarius

A. Medulla not blood red beneath the apothecia. 1. M. alpinus

1. Mycoblastus alpinus (Fr.) Kernst. in Kerner, Schedae ad Fl. AustrHung. 6:121. 1893.

Lecidea sanguinaria var. alpinus Fr. Lich. Europ. Reform. 335. 1831. Heterothecium sanguinarium f. affine (Schaer.) Tuck. Mycoblastus sanguinarius var. alpinus (Fries.) Stein. 
Thallus grayish white or pale greenish gray, smooth and polished to rough and warty, often forming a granular crust, sometimes areolate; medulla not blood red beneath the apothecia. Apothecia scattered, 1.0-2.0 $\mathrm{mm}$. in diameter, circular often irregular, immersed to adnate; disk black and shiny, convex to subglobose; proper margin black with a reddish tinge, soon disappearing; hypothecium hyaline or faintly colored; paraphyses coherent with bluish black tips; spores very large, hyaline, oblongellipsoid, nonseptate, 70.0-95.7 × 20.0-50.0 $\mu$.

On decaying wood and bark: Clallam Co.: Lake Sutherland, $590 \mathrm{ft}$., 1931, 1132. Whatcom Co.: Mt. Baker, Galena, 3800 ft., 1931, 1190. King Co.: Snow Lake Trail, 3000 ft., 1931, 830. Pierce Co.: White River Camp, $3900 \mathrm{ft} ., 1940$, 3093a; Mt. Rainier, Berkeley Park, 6000 ft., 1931, 617, $1940,3024$.

2. Mycoblastus sanguinarius (L.) Norm. Nyt. Mag. Nat. $7: 237.1853$. Lichen sanguinarius Linn. Sp. P1. 1140. 1753.

Heterothecium sanguinarius (L.) Tuck.

Thallus white, grayish white, pale yellow, or dark greenish gray, rough and warty, often forming a granular crust, sometimes broken, areolate; medulla blood red beneath the apothecia. Apothecia scattered, crowded, $1.0-3.0 \mathrm{~mm}$. in diameter, circular, often irregular in shape, immersed or adnate; disk black, often shiny, convex to globose; proper margin black with a reddish tinge, soon disappearing; hypothecium hyaline or faintly colored; paraphyses coherent with bluish black tips; spores very large, hyaline, oblong-ellipsoid, nonseptate, 70.0-92.4 x 20.0-50.0 $\mu$.

On decaying wood and bark: San Juan Co.: Orcas Island, Mt. Constitution, $2500 \mathrm{ft}$., 1928, 2017. Grays Harbor Co.: Lake Quinault, $300 \mathrm{ft}$., 1931, 1207. Kitsap Co.: Hidden Ranch near Bremerton, 213 ft., 1930, 547. King Co.: Snoqualmie Lodge, $3300 \mathrm{ft} ., 1931,1781$. Pierce Co.: trail to Mowich Lake, $3500 \mathrm{ft.,} 1940,2989$, 3064. San Juan Co.: Orcas Island, Turtle Back Mountain, 1906, Fink 469 (W). Clallam Co.: Port Angeles above High Line Logging Cutting, 3000 ft., 1914, Foster 2807, 2812 (W).

\section{Catillaria Th. Fr. Lich. Scand. $1: 563.1871-1874$.}

Thallus crustose throughout or with foliose lobes on the margins, smooth, granulose, warty, or areolate, no definite differentiation into layers. Apothecia circular, immersed, adnate, or sessile; proper margin concolorous with the thallus; hypothecium light colored or black; paraphyses unbranched, free or coherent, sometimes with knob-shaped tips; asci 8-spored; spores hyaline, 1-septate, rarely nonseptate.

Alga Protococcus.

A. Apothecia reddish black or black, one cell of the spore narrower than the other, septum always distinct. 1. C. atropurpurea

A. Apothecia flesh color (R), brown, or black, both cells of the spore equal in size, septum not always distinct. 
B. Disk often more than $1.0 \mathrm{~mm}$. in diameter, tips of paraphyses hyaline, no limiting black hypothallus.................................... 2. C. sphaeroides

B. Disk never more than $1.0 \mathrm{~mm}$. in diameter, tips of paraphyses pale brown, limiting black hypothallus present....................... . C. tricolor

1. Catillaria atropurpurea (Schaer.) Th. Fr. Lich. Scand. 1:565. 1874. Lecidea sphaeroides var, atropurpurea Schaer. Lich. Helvel. Spicil. sect.4-5, 165. 1833.

Biatora atropurpurea (Schaer.) Hepp.

Thallus grayish green or dusky drab ( R), thin, smooth, soon scurfy and granulate, rarely sorediate, sometimes obsolete. Apothecia scattered, circular, very small, $0.4-0.8 \mathrm{~mm}$. in diameter, slightly immersed or adnate; disk reddish brown or black, flat to convex; proper margin tumid, disappearing; hypothecium pale yellow; paraphyses free, pliable; spores hyaline, ellipsoid, 1-septate, one cell narrower than the other, 9.0-16 $\times 3.5-5.9 \mu$.

On bark of trees and old wood: King-Kittitas Co.: Snoqualmie Pass, 3010 ft., 1931, 816. San Juan Co.: Wasp Island, 1906, Fink 180 (W).

2. Catillaria sphaeroides (Mass.) Schuler, Zur Flechtfl. von Fiume, 36. 1902.

Biatorina sphaeroides Mass. Ricerch. Auton. Lich. 135. 1852.

Thallus white, grayish white, or greenish white, effuse or thin, finely granulose often forming a crust. Apothecia scattered, circular, 0.3-1.0 mm. in diameter, adnate or sessile; disk yellowish flesh color, pale reddish brown, or orange cinnamon (R), flat to convex; proper margin not visible; hypothecium hyaline; paraphyses free with knob-shaped tips; spores hyaline, ellipsoid, 1-septate, 10.0-13.2 × 2.9-3.3 $\mu$.

On moss or wood: San Juan Co.: Turn Island, 1928, 2018. Ferry Co.: Republic, 1912, Foster (F).

3. Catillaria tricolor (With.) Th. Fr. Lich. Scand. 1:574. 1871-1874.

Lichen tricolor With. Bot. arr. Brit. P1. 3rd ed., 4 :23. 1796.

Biatora mixta Fries.

Thallus grayish white, smooth to slightly granulate, chinky to warty, usually with a limiting and decussating black hypothallus. Apothecia scattered, circular, 0.2-0.6 mm. in diameter, adnate; disk flesh color (R), reddish brown turning black, flat to slightly convex; proper margin paler, persistent for a long time, finally disappearing; hypothecium hyaline; paraphyses slender, free, thread-like, with knob-shaped slightly brownish tips; spores hyaline, ellipsoid or fusiform, 1-septate, 9.0-16.0 × 2.0-6.6 $\mu$.

On bark and dead wood: San Juan Co.: Pt. Caution, 1928, 1936; Brown Island, 1928, 2020; Friday Harbor, 1906, Fink 13, 60, 141 (W).

34. Bacidia (DeNot.) Zahlbr. Nat. Pfl. Teil 1, Abt. 1, 135. 1907. DeNot., in Giorn. Bot. Ital. 2 :189. 1846. 
Thallus crustose, granulose, or powdery, sometimes subsquamulose or areolate, smooth or rough, no definite differentiation into layers. Apothecia scattered, circular, rarely immersed, usually adnate, sometimes sessile; proper margin concolorous with the thallus, usually disappearing; hypothecium rarely hyaline, usually pale yellow or brown; paraphyses unbranched, free or coherent, tips often knob-like; asci usually 8-spored, rarely 16 -spored; spores hyaline, 2 -many-septate, with a gelatinous halo, sometimes one end of the spores prolonged into a tail.

Alga Protococcus.

A. On moss or soil, hypothecium brown. 1. B. sabuletorum

A. On trees, hypothecium hyaline or yellowish brown.

B. Hypothecium hyaline, disk pale flesh color $(\mathrm{R})$ or salmon color.

B. Hypothecium hyaline or yellowish brown.
C. Disk always black.
2. B. akompsa
C. Disk never black, reddish yellow or reddish brown.
4. B. luteola

1. Bacidia sabuletorum (Schreb.) Lettau, Hedwigia 52:132. 1912.

Lichen sabuletorum Schreb. Spicil. Fl. Lips. 134. 1771.

Biatora hypnophila (Turn.) Lönnr.

Bilimbia sabuletorum (Schreb.) Arn.

Thallus gray or greenish gray, of thin, crowded, sometimes confluent granules forming a crust, sometimes disappearing. Apothecia circular, often clustered, 0.2-0.8 mm. in diameter, adnate or sessile; disk pale yellow, tawny, or black, flat to convex; proper margin black soon disappearing; hypothecium dusky brown; paraphyses with brownish knob-like tips; asci 8-spored; spores hyaline, ellipsoid or fusiform, 3-7-septate, 16.5-29.7 x 3.7-6.6 $\mu$.

On moss or soil: Yakima Co.: Little Naches River, 2557 ft., 1931, 1002a. San Juan Co.: Roche Harbor, 1906, Fink 246 (W). Rainier National Park, J. M. Grant 433 (F).

2. Bacidia akompsa (Tuck.) Fink, Cont. U.S. Nat. Herb. 14 :90. 1910.

Biatora akompsa Tuck. Syn. N. Am. Lich. 2:47. 1888.

Thallus pale yellow or ash colored, of minute granules forming a crust, smooth or rough and warty. Apothecia circular, 0.2-0.6 $\mathrm{mm}$. in diameter, adnate or sessile; disk black, rough, flat to subglobose; proper margin thin, uneven; hypothecium hyaline or yellowish brown; paraphyses coherent, hair-like; asci 8-spored; spores hyaline, acicular, 3-5-septate, 18.0-22.0 x 1.5-2.5 $\mu$, measurements made from type specimen, Biatoria akompsa $2872(\mathrm{~T})$.

On trees: Islands of Puget Sound, 1906, E. T. Harper. Longmire Springs, 1906, E. T. Harper, Fink (1919).

3. Bacidia albescens (Krmphbr.) Zwackh. Flora $45: 495.1862$. 
Scoliciosporum molle f. albescens Krumphbr. Denkschr. Kgl. Bayer.

Bot. Gesellsch. 4 :pars, 2 :207. 1861.

Thallus pale yellowish white or greenish gray, not always very distinct, thin, granulose or powdery. Apothecia circular, 0.3-0.7 mm. in diameter, adnate or sessile; disk waxy brown, pale flesh color $(R)$, or salmon color $(\mathrm{R})$, flat to convex; proper margin finally disappearing; hypothecium hyaline; paraphyses coherent, with knob-like tips; asci 8-spored; spores hyaline, acicular, 3-many-septate, septa often obscure, 20.0-44.0 x 1.2-2.0 $\mu$.

On trees: Whatcom Co.: 1914, A. C. Herre (F).

4. Bacidia luteola (Schrad.) Mudd, Manual Brit. Lich. 183. 1861.

Lichen luteolus Schrad. Spicil. Fl. Ger. 85. 1794.

Biatora rubella (Ehrh.) Rabh.

Thallus granulose or scurfy sometimes disappearing, white, pale yellow, or greenish gray. Apothecia circular, $0.5-1.2 \mathrm{~mm}$. in diameter, adnate or sessile; disk ochraceous-salmon ( $\mathrm{R})$, reddish yellow or reddish brown, sometimes obscurely white-pruinose, flat to convex, proper margin thin, finally disappearing; hypothecium pale or yellowish brown; paraphyses loosely coherent, slender; asci 8-spored; spores hyaline, acicular, 5-manyseptate, $45-60 \times 2-4 \mu$.

On trees: Washington Territory, Suksdorf 297 (S).

\section{Toninia Mass. Richerch. Anton. Lich. 107. 1852.}

Thallus crustose, squamulose, or subfoliose, inflated and subpedicellate, marginally lobed, thin gelatinized upper cortex, rather distinct algal and medullary layers. Apothecia circular, adnate to sessile; proper margin variously colored; hypothecium light or dark; paraphyses free or coherent, unbranched, tips often knob-like; asci 8-spored; spores hyaline, 1-7- or rarely more septate.

Alga Protococcus.

A. Spores 3-septate; thallus often a grayish white crust......1. T. aromatica A. Spores 3-many-septate; thallus never a grayish white crust.

B. Disk concave, papillate; squamules with a stem-like projection.

2. $T$. caulescens

B. Disk flat to convex, not papillate; squamules without a stem-like projection.

C. Squamules usually tawny brown, coarsely reticulated and pitted.

3. T. ruginosa

C. Squamules usually citrine-drab ( $R)$, neither reticulated nor pitted.

4. T. squarrosa

1. Toninia aromatica (Turn.) Fram. Lich. 4. 1855.

Lichen aromaticus Turn. in Sm. and Sowerb. Eng. Bot. 25: tab. 1777. 1807.

Lecidea aromatica Turn. 
Thallus of grayish white, crumb-like or powdery granules, or of brown to greenish black, small, thick, crowded squamules. Apothecium circular, often irregular, usually clustered, 0.5-1.5 mm. in diameter, adnate; disk black, flat to convex, proper margin black, thin, soon disappearing; hypothecium pale brown; paraphyses violet, free, globose tipped; spores hyaline, fusiform with obtuse ends, 3-septate, $18-29 \times 3-5 \mu$.

On rocks, also on mosses growing over rocks: Klickitat Co.: Bickleton, $3200 \mathrm{ft}$., 1931, 1641.

2. Toninia caulescens Anzi Cat. Lich. Sondr. 67. 1860.

Lecidea caulescens (Anzi) Tuck. Gen. Lich. 182. 1872.

Lecidea squalida var. caulescens (Anzi) Nyl.

Thallus of tawny-brown or greenish brown, turgid, convolute, crowded and imbricated squamules, the squamules often extending downward in pale, brownish stems. Apothecia circular or lobulate, 0.5-2.0 mm. in diameter, sessile; disk black, papillate, concave; proper margin black, conspicuous, turgid, undulate; hypothecium bright reddish brown; paraphyses free, with brownish knob-like tips; spores hyaline, acicular or narrowly fusiform, 3-many-septate, $25-40 \times 3-5 \mu$.

On soil and rocks: Klickitat Co.: Bickleton, $3200 \mathrm{ft} ., 1931,1641 a$. Washington Territory, Cascade Mountains, 1883, Brandegee 7 (S). Klickitat Co.: Goldendale, 1909, Foster 1627 (F).

3. Toninia ruginosa (Tuck.) Herre, Proc. Wash. Acad. Sci. 12:103. 1910. Lecidea ruginosa Tuck. Lich. Calif. 25. 1866.

Thallus of green or tawny brown, crowded, round, turgid, glebous, rough, wavy, folded, finally reticulated, rarely perforate squamules. Apothecia circular, undulate, somewhat irregular, 0.5-3.0 $\mathrm{mm}$. in diameter; disk dull black, flat to slightly convex; proper margin black, thick, flexuous, finally disappearing; hypothecium brownish; paraphyses free or loosely coherent, with thickened brownish tips; spores hyaline, acicular, 3-many-septate, $23.1-35.0 \times 3.5-4.0 \mu$.

On rocks, also moss and soil: Chelan Co.: Rainbow Trail, 3500 ft., 1931, 1261. Washington Territory, Suksdorf 226 (S).

4. Toninia squarrosa (Ach.) Th. Fr. Lich. Scand. $1: 331.1874$.

Lecidea atrorufa var. squarrosa Ach. Kgl. Vet.-Akad. Nya Handl. 267. 1808.

Lecidea squalida Ach.

Thallus of citrine-drab (R), brown, or black, small, thick, appressed, imbricated, somewhat lobed, rugose-plicate squamules. Apothecia subglobose, sometimes irregular, numerous, often confluent, $0.8-2.0 \mathrm{~mm}$. in diameter, subsessile; disk black, flat to convex; proper margin thin, soon disappearing; hypothecium very pale yellow; paraphyses coherent, with 
violet knob-like tips; spores hyaline, acicular or fusiform, 3-many-septate, 26.4-62.0 x 3.3-5.0 $\mu$.

On soil, moss over rock, or rock: Chelan Co.: Beaver Summit, $2800 \mathrm{ft}$, 1934, 2474a. Yakima Co.: Naches Ranger Station, 2400 ft., 1940, 3322. Ferry Co. : Mt. Gibraltar, 3100 ft., 1940, 3167. Ferry Co.: Republic, 1912, Foster 2329a (F). Washington Territory, Cascade Mountains, Brandegee ( T).

36. Lopadium Körb. Syst. Lich. Ger. 210. 1855.

Thallus uniformly crustose, no differentiation into definite layers. Apothecia circular, sessile or substipitate; proper margin light or dark in color, carboniferous, soft, or cartilaginous; hypothecium hyaline or dark brown; paraphyses free or coherent, branched or unbranched; asci 1-8-spored, with very thick walls; spores hyaline, straight or curved, muriform, many celled, thin walled without a halo.

Alga Protococcus.

1. Lopadium pezizoideum (Ach.) Körb. Syst. Lich. Ger. 210. 1855.

Lecidea pezizoidea Ach. Lichenogr. Univers. 182. 1810.

Thallus consisting of greenish white, pale yellow, or brown, small, flat squamules or granules, sometimes warty or coralloid. Apothecia circular, 0.4-1.2 $\mathrm{mm}$. in diameter, sessile; disk black, flat to concave; proper margin brownish black, thick, entire, finely wrinkled, rarely disappearing; hypothecium dark brown; paraphyses coherent, black at the tips; asci 1-spored; spores hyaline, oblong ellipsoid, large, muriform, many celled, 40.0-72.6 x 20.0-40.0 $\mu$.

On trees and encrusting mosses on rocks: Clallam Co.: Port Angeles, Little River Logging Co., 2000+ ft., 1914, Foster 2811 (W) ; Olympic Hot Springs, 3500 ft., 1935, A. H. Smith 1967 (F). Washington Territory, Suksdorf 214 (T).

37. Rhizocarpon Lam. in Lam. and DC. Fl. Franc. 3rd ed., 2:365. 1805.

Thallus crustose, smooth or warty, often areolate, rarely squamulose, no distinct differentiation into definite layers, a black hypothallus usually present. Apothecia circular or irregularly angulate, immersed or sessile; proper margin black or brown; hypothecium dark; paraphyses branched, coherent or loose and distinct; asci 1-8-spored; spores hyaline, more often brown, 1-many-septate transversely, or muriform, with a distinct muscilaginous halo.

Alga Protococcus.

A. Spores 1-septate; asci 8-spored.

1. R. badioatrum

A. Spores 3-septate or muriform; asci 1, 2, or 8-spored.

$\mathrm{B}$. Thallus with $\mathrm{KOH}$ turns yellow then red.

3. R. chlorophaeum

$\mathrm{B}$. Thallus does not react with $\mathrm{KOH}$ as above; mature spores all muriform. 
C. Asci 8-spored.

D. Areoles citrine yellow or greenish yellow......5. R. geographicum

D. Areoles not citrine yellow or greenish yellow.

E. Thallus smooth to areolate, gray or brown ......... $R$. $R$. petraeum

E. Thallus areolate, gray or brown, but always with a purple tinge.

6. $R$. grande

C. Asci 1 or 2 -spored; spores usually more than $40 \mu$ long.

F. Thallus squamulose, chestnut-brown or black....... 2. R. Bolanderi

$F$. Thallus areolate, gray or brown.......................... 4. R. disporum

1. Rhizocarpon badioatrum (Flk.) Th. Fl. Lich. Scand. 1:613. 1871-1874.

Lecidea badioatra Flk. in Sprgl. Neue Entdeck. 2:95. 1821.

Buellia badioatra (Flk.) Körb. Syst. Lich. Ger. 223. 1855.

Thallus gray, deep slaty brown ( $R$ ), brown, or reddish brown, determinate, thick, areolate or cracked areolate, flat to convex, closely crowded forming a crust or scattered over the black hypothallus. Apothecia circular, 0.5-1.0 $\mathrm{mm}$. in diameter, slightly immersed or adnate; disk black, flat, proper margin slightly raised, concolorous with the disk; hypothecium dark brown; paraphyses coherent, tips bluish black; asci 8-spored; spores brown, hyaline when young, oblong or oblong-ellipsoid, 1-septate, sometimes slightly constricted in the middle, $26.0-38.0 \times 12.3-18.0 \mu$.

On rocks: San Juan Co.: Orcas Island, Mt. Constitution, $2500 \mathrm{ft}$, 1928, 2019. Yakima Co.: Bumping Lake, 3400 ft., 1931, 922. Ferry Co.: Laurier, 1644 ft., 1931, 1445. Olympic Mountains, Boulder Peak, 5500 ft., 1935, A. H. Smith 2895. (F).

2. Rhizocarpon Bolanderi (Tuck.) Herre Proc. Wash. Acad. Sci. 12:106. 1910.

Buellia Bolanderi Tuck. Gen. Lich. 189. 1872.

Thallus chestnut brown or black, indeterminate, of small to medium sized squamules, flat to slightly concave, shiny, slightly wavy, margins ascending, under side black, which often shows as an elevated black border, because of the ascending margins. Apothecia circular, 0.2-1.0 mm. in diameter, adnate or sessile; disk dull black, naked, flat to convex; proper margin concolorous with the disk, thin, wavy, finally disappearing; hypothecium dark brown; paraphyses coherent, tips dark brown with a violet tinge; asci 2 -spored; spores hyaline, grayish or dark brown, oblong-ellipsoid, muriform, 34.6-50.0 x 17.1-23.1 $\mu$.

On rocks: Yakima Co. : gulch near Naches, 1350 ft., 1931, 908. Klickitat Co.: Bickleton, $3000 \mathrm{ft} ., 1931,1668$. Okanogan Co.: gulch near Tonasket, $1200 \mathrm{ft} ., 1931,1386$. Washington Territory, 1882, Suksdorf 201 (S).

3. Rhizocarpon chlorophaem (Hepp.) Müll. Arg. in Engl. Bot. Jahrb. $15: 519.1893$.

Lecidea chlorophaea Hepp in Leight. Lich.-Fl. Gr. Brit. 328. 1871. 
Thallus creamy white or tawny, thin or moderately thick, warty or cracked areolate, areoles unequal in size, continguous or scattered, reaction with $\mathrm{KOH}$ yellow then red. Apothecia numerous, 0.2-0.5 mm. in diameter, innate or subsessile; disk black, flat to convex, slightly pruinose; proper margin grayish, finally disappearing; hypothecium pale brown; paraphyses loose, with brown knob-like tips; asci 8-spored; spores grayish, oblongellipsoid, 3-septate or muriform, 16.5-19.8 x 6.6-9.9 $\mu$.

On rocks: San Juan Co.: San Juan Island, Pt. Caution, 1928, 1947a.

A very rare species, easily determined by the yellow and then red color change with $\mathrm{KOH}$.

4. Rhizocarpon disporum (Naeg.) Müll. Arg. Revue Mycolog. 1:170. 1879.

Lecidea dispora Naeg. in Hepp, Flecht. Europ. No. 28. 1853.

Thallus gray or brownish, indeterminate, areoles scattered or contiguous, rather round, flat to convex, on an indistinct black hypothallus. Apothecia circular, $0.5-0.8 \mathrm{~mm}$. in diameter, sessile; disk black, flat to slightly convex; proper margin slightly elevated, entire, persistent; hypothecium dark brown; paraphyses coherent, tips violaceous black, asci 1 or 2spored; spores hyaline at first, becoming brownish gray or dark brown, oblong-ellipsoid, large, muriform, 26.4-60.0 x 13.2-30.0 $\mu$.

On rocks: Yakima Co.: gulch near Naches, 1350 ft., 1931, 1174. Chelan Co.: Twisp Trail, $4000 \mathrm{ft} ., 1931,1298$. Okanogan Co.: gulch near Tonasket, $1200 \mathrm{ft} ., 1931,1383$.

5. Rhizocarpon geographicum (L.) DC. in Lam. and DC. Fl. Franc. 3rd ed., $2: 365.1805$.

Lichen geographicus Linn. Sp. P1. 1140. 1753.

Buellia geographica (L.) Tuck.

Thallus citron yellow $(R)$ or clear dull green yellow ( $R$ ), determinate, thin to moderately thick, warty, or areolate, areoles flat to slightly convex, smooth, usually contiguous, rarely somewhat scattered on a black hypothallus. Apothecia angular, usually crowded, 0.4-1.0 mm. in diameter, partly immersed between the areoles; disk black, flat; proper margin concolorous with the disk, thin, finally disappearing; hypothecium dark brown; paraphyses loosely coherent, with brownish black tips; asci 8-spored; spores dark brown, oblong-ellipsoid, muriform, 17.0-40.0 x 11.0-20.0 $\mu$.

On rocks: Pierce Co. : Mt. Rainier, Berkeley Park, 6000 ft., 1940, 3029. Yakima Co.: Dewey Lake, 4800 ft., 1940, 2790; American River Camp, $2800 \mathrm{ft} ., 1931$, 957. Jefferson Co. : Mt. Olympus, on the summit, $8200 \mathrm{ft}$., 1906, Frye 72 (W). Olympic Mountains, Boulder Peak, $5500 \mathrm{ft} ., A$. H. Smith, 2859 (F). Whitman Co.: Pullman, R. K. Beattie 53 (F).

A very conspicuous alpine rock lichen, easily recognized by the yellow thallus with the black apothecia immersed in it. 
6. Rhizocarpon grande (Flk.) Arn. Flora $54: 149.1871$.

Lecidea petraea var. fuscoatra f. grandis Flk. in Fw. Flora $11: 690.1828$.

Buellia petraea var. grandis (Flk.) Tuck.

Thallus deep violet-gray $(\mathrm{R})$ or brown with a purplish tinge, determinate, thin to moderately thick, areoles small, convex, scattered on a prominent black hypothallus. Apothecia 0.4-1.0 mm. in diameter; disk black, convex to subglobose; proper margin black, thin, soon disappearing; hypothecium dark brown; paraphyses coherent, tips brownish or bluish black; asci 8-spored; spores hyaline to brown, oblong-ellipsoid, muriform, 26.4-40.0 x 13.2-16.5 $\mu$.

On rocks: Yakima Co.: gulch near Naches, 1350 ft., 1940, 3191. Chelan Co.: butte near Chelan, 2000 ft., 1931, 1343a. Ferry Co.: Sanpoil, $2000 \mathrm{ft} ., 1940$, 3128; Laurier, $1644 \mathrm{ft} ., 1931,1445 \mathrm{a}$. Stevens Co.: Boyds, $1470 \mathrm{ft} ., 1931,1452$. Spokane Co.: west of Spokane, $1950 \mathrm{ft} ., 1940$, 2750 .

This species is common east of the Cascades. It resembles $R$. petraeum, but can be separated from that species by the characteristic violet or purple tinge of the thallus.

7. Rhizocarpon petraeum (Wulf.) Mass. Ricerch. Auton. Lich. 102. 1852. Lichen petraeus Wulf. Schrift. Gesellsch. nat. Freund. Berlin, 3:89. 1787.

Buellia petraea var. vulgaris Tuck. Gen. Lich. 190. 1872.

Thallus grayish white, gray, or brown, determinate, thin, smooth or warty, sometimes areolate; areoles continuous or scattered on a black hypothallus which is often inconspicuous. Apothecia circular or angular, crowded, 0.3-1.0 mm. in diameter, immersed or adnate; disk black or brownish black, flat or convex; proper margin black, thick, elevated, entire or irregular, sometimes white pruinose; hypothecium dark brown; paraphyses somewhat distinct; asci 8-spored; spores hyaline or brown, oblongellipsoid, muriform, $18.0-39.0 \times 8.0-18.0 \mu$.

On rocks and more rarely on old wood: Okanogan Co.: gulch near Tonasket, $1200 \mathrm{ft} ., 1931,1387$. 


\section{CLADONIACEAE}

Thallus usually twofold, primary thallus crustose, granulose, squanulose, or foliose, often soon disappearing, more or less differentiation into layers, attached to the substratum by hyphal rhizoids or occasionally by true rhizoids; secondary thallus consisting of erect podetia, short or elongated, simple or branched, corticate or non-corticate and hollow or solid. Apothecia borne on the tips of the podetia or laterally, globose, cylindrical, or irregular in shape; proper margin well developed, thalline margin usually lacking; asci 6-8-spored; spores hyaline, non-many-septate, or muriform.

Alga Protococcus, rarely a blue-green.

A. Podetia short, usually simple, rarely branched; apothecia terminal.

B. Apothecia black; hypothecium dark colored. 39. Pilophoron

B. Apothecia light colored; hypothecium hyaline.

38. Baeomyces

A. Podetia longer, usually much branched; apothecia terminal or lateral.

C. Cephalodia rare; podetia usually hollow, spores non-septate.

40. Cladonia

C. Cephalodia common; podetia solid, spores 3-more-septate.

41. Stereocaulon

38. Baeomyces Ehrh. Beitr. zur Nat. 4 :149. 1789.

Thallus crustose, granulose, or squamulose, containing a thin upper cortex of gelatinous hyphae, an algal and a medullary layer, but no lower cortex, attached to the substrate by hyphal rhizoids. Podetia very short, arising from the medullary layer of the thallus (endogenous), alga present or absent. Apothecia globose or irregular in shape, light colored, single or conglomerate, terminal on the podetia or rarely sessile on the thallus; proper margin present or apothecia becoming hemispherical and immarginate; hypothecium hyaline; paraphyses unbranched, distant; asci 8 -spored; spores hyaline, nonseptate or 1-3-septate.

Alga Protococcus or occasionally a blue-green.

1. Baeomyces rufus (Huds.) Rebent. Prodro. Fl. Neomarch. 315. 1804. Lichen rufus Huds. Fl. Angl. 443. 1762.

Baeomyces byssoides (L.) Gärt.

Thallus greenish gray, pale yellowish gray, or grayish white, a rather 
thin effuse crust, granulose becoming scurfy squamulose or crenatelobulate. Podetia flattened and furrowed, rarely branched near the tips, granulose or corticate, $1-3 \mathrm{~mm}$. in length, sometimes very short or entirely wanting, concolorous with the thallus. Apothecia subglobose or flattened, becoming flexuous, $1.0-1.5 \mathrm{~mm}$. in diameter; disk pale flesh color (R), strongly convex, proper margin soon disappearing; asci 8-spored; spores hyaline, ellipsoid, nonseptate, $6.0-15.0 \times 3.0-4.0 \mu$.

On soil or rock: Wahkiakum Co.: Cathlamet, 1907, Foster 486 (W), this specimen determined as Baeomyces roseus by G. K. Merrill. Herre (1917) reports this species as common in the wooded lowlands of Whatcom Co., Clallam Co.: Sequim, 1918, J. M. Grant (F).

39. Pilophoron Th. Fr. Stereo. and Pilophor. Comment. 40. 1857.

Primary thallus crustose, without differentiation into definite layers, attached to the substratum by hyphal rhizoids. Podetia arising from the primary thallus, decorticate and usually granulose, covered with thalloid warts, the center consisting of loosely packed, longitudinally extending hyphae, surrounded by a region of densely packed hyphae. Apothecia terminal, globose, subglobose, or subcylindrical, black; proper margin soon disappearing; hypothecium brown; paraphyses unbranched, septate, dark blue and thickened at the tips; asci 8-spored; spores hyaline, nonseptate. Alga Protococcus.

A. Apothecia large, cylindrical or almost cylindrical...................2. P. Hallii A. Apothecia smaller, globose or subglobose.

1. P. cereolus

1. Pilophoron cereolus (Ach.) Th. Fr. Lich. Scand. 1:55. 1871-74.

Lichen cereolus Ach. Lichenogr. Suec. Prodro. 89. 1798.

Primary thallus greenish gray, gray, or light brownish olive, forming a warty or powdery crust. Podetia arising from the primary thallus, erect, cylindrical, simple or branched, surface warty or powdery. Apothecia terminal, black, 0.4-2.0 mm. in diameter, subglobose; asci 8-spored; spores hyaline, ellipsoid, nonseptate, $16.0-23.0 \times 5.0-8.0 \mu$.

On rock or on soil over rock: Clallam Co. : Lake Crescent, 483 ft., 1931, 1035. Pacific Co.: Ft. Canby, 1930, 467. San Juan Co.: Orcas Island, Mt. Constitution, $2500 \mathrm{ft} ., 1931$, 2079. Whatcom Co. : Baker Lake, 1941, E. B. Mains, 6219 (F).

An odd, unusual looking lichen, easily recognized, that always makes me think of a black headed pin, found only west of the Cascades.

2. Pilophoron Hallii (Tuck.) Wainio Bot. Mag. Tokyo 35 :59. 1921.

Pilophoron cereolus b. Hallii Tuck. Syn. N. Am. Lich. $1: 235.1882$.

Primary thallus greenish with green stripes, forming a warty or powdery crust. Podetia greenish gray or light brownish olive (R), arising from the primary thallus, erect, subcylindrical, solid, short and stout, seldom branched, minutely granulose or powdery. Apothecia terminal, vertically 
elongated, $2.0-6.0 \times 0.5-1.0 \mathrm{~mm}$. in diameter, almost cylindrical, pestleshaped; asci 8-spored; spores hyaline, fusiform or ellipsoid, sharp pointed, 19.0-24.0 x 4.0-7.0 $\mu$.

On rock: Pierce Co.: trail to Mowich Lake, $3500 \mathrm{ft} ., 1940,3061$. Olympic Mountains, near Humes Glacier on Mt. Olympus, $5000 \mathrm{ft} . ; 1907$, Frye 26 (W). Clallam Co.: Olympic Hot Springs, 1914, Foster 2886 (W). Mason Co. : Mt. Elinor, 1912, Foster 2096 (W).

This is also an unmistakable lichen with its cylindrical or pestle-shaped apothecia.

40. Cladonia (Hill) Web. Hill, Hist. P1. 91. 1751 in part. Web. in Wigg. Prim. P1. Hols. 90. 1780.

Primary thallus crustose, foliose, or squamulose, persistent or dying, upper cortex present, lower cortex absent, algal and medullary layers well defined, often sorediate, attached to the substratum by hyphal rhizoids. Podetia radial, erect, arising from the primary thallus, differentiated into an outer cortex containing algae, and an inner medullary layer, usually hollow in the center, subcylindrical or widening towards the top to form cups, simple or branched, decorticate, corticate, or sorediate, cortex smooth, warty, or composed of continuous or scattered areoles, often dying at the base. Apothecia of various shapes and sizes, terminal on the podetia or on the cups or branches, sometimes short stalked; proper margin thin, soon disappearing; hypothecium hyaline or colored; paraphyses unbranched, agglutinated, rarely forked; asci 6-8-spored; spores hyaline, usually nonseptate, some species 1-3-septate. A large and difficult genus.

Alga Protococcus or Cystococcus.

Subgenus I. Cladina (Nyl.) Wainio-Primary thallus crustose, soon disappearing, seldom seen. Podetia much and intricately branched, often with the whorls of three or more branches, surrounding gaping axils; no true cortex, surface arachnoid, without squamules or soredia; cupless. Apothecia rarely seen; disk brown.

A. Podetia in dense, irregularly branched masses.

B. Podetia usually grayish, sometimes with greenish or brownish tips, ultimate branches blunt, usually in groups of $3-6, \mathrm{KOH}+, \mathrm{P}+$.

3. Cl. rangiferina

B. Podetia usually straw-yellow ( $R$ ), sometimes with a pale greenish tinge or whitish, tips of ultimate branches pointed, usually in groups of $3-8$, with frequent branches between the whorls.

C. Podetia often with a greenish tinge, $\mathrm{KOH}-, \mathrm{P}+\ldots . . .4 \mathrm{Cl}$. sylvatica

C. Podetia usually whitish, $\mathrm{KOH}-, \mathrm{P}-\ldots \ldots \ldots \ldots \ldots \ldots \ldots \ldots \ldots . .2 . \mathrm{Cl}$. mitis

A. Podetia in rather regular, smooth, compact masses, almost white or whitish gray, with a straw-yellow $(\mathrm{R})$ cast, $\mathrm{KOH}-, \mathrm{P}-$.

1. Cl. alpestris

Subgenus II. Pycnothelia Ach.-Primary thallus granular-crustose, persistent. Podetia short not more than 1 inch long, stout, simple or with 
short branches, terminating in blunt points. Apothecia small; disk reddish-brown. At present the only known species in this subgenus is $\mathrm{Cl}$. papillaria. So far as the writer knows it has never been found in the state.

Subgenus III. Cenomyce (Ach.) Th. Fr.-Primary thallus squamulose or foliose, persistent, or sometimes disappearing. Podetia branched or unbranched, never very intricately branched. Apothecia usually present, sometimes lacking; disk scarlet or various shades of brown.

\section{Section 1. Cocciferae Del.}

Disk of apothecia scarlet, or rarely flesh-color (R), orange or pale yellowish brown.

A. Podetia cylindrical, cupless.

B. Podetia short and stout, usually decorticate, farinose-sorediate, $\mathrm{KOH}+, \mathrm{P}+$. 23. Cl. macilenta

B. Podetia rather slender, usually decorticate, farinose-sorediate, $\mathrm{KOH}-, \mathrm{P}-$.

5. Cl. bacillaris

A. Podetia cup-bearing.

C. Podetia short, not often more than $2 \mathrm{~cm}$. long.

D. Podetia powdery sorediate, $\mathrm{KOH}+, \mathrm{P}+$

15. Cl. digitata

D. Podetia not sorediate, $\mathrm{KOH}-, \mathrm{P}-$.

11. Cl. coccifera

C. Podetia long and narrow, more than $2 \mathrm{~cm}$. long.

E. Podetia corticate, densely squamulose, sparingly sorediate, $\mathrm{KOH}-\mathrm{P}$ -

6. $\mathrm{Cl}$. bellidiflora

E. Podetia partly decorticate, sparingly squamulose, abundantly sorediate.

F. Podetia slender, granular sorediate, $\mathrm{KOH}+, \mathrm{P}+$.

26. Cl. polydactyla

F. Podetia stouter, yellowish green, farinose-sorediate. $\mathrm{KOH}-$, $\mathrm{P}-$. 14. Cl. deformis

Section 2. Ochrophaeae Wainio

Disk of apothecia pale or dark shades of brown.

A. Primary thallus always present, squamules large and foliose, sometimes bearing black fibrils on the margins. $\mathrm{KOH}-, \mathrm{P}+$. 17. $\mathrm{Cl}$. foliacea

A. Primary thallus not always present, squamules small to medium sized.

B. Podetia without cups.

C. Cortex smooth and continuous or nearly so.

D. Podetia yellowish green or dark olive $(R)$, rather short, very

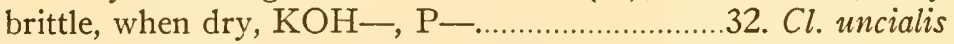

D. Podetia greenish gray, reddish brown, or brownish olive $(R)$, elongated, not noticeably brittle when dry.

E. Sparingly squamulose, $\mathrm{KOH}-, \mathrm{P}+\ldots \ldots \ldots \ldots \ldots \ldots . . .18 . \mathrm{Cl}$. furcata

E. Squamulose only towards the base, $\mathrm{KOH}+$, bright yellow turning pinkish red, $\mathrm{P}+$. 30. Cl. subsquamosa 
E. Abundantly squamulose.

F. $\mathrm{KOH}+$, bright yellow turning pinkish red, $\mathrm{P}+$.

31. Cl. subsquamosa f. luxurians

F. $\mathrm{KOH}-, \mathrm{P}+$. 19. Cl. furcata var. pinnata

C. Cortex rough, uneven and fissured, $\mathrm{KOH}+, \mathrm{P}+\ldots \ldots \ldots$. 7. $\mathrm{Cl}$. cariosa B. Podetia with cups.

G. Cups perforate.

$\mathrm{H}$. Podetia brown towards the apex, cups closed by membranes that are often radially lacerate, $\mathrm{KOH}-, \mathrm{P}+\ldots \ldots 24 . \mathrm{Cl}$. multiformis

$\mathrm{H}$. Podetia not brown near the apex, cups closed by membranes that are punctured with regular holes.

I. Podetia densely covered with squamules.

J. Podetia $\mathrm{KOH}-, \mathrm{P}+$ faintly.

29. Cl. squamosa

J. Podetia $\mathrm{KOH}+$ bright yellow turning pinkish red, $\mathrm{P}+$.

31. Cl. subsquamosa f. luxurians

I. Podetia bearing rather coarse scattered squamules.

$\mathrm{K}$. Podetia long, usually unbranched, $\mathrm{KOH}-\mathrm{P}+$.

9. $\mathrm{Cl}$. cenotea

K. Podetia shorter, usually branched, $\mathrm{KOH}-, \mathrm{P}+$.

13. Cl. crispata

I. Podetia bearing squamules only near the base.

30. Cl. subsquamosa

G. Cups not perforate.

L. Podetia flesh color (R) or fawn color (R), $\mathrm{KOH}-, \mathrm{P}+$.

8. Cl. carneola

L. Podetia some shade of brown, gray, or green.

M. Podetia corticate, smooth and continuous, or of dispersed areoles, not sorediate.

N. Cups shallow, saucer form.

O. Proliferations from margins of cups.

P. Cups rather broad, podetia rather short, $\mathrm{KOH}+$, or-; $\mathrm{P}+$. 20. $\mathrm{Cl}$. gracilis

P. Cups narrow, sometimes lacking, or often lacking.

Q. Podetia often cupless, very long.

22. $\mathrm{Cl}$. gracilis var. elongata

Q. Podetia usually cupless, not very long.

21. $\mathrm{Cl}$. gracilis var. chordalis

O. Proliferations chiefly from the center of cups.

R. Proliferations always from the center of cups, forming a series of $4-5$ cups, $\mathrm{KOH}-\mathrm{P}+$.

34. $\mathrm{Cl}$. verticellata var. evoluta

R. Proliferations from center and margins of cups, also from sides of podetia, $\mathrm{KOH}-, \mathrm{P}+$.

33. $\mathrm{Cl}$. verticellata

N. Cups deep, goblet form.

S. Primary squamules rather thin and somewhat incised, ascending, $\mathrm{KOH}-, \mathrm{P}+$.

27. $\mathrm{Cl}$. pyxidata 
S. Primary squamules rather thick, nearly entire, packed into a crust. 28. $\mathrm{Cl}$. pyxidata var. pocillum M. Podetia wholly or partly decorticate, sorediate.

T. Cups definite and well developed.

U. Podetia sorediate-granular, usually stout, $\mathrm{KOH}-, \mathrm{P}+$. 10. $\mathrm{Cl}$. chlorophaea

$\mathrm{U}$. Podetia sorediate-farinose, slender, $\mathrm{KOH}-, \mathrm{P}+$.

16. Cl. fimbriata

T. Cups absent, or irregular and poorly developed.

V. Podetia sorediate throughout, $\mathrm{KOH}-, \mathrm{P}+$.

12. Cl. coniocraea

$\mathrm{V}$. Podetia on the upper part only, granular-sorediate, $\mathrm{KOH}-\mathrm{P}+$. 25. Cl. nemoxyna

1. Cladonia alpestris (L.) Rabh. Clad. Eur. Exs. No. 11. 1860.

Lichen rangiferinus var. alpestris Linn. Sp. P1. 1153. 1753.

Cladonia rangiferina $\mathrm{c}$. alpestris $\mathrm{L}$.

Podetia nearly white or whitish gray, with a straw yellow cast, surface arachnoid or almost tomentose, in older parts the branches look translucent, branching dichotomous or whorled, surrounding gaping axils. Podetia of rather even length, producing a smooth, compact topped mass, the most striking characteristic of the species. $\mathrm{KOH}-, \mathrm{P}-$, no color changes with either.

On soil: not collected by the author. Grays Harbor Co.: Westport, 1908, Foster 675 (W) ; Pacific Beach, Foster 1412 (F).

In some regions this species is called, by children, castles or biscuits, which seems to me to be a good description.

2. Cladonia mitis Sandst. in Abh. Nat. Ver. Bremen. 25 :105. 1922.

Podetia straw yellow ( $R$ ), or more often creamy white, or white, surface never distinctly arachnoid; no further attempt will be made to distinguish this species morphologically from $\mathrm{Cl}$. sylvatica, which it certainly resembles very closely. But since I have used chemical reactions to help distinguish species of Cladonias, it seems correct to recognize $\mathrm{Cl}$. mitis as a species. $\mathrm{KOH}-, \mathrm{P}-$, no color change with either.

On soil: San Juan Co.: San Juan Island, Friday Harbor, 1928, 1957. Ferry Co.: Laurier, 1644 ft., 1931, 1422. San Juan Co.: San Juan Island, 1906, Fink 155 (W), determined by Fink as Cl. sylvatica.

3. Cladonia rangiferina (L.) Web. in Wigg. Prin. Fl. Hols. 90. 1780.

Lichen rangiferimus Linn. Sp. P1. 1153. 1753.

Podetia grayish white, ashy gray, greenish, or with a brownish tinge, surface decidedly arachnoid, especially on the lower branches, forming a loose and gray cottony background for the green areoles, branching dichotomous or whorled, surrounding gaping axils, the tips of the ultimate 
branches usually in groups of 3-6, all tending to curve in one direction, tips more or less brown in color. $\mathrm{KOH}+$, yellow, $\mathrm{P}+$, reddish orange.

On soil: San Juan Co.: Brown Island, 1940, 3208. King Co.: Chase Bog, 1940, 3337. Snow Lake Trail, 3500 ft., 1931, 827. Pierce Co.: Silver Springs, 2670 ft., 1931, 1118. Clallam Co.: Olympic Hot Springs, 1936, A. H. Smith 2118 (F).

The grayish color of this plant and the nodding tips of the branches help to distinguish this species; also yellow color with $\mathrm{KOH}$.

4. Cladonia sylvatica (L.) Hoffm. Deutschl. Fl. 114. 1795.

Lichen rangiferinus var. sylvaticus Linn. Sp. P1. 1153. 1753.

Cladonia rangiferinus b. sylvatica $\mathrm{L}$.

Podetia straw yellow $(\mathrm{R})$, creamy white, sometimes with a grayish or pale greenish tinge, surface distinctly arachnoid, but less strikingly so than in $\mathrm{Cl}$. rangiferina, sometimes becoming weathered and verruculose, branching dichotomous or whorled, surrounding gaping axils, with frequent subsecund branches between the whorls on the main axes, thus making the branching very dense, the tips of the ultimate branches usually in groups of 3-8, inclined to be straight, sometimes brown, more often concolorous with the rest of the podetia. $\mathrm{KOH}-, \mathrm{P}+$, reddish orange.

On soil: San Juan Co.: San Juan Island, Friday Harbor, 1940, 3212, Brown Island, 1940, 3209. Mason Co.: Mt. Elinor, 5500 ft., 1912, Foster 2113 (W). Grays Harbor Co.: Copalis, 1911, Foster 1428 (W).

The yellow color, almost white when dry, helps to distinguish this species from $\mathrm{Cl}$. rangiferina, also the negative reaction with $\mathrm{KOH}$; but no doubt the two are often confused.

5. Cladonia bacillaris (Ach.) Nyl. Not. Sällsk. F. et Fl. Fennica Förhandl. $8: 179$ (footnote). 1866.

Baeomyces bacillaris Ach. (in parte) Meth. Lich. 329. 1803.

Primary thallus rarely dying, grayish green or grayish olive (R), consisting of squamules, lacinate lobed or margin crenate, flat or ascending, scattered or crowded, under surface white. Podetia whitish, grayish green, or grayish olive $(R)$, cylindrical, slender, mostly decorticate and farinose sorediate, sometimes corticate near the base and below the apothecia, bearing a few squamules; cupless or rarely with imperfect cups, simple or sometimes branched near the apex, often sterile with obtuse or subulate tips. Apothecia scarlet, solitary or clustered, 1.0-3.0 mm. across; disk irregularly convex. $\mathrm{KOH}-, \mathrm{P}-$, no color changes with either.

On rotten stumps or logs, rarely on soil: King Co. : Edgewood, $300 \mathrm{ft}$., 1940, 3079. San Juan Co.: San Juan Island, Friday Harbor, 1906, Fink 50 (W). Herre (1917) reports it from the lowlands of Whatcom County.

6. Cladonia bellidiflora (Ach.) Schaer. Lich. Helv. Spic. 21. 1823.

Lichen bellidiflorus Ach. Lich. Suec. Prodro. 194. 1798. 
Primary thallus rarely persistent, when present, pale brown or greenish gray, consisting of lobed or crenately margined squamules, sorediate, under surface white or slightly brownish. Podetia concolorous with the primary thallus, sometimes brown or black at the base, rather elongated, cylindrical, erect, somewhat flexuous, simple or branched, cortex continuous or broken and dispersed in the form of areoles, bearing crowded or small scattered squamules; cups small, not always present, margins entire or dentate. Apothecia clustered, $1.0-4.0 \mathrm{~mm}$. across, often on the tips of the branch or on the margin of the cup; disk scarlet, flat or convex. $\mathrm{KOH}-, \mathrm{P}-$, no color changes with either.

On soil, rock, or decaying wood: San Juan Co.: Shaw Island, 1928, 2077; Brown Island, 1940, 3210; San Juan Island, Trout Lake, 1940, 3237. Pierce Co.: Mt. Rainier, Summerland Trail, 3500 ft., 1931, 692; Mt. Rainier, trail to Mowich Lake, 3500 ft., 1940, 3069; Mt. Rainier, Van Trump Park, 5000 ft., 1942, 3619. San Juan Co.: Orcas Island, Mt. Constitution, $2500 \mathrm{ft}$., 1906, Fink 328 (W). Jefferson Co.: Olympic Mts. near Humes Glacier, 5000 ft., 1907, Frye 80 (W) ; Boulder Peak, 5500 ft., 1935, A. H. Smith 2911 (F).

7. Cladonia cariosa (Ach.) Spreng. Syst. Veg. $4: 272.1827$.

Lichen cariosa Ach. Lich. Suec. 198. 1798.

Primary thallus ustually persistent, grayish olive ( $R$ ) or greenish gray, consisting of squamules, ascending or erect, with crenately lobed margin, somewhat involute, under surface white, sparingly sorediate. Podetia grayish white or greenish gray, appearing hoary or half dead, clustered, short, stout, erect or ascending, club shaped, laterally torn, grooved and fissured, branching abundantly, cortex continuous or subcontinuous and dispersed in the form of areoles; cupless. Apothecia on the tips of the branches, clustered, often conglomerate, sometimes $4.0 \mathrm{~mm}$. across; disk brown or reddish brown, flat to convex, $\mathrm{KOH}+$, bright yellow, $\mathrm{P}+$, reddish orange.

On soil or rock: Kittitas Co.: Easton, 2168 ft., 1931, 849. Yakima Co.: American River Camp, 2800 ft., 1931, 946. Chelan Co.: Stehekin Valley, 1140 ft., 1931, 1291. Ferry Co.: Gibraltar Mt., 3783 ft., 1940, 3151. Whitman Co.: Tekoa Mts., 2900 ft., 1931, 1596. Clallam Co.: Mt. Angeles, 1911, Foster 1793 (W), determined by Merrill as Cladonia cariosa f. cribosa (Wainio) Spreng. Thurston Co.: Gate, 1912, Foster 1923 (W). Determined by G. K. Merrill as Cladonia cariosa f. cribosa.

A small lichen usually found growing on the earth, easily recognized by the grooved and fissured podetia; rather widely distributed in the state.

8. Cladonia carneola Fries Lich. Eur. Ref. 233. 1831.

Cenomyce carneola Fries Sched. Crit. fasc. 4 :23. 1824.

Primary thallus persistent or sometimes dying, greenish gray or straw yellow ( $R$ ), consisting of crenately lobate squamules, flat or ascending, margins involute, underside white or straw yellow ( $R$ ), somewhat soredi- 
ate. Podetia concolorous with the primary thallus, clustered, more rarely solitary, medium length, cylindrical or top shaped, erect, hollow, branched, entirely or partly corticate toward the base, toward the top cortex, dispersed in the form of areoles and sorediate, squamules very small or entirely absent; cups small to medium sized, abruptly dilated, margin entire or dentate, proliferate. Apothecia on short stipe from the margin of the cups, clustered or solitary, clusters often $6.0 \mathrm{~mm}$. across; disk flesh color (R), fawn color $(\mathrm{R})$, rarely brick-red, flat to convex. $\mathrm{KOH}-, \mathrm{P}+$, slightly reddish orange.

On soil, a thin layer of soil over rocks, or decaying wood: San Juan Co.: San Juan Island, trail to Heaven, 1940, 3247. Pierce Co.: Mt. Rainier, Summerland, 6500 ft., 1931, 688. Clallam Co. : Port Angeles, Bird's, 1914, Foster 2600 (W) ; Olympic Hot Springs, 1914, Foster 2875 (W). Jefferson Co.: Queets River Valley, near Humes Glacier, 5000 ft., 1907, T. C. Frye $32(\mathrm{~F})$.

Easily identified by the flesh color or fawn color of the disk, which is unusual for a Cladonia; also by the stipitate apothecia growing from the margins of the cups.

9. Cladonia cenotea (Ach.) Schaer. Lich. Helv. Spic. 1:35. 1823.

Baeomyces cenoteus Ach. Meth. Lich. 345. 1803.

Primary thallus usually persistent, greenish gray or light brownish olive $(\mathrm{R})$, consisting of small or medium sized squamules, flat or ascending, irregularly incised or entire, underside white and somewhat sorediate. Podetia concolorous with the primary thallus, usually clustered, long, erect, subcylindrical, irregular, swollen, rarely branched, upper part decorticate and sorediate, often corticate and squamulose toward the base; cups medium sized, often funnel shaped, perforate, margins proliferate. Apothecia on the proliferations from the margins of the cups, solitary or clustered, small and inconspicuous, often not more than $.5 \mathrm{~mm}$. across, sometimes entirely absent; disk flesh color $(R)$, at length dark brown, flat to convex. $\mathrm{KOH}-, \mathrm{P}+$, faintly reddish orange.

On soil and rotten wood: Not collected by the author. Spokane Co.: Mt. Carlton, 1906, T. A. Bonser (F).

10. Cladonia chlorophaea (Flk.) Spreng. in Linn. Syst. Veg. 4: 273. 1827.

Cenomyce chlorophaea Flk. in Somrft. Suppl. Fl. Lapp. 130. 1826.

Cladonia pyxidata var. chlorophaea Flk.

Primary thallus usually persistent, ashy, greenish, brownish, or olivaceous, consisting of medium sized squamules, flat or ascending, crenate or digitately lobed, concave or convex, underside white or yellowish, sorediate. Podetia concolorous with the primary thallus, solitary or clustered, erect, rather short, cylindrical, usually simple, corticate surface being somewhat areolate or verruculose; cups deep goblet form gradually widening from the base, dentate or proliferating from the margin, bearing either cups or apothecia on the margins, abundant granular-soredia are borne on both the 
inside and outside of the cups, these decorticate areas appear whitish. Apothecia often absent, when present sessile on the margins of the cups, or shortly stipitate, rather small and inconspicuous, sometimes conglomerate, then as much as $4.0 \mathrm{~mm}$. across; disk brown, flat to convex. $\mathrm{KOH}-, \mathrm{P}+$, brick red.

On soil, decaying wood, or on thin layer of soil over rock: Clallam Co.: West Trail Elwha River, 1940, 2867b. Whatcom Co.: Mt. Baker, Galena, 3800 ft., 1931, 1184. Thurston Co. : Grand Mound, 1940, 2948. Pierce Co. : White River Camp, 3900 ft., 1940, 3100; Mt. Rainier, Van Trump Park, 5000 ft., 1942, 3621 ; Berkeley Park, 6000 ft., 1940, 3022. Ferry Co.: Sanpoil, 2360 ft., 3135; Gibraltar Mountain, 3783 ft., 1940, 3144. Grays Harbor Co.: Copalis, 1910, Foster 1417 (W). San Juan Co.: Cypress Island, 1928, 1962.

The deep goblet form cups, the brick red color with paraphenylenediamine, and the brown apothecia when present, the abundant soredia, inside and outside the cups, make this an easy species to recognize. One of the common and widely distributed lichens of this state.

11. Cladonia coccifera (L.) Willd. F1. Berol. Prodro. 361. 1787.

Lichen coccifera Linn. Sp. P1. 1151. 1753.

Cladonia cornucopioides (L.) Fries.

Primary thallus usually persistent, grayish green or yellowish green, consisting of lacinate-lobed or crenate margined squamules, flat, slightly involute, rarely sorediate on the margins, underside white or yellowish white, rarely sorediate. Podetia concolorous with the primary thallus, often solitary, sometimes clustered, short, erect, hollow, cylindrical, usually simple, corticate throughout, not sorediate, areoles or verrucoles may be somewhat separated in the upper part, rarely squamulose; cups deep, gradually dilating from a narrow base, proliferate, bearing either cups or apothecia on the proliferations. Apothecia on the proliferations of the cups, solitary or clustered, rather large, often $6.0 \mathrm{~mm}$. across; disk scarlet, convex. $\mathrm{KOH}-, \mathrm{P}-$, no color change with either.

On soil and rarely on decaying wood: San Juan Co.: San Juan Island, 1930, 510. Kitsap Co.: Hidden Ranch near Bremerton, 213 ft., 1931, 1720. Mason Co.: Mt. Elinor, 1500 ft., 1912, Foster 2115 (W). Thurston Co.: Gate, 1912, Foster 2004 (W). Spokane Co.: Spokane, 1908, T. A. Bonser 55 (W).

This Cladonia is easily determined by the scarlet apothecia, growing on the margins of the rather broad and deep cups.

12. Cladonia coniocraea (F1k.) Spreng. in Linn. Syst. Veg. 16th ed., $4: 272$. 1827.

Cenomyce coniocraea Flk. Deutschl. Lich. 138. 1821.

Cladonia fimbriata var. coniocraea (Flk.) Wainio.

Primary thallus usually persistent, green, gray, olive-buff $(R)$, or light brown, consisting of digitately lobed, medium sized squamules, flat or 
ascending, involute, underside white. Podetia whitish or grayish, solitary or clustered, rather short, erect, cylindrical, straight and sometimes branching toward the apex, often decorticate through its entire length, more or less densely covered with pale yellow or pale green soredia, toward the base there may be a continuous cortex with a few squamules; cups often abortive, nothing more than truncate apices depressed in the center, when present very narrow. Apothecia rare, if present sessile or stipitate on the margins of the cups, or on the apices of the podetia, small and inconspicuous; disk brown, flat to convex. $\mathrm{KOH}-\mathrm{P}+$, brick red.

On soil and decaying wood: King Co.: Edgewood, 300 ft., 1931, 1809. King-Kittitas Co. : Snoqualmie Pass, 3010 ft., 1931, 818. Chelan Co. : Rainbow Trail, $3800 \mathrm{ft} .$, 1931, 1277. San Juan Co.: San Juan Island, 1906, Fink 148 (W). Determined by Fink as Cladonia fimbriata var. coniocraea. Olympic Mountains, Hurricane Ridge, 1935, A. H. Smith 1135 (F).

Rather an inconspicuous Cladonia, with no very definite distinguishing characteristics.

13. Cladonia crispata (Ach.) Flot. Merkw. Flech. Hirschb. 4. 1839.

Baeomyces turbinatus var. crispatus Ach. Meth. Lich. 341. 1803.

Cladonia furcata var. crispata (Ach.) Flk.

Primary thallus persistent or dying, gray, greenish gray, or greenish brown, consisting of lacinate or crenate, medium sized squamules, ascending, rather densely grouped forming a crust, underside white. Podetia whitish, gray, greenish gray, dull olive green, or brownish green, solitary or clustered, short or medium in length, subcylindrical, erect, branched, axils dilated, cortex continuous, or dispersed in the form of areoles, squamules absent or rather abundant, and conspicuous, because of the prominent clear white under surface, especially noticeable in dried specimens; cups small, dilated, perforate, when cups are not produced, there are dilated and gaping axils. Apothecia on the tips of proliferations growing from the margins of the cups or stipitate on the margins of the cups, usually solitary sometimes clustered, small, seldom more than $0.8 \mathrm{~mm}$. across; disk brown, flat to convex. $\mathrm{KOH}-, \mathrm{P}+$, deep reddish orange.

On soil and decaying wood: Kitsap Co.: Hidden Ranch, near Bremerton, 213 ft., 1931, 1734, 1754. San Juan Co. : San Juan Island, 1906, Fink 170 (W). King Co. : Kent, 1927, G. N. Jones 304 (W).

\section{Cladonia deformis (L.) Hoffm. Deutschl. Fl. 2:120. 1795. \\ Lichen deformis Linn. Sp. Pl. 1152. 1753.}

Primary thallus often dying, if present rather sparse, pale reddish brown or yellowish green, consisting of crenate or lobed, medium sized squamules, underside white somewhat brownish, often sorediate. Podetia straw yellow (R), or green brownish toward the base, usually clustered, sometimes scattered, elongated, cylindrical, often swollen, simple, rarely branched, lower part corticate, sometimes squamulose, upper part sorediate or sorediate throughout, cortex often badly cracked; cups gradually or abruptly dilated, 
margins dentate to torn or irregularly proliferate, mouths sorediate. Apothecia on the margins of the cups or on the tips of the proliferations, solitary or clustered, small to medium sized, 0.5-3.0 mm. across; disk scarlet, irregularly convex. $\mathrm{KOH}-, \mathrm{P}-$, no color changes with either.

On soil and decaying logs: Pierce Co.: Mt. Rainier, Berkeley Park, 6000 ft., 1940, 3011. Yakima Co. : Little Naches, 2557 ft., 1931, 1002.

The stout podetia and the abruptly dilated cups, with dentate to torn or irregularly proliferate margins and sorediate mouths, make this a distinctive Cladonia.

15. Cladonia digitata (L.) Schaer. Lich. Helv. Spic. 22. 1823.

Lichen digitatus Linn. Sp. P1. 1152. 1753.

Cladonia digitata (L.) Hoffm.

Primary thallus usually persistent, grayish green, grayish olive $(R)$ or Isabella color $(R)$, consisting of crenate or lacinate-lobed, small or large and prominent squamules, flat to often strongly ascending, involute, often markedly sorediate on the margins, underside white, sorediate. Podetia whitish, yellowish-white, or greenish-gray, usually clustered, rather short, erect, or ascending, cylindrical, simple, rarely branched, sometimes corticate throughout, more often corticate only near the base, upper part sorediate, sometimes squamulose near the base; cups medium sized, dilated abruptly, margin dentate, proliferate, or almost entire. Apothecia solitary or clustered on the tips of the proliferations or rarely on the margins of the cups, small to medium sized, 0.5-4.0 mm. across; disk scarlet, irregularly convex. $\mathrm{KOH}+, \mathrm{P}+$, deep reddish orange.

On soil and decaying logs: Kittitas Co.: Easton, $2168 \mathrm{ft} ., 1931,850$. San Juan Co.: Orcas Island, Turtle Back Mountain, 1906, Fink 460 (W). Puget Sound, 1865, Dr. Torrey (T).

The short podetia, with apothecia borne on the proliferations, help to characterize this species.

16. Cladonia fimbriata (L.) Fries Lich. Eur. Ref. 222. 1831.

Lichen fimbriatus Linn. Sp. P1. 1152. 1753.

Cladonia fimbriata var. simplex f. minor Wainio.

Primary thallus usually persistent, gray, olive-buff ( R), or light brown, consisting of medium sized, digitately lobate squamules, flat to ascending, margins involute, underside white, sorediate, especially near the margin. Podetia concolorous with the primary thallus, solitary or clustered, erect to irregularly curved, cylindrical, usually decorticate and sorediate throughout, rarely corticate near the base and below the cups, cortex areolate, warty without squamules or rarely squamulose near the base; cups well developed, gradually dilated or occasionally flaring abruptly at the mouth, often sorediate inside, margins dentate. Apothecia sessile or with a short stalk on the margin of the cup, small and inconspicuous; disk brown or reddish brown. $\mathrm{KOH}-, \mathrm{P}+$, bright orange red. 
On soil or decaying wood: Kitsap Co.: Hidden Ranch near Bremerton, 213 ft., 1930, 1960. King Co.: Richmond Highlands, 300 ft., 1931, 577. Clallam Co.: Dean's, Ennis Creek, foot of Mt. Angeles, 1911, Foster 1801 (W). Mt. Rainier, Glacier Basin, 1925, J. M. Grant 416 (F).

The sorediate-farinose podetia, bearing normal regular shaped cups, help to distinguish this Cladonia.

\section{Cladonia foliacea (Huds.) Willd. Fl. Berol. Prodro. 362. 1787. \\ Lichen foliaceus Huds. F1. Ang1. 457. 1762. \\ Cladonia foliacea var. alcicornis (Lightf.) Schaer.}

Primary thallus persistent and very conspicuous, greenish gray or olivebuff $(\mathrm{R})$, consisting of large leaflike, concave squamules, ascending, closely clustered, with deeply laciniate margins, black fibrils sometimes appear on the margins, underside white or cream colored, this underside shows up very plainly in dried specimens. Podetia concolorous with the primary thallus, short, cylindrical, hollow, scattered, erect, cortex, subcontinuous or areolate, rarely squamulose toward the base; cupbearing or cupless, cups small, irregular, margin almost entire to dentate, proliferate. Apothecia on margin of cups, rather rare; disk brown or brick red. $\mathrm{KOH}-$, or faintly yellowish brown, $\mathrm{P}+$, orange or brick red.

On soil or decaying logs : Pierce Co.: Mt. Rainier, Moraine Park Trail, $3300 \mathrm{ft} ., 1940$, 2992; trail to Seattle Park, $4000 \mathrm{ft} ., 1940$, 3056. Yakima Co.: Dewey Lake, 4800 ft., 1940, 2771. Okanogan Co., Twisp Trail, 5000 ft., 1931, 1307. Mason Co.: Mt. Elinor, 1912, Foster 2118 (W).

The persistent, large and conspicuous, leaf-like squamules of the primary thallus make this an easy species to recognize.

18. Cladonia furcata (Huds.) Schrad. Spic. Fl. Germ. 107. 1794.

Lichen furcatus Huds. Fl. Angl. 458. 1762.

Primary thallus soon disappearing, pale greenish gray or brown, consisting of small to medium sized, elongated, narrow, lobed squamules, margins crenate or sinuate, but only seen in young plants. Podetia olive-buff $(\mathrm{R})$, greenish gray, or reddish brown, usually clustered, erect, slender, cylindrical, or subcylindrical, rather intricately dichotomously or radiately branched, often with corymbose clustered tips that are obtuse, acute, or attenuate, axils irregularly gaping and sometimes dilated, cortex continuous or areolate, sparingly squamulose or entirely without squamules; cups entirely absent. Apothecia small to medium sized, borne on the tips of the branches of the podetia; disk pale brown, brown, or reddish brown. $\mathrm{KOH}-, \mathrm{P}+$, brick red.

On soil, on thin layer of soil over rock, or rarely on decaying wood: Pacific Co.: Long Beach, 1940, 2766. San Juan Co.: San Juan Island, Pt. Caution, 1928, 2068; Friday Harbor, 1940, 3213. Clallam Co. : Lake Sutherland, 590 ft., 1931, 1026. Thurston Co.: Gate, 1940, 2964. King Co.: Edgewood, 300 ft., 1931, 1820. Clallam Co.: Port Angeles, Bird's, 1914, 


\section{PLATE SECTION}

Plate I

A. Graphis scripta x 5 .

B. Graphis scripta var. pulverulenta $\times 2$.

C. Lobaria oregana $\times 11 / 2$.

D. Lobaria pulmonaria upper surface $\mathrm{x} 3 / 4$.

\section{Plate II}

A. Lobaria pulmonaria lower surface $\mathrm{x} 3 / 4$.

B. Sticta anthraspis upper surface $\times 1 / 2$.

C. Sticta anthraspis lower surface $\mathrm{x} 1 / 2$.

D. Sticta anthraspis lower surface natural size.

\section{Plate III}

A. Solorina crocea $\times 2$.

B. Peltigera canina $x 3 / 1$.

C. Peltigera canina $\times 2$.

D. Lecidea cyanea natural size.

Plate IV

A. Lecidea cyanea $\times 3 \frac{1}{2}$.

B. Lecidea globifera $\times 2$.

C. Stereocaulon tomentosum natural size.

D. Parmella physodes $\times 1 / 2$.

\section{Plate V}

A. Parmelia physodes $f$. rugosa f. rugosa $\times 1 / 3$.

B. Parmelia vittata $\times 3 / 4$.

C. Parmelia tubulosa $\times 2$.

D. Parmelia perlata upper surface $\times 1 / 3$.

\section{Plate VI}

A. Parmelia perlata lower surface $\times 1 / 3$.

B. Parmelia sulcata $\times 1 / 3$.

C. Cetraria glauca $\times 1 / 3$.

D. Cetaria glauca upper surface $\mathrm{x} \% 3$.

\section{Plate VII}

A. Cetraria glauca lower surface $\mathrm{x} 3 / 4$.

B. Cetraria glauca upper surface $\mathrm{x} 3 / 4$.

C. Evernia prunastri $\times 1 / 2$.

D. Letharia vulpina $\times 1 / 3$.

\section{Plate VIII}

A. Alectoria jubata $\times 1 / 2$.

B. Alectoria sarmentosa $\times 1 / 5$.

C. Alectoria sarmentosa $\times 11 / 2$.

D. Cornicularia californica $\times 2$.

\section{Plate IX}

A. Cornicularia californica $\times 4$.

B. Cornicularia divergens $\times 21 / 2$. not reported from Washington.

C. Cornicularia normoerica $\times 3$.

D. Cornicularia tenuissima $\times 21 / 2$.

\section{Plate X}

A. Ramalina reticulatat $\times 1 / 4$.

B. Ramalina reticulata $\times \frac{2}{3}$.

C. Ramalina reticulata $\times 11 / 2$.

D. Ramalina farinacea $\times 1 \frac{112}{2}$.

Plate XI

A. Usnea hirta $\times 2 / 3$.

B. Usnea longissima $\times 1 / 2$.

C. Usnea ceratina $\times 11 / 3$.

D. Caloplaca murorum $\times 4$. 


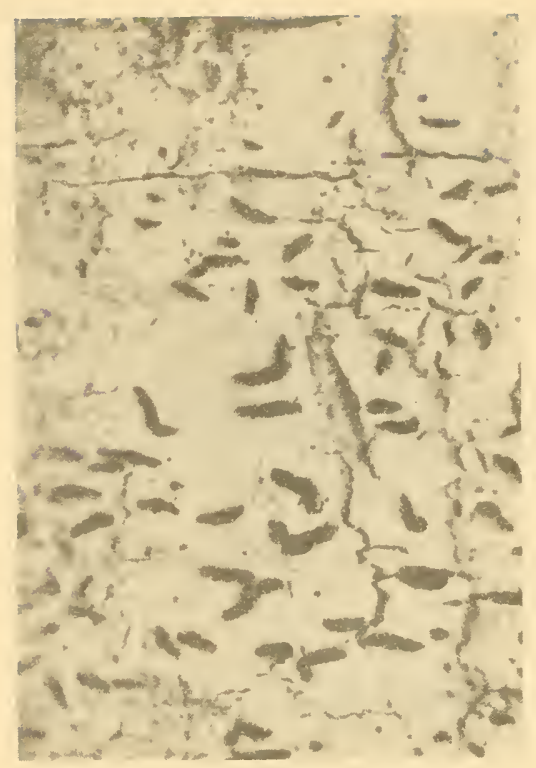

A

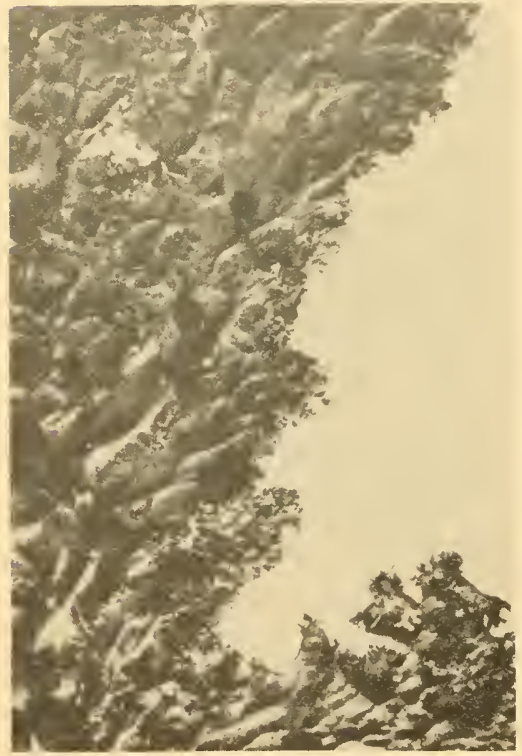

C

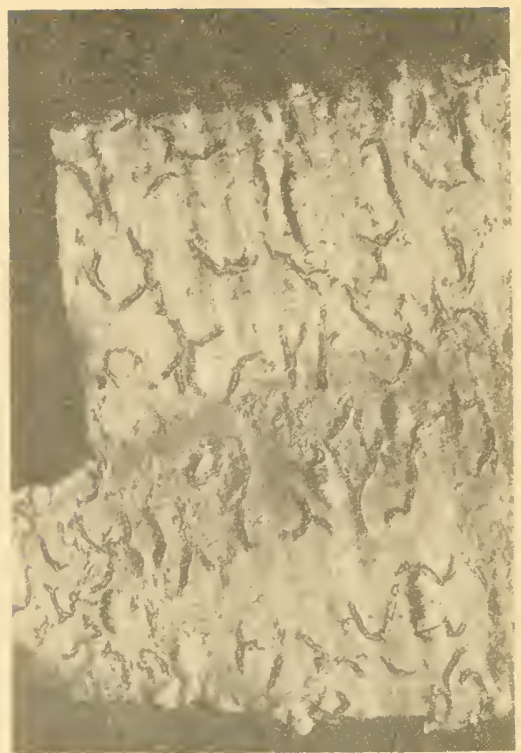

B

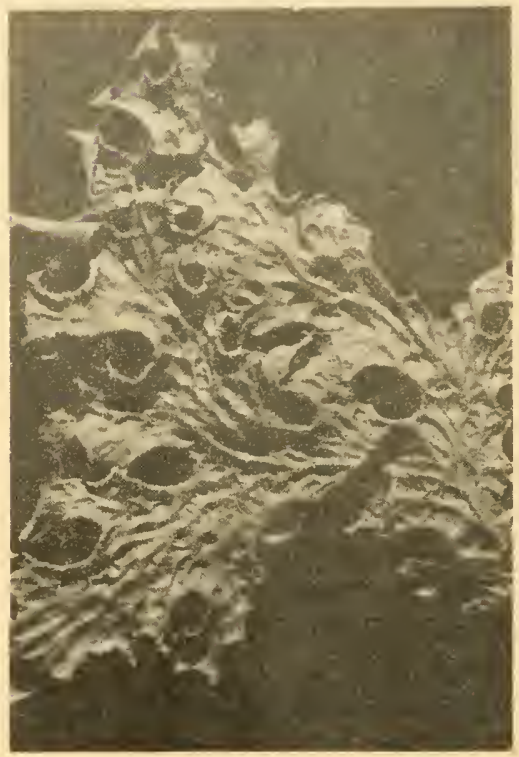

D 


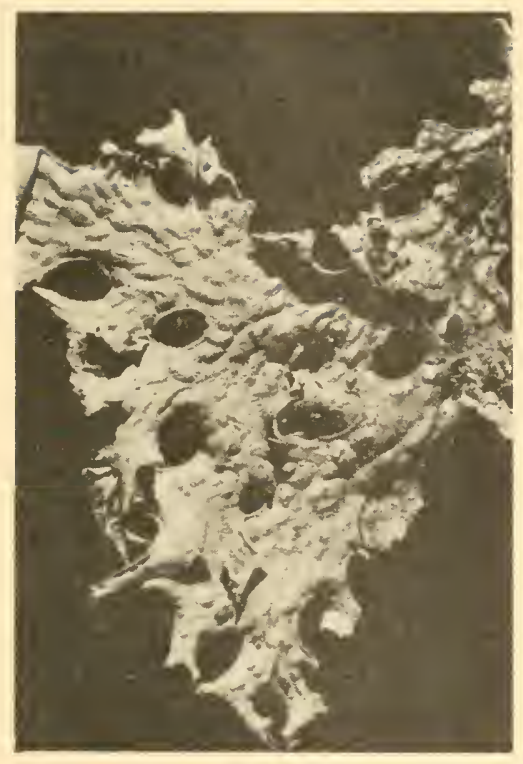

A

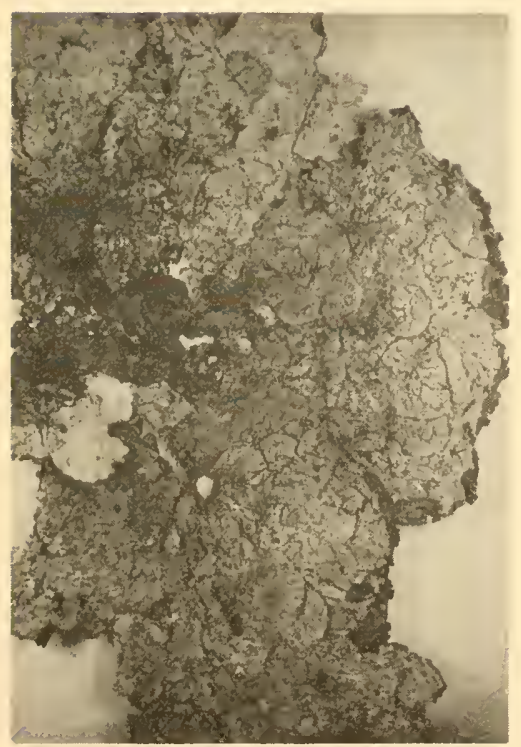

C

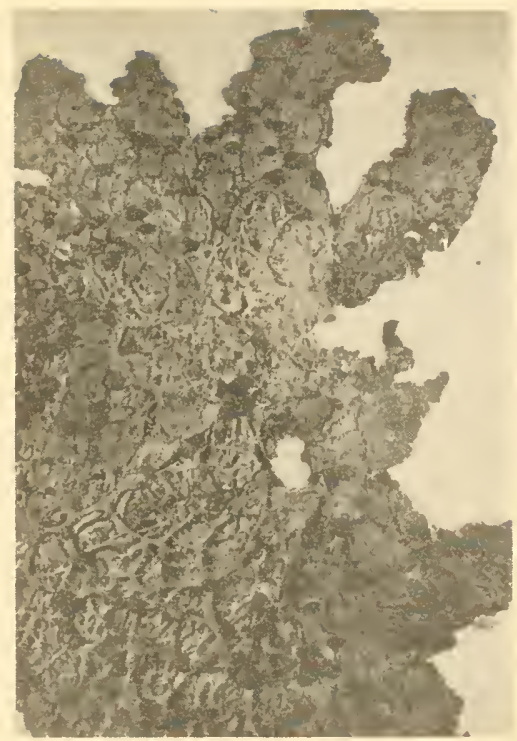

B

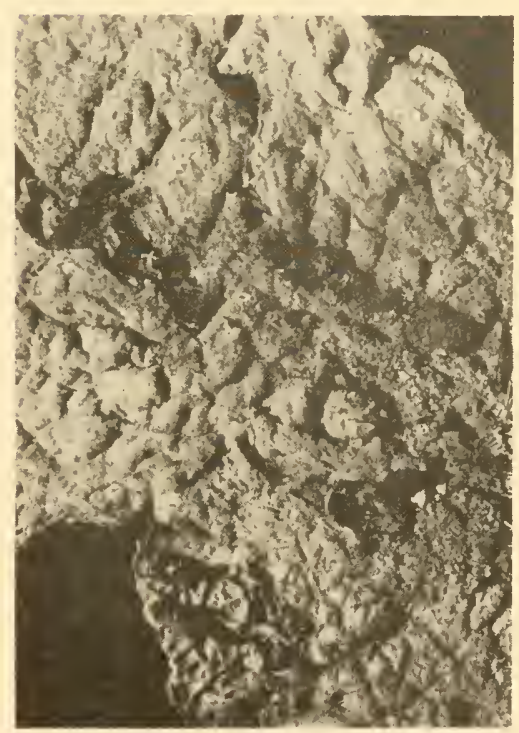

D 


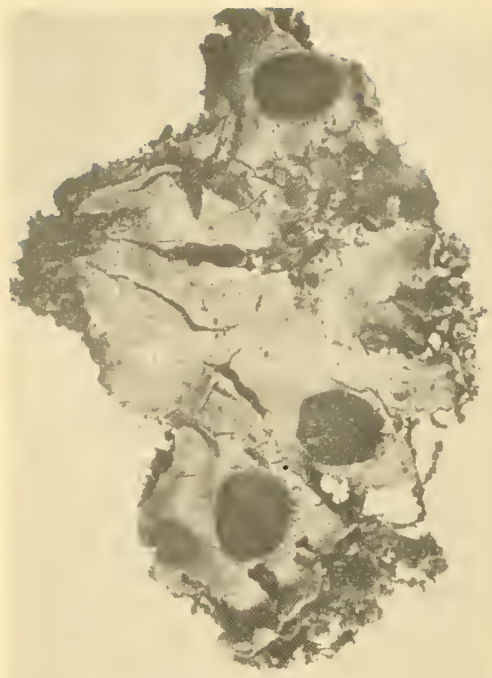

A

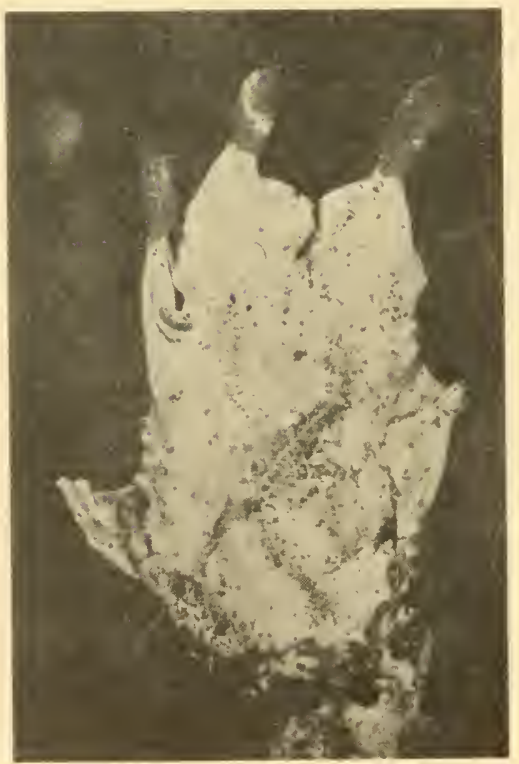

C

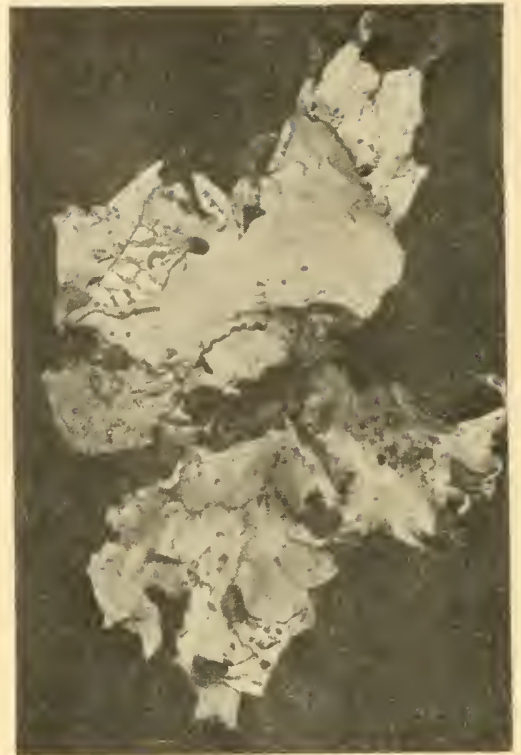

B

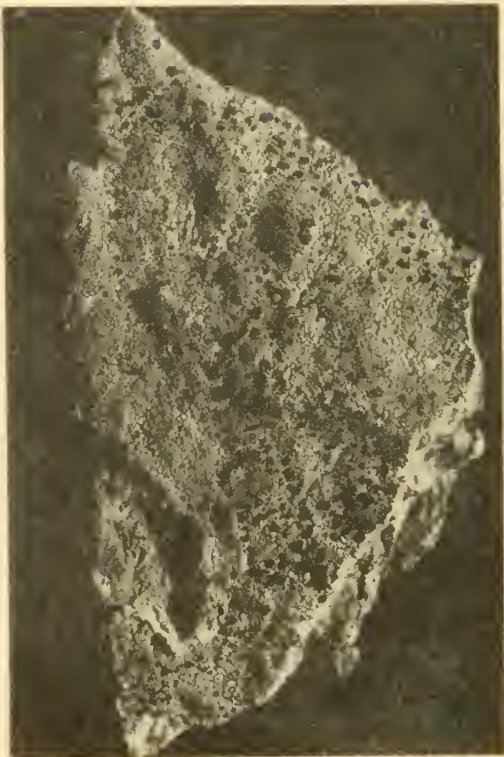

D 


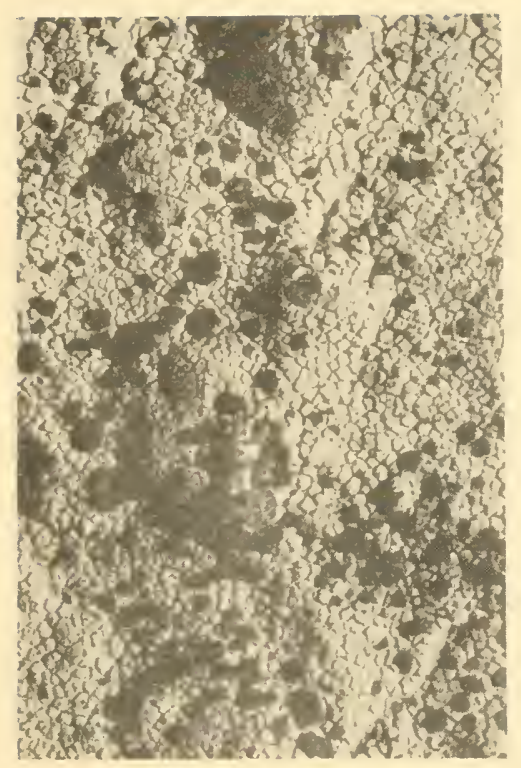

A

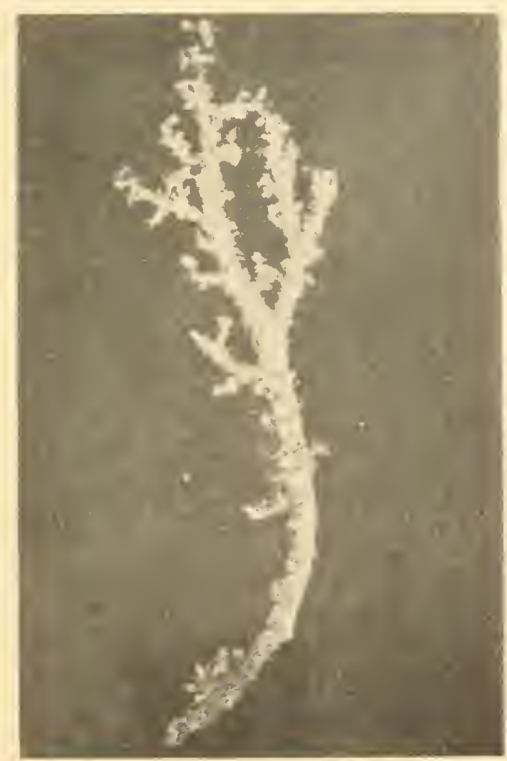

C

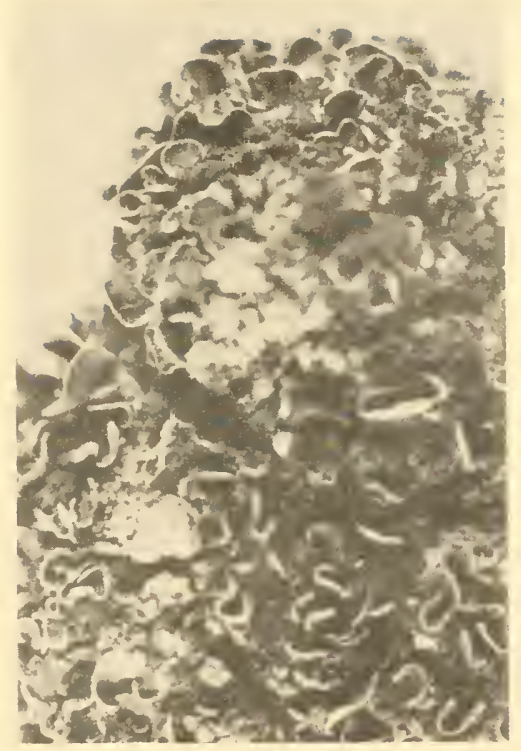

B

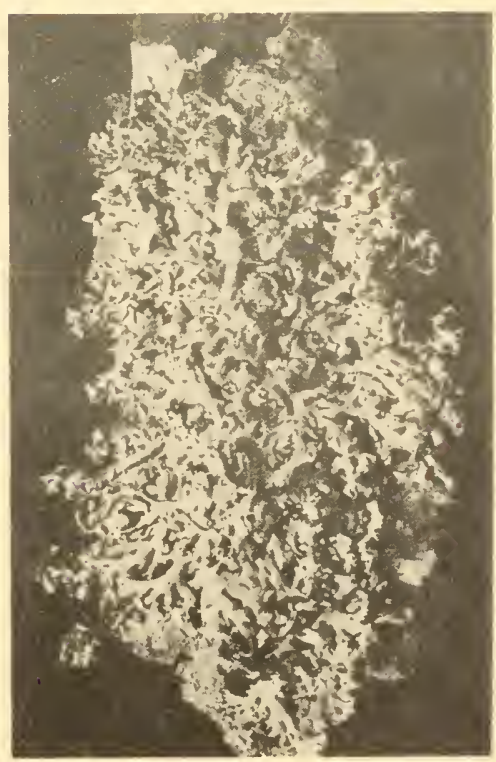

D 


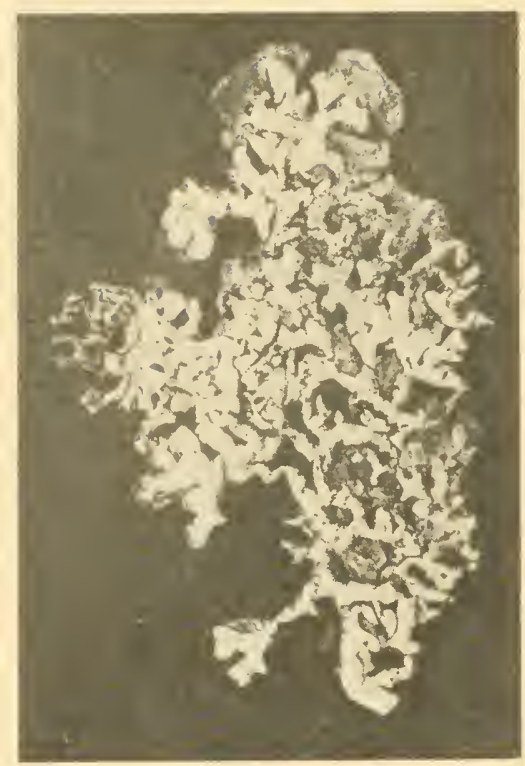

A

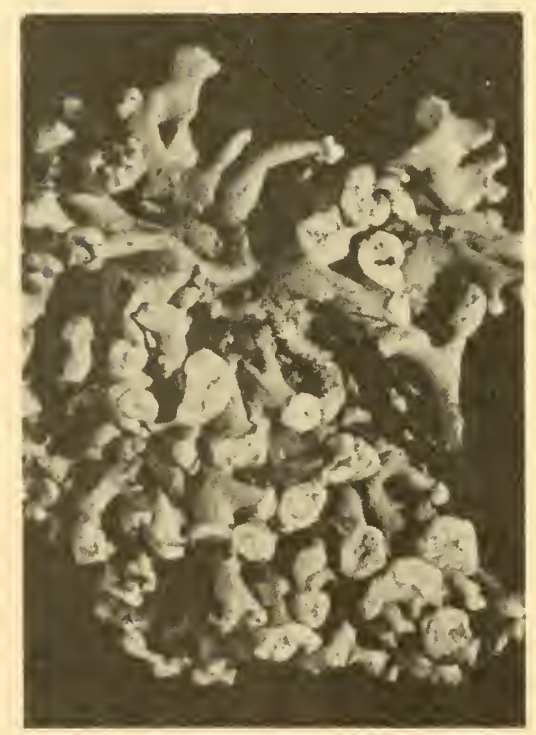

C

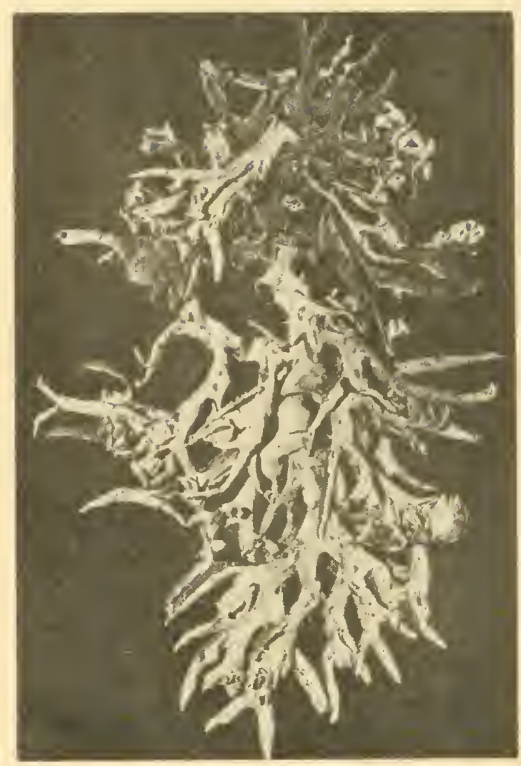

B

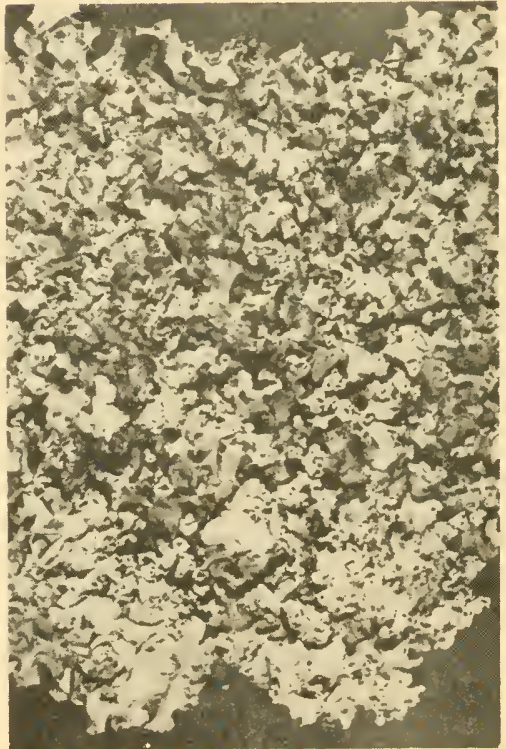

D

Plate V 


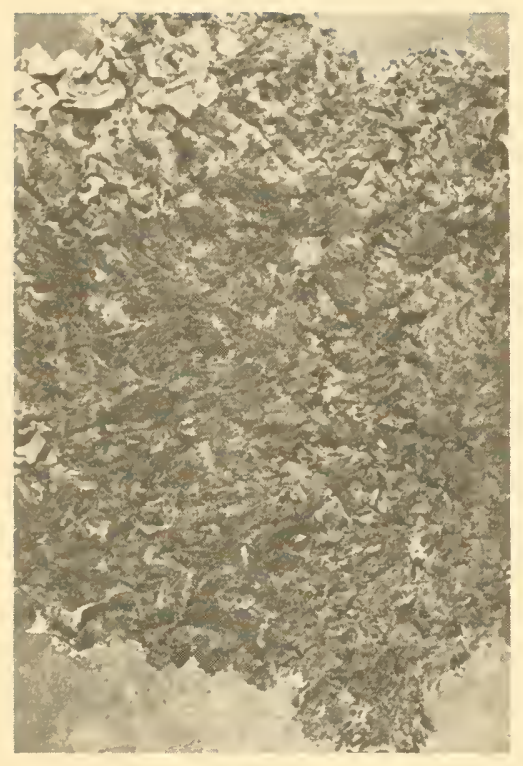

A

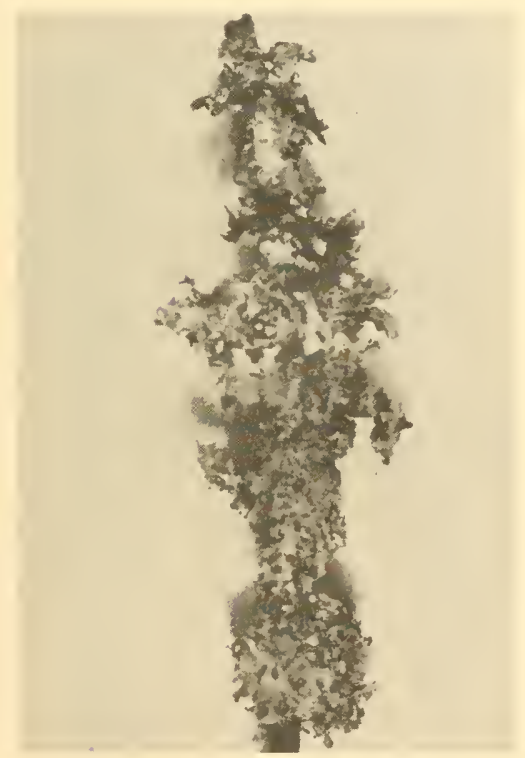

C

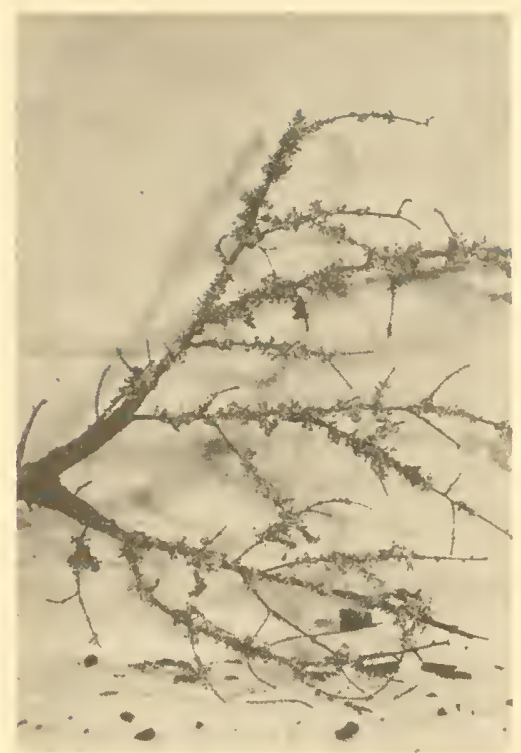

B

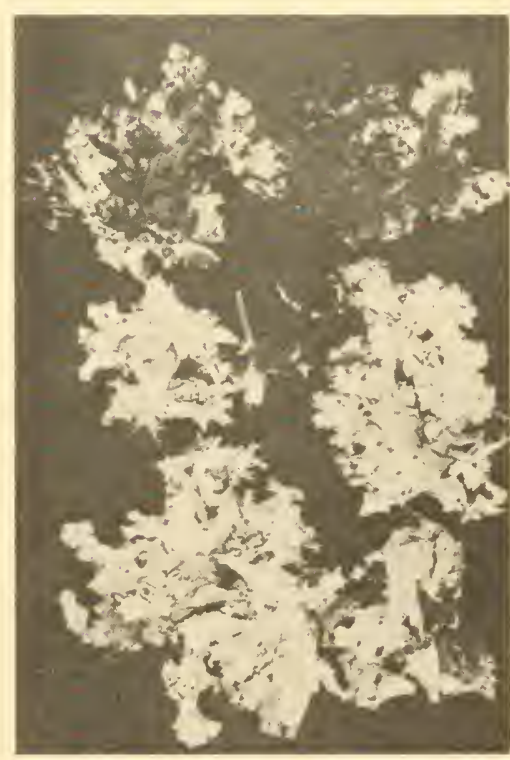

D

Plate II 


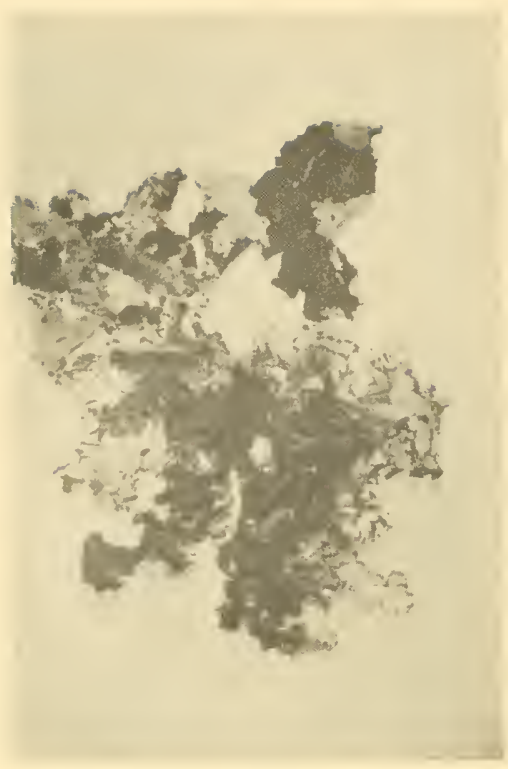

A

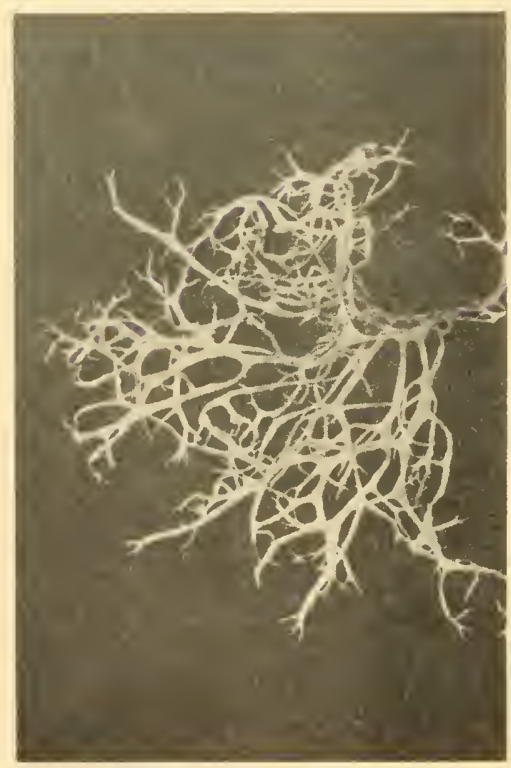

C

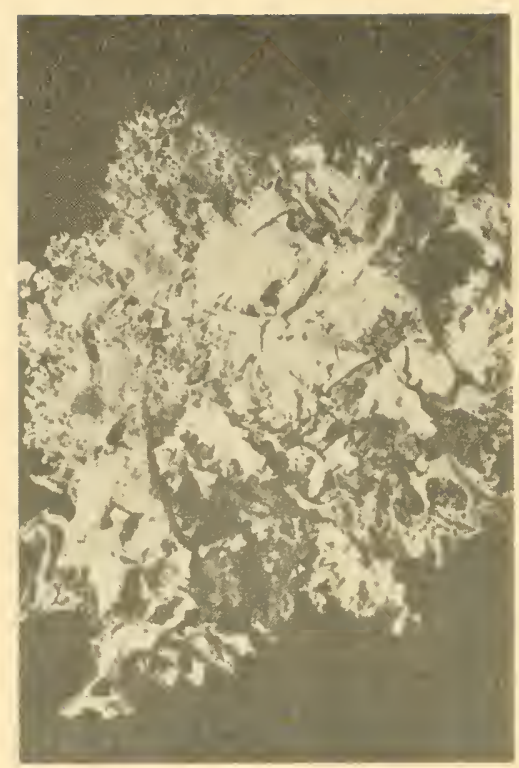

B

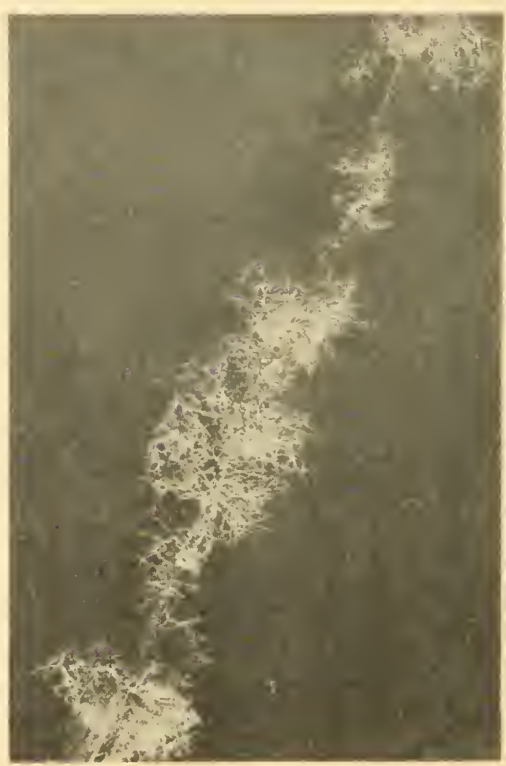

D

Plate VII 


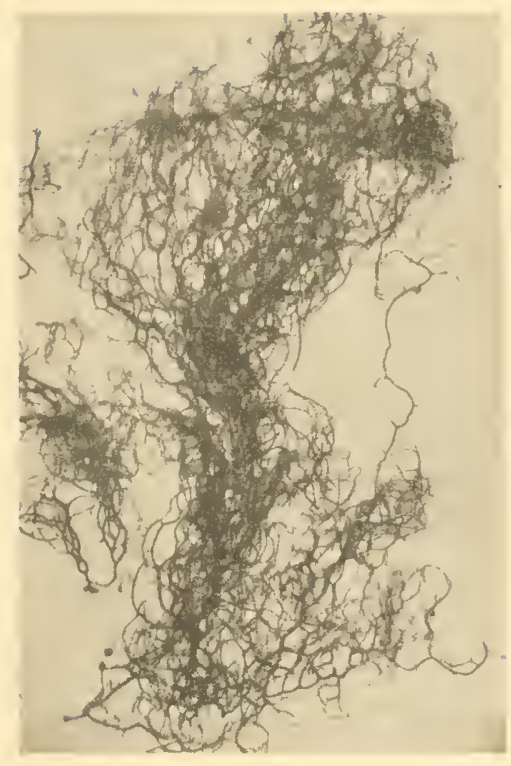

A

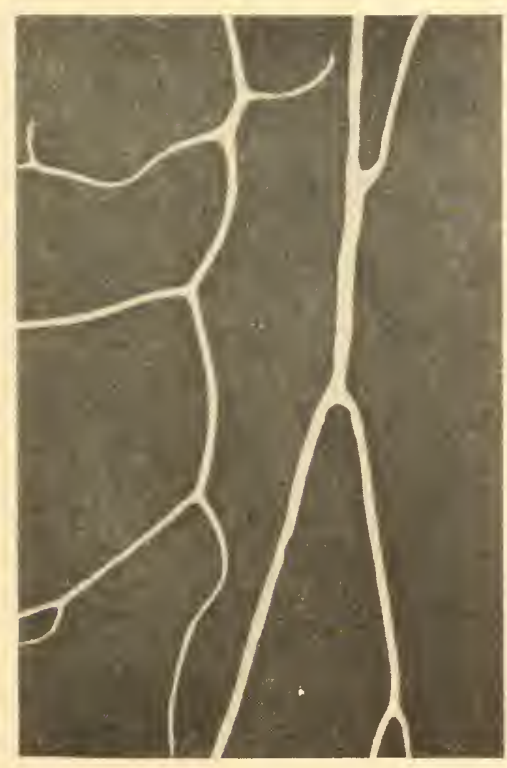

C

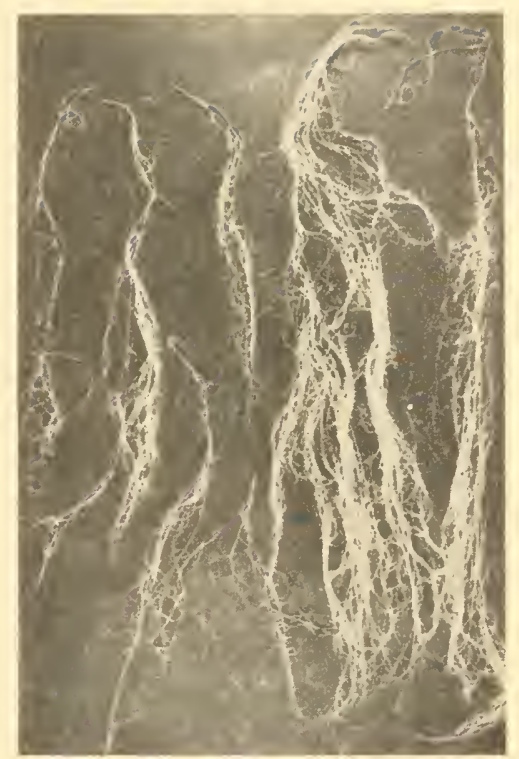

B

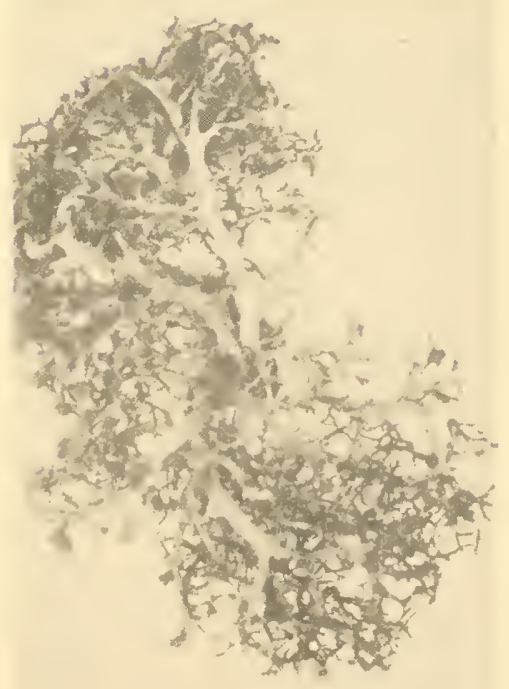

D 


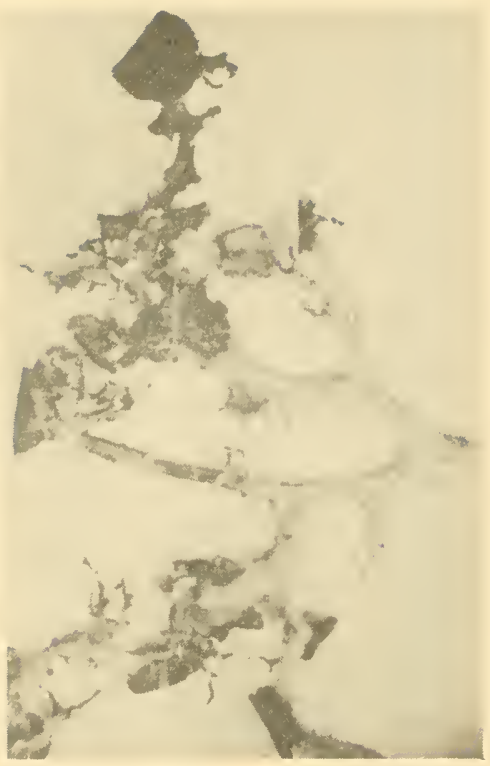

A

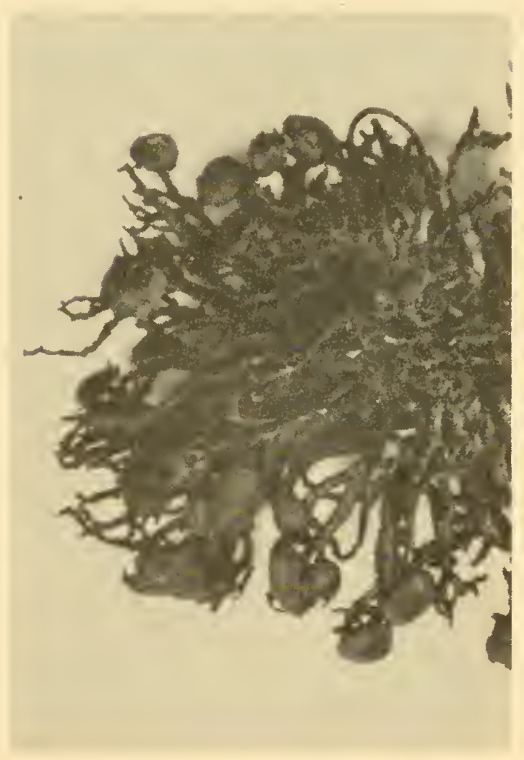

C

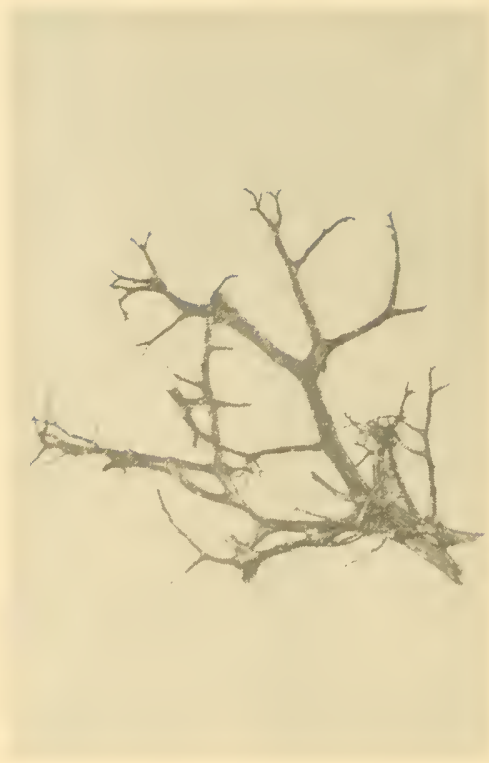

B

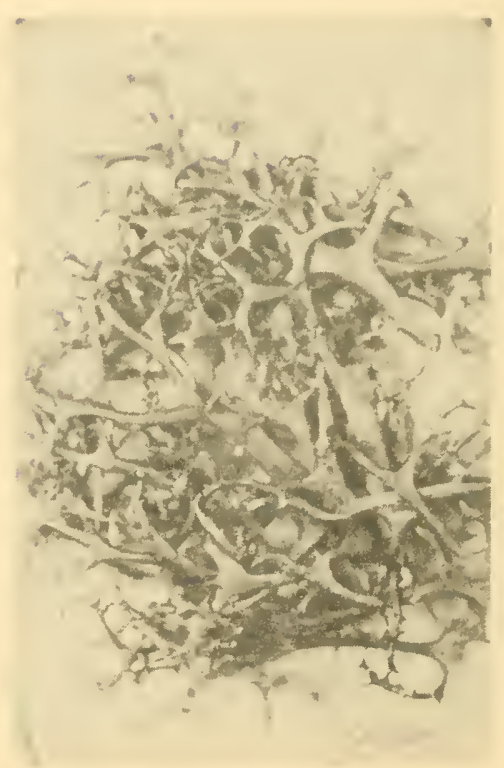

D 


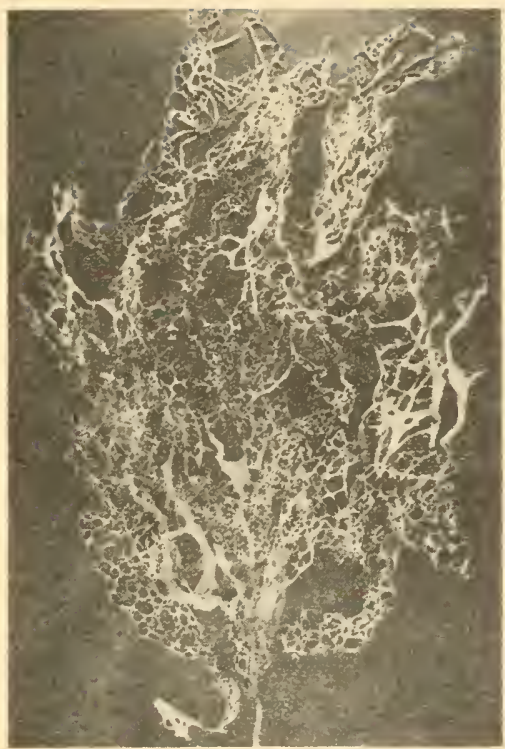

A

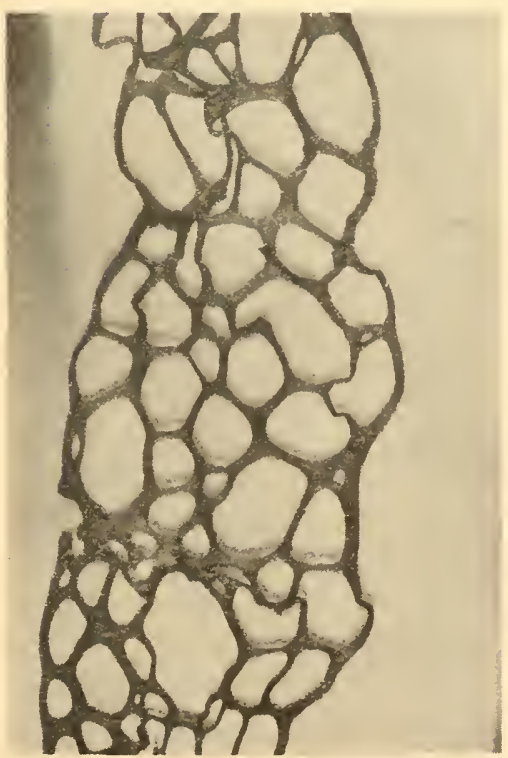

C

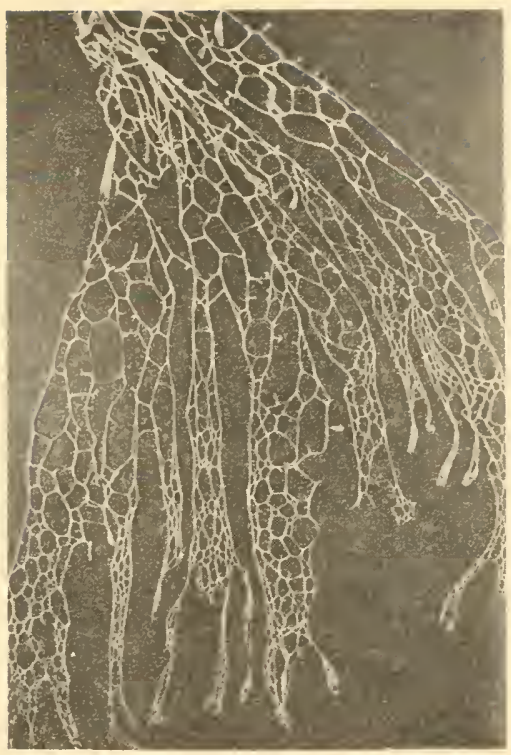

B

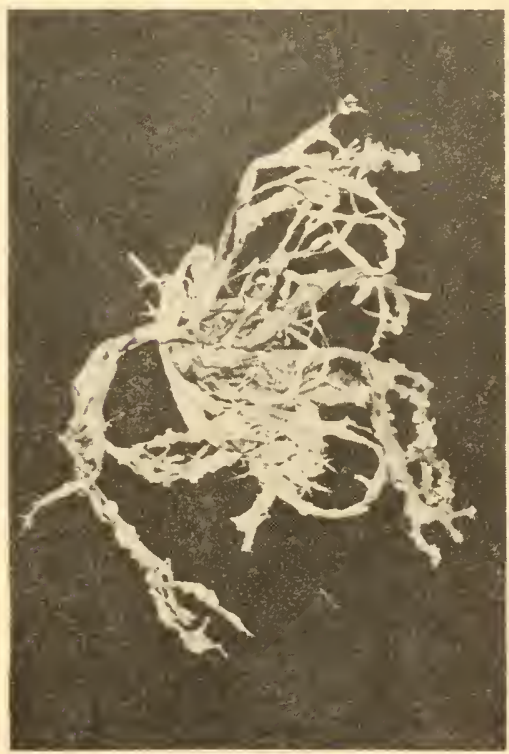

D 


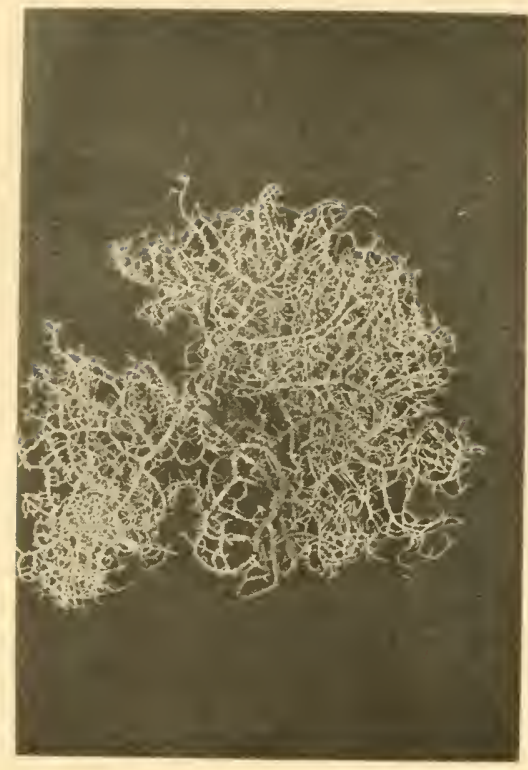

A

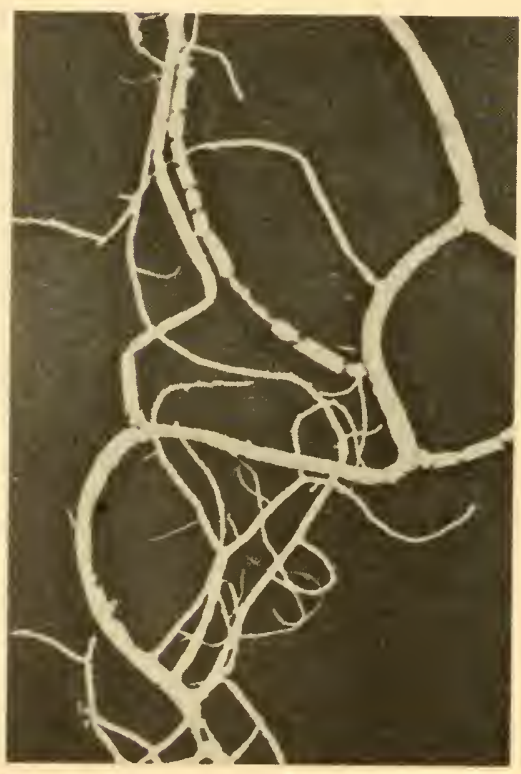

C

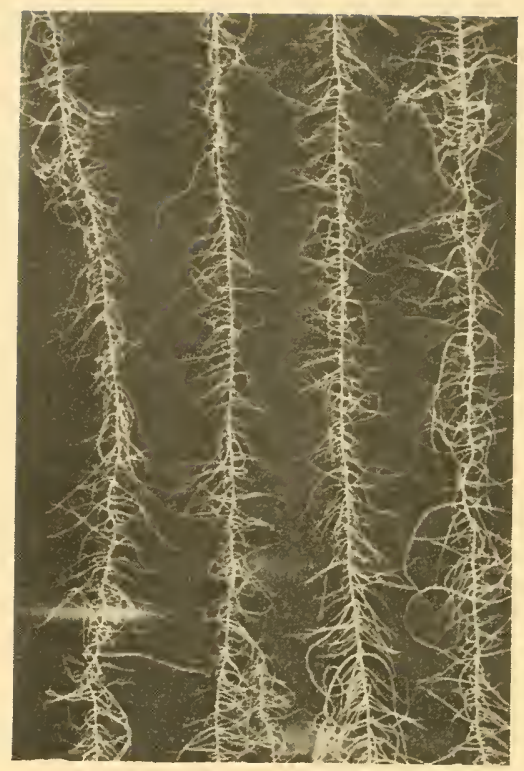

B

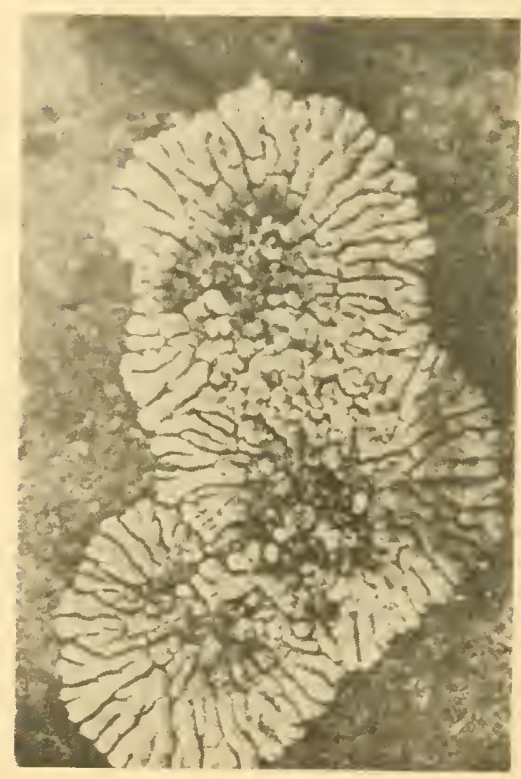

D 
Foster 2605 1/2 (W). San Juan Co.: San Juan Island, Friday Harbor, 1906, Fink (W). Clallam Co.: Lake Crescent, Storm King Mountain, 3800 ft., 1935, A. H. Smith 11130 (F).

This is an abundant Cladonia west of the Cascades; rather easily recognized by the cupless, corticate podetia, and the small brown disked apothecia borne on the tips of the branched podetia.

19. Cladonia furcata var. pinnata (Flk.) Wainio, Mongr. Clad. Univ. $1: 332.1887$.

Cenomyce racemosa var. pinnata Flk. in Schleich. Cat. Pl. Helv. 47. 1821.

Podetia abundantly covered with crenate to incised squamules, otherwise like the species.

On soil or decaying wood: Pacific Co.: Chinook, 1931, 802. Clallam Co.: Lake Sutherland, 590 ft., 1931, 1012. San Juan Co.: San Juan Island, Pt. Caution, 1931, 2070. King Co.: Richmond Highlands, $300 \mathrm{ft} ., 1931$, 578; Edgewood, 300 ft., 1931, 1826. Pierce Co. : Silver Springs, $2670 \mathrm{ft}$., 1931, 1119. San Juan Co.: Orcas Island, 1906, Fink 135 (W). Clallam Co.: Lake Crescent, Storm King Mountain, 3000 ft., 1935, A. H. Smith $2223(\mathrm{~F})$.

Zahlbruckner, Sandstede, Evans, and Fink all recognize other varieties and forms of Cladonia furcata (Huds.) Schrad. However, I will follow my policy established in the treatment of the Cladonias, and not divide the species Cladonia furcata any further.

20. Cladonia gracilis (L.) Willd. Fl. Berol. Prodro. 363. 1787.

Lichen gracilis Linn. Sp. P1. 1152. 1753.

Primary thallus persistent or sometimes disappearing, greenish gray or olive-buff $(\mathrm{R})$, consisting of medium sized, rather broad, crenate margined squamules, flat to ascending, underside white or creamy. Podetia gray, grayish olive $(R)$, brownish olive $(R)$, or reddish brown, in clusters, but not entangled, erect or ascending, cylindrical, simple or branched, sometimes dying at the base, cortex is often continuous, at times consisting of contiguous or scattered areoles; cups are commonly present, shallow, varying in width, margins are dentate or proliferate, rarely proliferating from the center, often proliferations are cup bearing, or cups are entirely absent, replaced by subulate tips. Apothecia sessile or with a short stalk on the margin of the cup, often confluent; disk pale or dark brown. $\mathrm{KOH}+$ for some specimens, - for other specimens; $\mathrm{P}+$, bright orange pink.

On soil, among mosses, or on decaying wood: This species not collected by the writer. San Juan Co.: San Juan Island, Friday Harbor, 1906, Fink 65 (W). Clallam Co.: Elwha River above upper bridge, 1914, Foster 2771 (W); this specimen determined as Cl. gracilis var. cordalis. Port Angeles, Bird's, 1800 ft., 1914, Foster 2607 (F).

The smooth continuous cortex on the podetia, lack of soredia, and often 
cup bearing proliferations from the margins of the cups, help to distinguish this large and comprehensive species.

21. Cladonia gracilis var. chordalis (Flk.) Schaer. Lich. Helv. Spic. 32. 1823.

Capitularia gracilis var. cordalis Flk. Ges. Naturf. Freund. Mag. 4:264. 1810.

Podetia cupless, terminating in a sharp point, or with narrow cups, surface smooth, squamules very rare; otherwise like the species.

On soil: King Co.: Snow Lake Trail, 3500 ft., 1931, 1965. Pierce Co.: Mt. Rainier, Yakima Park, 6300 ft., 1931, 600; Berkeley Park, 6000 ft., 1940, 3013. Clallam Co.: Bogachiel Ridge, 5500 ft., 1935, A. H. Smith 2719 (F).

22. Cladonia gracilis var. elongata (Jacq.) Flk. Beschr. Braunfr. Becherf. 330. 1810.

Lichen elongatus Jacq. Misc. II, 368, 1781.

Podetia very long, tapering from the base, often cupless, sometimes with narrow cups; otherwise like the species.

On soil: Pierce Co.: White River Camp, 3000 ft., 1928, 1958; Mt. Rainier, Van Trump Park, 5000 ft., 1942, 3623. Clallam Co.: Olympic Hot Springs, 3500 ft., 1914, Foster 2874 (W). Mason Co.: Mt. Elinor, 3500 ft., 1912, Foster 2114 (W). Clallam Co.: Olympic Hot Springs, 1935, A. H. Smith 2117 (F).

\section{Cladonia macilenta Hoffm. Deutschl. Fl. 2 :126. 1796.}

Primary thallus rarely dying, grayish green, grayish olive $(R)$, or pale brown, consisting of crenate or lacinate-lobed squamules, strongly ascending, involute, underside white, sometimes sorediate. Podetia whitish, grayish green, or grayish olive $(R)$, often rather short and stout, somewhat club-shaped, or rarely rather slender, mostly decorticate and farinosesorediate, sometimes corticate, often squamulose near the base, at times corticate just below the apothecia ; cupless. Apothecia solitary or clustered on the tips of the podetia, often absent, small to medium sized, 0.5-2.0 mm. across; disk scarlet, irregularly convex. $\mathrm{KOH}+, \mathrm{P}+$, bright yellow orange.

On soil, decaying wood, often on charred wood: Pacific Co.: Oysterville, 1931, 753. San Juan Co.: San Juan Island, Pt. Caution, 1930, 1979. Island Co.: Whidbey Island, 1931, 1773. Kitsap Co.: Hidden Ranch near Bremerton, 213 ft., 1931, 1759. King Co.: Edgewood, 300 ft., 1931, 1811. San Juan Co.: San Juan Island, Friday Harbor, 1906, Fink 223 (W). Thurston Co.: Rochester, 1912, Foster 2074 (W). Clallam Co.: Cape Flattery, 1935, A. H. Smith 1896 (F) ; Lake Crescent, 1935, A. H. Smith 1954 (F).

The rather commonly sterile apices of the podetia distinguish this Cladonia. 
24. Cladonia multiformis Merrill, Bryol. 11:110. 1908.

Cladonia furcata var. Finkii Wainio.

Cladonia palamaea (Ach.) Fink n. comb.

Primary thallus soon dying, only seen in young plants, greenish gray or dark olive-buff $(\mathrm{R})$, consisting of small to medium, elongated, narrow lobed squamules with crenate to sinuate margins, underside white. Podetia whitish, pale greenish gray, or olive-buff $(R)$, usually growing close together, well developed, cylindrical to flattened, irregularly swollen, sometimes fissured and gaping, simple or sparingly rather short branched, cortex continuous or areolate, densely squamulose throughout, tips sharp pointed, truncate, or cupbearing; cups broad and conspicuous, closed by membranes that are lacerated or perforated in various ways, no other Cladonia so far as known has closing membranes like these, margins of cups more or less dentate, or repeatedly proliferate, proliferations are cupforming or may become variously branched. Apothecia small and inconspicuous, solitary or clustered on the margins of cups or on the tips of the branches; disk small, brown. $\mathrm{KOH}-\mathrm{P}+$, reddish orange.

On soil or rock: Clallam Co.: Cedarville, 1940, 2895. San Juan Co.: San Juan sland, Trout Lake, 1940, 3231; Orcas Island, Mt. Constitution, 2500 ft., 1928, 2071. Kitsap Co.: Hidden Ranch, near Bremerton, 213 ft., 1930, 526. Yakima Co.: Little Naches, 2557 ft., 1931, 1003. Pend Oreille Co.: Metaline Falls, 2600 ft., 1931, 1479 (F). Ferry Co.: Republic, 1912, Foster 2311, 2357 (F).

This species grows in great abundance in parts of the state; it is easily identified by the broad open cups that are closed by peculiar lacerated or perforated membranes, and by the fissured and gaping podetia.

25. Cladonia nemoxyna (Ach.) Nyl. Lich. Novae Zelandiae 18. 1888.

Baeomyces radiatus. B. nemoxymus Ach. Meth. Lich. 342. 1803.

Cladonia fimbriata var. nemoxyna (Ach.) Wainio.

Primary thallus usually persistent, whitish, cream-buff $(R)$, olive-buff (R), or light brown, consisting of small to medium sized, digitately lobed squamules, flat to ascending, underside white, sorediate. Podetia concolorous with the primary thallus, clustered, erect, usually long and slender, lower part more or less corticate, upper decorticate, granular-sorediate; cups narrow and shallow, some times only abortive cups appear, at times the tips of the podetia are cupless, margins are entire, dentate, or proliferate, cups often sorediate inside. Apothecia rather small and inconspicuous, sessile or with a short stalk on the margin of the cups; disk brown or pale reddish brown. $\mathrm{KOH}-\mathrm{P}+$, dull orange.

On soil and decaying wood: Clallam Co.: Port Angeles, Bird's, 1800 ft., 1911, Foster 2607 (W). Grays Harbor Co.: Copalis, 1911, Foster $1490 f$ (W). San Juan Co.: San Juan Island, Friday Harbor, 1906, Fink 114 (W). King Co.: Kent, G. N. Jones $3048 a$ (W). 
The shallow narrow cups on long slender podetia, with the upper part usually grandular-sorediate, characterize this species.

26. Cladonia polydactyla (Flk.) Wainio, Monogr. Clad. Univ. $1: 119.1887$.

Cenomyce polydactyla Flk. Deutsch. Lich. 10:13. 1815.

Cladonia flabelliformis (Flk.) Wainio.

Primary thallus persistent, chamois $(R)$, cream-buff $(R)$, brown, or pale green, consisting of medium to large, crenate, lobate or irregularly dissected squamules, flat to erect, margins somewhat involute, underside white, sparingly sorediate. Podetia concolorous with the primary thallus, solitary or clustered, slender, simple or rarely branched, cylindrical, corticate near the base, rarely squamulose, upper part corticate, sorediate; cups narrow, gradually dilated, shallow, margins lacerate, dentate, or subentire, and sometimes once or twice proliferate. Apothecia on short stalks on the margins of the cups, solitary or confluent, small to medium sized, 0.5$2.5 \mathrm{~mm}$. across; disk scarlet, convex. $\mathrm{KOH}+, \mathrm{P}+$, orange red.

On soil or old wood: Clallam Co.: near Elwha Bridge, 1940, 2886. Pacific Co.: Loomis Lake, 1931, 765. Thurston Co.: Grand Mound, 1940. 2947. King Co.: Edgewood, 300 ft., 1931, 1976.

Rather a common bark lichen in the state; the large persistent primary squamules help to identify it.

27. Cladonia pyxidata (L.) Hoffm. Deutschl. F1. 2:121. 1795.

Lichen pyxidata Linn. Sp. P1. 1151. 1753.

Primary thallus usually persistent, greenish gray or olive-buff $(R)$, consisting of medium sized, concave or convex, crenate or digitately lobed squamules, ascending, underside white or yellowish, sorediate. Podetia concolorous with the primary squamules, rather short, erect, solitary or clustered, unbranched, cupbearing ; cups deep goblet form, gradually widening from the base, margins dentate or proliferate, cortex smooth and contiguous, or warty and areolate near the base, sometimes decorticate and sorediate near the top, rarely squamulose anywhere, inside of the cups often lined with convex verruculae. Apothecia rare, when present, sessile or borne on short stipes on the margins of the cups; disk brown. $\mathrm{KOH}$-, $\mathrm{P}+$, bright orange or red.

On soil or decaying wood: Clallam Co.: Lake Sutherland, $490 \mathrm{ft}$., 1931, 1011. Pierce Co.: Mt. Rainier, Moraine Park Trail, 3300 ft., 1940, 3002. Kittitas Co.: Easton, 2168 ft., 1931, 854. Jefferson Co.: Mt. Olympus, near Humes Glacier, 5500 ft., 1907, T. C. Frye 84 (W). San Juan Co.: San Juan Island, Friday Harbor, 1906, Fink 70 (W).

$\mathrm{Cl}$. chlorophaea and $\mathrm{Cl}$. pyxidata both have deep goblet form cups and a positive reaction with paraphenylenediamine; however, $\mathrm{Cl}$. pyxidata has a smooth cortex, sometimes areolate near the base of the cups, the upper part is sometimes decorticate and sorediate; while $\mathrm{Cl}$. chlorophaea has soredia all over the surface of the cups, both inside and out. 
28. Cladonia pyxidata var. pocillum (Ach.) Flot. Linnaea 17:19. 1843.

Baeomyces pocillum Ach. Meth. Lich. 336. 1803.

Primary thallus consisting of thick, round lobed, adnate, rarely ascending, squamules, crowded, forming a pale brown or greenish brown crust, usually sterile; otherwise like the species.

On soil: Clallam Co.: Lake Sutherland, 490 ft., 1931, 1017. King Co. : Edgewood, 300 ft., 1931, 1821. Pierce Co.: Mt. Rainier, Van Trump Park, $5000 \mathrm{ft} ., 1942,3618$.

29. Cladonia squamosa (Scop.) Hoffm. Deutschl. Fl. 2:125. 1796.

Lichen squamosus Scop. Fl. Carn. 2nd ed., 2:368. 1772.

Primary thallus usually persistent, light brownish olive $(\mathrm{R})$ or greenish gray, consisting of medium sized, circular, crenate to sinuate, squamules, flat to ascending, underside white. Podetia about the same color as the primary thallus, clustered, cylindrical, erect or ascending, flexuous, irregularly to radiately branched, cortex has a tendency to disintegrate, often becoming areolate toward the base, sorediate on the upper part, usually squamulose, axils often open, with or without cups; cups may be wide open to almost closed, margins entire to repeatedly proliferate. Apothecia small, usually clustered on the margins of the cups, or borne on the tips of the proliferations, or on the tips of the branches; disks pale or darker brown. $\mathrm{KOH}-, \mathrm{P}+$, pale yellow or ochraceous-buff ( $\mathrm{R})$.

On soil, on thin layer of soil over rocks, on rocks, or on old wood: San Juan Co.: San Juan Island, Friday Harbor, 1940, 3253. Clallam Co.: Olympic Hot Springs, 1935, A. H. Smith 2256 (F). Skagit Co.: Anacortes, 1906, T. C. Frye (W).

The negative reaction with $\mathrm{KOH}$ readily separates this species from Cl. subsquamosa.

30. Cladonia subsquamosa (Nyl.) Wainio Monogr. Clad. Univ. 1: 445. 1887.

Cladonia delicata var. subsquamosa Nyl. in Leight. Not. Lichenol. $11: 407.1866$.

Primary thallus not much in evidence, soon disappearing, greenish gray or brownish-olive ( $R$ ), consisting of small to medium sized, oblong, deeply lobed squamules, with irregular margins, underside white. Podetia about the same color as the primary thallus, sometimes a little lighter, often crowded, erect, slender, subcylindrical or cylindrical, usually hollow, simple or irregularly branched, axils sometimes perforate, often dying at the base, cortex rough, warty, or areolate, or almost entirely lacking, squamulose only toward the base; cups when present, small, perforate, margins proliferate with short, slender, rough branches. Apothecia solitary or clustered on the tips of branches; disk small, brown. $\mathrm{KOH}+$, bright yellow, turning pinkish red, $\mathrm{P}+$, orange red. 
On soil, on thin layer of soil over rock, or on decaying wood: Pacific Co. : Oysterville, 1931, 761. San Juan Co.: San Juan Island, Friday Harbor, 1928, 1970. Kitsap Co. : Hidden Ranch, near Bremerton, 213 ft., 1931, 1756. King Co.: Chase Bog, 300 ft., 1940, 3345; Edgewood, 300 ft., 1940, 3080. San Juan Co. : Blakely Island, 1906, Fink 217 (W). Whatcom Co.: near Glacier, 1914, Foster (W). King Co.: Seattle, Ballard Beach, 1913, Foster (W). Clallam Co. : Lake Crescent, 1935, A. H. Smith 2256 (F).

A common species west of the Cascades; any part of this Cladonia when treated with $\mathrm{KOH}$ turns yellow and then pinkish red; this striking color change, combined with the abundant squamules on the podetia, make this an easy species to recognize.

31. Cladonia subsquamosa f. luxurians Nyl. in Leight. Not. Lichenol. 11 :407. 1866.

Podetia luxuriantly squamulose throughout their entire length. Apothecia abundant; disk pinkish brown or fawn color ( $R$ ) ; otherwise like the species.

On soil or decaying wood: King Co.: Edgewood, 300 ft., 1931, 1801. Clallam Co.: Port Angeles, Bird's, 1914, Foster 2606a (W), Foster 2606 (F).

32. Cladonia uncialis (L.) Web. in Wiggers, Prim. Fl. Hols. 90, No. 995. 1780.

Lichen uncialis Linn. Sp. P1. 1153. 1753.

Primary thallus greenish gray or yellowish green, consisting of small to medium sized squamules, brittle, soon disappearing, seldom seen. Podetia straw yellow $(R)$, yellowish green, or dark olive-buff $(R)$, cylindrical, dilated, dichotomously or radiately branched, sometimes fastigiated, crowded and irregularly clustered, forming large masses, often dying at the base, tips often dilated, normally sharp-pointed, may be rounded or truncate, axils often open, cortex continuous smooth and shiny or areolate, no squamules; cupless. Apothecia small, solitary or clustered on tips of short branches; disk brown. $\mathrm{KOH}-, \mathrm{P}-$, no color changes with either.

On soil: San Juan Co.: San Juan Island, Friday Harbor, 1928, 1954; Trout Lake, 1940, 3223; Orcas Island, Mt. Constitution, 2500 ft., 1928, 2076; Brown Island, 1906, Fink 107 (W) ; Orcas Island, Mt. Constitution, 2500 ft., 1906, Fink 313 (W). Clallam Co.: Lake Crescent, Storm King Mountain, 4000 ft., 1935, A. H. Smith 2215 (F).

This species is easily identified by the very brittle, much branched podetia, bearing very small apothecia on the tips and the early disappearance of the primary thallus. Both in the field and in the herbarium, the podetia are often found broken into pieces.

\section{Cladonia verticillata Hoffm. F1. 2:122. 1795.}

Primary thallus usually persistent, greenish gray or brown, consisting of medium sized or larger crenate or incised-lobate squamules, ascending, 
somewhat involute, clustered or scattered, underside white or cream colored. Podetia gray, greenish gray, dark olive-buff $(\mathrm{R})$, or light brown, cylindrical, erect, hollow, in small groups or solitary, cortex subcontinuous, or composed of contiguous areoles, rarely squamulose toward the base; cups abruptly dilated, shallow, $2-5$ ranked, cup bearing proliferations from the center of the cups, from the margins of the cups, or from the sides of the podetia. Apothecia sessile or on short stipes on the margins of the cups; disk brown, convex. $\mathrm{KOH}-, \mathrm{P}+$, brick red.

On soil, or sometimes on a thin layer of soil over rock: Kitsap Co.: Hidden Ranch, near Bremerton, 213 ft., 1931, 1723. Grays Harbor Co.: Pacific Beach, 1911, Foster 202 (F). Clallam Co.: Olympic Hot Springs, $1935, A$. H. Smith 2142 (F). This specimen determined as $\mathrm{Cl}$. verticellata var. cervicornis by J. Hedrick Jones.

34. Cladonia verticillata var. evoluta Th. Fr. Lich. Scand. 1 :83. 1871-1874.

Proliferations always from the center of the cup, forming a series of 4 or 5 cups one directly above the other; this outstanding arrangement sets this variety off from the species; otherwise it is like the species.

On soil: Kittitas Co.: Hidden Ranch near Bremerton, 213 ft., 1931, 1760. Grays Harbor Co.: Westport, 1908, Foster 711 (F). Spokane Co.: Deep Creek Canyon, T. A. Bonser (F).

\section{Stereocaulon Schreb. Gen. P1. 2 :768. 1791.}

Thallus of two kinds: a primary thallus, horizontal, crustose and warty, that soon disappears; no differentiation into layers; a permanent vertical thallus attached to the substratum by hyphal rhizoids. Podetia, which arise from the primary thallus, are erect, solid, cylindrical, branched, ecorticate, covered more or less thickly with granules or squamules. Apothecia terminal or lateral; proper margin soon disappearing; hypothecium hyaline; paraphyses unbranched and septate; asci usually 8-spored; spores hyaline, 3 -more-septate. Cephalodia containing various species of bluegreen algae are often present.

Alga Protococcus.

A. Podetia powdery-chalky; apothecia and cephalodia unknown.

1. S. albicans

A. Podetia not powdery-chalky; apothecia and cephalodia frequently seen.

B. Squamules palmate-digitate.

C. Podetia smooth or faintly tomentose; cephalodia containing Stigonema.

4. S. paschale

C. Podetia usually densely tomentose; cephalodia containing Nostoc.

D. Podetia medium in length, frequently branched, squamules abundant, crowded. .................................................. S. S. tomentosum

D. Podetia elongated, sparingly branched, squamules few, scattered. 6. S. tomentosum var. simplex 
B. Squamules not palmate-digitate.

E. Plant shrub-like, squamules umbilicate; cephalodia containing Stigonema. 3. S. denudatum

E. Plant not shrub-like, squamules coarsely granular; cephalodia containing Nostoc.

2. S. alpinum

1. Stereocaulon albicans Th. Fr. De Stereocaul. et Pilophor. Comment. 36. 1857.

Stereocaulon nanum Tuck. Stereocaulon tenellum Tuck.

Primary thallus present or soon disappearing. Podetia chalky-white, caespitose, short, usually less than $1 \mathrm{~cm}$. long, slender, fragile, branched, branches close together, intertwined, lower part of podetia smooth, upper part bearing greenish gray or gray granular squamules, that often become white powdery masses. Apothecia absent. $\mathrm{KOH}+$, yellow.

On soil: Chelan Co.: Merritt, 2186 ft., 1931, 1087a. Yakima Co. : Cliff near Naches Ranger Station, 2400 ft., 1940, 3329. Fink (1935) reports it from Washington.

This small and inconspicuous lichen is probably much more common in the state than the few stations reported indicate.

2. Stereocaulon alpinum Laur. in Funck, Cryptog. Gewächse 33. Heft 6. 1827.

Podetia grayish white or pale brown, stout, rather short, congested, erect or decumbent, frequently branched, tomentose, squamules appear as coarse crowded warty granules, on lower part of the podetia, becoming "incised crenate" Dodge (1929); cephalodia small, bluish green, greenish gray, or dark bluish glaucous ( $R$ ) when wet, grayish white when dry, rather conspicuous, called the spherical type by Johnson (1938), containing Nostoc. Apothecia rare, terminal or lateral, medium to large, 1.0-3.5 $\mathrm{mm}$. in diameter; disk reddish brown or brownish black, flat to convex, often swollen and breaking; proper margin concolorous with the disk, soon disappearing; spores hyaline, acicular, 3-septate, $20.0-37.0 \times 3.0-4.0 \mu$. $\mathrm{KOH}+$, yellow.

On soil or rocks: Whatcom Co.: $5500 \mathrm{ft}$, 1928, L. Sunquist 1834. Pierce Co.: Mt. Rainier, trail to Moraine Park, 3300 ft., 1940, 3000; Grand Park, 5575 ft., 1931, 1835; Berkeley Park, 6000 ft., 1940, 3019; Burroughs Mt., 7300 ft., 1931, 606. Okanogan Co. : Twisp Summit, 6060 ft., 1931, 1316.

A rather common alpine lichen, characterized by short congested podetia with coarsely granular squamules.

3. Stereocaulon denudatum Flk. Deutschl. Lich. part 4:13. 1819.

Podetia grayish white or some shade of brown, short to medium in length, solitary or densely crowded, erect or ascending, slender, shrub like, branching in the upper part of the podetia, lower part smooth, upper 
squamulose, squamules umbilicate, margins crenate or often covered with coarse rounded granules; cephalodia brown, gray, or black, the spherical type, Johnson (1938), containing Stigonema. Apothecia rare, terminal or lateral, small, 0.5-1.0 mm. in diameter; disk reddish brown or brownish black, flat to convex; proper margin concolorous with the disk, soon disappearing; spores hyaline, acicular, 3-5-septate, 20.0-38.0 $\times 2.5-4.0 \mu$. $\mathrm{KOH}+$, yellow.

On soil: Whatcom Co. : Ruth Mountain, 5500 ft., 1928, 1833. Washington Territory, Suksdorf (F). Washington Territory, Suksdorf (S).

Rather a rare alpine lichen.

\section{Stereocaulon paschale (L.) Hoffm. Deutschl. F1. 130. 1796.}

Lichen paschalis Linn. Sp. P1. 1153. 1753.

Podetia greenish white, greenish gray, or various shades of pale brown, short to medium in length, caespitose, usually spreading or decumbent, rarely erect, branched, glabrous or somewhat tomentose, squamules granulose or with short crenate marginal branchlets, palmate-digitate Riddle (1910) ; cephalodia gray, brown, or olivaceous, the spherical type, Johnson (1938), containing Stigonema. Apothecia terminal or sublateral, small, 0.5-1.5 mm. in diameter, often clustered; disk reddish black or brownish black; proper margin concolorous with the disk, thin, soon disappearing; spores hyaline, acicular, 3-septate, 25.0-35.0 × $3.3 \mu$. $\mathrm{KOH}+$, yellow.

On soil and rocks: San Juan Co. : Orcas Island, Mt. Constitution, 2500 ft., 1928, 2021. Kitsap Co.: Hidden Ranch, near Bremerton, 213 ft., 1931, 1724. Olympic Mountains, 1915, J. M. Grant 3 (F). Clallam Co.: Sequim, 1920, J. M. Grant (F). Columbia National Forest, R. Kienholz (F). Spokane Co.: Spokane River, 1908, T. A. Bonser (W).

The palmate-digitate squamules on the smooth or faintly tomentose podetia help to characterize this species.

5. Stereocaulon tomentosum Fries, Sched. Critic. Fasc. $3: 20.1824$.

Podetia grayish white or some shade of pale brown, medium in length to rather long, not often exceeding $8 \mathrm{~cm}$., stout, attached loosely to the substratum, erect or spreading, much branched, especially towards the top, densely to moderately tomentose, squamules abundant, palmate-digitate, crowded towards the upper part; cephalodia gray or bluish gray, warty, containing Nostoc. Apothecia numerous, subterminal or lateral, small, 0.4-1.0 mm. in diameter, clustered; disk reddish brown or brown; proper margin concolorous with the disk, thin, soon disappearing; spores hyaline, acicular, 3-7-septate, 20.0-37.0 × 2.6-3.5 $\mu$. KOH+, yellow.

On thin layer of soil over rock: Kitsap Co.: Hidden Ranch, near Bremerton, 213 ft., 1930, 1981. Clallam Co. : foot of Mt. Angeles, 1911, Foster 1778 (W) ; Olympic Hot Springs, 1935, A. H. Smith 2108 (F). Whatcom Co.: Boulder Creek Trail to Mt. Baker, $1000 \mathrm{ft} ., 1908$, C. S. Eaton 674 
(F). Thurston Co.: Gate, 1912, Foster 1947 (W). Spokane Co.: Spokane, 1930, Sister Marion Loretta (WTU).

The densely tomentose podetia, with cephalodia containing Nostoc, distinguish this species from $S t$. paschale, which it resembles rather closely.

6. Stereocaulon tomentosum var. simplex Riddle, Bot. Gaz. 50: 298, 1910.

Podetia elongated exceeding $8 \mathrm{~cm}$., slender, seldom branching, squamules few and scattered, otherwise like the species.

On soil : Mt. Rainier, Longmire Springs, 1904, T. C. Frye (F). Olympic Mountains, 1907, T. C. Frye (F).

\section{UMBILICARIACEAE}

Thallus foliose, 1-several-leaved, dorsi-ventral, with both upper and lower plectenchymacous cortices, also well-developed spongy algal and medullary layers, firmly attached to the substratum by a single umbilicus or by several cords. Apothecia circular to somewhat angular, scattered over the upper surface, immersed, adnate, sessile, or with a short stipe, the proper margin usually black, thalline margin seldom present; disk rarely smooth, usually gyrose-plicate; asci 1-8-spored; spores hyaline or brownish, nonseptate, 1-septate, or muriform.

Alga Protococcus.

42. Umbilicaria (Hoffm.) Nyl. em. Frey, Kaben-Krypt. Fl. 94 :205. 1933.

Gyrophora Ach. Meth. Lich. 100. 1803.

Thallus foliose, upper surface smooth or areolate cracked, often with squamules or with leaf-like appendages, with raised ridges; below smooth, papillate, warty, lacerate, with or without rhizoids, more or less fibrillose or hirsute, firmly attached to the substratum by a single umbilicus or by several cords. Apothecia scattered on the upper surface, circular or angular, immersed, adnate, or sessile, usually with a short stipe; proper margin prominent, thalline margin absent, hypothecium brownish or black; paraphyses more or less distinct; asci 1-8-spored; spores hyaline or finally brown, nonseptate or muriform.

A. Thallus with fibrils below.

B. Fibrils not always abundant.

C. Upper surface reticulated with black indented lines; below radiately ridged. 4. U. erosa

C. Upper surface not always reticulated, sometimes papillose; below not radiately ridged.

B. Fibrils always abundant.

10. U. virginis

D. Thallus large, upper surface gray, grayish brown, or black.

9. U. vellea

D. Thallus small to medium sized, dark brown, purplish brown or black.

E. Apothecia large, dome-shaped.

8. U. polyrrhiza 
E. Apothecia angulate or stellate.

1. U. angulata

A. Thallus with no fibrils below.

F. Upper surface smooth.

G. Thallus several-leaved (polyphyllous), black..........7. U. polyphylla

G. Thallus usually one-leaved, not black, but some shade of brown.

6. U. phaea

F. Upper surface not smooth.

H. Upper surface reticulate-ridged, covered with hard white granules.

2. U. decussata

H. Upper surface not reticulated.

I. Upper surface covered with a blackish scurf................ 3. U. deusta

I. Upper surface pustulose or papillose...................5. U. hyperborea

1. Umbilicaria angulata Tuck. Proc. Am. Acad. Arts and Sci. $1: 266.1848$. Gyrophora angulata (Tuck.) Herre, Cont. U.S. Nat. Herb. 13:318. 1911.

Thallus tawny brown, purplish brown, gray, or black with a slight bloom, small to medium sized, rarely large, one-leaved or at times manyleaved, smooth, thick and ridged, irregular in shape, margins crenate; below dark brown or black, rather densely granulose, much lacerated or ridged towards the center, bearing numerous rather pale fibrils. Apothecia rather rare, circular to angular, 1-3 mm. in diameter, adnate or subsessile; disk black, plicate, flat to convex; proper margin thick, persistent; hypothecium brown; asci 8-spored; spores hyaline, ellipsoid, nonseptate, 16.0$23.0 \times 7.0-14.0 \mu$.

On rock: Jefferson Co.: Olympic Mountains, foot of Humes Glacier, Queets River Valley, 5500 ft., 1907, Frye 42 (F). Fink (1919) reports it from the islands of Puget Sound.

2. Umbilicaria decussata (Vill.) Zahlbr. Cat. Lich. Univ. 8:490, 1932. Lichen decussatus Vill. Hist. P1. Dauphin 3 :964. 1789.

Gyrophora decussata (Vill.) Zahlbr.

Umbilicaria anthracina var. reticulata (Schaer.) Tuck.

Thallus grayish brown or blackish brown, small to medium sized, oneleaved, areolate, covered towards the center with reticulating ridges, the ridges and often the surface between them, whitened with hard granules, margins irregularly crenate; below smooth dark olive brown (R) or drab, lighter than the upper surface, smooth to minutely granular, without fibrils. Apothecia numerous, circular, 0.4-1.3 mm. in diameter, sessile; disk black rarely somewhat plicate, concave to convex; proper margin thick and persistent; hypothecium brown; asci 8-spored; spores hyaline, ellipsoid or ovoid, nonseptate, $11.0-15.0 \times 5.0-8.0 \mu$.

On rock: Pierce Co.: Mt. Rainier, Frying Pan River, 3500 ft., 1931, 678a. Columbia Co.: Blue Mountains, $6300 \mathrm{ft} ., 1931,1634$. Washington Territory, Cascade Mountains, Brandegee (F). 
This alpine species is easily recognized by the granules and ridges on the upper surface.

3. Umbilicaria deusta (L.) Baumg. Fl. Lipsiens. 571. 1790.

Lichen deustus Linn. Sp. P1. 1150. 1753.

Gyrophora deusta (L.) Ach.

Umbilicaria hyperborea var. deusta (L.) Tuck.

Umbilicaria flocculosa (Wulf.) Hoffm.

Thallus dull brownish black or fuscous-black ( $R$ ), small to medium sized, thin and fragile, easily broken, one-leaved or sometimes severalleaved, often deeply lobed, smooth or wrinkled, somewhat scurfy; below almost concolorous with the upper surface, smooth or somewhat pitted and lacunose, occasionally reticulately pitted and without fibrils. Apothecia very rare. I have seen no fruiting material from North America.

On rocks: San Juan Co.: San Juan Island, Friday Harbor, trail to Heaven, 1940, 3244. Pierce Co.: Mt. Rainier, Frying Pan River, 3500 ft., 1931, 678. Chelan Co.: Rainbow Trail, 3500 ft., 1931, 1279. Ferry Co.: Showers Butte near Republic, 1912, Foster 2305 (F).

This Umbilicaria has no very distinctive characters, except perhaps the thinness and fragileness of the thallus.

4. Umbilicaria erosa (Web.) Ach. Klg. Vetensk-Akad. Nya Handl. 15 :87. 1794.

Lichen erosus Web. Spicil. Fl. Goett. 259. 1778.

Gyrophora erosa (Web.) Ach.

Thallus dark reddish brown or dull dark brown, medium sized, oneleaved, rigid, surface wrinkled, reticulately cracked, these cracks often breaking entirely through the thallus, thus forming open spots; below pale brown or black, more or less radiately ridged, ridges broken and perforated, minutely granulose between the ridges, often bearing a few scattered fibrils. Apothecia numerous, circular, many angled, or variously shaped, 0.5-1.4 $\mathrm{mm}$. in diameter, adnate or subsessile; disk black, plicate, flat to convex; proper margin thin and finally disappearing; hypothecium brown; asci 8-spored; spores hyaline, ellipsoid, nonseptate, 6.6-9.0 x 4.5-7.0 $\mu$.

On rock: Whatcom Co. : Ruth Mountain, 6800 ft., 1928, 1877. Yakima Co.: American River Camp, 2800 ft., 1931, 947. Chelan Co.: White Rock Camp, 2200 ft., 1931, 1052b; Rainbow Trail, 3800 ft., 1931, 1273. Ferry Co.: Laurier, 1644 ft., 1931, 1420. Mason Co.: Mt. Elinor, 1912, Foster 2119 (F).

The open spaces in the thallus, combined with the numerous apothecia, make this a rather easy Umbilicaria to recognize.

5. Umbilicaria hyperborea (Ach.) Hoffm. Deutschl. Fl. 110. 1796.

Lichen hyperboreus Ach. Klg. Vetensk-Akad. Nya Handl. 15 :87. 1794.

Gyrophora hyperborea Ach. Meth. Lich. 104. 1803. 
Thallus chocolate, dark brown, or black, usually medium sized, sometimes small, one-leaved, round to irregular in shape, sparingly lobed, surface smooth, wrinkled, pustulose, or papillose, rarely perforate, margins torn and jagged; below pale or dark brown, or black with a grayish cast, smooth and somewhat pitted, without fibrils. Apothecia circular, angulate, or oblong, $0.5-2.0 \mathrm{~mm}$. in diameter, adnate or subsessile; disk black, plicate, flat to convex; proper margin thin; hypothecium dark brown; asci 8-spored; spores hyaline, ellipsoid, nonseptate, 10.0-13.2 x 5.0-6.6 $\mu$.

On rock: Pierce Co. : Mt. Rainier, Berkeley Park, 6000 ft., 1931, 650a; Burroughs Mountain, 7300 ft., 1931, 608. Chelan Co.: White Rock Camp, 2200 ft., 1931, 1052; Rainbow Trail, 3500 ft., 1931, 1248a. Okanogan Co.: Tonasket, 1200 ft., 1931, 1380. Ferry Co.: Mt. Gibraltar, 3100 ft., 1940, 3168. Lincoln Co.: Davenport, $2450 \mathrm{ft} ., 1931,1509$.

A common species, rather widely distributed east of the Cascades.

\section{Umbilicaria phaea Tuck. Lich. Calif. 15. 1866.}

Gyrophora phaea Nyl. in Hue Nour. Archiv. Mus. ser. 3. 3:37. 1891.

Thallus grayish brown or tawny brown, small or medium sized, sometimes very small, one-leaved, surface smooth; below darker in color, smooth without fibrils. Apothecia numerous, circular to slightly angular, 0.5-2.0 $\mathrm{mm}$. in diameter, immersed or adnate; disk black, plicate, convex; proper margin thin; hypothecium brown; asci 8-spored; spores hyaline or rarely brown, ellipsoid, nonseptate, 10.0-16.0 x 5.0-10.0 $\mu$.

On rock: Chelan Co.: Merritt, 2186 ft., 1931, 1086b. Yakima Co.: near Naches, Gleason's Ranch, 1350 ft., 1931, 900. Okanogan Co.: Tonasket, 1200 ft., 1931, 1365. Whitman Co.: Pullman, 2500 ft., 1931, 1583. Asotin Co.: Buffalo Rock, Snake River Canyon, 1927, G. N. Jones 3054 (W). San Juan Co.: Blakely Island, 1906, Fink 426 (W). Kittitas Co.: Vantage Bridge, 1942, P. F. Scholander (F).

Another species widely distributed east of the Cascades. The grayish brown color, combined with the numerous, rather crowded apothecia, help to identify this Umbilicaria.

7. Umbilicaria polyphylla (L.) Baumg. Fl. Lipsiens. 571. 1790.

Lichen polyphyllus Linn. Sp. P1. 1150. 1753.

Gyrophora polyphylla (L.) Funck.

Thallus dark brown or black, small or medium sized, many-leaved, very irregular in shape, deeply and irregularly lobed, margin crenate or dentate; below darker in color, almost always dull black, smooth or granulose, without fibrils. Apothecia very rare; I have seen no fruiting Washington specimen.

On rock: San Juan Co.: San Juan Island, Friday Harbor, 1928, 2024. Chelan Co.: White Rock Camp, 2200 ft., 1931, 1052a; Merritt, 2186 ft., 1931, 1086. Ferry Co.: Laurier, 1644 ft., 1931, 1421. Whitman Co.: Pullman, 1900, R. K. Beattie 46 (F). 
This many-leaved species is perhaps the most easily recognized of all species of Umbilicaria in the state.

8. Umbilicaria polyrrhiza (L.) Ach. Klg. Vetensk-Akad. Nya Handl. $15: 92.1794$.

Lichen polyrrhiza Linn. Sp. P1. 1151. 1753.

Gyrophora polyrrhiza (L.) Körb.

Thallus purplish brown or black, small or medium sized, one-leaved, becoming many-leaved, circular, margins crenate, somewhat torn; below black, becoming rather an olive color when moist, at times lacerate or ridged near the center, always densely covered with short black fibrils, that are rather knob-shaped at the apex; when there is a break in the surface these fibrils come through, forming strange black spots on the upper surface. Apothecia rather rare, large and dome-like, 2-5 $\mathrm{mm}$. in diameter, immersed at first, finally adnate; disk black, flat to gyrose-plicate; proper margin disappearing; hypothecium brown; asci 8-spored; spores hyaline, ellipsoid, nonseptate, 8.5-12.9 x 4.0-7.0 $\mu$.

On rock: San Juan Co.: Orcas Island, Mt. Constitution, $2500 \mathrm{ft} ., 1931$, 2066. Klickitat Co.: Goldendale, 2000 ft., 1931, 1696.

The densely fibrillose lower surface and the large dome-shaped apothecia characterize this species.

9. Umbilicaria vellea (L.) Ach. Kgl. Vetensk-Akad. Nya Handl. 15 :101. 1794.

Lichen velleus Linn. Sp. P1. 1150. 1753.

Gyrophora vellea (L.) Ach.

Thallus gray or grayish brown, somewhat white pruinose, large, oneleaved, usually round, becoming variously shaped, thick, rigid, surface smooth, or somewhat rough and cracked; below brown or black, with concolorous fibrils, very hirsute, sometimes lacerate near the center. Apothecia rare, circular, usually near the margin, $0.5-2.0 \mathrm{~mm}$. in diameter, adnate; disk black, plicate, convex; proper margin disappearing; hypothecium dark brown; asci 8-spored; spores hyaline, short-ellipsoid, nonseptate, 8.0-11.0 x 7.0-9.0 $\mu$.

On rock: Chelan Co.: Merritt, 2186 ft., 1931, 1095; White Rock Camp, 2200 ft., 1931, 1052; Rainbow Trail, 3800 ft., 1931, 1282. Ferry Co.: Showers Butte near Republic, 1912, Foster (W). Whitman Co.: near Pullman, 1913, J. S. Weaver 28 (F). Herre (1943) reports it from Mt. Baker, Heliotrope Ridge.

This species is easily identified by the large sized thallus and the very hirsute lower surface.

10. Umbilicaria virginis Schaer. Biblioth. Univers. Geneve, 36:135. 1841. Umbilicaria rugifera Nyl. Lich. Scand. 117. 1861.

Gyrophora rugifera. (Nyl.) Th. Fr. 
Thallus grayish brown, dark brown, olive brown, or black, small or medium sized, one-leafed, irregular in shape, rigid, rough, with coarse reticulated ridges, or papillose, with ridges not prominent ; below rosy gray, yellowish gray, or black, more or less covered with rather long pale or brown fibrils, occasionally these are reduced to papillae or very rarely the surface is smooth. Apothecia when present rather crowded, circular, 0.3-2.0 $\mathrm{mm}$. in diameter, adnate or sessile; disk black, not plicate, flat to convex; proper margin thin, permanent, flexuous; hypothecium brown; asci 8-spored, ventricose; spores rarely maturing, hyaline, ellipsoid, nonseptate, 7.0-15.0 × 5.0-9.9 $\mu$.

On rock: Columbia Co. : Blue Mountains, 6300 ft., 1931, 1635. Jefferson Co.: Summit of Mt. Olympus, 8200 ft., 1907, Frye 71 (W).

Found only at high elevations, easily recognized by the long abundant fibrils on the lower surface. 


\section{ACAROSPORACEAE}

Thallus crustose, squamulose, areolate, or subfruticose, occasionally marginally lobed, or entirely obsolete; with fairly complete differentiation into layers or all differentiation lacking, attached to the substratum by hyphal rhizoids or more rarely by an umbilicus. Apothecia circular or irregular in shape, scattered, immersed, adnate, or rarely stipitate, with either a proper or a thalline margin present; asci many-spored; spores very small, hyaline, nonseptate, or rarely 1 -septate, thin walled, with no gelatinous halo.

Alga Protococcus.

A. Thallus often obsolete; apothecia with proper margin.

43. Biatorella

A. Thallus present; apothecia immersed in it; apothecia with thalline margin. 44. Acarospora

43. Biatorella De Not. Giorn. Bot. Ital. $21: 192.1846$.

Thallus crustose, uniform or sublobate at the margins, often thin, scurfy, sometimes granular, evanescent, usually entirely obsolete, no differentiation into layers, attached to the substratum by hyphal rhizoids. Apothecia circular or angular, immersed, adnate, or sessile; proper margin present; hypothecium hyaline or brown; paraphyses fragile, thready, rarely branched, sometimes deliquescent; asci many-spored; spores very small, hyaline, nonseptate.

A. On soil; apothecia yellow or brown

5. B. fossarum

A. On rock or trees.

B. On trees, apothecia dark brown or black.

4. B. moriformis

B. On rock.

C. Thallus areolate on a black hypothallus

3. B. testudinea

C. Thallus often obsolete.

D. Disk pruinose, small.

1. B. pruinosa

D. Disk not pruinose, minute.

2. B. simplex

1. Biatorella pruinosa (Ach.) Mudd, Man. Brit. Lich. 191. 1861.

Lichen pruinosa Ach. Lichenogr. Suec. Prodro. 77. 1798.

Lecanora privigna var. pruinosa (Ach.) Tuck.

Biatorella simplex var. pruinosa (Ach.) Fink. 
Thallus grayish green, thin, and evanescent, sometimes a few granules scattered around the apothecia. Apothecia circular to somewhat angular, 0.5-2.0 mm. in diameter, adnate; disk dull black pruinose, when moist reddish black, flat; proper margin concolorous with the disk, entire or irregular, hypothecium yellow; spores hyaline, ellipsoid-oblong, nonseptate, $3.6-6.0 \times 1.0-2.0 \mu$.

On rock: Stevens Co.: Boyds, $1470 \mathrm{ft} ., 1931,1448$.

A rather inconspicuous rock lichen, perhaps the reason it has not been collected more often. However, the pruinose disk separates it very definitely from other Biatorellas.

2. Biatorella simplex (Davies) Branth \& Rostr. Bot. Tidssk. $3: 241.1869$.

Lichen simplex Davies Trans. Linn. Soc. London 2:283. 1793.

Lecanora privigna (Ach.) Nyl.

Thallus fine, scurfy, and soon evanescent. Apothecia circular or ver; irregular in shape, crowded or scattered, $0.2-0.5 \mathrm{~mm}$. in diameter, adnate or subsessile; disk brown or dull black, flat; proper margin concolorous with the disk, elevated, turgid; hypothecium hyaline or yellowish; spores hyaline, oblong-ellipsoid, nonseptate, 3.6-6.0 x 1.0-2.0 $\mu$.

On conglomerate rock or sandstone: Chelan Co.: Beaver Summit, 2800 ft., 1931, 1104. Cascade Mountains, 1925, J. M. Grant (WTU).

3. Biatorella testudinea (Ach.) Mass. Ricerch. Auton. Lich. 131. 1852. Lecidea cechumena var. testudinea Ach. Kgl. Vetensk.-Akad. Nya Handl. 232. 1808.

Lecidea morio Fries.

Thallus yellowish or copper color, somewhat shiny, crowded, flat, angular areoles, marginally lobed, on a black hypothallus, which makes this lichen appear black to the naked eye. Apothecia circular or angular, 0.3-1.0 $\mathrm{mm}$. in diameter, immersed; disk black, flat to convex; proper margin concolorous with the disk, black, thin, flexuous, becoming indistinct; hypothecium hyaline or brownish red; spores hyaline, spherical or ellipsoid, nonseptate, 3.3-4.0 × 2.0-3.0 $\mu$.

On rock: Whatcom Co.: Ruth Mountain, $6800 \mathrm{ft} .$, 1928. L. Sunquist 1923 (W).

This alpine lichen can be identified easily by the very black hypothallus.

4. Biatorella fossarum (Duf.) Th. Fr. Lich. Scand. 1:397. 1871-74. Lecidea fossarum Duf. in Fries Lichenogr. Eur. Reform. 264. 1831.

Biatora fossarum (Duf.) Mont.

Thallus pale greenish gray, thin, scurfy, scattered or compacted into a crust. Apothecia circular, 0.5-1.0 mm. in diameter, adnate; disk pale yellow, tawny (R), reddish brown, convex becoming hemispherical; proper margin disappearing early; hypothecium brownish yellow; spores hyaline, oblong, nonseptate, $5.0-11.0 \times 3.0 \mu$. 
On soil: Washington Territory, 1883, Suksdorf 225 (T). Fink (1935) reports this species from Washington.

5. Biatorella moriformis (Ach.) Th. Fr. Lich. Scand. $1: 401.1871-74$. Arthonia moriformis Ach. Syn. Lich. 5. 1814.

Biatora moriformis (Ach.) Tuck.

Thallus grayish white, greenish gray, or brownish gray, indistinct, thin, effuse, smooth, or sometimes granulose and scurfy, often disappearing entirely. Apothecia circular, 1-3 mm. in diameter, adnate; disk dark brown or black, flat to slightly convex ; proper margin soon disappearing ; hypothecium hyaline; spores hyaline, spherical, nonseptate, $1.0-3.5 \mu$ in diameter.

On bark of trees or decaying wood: Washington Territory, 1882, Suksdorf 115 (T). Fink (1935) reports this species from Washington.

\section{Acarospora Mass. Ricerch. Auton. Lich. 27. 1852.}

Thallus uniformly crustose, squamulose, or areolate, often marginally lobed, dorsi-ventral, with a distinct upper cortex, algal and medullary layers, sometimes the medulla is lacking, and with or without a more or less distinct but thin lower cortex, attached to the substratum by hyphal rhizoids. Apothecia one to several in each areole, circular or irregular in shape, usually immersed, rarely sessile; thalline margin present; hypothecium hyaline, rarely dusky; paraphyses unbranched, coherent or distinct, occasionally slimy; asci many-spored; spores very numerous, usually more than 100 , very small, hyaline, nonseptate.

A. Thallus some shade of yellow.

B. On soil, usually sulphur color, indeterminate..............2. A. Schleicheri

B. On rock, bright lemon color, determinate. 3.A. flava

A. Thallus not some shade of yellow.

C. Lower surface of squamules pale brown, thalline margin broad. 4. A. Washingtonensis

C. Lower surface of squamules very dark brown or black, thalline margin thin. 1. A. fuscata

1. Acarospora fuscata (Nyl.) Arn. Verhandl. Zool.-Bot. Gesellsch. Wien. $20: 528.1870$.

Lichen fuscatus Schrad. Spicil. Fl. German. 83. 1794.

Lecanora fuscata (Schrad.) Röhl.

Thallus light or dark brown, reddish or yellowish brown, indeterminate, consisting of squamules, often clustered and areolate, or scattered and lobate, smooth, opaque, or shiny, edges usually free from the substratum, and raised; below dark brown or black. Apothecia numerous, usually solitary, but sometimes several in each areole or squamule, minute, 0.2-0.5 mm. in diameter, immersed; disk darker than the thallus, often almost black, punctiform, then concave to flat, smooth or slightly rough; thalline margin thin, disappearing; hypothecium hyaline; paraphyses coherent; spores hyaline, oblong to subspherical, nonseptate, $4.0-5.0 \times 1.0-2.0 \mu$. 
On rock: Chelan Co.: Rainbow Trail, 3500 ft., 1931, 1266. Okanogan Co. : Tonasket, 1200 ft., 1388.

2. Acarospora Schleicheri (Ach.) Mass. Ricerch, Auton. Lich. 27. 1852.

Urceolaria Schleicheri Ach. Lichenogr. Univers. 332. 1810.

Lecanora Schleicheri (Ach.) Nyl.

Thallus pale sulphur color or yellowish-white, tartareous, indeterminate, consisting of squamules, rather thick, rough, flat to convex, often crowded and angular, margin somewhat lobate or crenate; below reddish brown. Apothecia usually solitary but occasionally 3-4 in each squamule, 0.4-1.0 $\mathrm{mm}$. in diameter, immersed; disk reddish brown or black, flat, slightly roughened; thalline margin concolorous with the thallus, entire or crenate; hypothecium hyaline; paraphyses subdistinct; spores hyaline, almost spherical, nonseptate, 3.0-4.0 x 1.5-2.0 $\mu$.

On soil: Ferry Co.: Republic, 1912, Foster 2367 (F). Fink (1935) reports this species from the state.

When moist this is a very conspicuous lichen because of the bright yellow squamules and the reddish brown disks of the apothecia.

3. Acarospora flava (Bell.) Trevis. Revista Periodic. Lavori I.R. Accad. Padova, 262. 1851-52.

Lichen flavus Bell. app. F1. Pedemont. 261. 1792.

Lecanora chloroplana Ach.

Thallus intensely bright lemon yellow $(R)$, sometimes with a tinge of green, determinate, consisting of squamules, areolate or of elongated squamules, folded, smooth, or warty, convex, marginally lobate, lower cortex lacking. Apothecia crowded towards the center of the lichen mat, 1-several in each squamule, immersed or adnate; disk yellow or reddish brown, flat or convex; thalline margin concolorous with the thallus, entire, or flexuous; hypothecium hyaline; paraphyses distinct; spores hyaline, oblong or oval, nonseptate, $2.0-4.0 \times 1.0-2.0 \mu$.

On rock: Yakima Co.: Naches, gulch near Gleason's Ranch, 1350 ft., 1931, 909; 1940, 3173. Chelan Co.: butte near Chelan, 2500 ft., 1931, 1358. Ferry Co.: Republic, 1912, Foster 2320 (F). Whitman Co.: Pullman, 1900, R. K. Beattie 45 (F).

A showy and beautiful rock lichen, rather common east of the Cascades.

4. Acarospora washingtonensis H. Magn. Ann. Crypt. Exot. 6:46. 1933.

Thallus various shades of brown, indeterminate, consisting of squamules, scattered or somewhat clustered, verrucose or smooth, irregularly convex; below pale brown. Apothecia solitary or occasionally 2-3 in each squamule, $0.3-0.6 \mathrm{~mm}$. in diameter, immersed; disk dark brown, flat to convex; thalline margin concolorous with the thallus, well developed, broad; hypothecium hyaline; paraphyses distinct; spores hyaline, broadly ellipsoid or subglobose, nonseptate, $3.5-5.5 \times 3.5-4.0 \mu$. 
On rock: Yakima Co.: Naches, gulch near Gleason's Ranch, 1350 ft., 1931, 908a, type specimen (W). Chelan Co.: Beaver Summit, 2800 ft.. 1940, 3353.

\section{PERTUSARIACEAE}

Thallus uniformly crustose, normally superficial, seldom developed within the substratum, upper cortex present or absent, algal layer and medulla well developed, but lower cortex lacking, attached to the substratum by hyphal rhizoids. Apothecia circular, 1-several in each apothecial wart, immersed; proper margin lacking, thalline margin present; asci 1-8-spored; spores hyaline or brown, usually large and thick walled, nonseptate or 1-septate.

Alga Protococcus.

45. Pertusaria DC. in Lam. \& DC. F1. Franc. 3rd ed., 2:319. 1805.

Thallus crustose, thin or thick, usually superficial, seldom partly within the substratum, with a poorly developed plectenchymatous upper cortex, well developed algal layer and medulla, and lower cortex lacking. Apothecia 1 -several in each wart, circular, to globular, immersed; thalline margin breaking up into a soredial mass, or firm and well developed. Apothecia opening by a small ostiole or forming a typical disk; hypothecium hyaline; paraphyses branched, interwoven to form a net, asci 1-8-spored; spores usually hyaline, rarely brown, large or very large, thick walled, nonseptate.

A. Thallus bearing many heaps of white soredia.

B. Thallus sterile, tastes bitter, like quinine.

1. P. amara

B. Thallus not always sterile, has no quinine taste........4. P. multipuncta

A. Thallus not bearing many heaps of white soredia, if present, not abundant.

C. Thalline margin thick, regular, rarely disappearing, spores large, often more than $200 \mu$ long. 6. P. velata

C. Thalline margin thin, irregular, sometimes disappearing.

D. Disk prominent, well developed.

E. Disk black, asci usually 2-spored.

5. P. pustulata

E. Disk not black, asci 8-spored.

2. P. ambigens

D. Disk absent, indistinct black ostioles present.

3. P. leioplaca

1. Pertusaria amara (Ach.) Nyl. Bull. Soc. Linn. Normandie ser. 2. $6: 288.1872$.

Variolaria amara Ach. Kgl. Vetensk. Akad. Nya Handl. 163. 1809.

Thallus light yellowish olive $(R)$ or grayish white, becoming widely spread, rough and cracked, somewhat powdery, bearing numerous heaps of white soredia, which are often confluent, margins zonate. Apothecial warts adnate, flat, sterile; thalline margin irregular, these warts develop into the powdery soredial heaps, that almost cover the central part of the thallus. These apothecial warts taste bitter like quinine; yellow then reddish with $\mathrm{KOH}$, violet with $\mathrm{KOH}+\mathrm{CaOCl}_{2}$. 
On bark: San Juan Co.: San Juan Island, Friday Harbor, 1928, 2025. King Co.: Edgewood, $300 \mathrm{ft}, 1940,3087$. Thurston Co.: Grand Mound, 1940, 2942; Gate, 1940, 2969. Clallam Co. : Sequim, 1915, J. M. Grant 419 (F). Island Co.: Whidbey Island, Langley, 1920, J. M. Grant 3043.

The quinine taste and the color reactions of this lichen make it an easy one to identify.

2. Pertusaria ambigens (Nyl.) Tuck. Proc. Am. Acad. Arts \& Sci. 12:176. 1877.

Lecanora ambigens Nyl. Act. Soc. Sci. Fenn. 7 :449. 1863.

Thallus pale greenish gray or darker gray, smooth becoming rough and warty. Apothecia usually only one in each wart, $0.2-1.0 \mathrm{~mm}$. in diameter, sessile or somewhat elevated; disk pale yellow, flesh color (R), or green, sometimes gray pruinose, concave to flat; thalline margin concolorous with the thallus, irregularly crenate, often torn and separated to form several layers; asci 8-spored; spores hyaline, ellipsoid, nonseptate, 16.5-21.4 x 9.9-12.0 $\mu$, with rather a wide epispore. Thallus yellow with $\mathrm{KOH}$.

On bark: Clallam Co.: Elwha River Road, 250 ft., 1940, 2897a. San Juan Co.: San Juan Islands, 1906, E.T.ES.A.Harper 237 (F). King Co.: Summit, 1907, Foster 6681/2 (F).

3. Pertusaria leioplaca (Ach.) DC. in Lam. \& DC. Fl. Franc. 3rd ed., $6: 173.1815$.

Porina leioplaca Ach. Kgl. Vetensk-Akad. Nya Handl. 159. 1809.

Thallus white, pale greenish gray, or light buff ( $R$ ), thin, smooth, or slightly cracked, rough and scaly, sometimes partly developed within the substratum, apothecial warts rather numerous, scattered, smooth and prominent, with several apothecia in each wart. Apothecia 0.2-0.4 mm. in diameter, depressed at the center; disk lacking, ostioles black, punctiform, often indistinct; thalline margin irregular, at times disappearing; asci 4-6-8-spored; spores hyaline to pale brown, ellipsoid, nonseptate, 44.0$184.0 \times 22.0-56.0 \mu$, a great variation in spore sizes. Thallus faintly yellow with $\mathrm{KOH}$.

On bark or rock: Clallam Co.: Port Angeles, 1914, Foster 2648 (F). Island Co. : Whidbey Island, Langley, 1923, J. M. Grant 216e (F). Thurston Co.: Gate, 1913, Foster 2012 (W).

4. Pertusaria multipuncta (Turn.) Nyl. Lich. Scand. 179. 1861.

Variolaria multipuncta Turn. Trans. Linn. Soc. London, 9:137. 1808.

Thallus pale olive-buff (R), deep olive-buff $(R)$, warm buff $(R)$, or gray, rough, warty and wrinkled, indistinctly zonate at the margin. Apothecia 1-several in each wart, 0.2-0.8 mm. in diameter, immersed. Apothecia closed at first, then with black ostioles, and finally becoming white soredial heaps; disk, if present, black, white, or pale yellow; thalline margin ir- 
regular; asci 1-2-spored; spores hyaline, oblong-ellipsoid, nonseptate, 82.0-155.1 $\times 28.0-68.0 \mu$. Thallus yellow then bright red with $\mathrm{KOH}$.

On bark or rock: Spokane Co.: Mt. Spokane, 5808 ft., 1931, 1486. Wahkiakum Co.: Cathlamet, 1940, 2861. Clallam Co.: Olympic Hot Springs, 1935, A. H. Smith 2125 (F). San Juan Co.: San Juan Island, Roche Harbor, 1905, Frye (F); Orcas Island, Turtle Back Mountain, 1906, Fink 453 (W). Island Co.: Whidbey Island, J. M. Grant 3153 (F).

The definite zonate margin and lack of the quinine taste help to distinguish this Pertusaria.

5. Pertusaria pustulata (Ach.) Duby, Bot. Gallic. $2: 673.1830$.

Porina pustulata Ach. Lichengr. Univers. 309. 1810.

Thallus white, olive-buff ( $R)$, dark olive-buff $(R)$, or cream-buff $(R)$, thin, smooth or rough, cracked and slightly warty. Apothecia 1-several in each wart, hemispherical or difform, 0.2-0.5 mm. in diameter, at first immersed, then becoming globular with prominent ostioles, finally ostioles unite to form black, flat disks; thalline margin concolorous with the thallus, quite thick, irregular; asci 2-spored; spores hyaline, ellipsoid, nonseptate, $70.5-85.0 \times 30.0-40.0 \mu$. Thallus yellow with $\mathrm{KOH}$.

On bark: Washington Territory, Suksdorf 271 (T). Herre (1917) reports this species, with some question, from Whatcom Co.

6. Pertusaria velata (Turn.) Nyl. Lich. Scand. 179. 1861.

Parmelia velata Turn. Trans. Linn. Soc. London, 9:143. 1808.

Thallus white or pale greenish white, thin, smooth or cracked, wrinkled and folded, zonate at the margin. Apothecia 1-several in each wart, warts crowded, apothecia $0.3-1.0 \mathrm{~mm}$. in diameter, immersed; disk pale yellow or flesh-color ( $R$ ), densely white pruinose, concave to flat; thalline margin concolorous with the thallus, regular, thick, very rarely disappearing; asci 1 -spored, or rarely 2 -spored; spores hyaline, oblong-ellipsoid, nonseptate, 184.0-230.0 $\times 42.0-60.0 \mu$. Thallus shows no color change with $\mathrm{KOH}$.

On bark or rock: San Juan Co.: San Juan Island, Friday Harbor, trail to Heaven, 1940, 3246. Kitsap Co.: Hidden Ranch near Bremerton, 213 ft., 1940, 3289. Clallam Co.: Ozette Lake, A. H. Smith 13433 (F). Grays Harbor Co.: Westport, 1908, Foster $670 b$ (F).

The absence of white soredial heaps and the lack of any color change with $\mathrm{KOH}$ characterize this Pertusaria, and separate it definitely from $P$. multipuncta, with which it is sometimes confused.

\section{LECANORACEAE}

Thallus uniformly crustose, sometimes marginally lobed, subfoliose, squamulose or rarely dwarf fruticose, often differentiated into cortex, algal and medullary layers, but no differentiation in some species; attached to the substratum by hyphal rhizoids or an umbilicus. Apothecia 
circular, scattered, immersed, adnate or sessile, or rarely stipitate, proper margin usually absent, thalline margin well developed, asci 4-32-spored; spores hyaline, rarely brownish, nonseptate, 2-many-septate, or muriform, thin walled.

Alga Protococcus or Protococcus and Gloeocapsa.

A. Spores nonseptate.

B. Spores large always more than $30 \mu$ long; paraphyses branched, coherent. 47. Ochrolechia

B. Spores smaller; paraphyses unbranched, distinct or coherent.

C. Thallus usually egg-yellow or orange; disk always some shade of yellow. No color change with $\mathrm{KOH}$.....................51. Candelariella

C. Thallus very seldom egg-yellow or orange; disk not often yellow; thallus, if yellow, turns bright yellow, red, or purple with $\mathrm{KOH}$. 46. Lecanora

A. Spores septate.

D. Spores 1-3-septate.

E. Spores usually 1-septate.

F. Thallus usually egg-yellow or orange; disk always some shade of yellow. No color change with KOH................51. Candelariella

F. Thallus never egg-yellow or orange, sometimes pale yellow; disk never yellow; no color change with $\mathrm{KOH}$................49. Lecania

E. Spores usually more than 1-septate, paraphyses unbranched, distinct. 48. Icmadophila

D. Spores 3-many-septate, disk purplish blue with $\mathrm{KOH}$.

50. Haematomma

46. Lecanora Ach. Lichenogr. Univers. 77. 1810.

Thallus crustose, marginally lobed, squamulose, rarely subfoliose or very rarely fruticose; crustose species have no differentiation into layers, or only poorly developed upper cortex, algal layer, and medulla; foliose forms have a well-developed, gelatinized, pseudocellular upper cortex, an algal layer and a medulla, but a poorly developed lower cortex, attached to the substratum by hyphal rhizoids or an umbilicus. Apothecia circular, immersed, adnate or sessile, with a poorly developed proper margin, but a distinct thalline margin ; hypothecium hyaline or colored; paraphyses distinct or coherent and unbranched; asci normally S-spored, rarely 4-16-32spored; hyaline; nonseptate, thin walled without a halo. A large and difficult genus.

Alga Protococcus or Protococcus and Gloeocapsa.

A. Always on rock (Placodium).

B. Bearing cephalodia and soredia. 25. L. gelida

B. Bearing neither cephalodia nor soredia.

C. Thallus squamulose, not marginally lobed.

D. Attached to the substratum by an umbilicus, thallus small, consisting of closely united squamules.........................22. L. peltata

D. Not attached to the substratum by a single, definite umbilicus. 
E. Disk greenish gray or black.

24. L. rubina var. melanophthalma

E. Disk yellow, fawn color, or reddish brown............23. L. rubina

C. Thallus subfoliose, marginally lobed.

F. Loosely attached to the substratum

F. Closely attached to the substratum.

20. L. melanaspis

21. L. muralis

A. On rock, bark, old wood, or moss; crustose, neither squamulose, subfoliose, nor marginally lobed.

B. Apothecia adnate or sessile, disk flat to convex (Eulecanora).

C. On bark, old wood, or moss.

D. Disk pruinose.

E. Thallus never areolate, yellow then brick red with $\mathrm{KOH}$.

E. Thallus often areolate, yellow with $\mathrm{KOH}$.

13. L. pallida

D. Disk not pruinose.

F. Apothecia reddish brown or black.

G. Hymenium and hypothecium brown or blackish.... 8. L. atra

G. Hymenium and hypothecium not brown or blackish.

$\mathrm{H}$. Apothecium not crowded, thalline margin prominent, always on bark.

17. L. subfusca var. argentata

$\mathrm{H}$. Apothecia crowded, thalline margin not always prominent, not always on bark.

16. L. subfusca

F. Apothecia pale yellow, buff, reddish brown, or rarely black.

I. Thallus white or greenish gray, no color change with $\mathrm{KOH}$. 10. L. Hageni

I. Thallus white or pale yellow.

J. Only on old wood, near the ocean, thallus yellow then orange with $\mathrm{KOH}$.

18. L. symmicta

J. On bark, old wood, or moss, thallus yellow with $\mathrm{KOH}$.

19. L. varia

C. On rock.

$\mathrm{K}$. Thallus absent.

14. L. polytropa

$\mathrm{K}$. Thallus present.

L. Disk pruinose.

15. L. rupicola

L. Disk not pruinose.

M. Apothecia reddish or black.

N. Alga Gloeocapsa and Protococcus...............9. L. granatina N. Alga Protococcus.

O. Hymenium and hypothecium black.

8. L. atra

O. Hymenium and hypothecium not black.

P. Apothecia crowded, usually irregular in shape;

thallus sparse.

16. L. subfusca

P. Apothecia not crowded, usually circular; thallus abundant, areoles often centrally stalked.

11. L. oregana

M. Apothecia pale yellow, buff, or very rarely black.

$\mathrm{Q}$. Thallus white or greenish gray, no color change with 
$\mathrm{KOH}$. 10. L. Hageni

Q. Thallus white or pale yellow, yellow with $\mathrm{KOH}$.

19. L. varia

B. Apothecia immersed or adnate; disk concave to flat (Aspicilia)

R. Disk pruinose.

S. On soil, moss, or bark, spores usually more than $30 \mu$ long.

7. L. verrucosa

S. On rock, spores less than $30 \mu$ long.

2. L. calcarea

R. Disk naked.

$\mathrm{T}$. Thallus yellow then brick-red with $\mathrm{KOH}$.

U. Medulla blue with IKI.

1. L. alpina

U. Medulla not blue with IKI.

3. L. cinerea

$\mathrm{T}$. Thallus shows no color change with $\mathrm{KOH}$.

V. Thallus brick red or pale brick red.

5. L. lacustris

V. Thallus neither brick red nor pale brick red.

W. Thallus rough and warty, crumbling, apothecia finally becoming adnate.

4. L. gibbosa

W. Thallus smooth, not warty, apothecia remaining immersed.

6. L. laevata

1. Lecanora alpina Somrft. Suppl. Fl. Lapp. 91. 1826.

Thallus gray or bluish gray, often pale buff at the margins, crustose, consisting of minute, round or irregular warty areoles, separated by rather wide fissures on a black hypothallus; yellow with $\mathrm{KOH}$, then a permanent reddish color, no color change with $\mathrm{CaOCl}_{2}$, medulla blue with IKI. Apothecia circular, numerous, $1-3$ in an areole, $0.2-1.0 \mathrm{~mm}$. in diameter, immersed to finally emergent, and even elevated; disk reddish black or black, flat; thalline margin concolorous with the thallus, thin, entire, rarely disappearing; asci 8-spored; spores hyaline, ovoid, nonseptate, 8.0-13.2 x 7.0-9.0 $\mu$.

On rock: Pierce Co.: Mount Rainier, Yakima Park, 6300 ft., 1931, 1235. Clallam Co.: Olympic Mountains, Storm King Mountain, $3500 \mathrm{ft}$, A. H. Smith 2230 (W). Herre (1917) reports this lichen rare from Mt. Baker, above the snow line.

2. Lecanora calcarea (L.) Somrft. Suppl. F1. Lapp. 102. 1826.

Lichen calcareus Linn. Sp. P1. 1140. 1753.

Thallus white, greenish gray, or bluish, crustose, rough and crumbly, consisting of contiguous, angular areoles, usually effigurate at the circumference; no color change with $\mathrm{KOH}, \mathrm{CaOCl}_{2}$, or IKI. Apothecia circular, 0.2-0.8 $\mathrm{mm}$. in diameter, immersed to rarely adnate; disk brown or black, more or less pruinose, flat; thalline margin concolorous with the thallus, thin; asci usually 6 -spored; spores hyaline, ellipsoid to globose, nonseptate, $16.0-26.0 \times 9.0-20.0 \mu$. 
On rock: San Juan Co. : San Juan Island, trail to Heaven, 1940, 3248; Argyle, 1940, 3214. Okanogan Co.: butte near Twisp, 1700 ft., 1931, 1464. Herre (1917) reports it from Mt. Baker at $6000 \mathrm{ft}$.

3. Lecanora cinerea (L.) Röhl. Deutschl. Fl. 3:2 Abt. 90. 1813.

Lichen cinereus Linn. Mant. $1: 132.1767$.

Thallus ashy-white, gray, brownish gray, or almost black, crustose, consisting of rough uniform areoles separated by irregular fissures, or of separate distinct areoles; black hypothallus not always evident; yellow with $\mathrm{KOH}$, then a permanent reddish color, no color change with either $\mathrm{CaOCl}_{2}$ or IKI. Apothecia circular to somewhat irregular in shape, numerous, 1 -several in an areole, $0.2-1.0 \mathrm{~mm}$. in diameter, immersed; disk black, concave to flat; thalline margin concolorous with the thallus or sometimes black, thin, persistent; asci usually 8-spored, sometimes 6-spored; spores hyaline, ellipsoid or subglobose, nonseptate, 18.0-22.0 x 13.2-14.0 $\mu$.

On rock: Okanogan Co.: Tonasket, $1200 \mathrm{ft} ., 1931,1384$. Ferry Co.: north of Republic, 2650 ft., 1931, 1403; Mt. Gibraltar, 3100 ft., 1940. Whitman Co.: Pullman, 2550 ft., 1931, 1584a. Garfield Co.: 1860 ft., 1931, 1569. Clallam Co.: Lake Crescent, Storm King Mountain, 1935, A. H. Smith 2283 (F). Rainier National Park, Glacier Basin, 1925, J. M. Grant (F).

4. Lecanora gibbosa (Ach.) Nyl. Mem. Soc. Imp. Sc. Nat. Cherbourg, $5: 113.1857$.

Lichen gibbosus Ach. Lichenogr. Suec. Prodro. 30. 1798.

Lecanora cinerea var. gibbosa (Ach.) Nyl.

Thallus gray, greenish brown, or brown, crustose, determinate, consisting of rather thick, smooth areoles, becoming tumid, irregular, rough and crumbling, on a black hypothallus; no color change with $\mathrm{KOH}$, $\mathrm{CaOCl}_{2}$, or IKI. Apothecia circular to somewhat irregular in shape, 1, 2, or 3 in an areole, $0.4-1.0 \mathrm{~mm}$. in diameter, immersed, finally adnate; disk black, concave to flat; thalline margin concolorous with the thallus, often blackening, persistent, thin, becoming slightly crenulate; asci 4-8-spored, ellipsoid or subglobose, with a distinct epispore, nonseptate, 20.0-23.1 x 8.5-9.0 $\mu$.

On rock: Chelan Co.: White Rock Camp, 2200 ft., 1931, 1040; Dirty Face Mountain, 3000 ft., 1931, 1369. Yakima Co.: gulch near Naches, 1350 ft., 1931, 1166. Okanogan Co.: Twisp State Trail, 5000 ft., 1931, 1303; Olympic Mountains, Hurricane Ridge, 5500 ft., A. H. Smith 2876 (M). Ferry Co.: Republic, 1912, Foster 2319 (F). Washington Territory, Suksdorf (S).

5. Lecanora lacustris (With.) Nyl. Lich. Scand. 155. 1861.

Lichen lacustris With. A Bot. Arr. Brit. P1. 3rd ed., 4 :21. 1796.

Thallus brick color or pale brick color, crustose, thin, smooth, rimoseareolate; no color change with $\mathrm{KOH}, \mathrm{CaOCl}_{2}$, or IKI. Apothecia circular, 
0.2-0.6 mm. in diameter, immersed, often urceolate; disk reddish brown or black, concave; proper margin rather thick, concolorous with the thallus, or brownish black, sometimes difficult to distinguish from the thallus, becoming flexuous; asci 8 -spored; spores hyaline, ellipsoid, nonseptate, $11.0-18.0 \times 5.0-8.0 \mu$.

On rock, often inundated: Pierce Co.: Mt. Rainier, Berkeley Park, 6000 ft., 1940, 3040. Yakima Co.: Lodge Pole Pine Camp, 3500 ft., 1940, 3305. Washington Territory, Suksdorf 273 (S).

The brick red thallus makes this a very easy Lecanora to identify.

6. Lecanora laevata (Ach.) Nyl. in Not. ur Sällsk. Faun. et Fl. Fenn. Forhandl. 8:137. 1866.

Sagedia laevata Ach. Kgl. Vet.-Akad. Nya Handl. 164. 1809.

Lecanora cinerea var. laevata (Ach.) Tuck.

Thallus dirty gray, greenish gray, or pale brown, crustose, thin, smooth or slightly rough, cracked, sometimes almost angular, areolate, at times showing a black hypothallus; no color change with $\mathrm{KOH}, \mathrm{CaOCl}_{2}$, or IKI. Apothecia circular, 0.2-0.5 mm. in diameter, immersed; disk black, concave; thalline margin concolorous with the thallus, often hard to distinguish from the thallus, thin, then swollen; asci usually 8 -spored; spores hyaline, ellipsoid, nonseptate, $15.0-31.5 \times 9.0-15.0 \mu$.

On rock: Clallam Co.: Olympic Hot Springs, A. H. Smith 2147 (M). Herre (1943) reports this species from Mt. Baker, $6000 \mathrm{ft}$., Whatcom Co. Fink (1935) reports it from Washington.

7. Lecanora verrucosa Ach. Lich. Univ. 354. 1810.

Urceolaria verrucosa Ach. Lich. Univ. 339. 1810.

Parmelia verrucosa (Ach.) Sprgl.

Thallus white, gray, or greenish gray, crustose, rather thick, warty (verrucose), tartareous (powdery); no color changes with $\mathrm{KOH}, \mathrm{CaOCl}_{2}$, or IKI. Apothecia circular, 0.5-1.0 mm. in diameter, immersed, finally subsessile; disk black, deeply concave to flat, sometimes grayish pruinose, often almost closed; proper margin thin, hidden more or less by the thalline margin, which is concolorous with the thallus, at first rounded and inflexed, becoming flexuous; asci 8-spored; spores hyaline, broadly ellipsoid, nonseptate, $30.0-53.0 \times 16.0-37.0 \mu$.

On moss and soil: Klickitat Co.: Goldendale, 1909, Foster 1156 (F). Washington Territory, Brandegee 581 (F).

This lichen is characterized by the habitat and the large sized spores.

8. Lecanora atra (Huds.) Ach. Lichenogr. Univers. 344. 1810.

Lichen ater Huds. Fl. Ang. 1:445. 1762.

Thallus grayish white, Isabella color ( $R$ ), or deep olive buff ( $R$ ), crustose, moderately thick, composed of granules, becoming warty, and often areolate; yellow with $\mathrm{KOH}$, no color change with $\mathrm{CaOCl}_{2}$ or IKI. 
Apothecia circular, $0.5-2.0 \mathrm{~mm}$. in diameter, somewhat immersed, adnate to subsessile; disk black, sometimes shiny, finally tumid, brown or black within, flat to convex, paraphyses violet to black, or dull bluish violet; thalline margin concolorous with the thallus, rarely becoming black, entire, rarely crenate or flexuous; asci 8-spored; spores hyaline, ellipsoid, nonseptate, $10.0-15.0 \times 5.0-8.5 \mu$.

On rock or bark: San Juan Co.: San Juan Island, Argyle, 1940, 3215.

The violaceous paraphyses help to distinguish this Lecanora from others with a black disk.

9. Lecanora granatina Somrft. Suppl. Fl. Lapp. 90. 1826.

Pannaria granatina (Somrft.) Th. Fr.

Thallus reddish brown or cinnamon-drab ( $R$ ), crustose, consisting of minute, round or irregular shaped granules, rather thick, rough and warty, whitish within; below blackening, no color change with $\mathrm{KOH}, \mathrm{CaOCl}_{2}$, or IKI; attached at one point to the substratum. Apothecia circular, 0.2-0.8 $\mathrm{mm}$. in diameter, adnate; disk chestnut or reddish brown, naked, shiny, flat to convex; thalline margin concolorous with the thallus, thin, subcrenulate; asci 8-spored; spores hyaline, oblong, nonseptate, 9.9-14.0 x 4.0-6.6 $\mu$.

Alga Protococcus and Gloeocapsa.

On rock: Kitsap Co.: Hidden Ranch near Bremerton, 213 ft., 1931, 1750. Yakima Co.: Little Naches, 2557 ft., 1931, 1170.

10. Lecanora Hageni Ach. Lichenogr. Univers. 367. 1810.

Lichen Hageni Ach. Lichenogr. Suec. Prodro. 57. 1798.

Thallus greenish gray or dirty white, crustose, thin, smooth, becoming rough and warty, scattered and sometimes disappearing altogether; no color change with $\mathrm{KOH}, \mathrm{CaOCl}_{2}$, or IKI. Apothecia circular, 0.5-1.0 mm. in diameter, adnate to sessile: disk pale reddish brown, or black, naked or gray-pruinose, flat to convex; thalline margin white or concolorous with the thallus; asci 8-spored; spores hyaline, ellipsoid, nonseptate, 8.0-14.0 $\mathrm{x}$ 4.9-6.6 $\mu$.

On bark, old wood, or rock: San Juan Co.: San Juan Island, Roche Harbor, 1931, 2034. Yakima Co.: Bumping Lake, 3400 ft., 1931, 939. Chelan Co.: White Rock Camp, 2200 ft., 1931, 1042. Kittitas Co.: Cle Elum, 2200 ft., 1931, 865. Spokane Co. : Mt. Spokane, 5808 ft., 1931, 1487. Clallam Co.: Dungeness, 1913, Foster 2531 (F). Whitman Co.: Pullman, 1900, R. K. Beattie 29 (F).

A rather inconspicuous little lichen, but widely distributed in the state.

11. Lecanora oregana Tuck. Syn. N. Am. Lich. 1:193. 1882.

Thallus greenish yellow or deep olive buff, crustose or areolate, consisting of thick, coarse, crowded, often heaped, warty areoles, yellow or 
orange with $\mathrm{KOH}$, no color change with $\mathrm{CaOCl}_{2}$; sometimes attached to substratum by a central stalk. Apothecia circular or irregular in shape, rather large and conspicuous, $1.0-3.5 \mathrm{~mm}$. in diameter, subsessile to sessile; disk chestnut brown or reddish brown, naked, flat to convex; thalline margin concolorous with the thallus, incurved, flexuous, crenulate, rarely disappearing; asci 8-spored; spores hyaline, broadly ellipsoid with rounded ends, nonseptate, $10.5-14.0 \times 6.6-8.0 \mu$.

On rock: Ferry Co. : North of Republic, 2650 ft., 1931, 1407. Hillside near Sanpoil, 2360 ft., 1940, 3143.

\section{Lecanora pacifica Tuck. Syn. N. Am. Lich. 191. 1882.}

Thallus dirty white or gray, crustose, thin and smooth, becoming rough and warty, sometimes areolate; yellow with $\mathrm{KOH}$ but no color change with $\mathrm{CaOCl}_{2}$. Apothecia circular, 0.2-1.0 mm. in diameter, adnate to subsessile; disk pale yellow, tawny (R), or brownish black, usually thin, flat to convex, greenish yellow or white-pruinose; thalline margin thin, white, persistent, crenulate, often flexuous; asci 8-spored; spores hyaline, ellipsoid, nonseptate, $10.7-16.0 \times 6.6-10.0 \mu$.

On bark: Pacific Co.: Loomis Lake, 1931, 749. Clallam Co.: Lake Sutherland, 590 ft., 1931, 1032. Kitsap Co.: Hidden Ranch near Bremerton, $213 \mathrm{ft}$., 1931, 1764. King Co. : Edgewood, $300 \mathrm{ft} ., 1931$, 1825. Kittitas Co.: Easton, 2168 ft., 1931, 833. Yakima Co.: Dead Horse Hill, $4500 \mathrm{ft}$., 1931, 985. Klickitat Co.: Bickleton, 3200 ft., 1931, 1640. Spokane Co.: Spokane, 1910, Bonser 41 (W).

An abundant bark lichen but one is not always too sure of its identity.

13. Lecanora pallida (Schreb.) Rabh. Deutschl. Krypt. Fl. 2:34. 1845.

Lichen pallidus Schreb. Spicil. Fl. Lips., 133. 1771.

Thallus white, pale yellow, or deep olive (R), crustose, thin or thicker, smooth or rough and warty; yellow then red with $\mathrm{KOH}$, no color change with $\mathrm{CaOCl}_{2}$. Apothecia circular, 0.5-3.0 mm. in diameter, adnate to sessile; disk buff or pale reddish brown, white-pruinose, tumid, flat to convex; thalline margin concolorous with the thallus, at first thick, becoming crenulate and flexuous, sometimes disappearing; asci 8-spored; spores hyaline, ellipsoid, nonseptate, 9.0-18.0 x 6.0-11.0 $\mu$.

On bark: No doubt collected by the writer, but so difficult to distinguish from Lecanora pacifica Tuck. that I am not reporting any definite collections. Thurston Co.: Gate, 1912, Foster 1989 (F). Olympic Mountains, Elwha Valley, $1800 \mathrm{ft}$., 1907, Frye 52 (F). Washington Territory, Cascade Mountains, 1883, Brandegee 57 (S).

Lecanora pacifica Tuck. and Lecanora pallida (Schreb.) Rabh. are very similar; L. pallida has somewhat longer spores, it grows on smooth bark, and the thallus never becomes areolate. Yellow then red with $\mathrm{KOH}$ should separate $L$. pallida from $L$. pacifica, but I do not have too much confidence in chemical tests, unless combined with morphological characters. 
14. Lecanora polytropa (Ehrh.) Rabh. Deutschl. Krypt. Fl. 2:37. 1845.

Verrucaria polytropa Ehrh. Hoffm. Deutschl. Fl. 196. 1796.

Lecanora varia var. polytropa (Ehrh.) Dietrich.

Thallus white, pale green, or yellow, crustose, granulate, or areolate, thin and scattered, often disappearing entirely or rather thick and rough; yellowish with $\mathrm{KOH}$, no color change with $\mathrm{CaOCl}_{2}$. Apothecia circular, 0.2-1.0 $\mathrm{mm}$. in diameter, adnate to subsessile; disk yellow, pinkish buff $(F)$, fawn color $(R)$, or brown, naked, flat to convex; thalline margin concolorous with the thallus, thin, flexuous and often disappearing; asci 8-spored; spores hyaline, ellipsoid, nonseptate, 9.0-15.0 x 5.0-7.0 $\mu$.

On rock: Olympic Mountains, Steeple Peak, 1935, A. H. Smith 2313 (F). Mt. Rainier National Park, Glacier Basin, 1925, J. M. Grant 418 (F). Herre (1943).

15. Lecanora rupicola (L.) Zahlbr. Cat. Lich. Univ. 5:525. 1928.

Lichen rupicola Linn. Mant. 1:132. 1767.

Lecanora sordida (Pers.) Th. Fr.

Thallus brownish white or gray, crustose, determinate, areolate, often circular, smooth or cracked, the areoles raised, circumference somewhat squamulose; bright yellow with $\mathrm{KOH}$, no color change with $\mathrm{CaOCl}_{2}$. Apothecia circular, $0.5-1.0 \mathrm{~mm}$. in diameter, immersed, finally adnate; disk usually black, at times pale flesh color, densely pruinose; thalline margin concolorous with the thallus, thin, entire, often crenate and flexuous, finally disappearing; asci 8-spored; spores hyaline, ellipsoid, nonseptate, 9.0-17.0 $\times 5.0-8.0 \mu$.

On rock: San Juan Co.: Shaw Island, 1928, 1949. Clallam Co. : Sequim, 1916, J. M. Grant 556, 559 (F). Olympic Mountains, Hurricane Ridge, 6000 ft., A. H. Smith 2396 (M). Klickitat Co.: Goldendale, 1909, Foster 1192 (F). Ferry Co.: Republic, 1913, Foster 2424 (F).

A variable species, but the densely pruinose disk is constant and makes identification easy.

16. Lecanora subfusca (L.) Ach. Lichenogr. Univers. 393. 1810.

Lichen subfuscus Linn. Sp. Pl. 1142. 1753.

Thallus dirty white, gray, greenish gray, or cream buff $(R)$, crustose, usually thin and smooth or rough, at times becoming thicker, then granulose-verrucose or warty-areolate; yellow with $\mathrm{KOH}$, no color change with $\mathrm{CaOCl}_{2}$. Apothecia circular, numerous crowded, becoming irregular in shape, $0.5-2.5 \mathrm{~mm}$. in diameter, adnate to sessile; disk brown, reddish brown, or brownish black, naked (not pruinose), flat to somewhat convex; thalline margin concolorous with the thallus, thin, entire to crenate or flexuous, persistent; asci 8-spored; spores hyaline, ellipsoid, nonseptate, $13.2-20.0 \times 5.6-11.0 \mu$.

On bark or old wood, rarely on rock or moss: Pacific Co.: Loomis Lake, 1931, 773; Chinook, 1931, 452. San Juan Co.: San Juan Island, Pt. 
Caution, 1928, 2033. Pierce Co.: White River Camp, 3000 ft., 1928, 1922. Yakima Co.: Little Naches, 2557 ft., 1171. Olympic Mountains, Pysht River, 1935, A. H. Smith, 1913 (F). Pierce Co.: Ft. Steilacoom, 1912, Foster (F).

A common Lecanora, often found on drift wood on the ocean beach, but widely scattered through the state. Since I can not separate satisfactorily Lecanora allophana (Ach.) Röhl. from Lecanora subfusca (L.) Ach., I recognize but one species, L. subfusca, thus not following Zahlbruckner (1921-1932) here.

17. Lecanora subfusca var. argentata Ach. Lichenogr. Univers. 393. 1810.

Thallus white or pale gray, always thin, smooth at first, finally somewhat cracked. Apothecia smaller and less crowded than the species, usually circular and not so irregular in shape as the species; thalline margin very prominent, spores smaller than in the species, 9.9-14.0 $\times$ 4.6-7.0 $\mu$, otherwise like the species.

On bark only: Grays Harbor Co.: Lake Quinault, 300 ft., 1931, 1208. Pacific Co.: Loomis Lake, 1931, 736. Wahkiakum Co.: Cathlamet, 1940, 2917. King Co.: Edgewood, 300 ft., 1940, 3091. Grays Harbor Co. : Montesano, 1918, J. M. Grant (WTU).

No doubt there are other varieties in the state, but this is the only one that seems clearly defined to me at present.

\section{Lecanora symmicta Ach. Syn. Lich. 340. 1814.}

Lecanora varia var. symmicta Ach. Lichenogr. Univers. 379. 1810.

Thallus white or pale yellow, crustose, thin or rather thick, smooth, or scurfy and sometimes granulose, rarely disappearing; yellow with $\mathrm{KOH}$, then orange, no color change with $\mathrm{CaOCl}_{2}$. Apothecia circular, often crowded then becoming irregular in shape, $0.4-1.0 \mathrm{~mm}$. in diameter, adnate; disk pale yellow, reddish, tawny-olive ( $R$ ), sometimes blackening; thalline margin concolorous with the thallus, thin, finally disappearing; asci 8-spored; spores hyaline, ellipsoid, nonseptate, 9.0-15.0 x 4.0-6.0 $\mu$.

On old wood: Clallam Co. : Dungeness, 1913, Foster 2560 (F).

Found only near the ocean in Washington.

19. Lecanora varia (Ehrh.) Ach. Lichenogr. Univers. 377. 1810.

Lichen varius Ehrh. P1. Crypt. Exs. no. 68. 1785.

Thallus white, pale yellow, or pale green, crustose, bordered somewhat by a black hypothallus, thin, smooth, or warty, areolate or of distinct granules, sometimes scanty; yellow with $\mathrm{KOH}$, no color change with $\mathrm{CaOCl}_{2}$. Apothecia circular, numerous, often crowded, becoming irregular in shape, 0.2-0.8 mm. in diameter, adnate to subsessile; disk fawn color (R), yellow, or warm buff $(R)$, naked, flat to convex; thalline margin concolorous with the thallus, thin, erect, entire or crenulate, finally disappearing; asci 8-spored; spores hyaline, ellipsoid, nonseptate, 9.4-14.9 x 4.0-7.0 $\mu$. 
On bark, wood, more rarely on rocks: King Co.: Snow Lake Trail, 3500 ft., 1931, 1135. San Juan Co. : San Juan Island, Roche Harbor, 1905, Frye (F). San Juan Island, 1906, E. T. and S. A. Harper (F).

20. Lecanora melanaspis Ach. Lichenogr. Univers. 427. 1810.

Parmelia melanaspis Ach. Meth. Lich. 196. 1803.

Thallus whitish, gray, warm buff $(\mathrm{R})$, reddish brown, or brown, subfoliose, thick, becoming turgid, in the center warty-areolate, at the circumference linear, stellate-imbricate lobed; yellow then red almost immediately with $\mathrm{KOH}$, no color change with $\mathrm{CaOCl}_{2}$. Apothecia circular to angular, 0.4-2.0 mm. in diameter, adnate to subsessile; disk reddish black, brownish black, or black, sometimes pruinose, often tumid, flat to convex; thalline margin concolorous with the thallus, becoming irregular, rarely disappearing; asci 8-spored; spores hyaline, ellipsoid or ovoid, nonseptate, 8.6-13.2 × 7.0-9.0 $\mu$.

On rock: Yakima Co.: cliff near Naches Ranger Station, $2400 \mathrm{ft}$., 1940, 3317. Ferry Co.: Mt. Gibraltar, 3100 ft., 1940, 2762. Garfield Co.: Pomeroy, 1860 ft., 1574. Ferry Co. : Republic, 1913, Foster 2395 (F). This specimen determined as L. melanaspis var. stellata Th. Fr. by Zahlbruckner, but this variety is now included in the species. Washington Territory, Brandegee 22 (S).

21. Lecanora muralis (Schreb.) Rabh. Deutschl. Krypt. Fl. 2:42. 1845.

Lichen muralis Schreb. Spicil. Fl. Lips. 130. 1771.

Thallus light greenish-olive (R), pale yellow, or brown, subfoliose, orbicular, tartareous, in the center consisting of scales or areoles, at the circumference of sinuate lobes, flat or plicate with crenate margins; no color change with $\mathrm{KOH}$ or $\mathrm{CaOCl}_{2}$; rather closely attached to the substratum by hyphal rhizoids. Apothecia circular to angular, due to crowding, 0.4-1.0 mm. in diameter, adnate to subsessile; disk yellow, tawny $(\mathrm{R})$, or brown, flat; thalline margin concolorous with the thallus, thin, crenate, flexuous, persistent; asci 8-spored; spores hyaline, ellipsoid, nonseptate, 8.0-16.0 x 4.0-7.0 $\mu$.

On rock: Chelan Co.: Beaver Summit, 2800 ft., 1931, 1336; Zena, 800 ft., 1931, 1107b; Junior Pt. Lookout, 7000 ft., 1940, 3112. Columbia Co.: Dayton, $1700 \mathrm{ft} ., 1940,2727,2735$. Klickitat Co.: Goldendale, 1909, Foster 1166 (F). Spokane Co.: Spokane, 1907, Bonser (F).

The close attachment of the bluish black tinged and lobed margins of the thallus to the substratum is a definite distinguishing characteristic for this species.

\section{Lecanora peltata (Ram.) Steud. Nomenclat. Bot. 237. 1824.}

Lichen peltatus Ram. in Lam. and DC Fl. Franc. 3rd ed., 2:377. 1805.

Lecanora var. heteromorpha Ach. Lichenogr. Univers. 412. 1810.

Thallus greenish straw-colored or pale brown, squamulose, orbicular, consisting of small closely united groups of squamules, or sometimes of a 
single squamule, with rather entire edges not much lobed; below wrinkled and pitted; attached to the substratum by an umbilicus. Apothecia circular, numerous, when crowded becoming irregular in shape, $1.0-3.0 \mathrm{~mm}$. in diameter, subsessile to sessile; disk grayish green, pale yellow, or fawn color $(R)$, concave to flat; thalline margin concolorous with the thallus, thin and entire or becoming crenate and flexuose, finally disappearing; asci 8-spored; spores hyaline, ellipsoid, nonseptate, 9.0-16.0 x 6.0-8.0 $\mu$.

On rock: Kittitas Co.: Roslyn, $2800 \mathrm{ft.,} \mathrm{1931.} \mathrm{902.} \mathrm{Yakima} \mathrm{Co.} \mathrm{:} \mathrm{gulch}$ near Naches, 1350 ft., 1931, 1141. Okanogan Co. : Tonasket, 1200 ft., 1931, 1363. Columbia Co.: Dayton, 1700 ft., 1940, 2732. Garfield Co. : Pomeroy, $1860 \mathrm{ft} ., 1931,1570$.

The peltate attachment of this lichen makes it very easily recognized. It is rather abundant and widely distributed east of the Cascades.

23. Lecanora rubina (Lam. \& DC.) Ach. Lichenogr. Univers. 412. 1810. Lichen rubinus Lam. in Lam. and DC. Fl. Franc. 3rd ed., $1: 77.1778$.

Thallus yellow, greenish straw colored, or pale brown, thick, squamulose, or subfoliose, much branched at the circumference, short crenate lobed; below dark brown or black; not attached to the substratum by a definite umbilicus. Apothecia circular, 1.0-4.0 mm. in diameter, often clustered, then somewhat irregular in shape, sessile or subsessile; disk pale yellow, fawn color $(R)$, or light reddish brown, flat to convex; thalline margin thin, entire or becoming crenate and flexuous, finally disappearing; asci 8-spored; spores hyaline, ellipsoid, nonseptate, 8.0-16.0 x 5.0-8.0 $\mu$.

On rock: Yakima Co.: Dead Horse Hill, 4500 ft., 1931, 998; Rattlesnake Canyon, 2000 ft., 1931, 1545; gulch near Naches, 1350 ft., 1931, 902a. Klickitat Co.: Bickleton, $3200 \mathrm{ft} .$, 1931, 1661. Douglas Co.: Lower Grand Coulee, 1100 ft., 1931, 1526. Lincoln Co.: Davenport, 2450 ft., 1931, 1507. Washington Territory, Klickitat Co.: Columbia River, 1882, C. J. Sprague 567 (F). Spokane Co.: Spokane, 1907, Bonser (F).

24. Lecanora rubina var. melanophthalma (Ram.) Zahlbr. Cat. Lich. Univ. 5 :659. 1928.

Lichen melanopthalmus Ram. in Lam. and DC. F1. Franc. 3rd ed., $2: 376.1805$.

Thallus very much like the species; disk greenish gray, olivaceous black, or black, often somewhat pruinose; otherwise like the species.

On rock: Yakima Co.: gulch near Naches, $1350 \mathrm{ft} ., 1940,3177$. Chelan Co.: butte near Chelan, 2000.ft., 1931, 1351. Ferry Co. : hillside near Sanpoil, 2000 ft., 1940, 3129. Whitman Co. : Pullman, 2550 ft., 1931, 1584.

25. Lecanora gelida (L.) Ach. Lichenogr. Univers. 429. 1810.

Lichen gelidus Linn. Mantissa 1 :133. 1767.

Thallus light buff $(\mathrm{R})$, pale greenish gray, or dirty white, adnate, chinky, or areolate towards the center, more or less radiate, circumference 
lacinate-lobate, deeply lobed, bearing one large or several small brownish cephalodia and white soredia; yellow with $\mathrm{KOH}$, red with $\mathrm{CaOCl}_{2}$, closely attached to the substratum by hyphal rhizoids. Apothecia yellow, pinkisin buff $(\mathrm{R})$, fawn color $(\mathrm{R})$, or brown, circular, 0.8-12.0 $\mathrm{mm}$. in diameter, adnate; disk fawn color ( $R$ ), often blackening, concave to flat; thalline margin concolorous with the thallus, thick, persistent; asci 8-spored; spores hyaline, ellipsoid, nonseptate, $14.0-20.0 \times 6.0-11.0 \mu$.

On rock: Clallam Co.: Lake Crescent, 483 ft., 1931, 1037. San Juan Co.: Orcas Island, Mt. Constitution, 2500 ft., 1931, 2032. Clallam Co.: Lake Crescent, Storm King Mountain, 3500 ft., 1935, A. H. Smith 2251 (F). King Co.: Summit, 1907, Foster 649 (F).

A very distinctive Lecanora that is easily recognized by the large clusters of cephalodia and abundant soredia.

47. Ochrolechia Mass. Ricerch. Auton. Lich. 30. 1852.

Thallus uniformly crustose, areolate, smooth, warty to rarely minutely fruticose, sometimes sorediate, upper cortex thin or wanting, lower entirely absent, algal layer and medulla distinct. Apothecia circular, medium sized or rather large, immersed, adnate to sessile; proper margin absent, thalline margin present, usually concolorous with the thallus, somewhat irregular in shape; hypothecium hyaline; paraphyses branched and entangled; asci 2-8-spored, spores large, hyaline, ellipsoid or ovoid.

Alga Protococcus.

A. Thallus red with $\mathrm{CaOCl}_{2}$, coralloid branched, disk naked....1. O. tartarea A. Thallus not red with $\mathrm{CaOCl}_{2}$, not coralloid branched, disk pruinose.

2. O. upsaliensis

1. Ochrolechia tartarea (L.) Mass. Ricerch. Auton. Lich. 30. 1852.

Lichen tartareus Linn. Sp. P1. 1141. 1753.

Lecanora tartarea (L.) Ach.

Thallus grayish white or rarely shades of brown, tartareous, wide spread, consisting of granules or warts, at times with coralloid branches; yellow then pink with $\mathrm{KOH}$ and red with $\mathrm{CaOCl}_{2}$. Apothecia medium or large, circular, 1.0-3.0 mm. in diameter, crowded and often twisted out of the normal shape, adnate to sessile; disk wood brown ( $R)$, pale yellow, or brick color, slightly granular, somewhat wrinkled, concave to flat; thalline margin thick, concolorous with the thallus, corrugate and flexuous; asci 2-8-spored; spores hyaline, very large, ellipsoid-oblong, nonseptate, $30.0-62.7 \times 26.4-33.0 \mu$.

On bark and rocks, rarely on soil: Pacific Co.: Loomis Lake, 1930, 432. San Juan Co.: San Juan Island, 1928, 2028; Trout Lake, 1940, 3226. Kitsap Co.: Hidden Ranch near Bremerton, 213 ft., 1930, 533; 1940, 3292. King-Kittitas Co.: Snoqualmie Pass, 3010 ft., 1931, 826. Pierce Co.: Mt. Rainier, Summerland Park, 6500 ft., 1931, 689; Van Trump Park, 5200 ft., 1942, 3609. Yakima Co. : Lodge Pole Pine Camp, 3500 ft., 1940, 3310; 
American River Camp, 2800 ft., 1931, 955. Lincoln Co.: Davenport, 2450 ft., 1931, 1510a.

A very common bark lichen, easily recognized by the large apothecia, the thick, prominent margin, and the granular and often wrinkled disk.

2. Ochrolechia upsaliensis (L.) Mass. Ricerch. Auton. 31. 1852.

Lichen upsaliensis Linn. Sp. P1. 1142. 1753.

Ochrolechia pallescens (L.) Mass.

Thallus white or gray, determinate or effuse, rather thick, of smooth areoles or chinky and plicate, often becoming tuberculate; no color change with $\mathrm{KOH}$ or $\mathrm{CaOCl}_{2}$. Apothecia circular, medium, 0.5-1.5 mm. in diameter, adnate; disk white or yellowish white, densely granular pruinose, concave to flat; thalline margin concolorous with the thallus, thick, prominent, sometimes rough; asci 4-8-spored; spores hyaline, large, ellipsoid, nonseptate, $40.0-60.0 \times 23.0-35.0 \mu$.

Encrusting mosses on soil or rock: San Juan Co.: Shaw Island, 1928, 2029. Kittitas Co.: Roslyn, 2800 ft., 1931, 1798. Ferry Co. : Republic, 2650 ft., 1931, 1405; Gibraltar Mt., 3783 ft., 1940, 3161. Whitman Co. : Pullman, $2550 \mathrm{ft} ., 1931,1581$. Olympic Mountains, Boulder Peak, $6000 \mathrm{ft} ., 1935$, A. H. Smith 2910 (F). Chelan-Kittitas Co.: Blewett Pass, 1919, J. M. Grant (WTU).

Often found encrusting Selaginella as well as mosses, this, its most frequent habitat makes it an easy lichen to know.

48. Icmadophila Trevis. in Revista Periodic. Lavori I. R. Accad. Padova. 267. 1851-52.

Thallus crustose, no differentiation into definite layers. Apothecia circular, often somewhat angular, sessile to short stipitate, both proper and thalline margins present, thalline margin soon disappearing; hypothecium hyaline; paraphyses unbranched, and separate; asci cylindrical; 8-spored, spores hyaline, fusiform, 1-3-septate.

Alga Protococcus.

1. Icmadophila ericetorum (L.) Zahlbr. in Wiss. Mitteilung. Bosnien and Hercegov. $3: 605.1895$.

Lichen ericetorum Linn. Sp. P1. 1141. 1753.

Baeomyces aeruginosus (Scop.) DC.

Thallus greenish gray, silver gray, tea green (R), or olive-buff (R), very thin and smooth, resembling a layer of paint, or thick and rough, forming a granulose or warty crust, rarely tartareous. Apothecia circular or angular in shape, medium or large, $0.5-3.0 \mathrm{~mm}$. in diameter, adnate to sessile or substipitate; disk pale flesh color ( $R$ ), or rosy pink, concave, flat or convex, wrinkled; proper margin thin, thalline margin concolorous with the thallus, often disappearing; spores hyaline, oblong-fusiform, 1-3-septate, $18.4-30.0 \times 4.0-6.0 \mu$.

Encrusting mosses and old logs: Pacific Co.: Oysterville, 1931, 750. San Juan Co.: Orcas Island, Mt. Constitution, 2500 ft., 1931, 2031. Clallam 
Co. : Cedarville, 300 ft., 1940, 2892. Kitsap Co.: Hidden Ranch near Bremerton, 213 ft., 1931, 529. King Co.: Grass Mt. Trail, 2200 ft., 1931, 711. Whatcom Co.: Mt. Baker, 1000 ft., 1914, G. Turesson (W). Thurston Co.: Gate, 1912, Foster 1984 (W). Ferry Co.: Republic near Sanpoil River, Foster (W).

A beautiful lichen, usually found growing in dense woods on decaying logs, easily recognized because of the pinkish colored disks of the apothecia on the greenish gray thallus.

49. Lecania Mass. Alcun. Gen. Lich. 12. 1855.

Thallus uniformly crustose, sometimes marginally lobed, rarely dwarffruticose, no definite division into layers; no color change with $\mathrm{KOH}$. Apothecia circular, often crowded, then angular, adnate to sessile; proper margin rarely appearing, thalline margin present; hypothecium hyaline; paraphyses unbranched, distinct; asci normally 8-spored, sometimes 16-32spored; spores hyaline, fusiform or ellipsoid, 1-many-septate.

Alga Protococcus.

1. Lecania cyrtella (Ach.) Th. Fr. Lichenogr. Scand. $1: 294$. 1871-74.

Lecidea cyrtella Ach. Meth. Lich. 67. 1803.

Biatora cyrtella (Ach.) Mann.

Thallus white, grayish green, pale yellow, or dark brown, very thin, smooth or becoming warty, sometimes disappearing. Apothecia circular, angular when crowded, $0.2-0.8 \mathrm{~mm}$. in diameter, adnate to subsessile; disk pale flesh color ( $R)$, tawny, or blackish, flat to convex, biatorine; thalline margin entire, disappearing early; asci 8-16-spored; spores hyaline, fusiform-oblong, 1-3-septate, 9.0-16.2 x 4.6-5.0 $\mu$.

On bark: San Juan Co.: Turn Island, 1928, 2026. Ferry Co.: Republic, 1913, Foster 2419 (F). Washington Territory, Suksdorf (F).

50. Haematomma Mass. Ricerch. Auton. Lich. 32. 1852.

Thallus uniformly crustose or squamulose, tartareous, thick, rough or smooth, with a more or less well-developed upper cortex, other layers not well developed. Apothecia circular, sessile; epithecium blue with $\mathrm{KOH}$; proper margin absent, thalline margin concolorous with the thallus, thin and soon disappearing; hypothecium hyaline; paraphyses unbranched, thread like, brownish and slightly thickened at the tips; asci 8-spored; spores hyaline, finger, needle, or spindle form, straight or curved, 3-many-septate, thin walled with cylindrical cells.

Alga Protococcus.

1. Haematomma ventosum (L.) Mass. Ricerch. Auton. Lich. 33. 1852.

Lichen ventosus Linn. Sp. P1. 1141. 1753.

Lecanora ventosa (L.) Ach.

Thallus pale yellow, dirty yellow, greenish yellow, or gray, tartareous, consisting of cracked areoles, thick; yellow with $\mathrm{KOH}$. Apothecia circular, 
medium to large, $1.0-4.0 \mathrm{~mm}$. in diameter, adnate; disk blood red, haematite red $(R)$, naked, epithecium deep purple; blue with $\mathrm{KOH}$, flat to convex; thalline margin concolorous with the thallus, rough, crenate, thin and soon disappearing; spores hyaline, needle shaped, curved, 3-7-septate, 33.0-50.0 x 3.0-5.0 $\mu$.

On rock: Fink (1935) reports it from Washington on rocks and trees.

51. Candelariella Müll. Arg. in Bull. Herb. Boiss. 2 :app. 1. 11, 47. 1894.

Thallus uniformly crustose or squamulose, granular or warty, sometimes with lobed margin, no definite division into layers; no color change with $\mathrm{KOH}$ or $\mathrm{CaOCl}_{2}$. Apothecia circular, sessile, yellow; no color change with $\mathrm{KOH}$; proper margin lacking; thalline margin well developed; hypothecium hyaline; paraphyses unbranched, distinct, nonseptate or septate towards the tips and jointed, asci 8-many-spored, spores hyaline, oblong or ellipsoid, nonseptate, or 1-septate.

Alga Protococcus.

A. Thallus granulose, sometimes disappearing.

1. C. aurella

A. Thallus usually squamulose, continued or scattered, never disappearing.

2. C. vitellina

1. Candelariella aurella (Hoffm.) Zahlbr. Cat. Lich. Univ. 5:790. 1928. Verrucaria aurella Hoffm. Deutschl. F1. 197. 1796.

Placodium vitellinum var. aurellum (Hoffm.) Tuck.

Candelariella cerinella Zahlbr.

Thallus dark gray, greenish brown, or black, inconspicuous, tartareous, granulose, often scattered, sometimes disappearing. Apothecia 0.5-0.8 mm. in diameter, adnate to sessile; disk greenish yellow or olivaceous, flat to convex, thalline margin thin, entire, to slightly granulose-crenate; asci more than 8-spored; spores hyaline, ellipsoid, nonseptate, or 1-septate, 13.2-16.5 x 3.6-4.9 $\mu$.

On rock, old wood, or soil: Chelan Co.: Zena, $800 \mathrm{ft.,} 1931,1107 a$. Klickitat Co.: Goldendale, 3000 ft., 1931, 1701. Ferry Co.: Republic, Maye's Butte, 1913, Foster 2388 (F).

2. Candelariella vitellina (Ehrh.) Müll. Arg. in Bull. Herb. Boiss. 2 : app. 1. 47.1894.

Lichen vitellinus Ehrh. P1. Crypt. Exsicc. No. 155. 1785.

Placodium vitellinum (Ehrh.) Hepp.

Thallus bright yellow or greenish gray, usually squamulose, squamules continuous or scattered, crenate lobed, rather small, sometimes granulose, granules often heaped and crowded. Apothecia 0.5-1.2 $\mathrm{mm}$. in diameter, sessile ; disk yellow, tawny yellow, or olivaceous, flat; thalline margin concolorous with the thallus, granulate-crenate; asci 12-32-spored, rarely 8-spored; spores hyaline, ellipsoid, nonseptate or 1-septate, $9.9-16.5 \mathrm{x}$ 3.6-5.9 $\mu$. 
On soil, rock, old wood, or bark: Kittitas Co.: Easton, 2168 ft., 1931, 843a. Yakima Co.: American River Camp, 2800 ft., 1931, 951. Klickitat Co.: Bickleton, 3200 ft., 1931, 1638. Chelan Co.: butte near Chelan, 2000 ft., 1931, 1323. Ferry Co.: Sanpoil, 2360 ft., 1940, 3131. Spokane Co.: Mt. Spokane, 5808 ft., 1931, 1501. Clallam Co.: Lake Crescent, Storm King Mountain, 3500 ft., 1935, A. H. Smith 2236 (F).

A common soil and rock lichen east of the Cascades; it is easily recognized by the bright yellow color of its small crowded squamules.

\section{PARMELIACEAE}

Thallus foliose, rarely subfruticose or fruticose, dorsi-ventral, ascending, differentiated into a typical or a loose and poorly developed plectenchymatous upper cortex, a typical plectenchymatous lower cortex, algal, and medullary layers, attached to the substratum by rhizoids, or rhizoids entirely lacking. Apothecia circular, scattered, marginal or terminal, sessile or subpedicellate; proper margin absent, thalline margin well developed and prominent; asci 2-8-spored, rarely 16-many-spored; spores hyaline, nonseptate, or rarely becoming 1-septate. Spermatia are borne laterally (endobasidial) or rarely terminally (exobasidial).

Alga Protococcus.

A. Upper cortex typically plectenchymatous, spermatia lateral.

B. Thallus more or less erect; apothecia marginal or terminal.

C. Apothecia originating on the lower surface, but by the turning of the lobes they appear to grow on the upper surface.

56. Nephromopsis

C. Apothecia originate on the upper surface.

55. Cetraria

B. Thallus usually flat or depressed; apothecia scattered on the upper surface.

D. Asci multisporous.

52. Candelaria

D. Asci rarely more than 8 -spored.

54. Parmelia

A. Upper cortex loosely and irregularly plectenchymatous, spermatia

terminal.

53. Parmeliopsis

52. Candelaria Mass. in Flora. 35 :567. 1852.

Thallus foliose, small, often very small, laciniately divided, differentiated into typical upper and lower plectenchymatous cortices, thin algal and medullary layers, attached to substratum by short rhizoids; no color change with $\mathrm{KOH}$. Apothecia small, scattered, sessile; thalline margin prominent; hypothecium hyaline, to pale brown; paraphyses rarely branched; asci 16 or more-spored; spores hyaline, nonseptate, or rarely becoming 1-septate, spermatia lateral. This genus resembles Caloplaca, but the lack of color change of the thallus with $\mathrm{KOH}$ readily separates it from Caloplaca.

1. Candelaria concolor (Dicks.) Arn. Flora 62:364. 1897.

Lichen concolor Dicks. Fasc. P1., Crypt. Brit. 3:18, tab. 9. 1793.

Teloschistes concolor (Dicks.) Tuck. Syn. N.Am. Lich. 1:51. 1882. 
Thallus greenish yellow ( $R$ ), bright yellow, or ashy white, small, suborbicular or spreading, lobes small, laciniate crenate, or somewhat imbricated, often forming a continuous crust, near the center, granulose, sorediate, or isidiose; below pale, ashy, with rhizoids. Apothecia very small, scattered, circular, $0.2-0.8 \mathrm{~mm}$. in diameter, sessile; disk orange, yellow, or yellowish brown, concave to flat; thalline margin concolorous with the thallus, entire to slightly granulate; hypothecium hyaline; asci 20-60spored; spores hyaline, oblong, nonseptate, or imperfectly 1-septate, 9.3-12.4 × 2.0-4.5 $\mu$.

On trees, stumps, or rock: Kittitas Co.: South Cle Elum, 3000 ft., 1931, 860. Chelan Co.: Merritt, $2186 \mathrm{ft} ., 1931,1085 a$; butte near Chelan, 2000 ft.. 1931, $1326 a$.

53. Parmeliopsis Nyl., Syn. Lich. 2 :53. 1863.

Thallus foliose, small, thin, appressed, upper cortex loosely and irregularly plectenchymatous, lower cortex typical and well developed, rather irregular algal and medullary layers; attached to the substratum by scattered rhizoids. Apothecia rare, small to medium sized, scattered, sessile; thalline margin present; hypothecium hyaline to pale brown; paraphyses rarely branched, asci 8-spored; spores hyaline, straight or curved, nonseptate. Spermatia terminal.

A. Thallus usually yellowish, with sulphury soredia, often broken in the center, forming a powdery mass.

1. P. ambigua

A. Thallus grayish white to grayish olive, with concolorous soredia.

2. P. diffusa

1. Parmeliopsis ambigua (Wulf.) Nyl. Syn. Lich. 2:54. 1863.

Lichen ambiguus Wulf. in Jacq. Coll. Bot. 4:239. 1790.

Parmelia ambigua (Wulf.) Ach. Meth. Lich. 207. 1803.

Thallus naphthalene yellow $(R)$, straw yellow $(R)$, very pale yellow, or grayish white, opaque, stellate, adnate, lobes linear, much branched dichotomously, margins wavy to crenate, tips rounded, smooth near the edge, rough and broken in the center, more or less densely yellow or white sorediate, center is often in the form of an indefinite powdery mass; below brown to black with scattered black rhizoids. Apothecia rare, 1-4 mm. in diameter; disk pale brown or chestnut brown, concave to flat; thalline margin concolorous with the thallus, crenulate; spores hyaline, usually curved, oblong-ovoid, nonseptate, $6.0-13.0 \times 2.5-3.3 \mu$.

On trees, decaying wood, or rock: King-Kittitas Co.: Snoqualmie Pass, $3010 \mathrm{ft} .$, 1931, 821. Kittitas Co. : Easton, $2186 \mathrm{ft} ., 1931$, 845. Yakima Co. : Deadhorse Hill, 4500 ft., 1931, 981a. Ferry Co.: Gibraltar Mt. near Republic, 3100 ft., 1940, 3155. Okanogan Co.: Twisp Trail, 4000 ft., 1931, 1300. Ferry Co.: Laurier, 164 ft., 1931, 1446. Whitman Co.: Tekoa Mountains, 2900 ft., 1931, 1600. Columbia Co.: Blue Mountains, 3500 ft., 1931, 1626.

A small and inconspicuous lichen. The decided yellow color separates it readily from Parmeliopsis diffusa, the only other species found in the state. 
2. Parmeliopsis diffusa (Web.) Riddle Bryol. 20:75. 1917.

Lichen diffusus Web. Spicil. Fl. Goet. 250. 1778.

Parmelia ambigua var. albescens (Wahl.) Schaer. Enum. Lich. Eur. 47. 1850.

Thallus whitish to cinereous (R) or grayish olive (R), suborbicular, closely adnate, smooth to even shiny near the edges, lobes linear, radiate, much branched dichotomously, margins crenate, tips rounded, rimulose and broken towards the center, with raised balls of white sometimes grayish soredia, crowded towards the center, never isidiose; below brownish, black with scattered black rhizoids. Apothecia rare, $2-6 \mathrm{~mm}$. in diameter; disk pale brown to chestnut brown, concave to convex; thalline margin concolorous with the thallus, crenulate; asci 8 -spored; spores hyaline, straight or curved, oblong, nonseptate, 7.0-10.0 x 2.0-3.3 .

On trees and decaying wood: Pierce Co. : Silver Springs, 2670 ft., 1931, 721; White River Camp, 3900 ft., 1940, 3103; Mt. Rainier, Paradise Park, 5550 ft., 1928, 1921; Berkeley Park, 6000 ft., 1940, 3020. Pierce-Yakima Co.: Chinook Pass, 5440 ft., 1559. Kittitas Co.: Easton, 2168 ft., 1931, 926. Olympic Mountains, Camp Elkhorn, 2000 ft., 1907, Frye 73 (F), this specimen determined as Parmelia hyperopta by G. K. Merrill. Ferry Co.: Republic, 1912, Foster 2292 (F), this specimen determined as Parmelia diffusa var. albescens by G. K. Merrill.

Riddle (1917) has been the basis for the treatment of this genus.

\section{Parmelia Ach. Meth. 153. 1803.}

Thallus foliose, rarely subfruticose, variously lobed, smooth, sorediate, or isidiose, sometimes ciliate on the margins, well-developed upper and lower plectenchymatous cortices, also medullary and algal layers, lower surface either lighter or darker than the upper, with or without rhizoids. Apothecia scattered on the upper surface, never marginal, shield-shaped (scutellate), sessile or subpedicellate, thalline margin prominent; hypothecium hyaline; paraphyses usually branched, septate, embedded in a jell and enlarged at the tips; asci commonly 8-spored, rarely more numerous; spores hyaline, nonseptate.

A. Subfruticose, black or very dark brown.

B. Lobes filamentous. 12. P. pubescens

B. Lobes narrowly membranaceous, never more than $1 \mathrm{~mm}$. wide.

A. Foliose.

13. P. stygia

C. Never bearing rhizoids below.

D. Thallus perforated with occasional small holes. 7. P. pertusa

D. Thallus never perforated with small holes.

E. Cribrose below. 3. P. lophyrea

E. Not cribrose below.

F. Lobes much inflated, usually elongated, black spermagonia often abundant. 
G. Black margin and black underside, very conspicuous, lobes lax, often sharp pointed................................... $P$. vittata

f. Black margin not conspicuous, lobes shorter, tips rounded ventricose.

H. Lobes rugose, twisted, and crowded, very broad.

2. P. enteromorpha f. rugosa

$\mathrm{H}$. Lobes not rugose, twisted, and crowded, medium width.

1. P. enteromorpha

F. Lobes only slightly inflated, irregularly branching, tips usually ascending, black spermagonia not abundant.

I. Not always sorediate.

4. P. physodes

I. Always sorediate, lobes shaped much like a trumpet, open and filled with soredia.

5. P. tubulosa

C. Always bearing rhizoids below.

J. Some shade of real brown, often olive-brown.

$\mathrm{K}$. Apothecia always numerous, asci more than 8-spored.

16. P. multispora

$\mathrm{K}$. Apothecia not always numerous, asci never more than 8-spored.

L. Marginal lobes rimose-reticulate, center thickly beset with coralloid branchlets.

M. Below light colored, color of medulla unchanged with $\mathrm{P}$.

M. Below dark colored, medulla red with $\mathrm{P}$.

19. P. saxatilis var. Aizoni

L. Not as above.

N. Bearing coralloid branchlets or powdery soredia.

O. Lobes narrow, always longer than wide, usually on rocks.

11. P. prolixa

O. Lobes broad, always wider than long, usually on wood.

9. P. olivacea var. aspidota

N. Never bearing coralloid branchlets, isidia, or soredia.

P. Lobes narrow, crowded, imbricated...10. P. pannariiformis

P. Lobes broad, always wider than long, not imbricated.

8. P. olivacea

J. Some color other than brown.

Q. Reticulate-rimose.

$\mathrm{R}$. Conspicuously sorediate on the reticulations; also often on the margins.

20. P. sulcata

R. Rarely if ever sorediate.

$\mathrm{S}$. Very sparingly isidiose.

18. $P$. saxatilis

S. Very densely isidiose.

T. Below light colored, color of medulla unchanged with $P$.

T. Below dark colored, medulla red with $P$.

17. P. rudecta

19. P. saxatilis var. Aizoni

Q. Never reticulate-rimose.

U. Margins bearing clustered sorediate lobules. 
V. Margins conspicuously ciliate........24. P. perlata var. ciliata

V. Margins not conspicously ciliate. 23. P. perlata

U. Margins not bearing clustered sorediate lobules.

W. Below very dark brown or black, edges lighter, rhizoids abundant at the center, lacking or very sparse on the margins, upper cortex not red with $\mathrm{P}$.

$\mathrm{X}$. Margins always ciliate. 22. P. crinita

$\mathrm{X}$. Margins never ciliate. 21. P. caperata

W. Below lighter colored, rhizoids evenly distributed, upper cortex red with $\mathrm{P}$.

Y. Upper surface smooth, usually on rocks...14. P. conspersa

Y. Upper surface isidiose or sorediate.

15. $P$. conspersa var. isidiata

1. Parmelia enteromorpha Ach. Meth. Lich. 252. 1803.

Parmelia physodes var. enteromorpha Tuck. Proc. Am. Acad. Arts and Sci. $1: 220.1848$.

Thallus ivory yellow (R), deep olive-gray ( $R)$, honey yellow (R), water green $(R)$, deeply divided into rather broad, thick and inflated, wavy lobes, complicate, abundantly sprinkled with black spermagonia (appearing like black dots); below dark reddish brown to black, brown at the tips, smooth, often shiny, much wrinkled, often with holes in the lower cortex, rhizoids absent. Apothecia rather abundant, medium sized to large, sometimes very large, $3-15 \mathrm{~mm}$. in diameter, subpedicellate, ventricose ; disk reddish brown, deeply concave, finally becoming convex; thalline margin entire to crenate; asci 8-spored; spores hyaline, ellipsoid, nonseptate, 4.0$8.0 \times 3.0-6.0 \mu$.

On bark, sticks, and dead branches: San Juan Co.: San Juan Island, Pt. Caution, 1928, 2039. Pacific Co.: Loomis Lake, 1930, 431. King Co.: Richmond Highlands, 200 ft., 1931, 579. King-Kittitas Co.: Snoqualmie Pass, 3010 ft., 1931, 814. Pend Oreille Co.: Metaline Falls, 2600 ft., 1931, 1482. Whitman Co.: Tekoa Mts., 2900 ft., 1931, 1599. Olympic Mountains, Camp Elkhorn, 1800 ft., 1907, Frye (F). Island Co. : Langley, 1920, J. M. Grant 3036 (F). Pierce Co.: Rainier National Park, 1923, J. M. Grant 430 (F). Spokane Co.: Spokane, 1932, Sister Marion Loretta (WTU).

One of the common lichens, widely distributed in the state; the abundant black spermagonia a good distinguishing character.

2. Parmelia enteromorpha f. rugosa (Merrill) Zahlbr. Cat. Lich. Univ. $6: 33.1930$.

Parmelia physodes var. enteromorpha f. rugosa Merrill, Bryol. 11 :86. 1908.

Thallus pale yellow orange to ivory yellow $(R)$, differs from the species in having larger, much more inflated lobes, that are rugose and twisted and crowded. 
On bark: Pierce Co. : Silver Springs, 2670 ft., 1931, 722 ; Mt. Rainier, Emmons Glacier Trail, 5000 ft., 1931, 661. Yakima Co.: Deadhorse Hill, $4500 \mathrm{ft} ., 1931,981$. Plate V, A.

3. Parmelia lophyrea Ach. Meth. Lich. 198. 1803.

Thallus ivory yellow ( $R$ ) or deep olive-buff ( $R$ ), cartilaginous, flat, thin, pitted, lobes sinuately many clefted, rather short, tips deeply retuse to truncate; below dark brown to black, cribrose, smooth with no rhizoids. Apothecia medium to rather large sized, $2-8 \mathrm{~mm}$. in diameter, sessile to subpedicellate; disk Dresden brown $(R)$ or bay (R), folded, sinuose, flexuose; thalline margin entire to subcrenate; asci 8 -spored; spores hyaline, globose, nonseptate, 3.6-4.0 $\mu$ in diameter.

On trees: Pacific Co.: Loomis Lake, 1931, 740a; 1940, 2849. Wahkiakum Co.: Cathlamet, 1940, 2913. Grays Harbor Co.: Westport, 1908, Foster 724 (F) ; Copalis, Foster 1911 (W). West Coast of North America, Menzies ( $\mathrm{T}$ ).

4. Parmelia physodes (L.) Ach. Meth. Lich. 153. 1803.

Lichen physodes Linn. Sp. P1. 1144. 1753.

Thallus pale olive-gray $(R)$, chamois $(R)$, or ivory yellow $(R)$, suborbicular, flat and spreading, rather loosely attached to the substratum, slightly inflated, irregularly divided into numerous narrow wavy lobes, often becoming crowded in the center, the recurved ascendent tips often abundantly sorediate below, on many of the lobes the black or brown under surface shows as a border; below black to reddish brown, often decidedly brown at the tips, smooth, sometimes wrinkled, often shiny, rhizoids absent. Apothecia rare, $3-8 \mathrm{~mm}$. in diameter, sessile to subpedicellate; disk reddish brown, concave; thalline margin entire to crenate; asci 8 -spored; spores hyaline, sub-ellipsoid, nonseptate, 4.0-7.3 x 3.3-5.2 $\mu$.

On trees, dead branches, decaying logs, or rock: Pacific Co.: Chinook, 1931, 796. Mason Co.: Shelton, 1931, 1223. Kitsap Co.: Hidden Ranch, near Bremerton, 213 ft., 1940, 3286. Snohomish Co.: Chase Sphagnum Bog, 1940, 3334. Yakima Co.: Rattlesnake Canyon, 2000 ft., 1931, 1538. Ferry Co.: Boyds, 1470 ft., 1931, 1438. Columbia Co. : Blue Mts., 3500 ft., 1931, 1623. Clallam Co.: Port Angeles, 1914, Foster 2646 (F); Sequim, 1915, J. M. Grant 213 (F).

A common species, widely distributed, easily distinguished by the dilated, ascendant, recurved tips, that are often sorediate on the under surface. Plate IV, D.

5. Parmelia tubulosa (Schaer.) Bitter, Hedwigia $40: 179.1901$.

Parmelia ceratophylla var. tubulosa Schaer. Lich. Helvet. Spicil. sect. 10. 459.1840.

Thallus is much like Parmelia physodes but the lobes are curved, erect and remarkably tubular, shaped much like a trumpet, the open ends filled with soredia. Apothecia very rare, sterile in this state. 
On trees, dead branches, and decaying wood: King Co.: Edgewood, 300 ft., 1940, 3089. Kittitas Co. : Easton, 2168 ft., 1931, 846. Whitman Co. : Tekoa Mts., 2900 ft., 1931, 1604. Plate V, C.

6. Parmelia vittata (Ach.) Röhl. Deutschl. Fl. 3, Abt. 2:109. 1813.

P. physodes var. vittata Ach. Meth. Lich. 250. 1803.

Thallus grayish olive (R), ivory yellow (R), or pale olive-gray (R), dichotomously divided into long narrow, often quite delicate, slightly inflated lobes, tips rather sharp pointed, often divaricately branched, black margin, conspicuous; below reddish brown to black, often brown at the tips, wrinkled, shiny, rhizoids absent. Apothecia very similar to those of $P$. enteromorpha in both external and internal structure, the disk is usually not so wide.

On trees, dead branches, decaying wood: Pacific Co.: Long Beach, 1928, 1909. Kitsap Co. : Hidden Ranch, near Bremerton, 213 ft., 1928, 523. San Juan Co.: Orcas Island, Mt. Constitution, 2500 ft., 1928, 2044. Pierce Co.: White River Camp, 3900 ft., 1940, 3097. Whatcom Co.: Mt. Baker, Galena, 3800 ft., 1931, 1180. Chelan Co.: Stehekin, 1140 ft., 1931, 1289. Spokane Co. : Mt. Spokane, 5808 ft., 1931, 1489. Grays Harbor Co.: Westport, 1919, J. M. Grant (F) ; Montesano, 1919, J. M. Grant 3011 (F). Klickitat Co.: Goldendale, 1909, Foster 1162 (F). Plate V, B.

A common species, widely distributed, distinguished by the long, slender, black bordered lobes, with forked tips.

7. Parmelia pertusa (Schrank) Schaer. Lich. Helvet. Spicil. sect. 10, 457. 1840.

Lichen pertusus Schrank, Baierisch. Flora 2:519. 1789.

Thallus light grayish-olive or ivory yellow, orbicular, medium sized, closely adnate, slightly inflated, divided palmately into many sinuate lobes, tips rounded, perforated with small rounded holes and beset with conspicuous, raised, round masses of soredia; below black, sometimes brown on tips of lobes, wrinkled, rhizoids absent. "Apothecia very rare except in austral regions, smallish, chestnut, with an entire margin. Spores in twos and fours, ellipsoid, 45-60 × 22-28 $\mu$." From Tuck. Syn. N. Am. Lich. 61. 1882.

On trees or rock: Pacific Co.: Loomis Lake, 1931, 733; Chinook, 1940, 2833. Wahkiakum Co.: Cathlamet, 1940, 2921. Grays Harbor Co.: Aberdeen, 1908, Foster 916 (W) ; Montesano, 1919, J. M. Grant 3030 (F). Thurston Co.: Gate, 1911, Foster 1936 (W). Wahkiakum Co.: Cathlamet, 1907, Foster 467 (W).

Rather a rare species, collected only in the Pacific Coastal Plain, Olympic Mountains, and Puget Sound Basin. The small holes perforating the thallus are a very good distinguishing character. I have seen no fruiting material from the state. 
8. Parmelia olivacea (L.) Ach. Meth. Lich. 213. 1803.

Lichen olivaceus Linn. Sp. P1. 1143. 1753.

Thallus olive-brown ( $R$ ), burnt umber $(R)$, Dresden brown $(R)$, or tawny olive $(R)$, small to medium sized, membranaceous, closely adnate, flat, shiny, smooth or sometimes pitted and rugose, margins often elevated and shiny, lobes radiate, imbricate, rather broad with rounded tips; below dark brown to black, margins lighter, with scattered, short, concolorous, rhizoids. Apothecia usually abundant, 2-6 $\mathrm{mm}$. in diameter, sessile to subsessile; disk dark reddish brown, concave to flat, thalline margin entire or crenulate; asci 8-spored; spores hyaline, ovoid-ellipsoid, thick-walled, nonseptate, $7.0-15.0 \times 6.0-8.0 \mu$.

On bark: Island Co.: Langley, 1923, J. M. Grant. Wahkiakum Co.: Cathlamet, 1906, Foster (F). Pierce Co.: Longmire Springs, 2761 ft., 1906, Harper and Harper (F). Thurston Co.: Gate, 1912, Foster 2077 (W). Whitman Co.: Pullman, 1900, Beattie 32 (F).

This species was collected only a few times, stations widely separated.

9. Parmelia olivacea var. aspidota Ach. Meth. Lich. 214. 1803.

Lichen aspidotus Hoffm. Enum. Lich. 1784.

Parmelia aspidota (Ach.) Röhl. Deutschl. Fl. 3, Abt. 2:100. 1813.

Thallus always abundantly isidiose, sometimes sorediate, otherwise like the species. Apothecia not abundant. Followed Berry's classification (1941).

On bark, rarely on rocks: Pacific Co.: Loomis Lake, 1931, 738. San Juan Co. : Friday Harbor, 1928, 2045. Wahkiakum Co.: Cathlamet, 1940, 2915. Thurston Co.: Grand Mound, 1940, 2950. King Co. : Edgewood, 300 ft., $1940,3087$.

This variety is not widely distributed.

10. Parmelia pannariiformis (Nyl.) Wainio, Arkiv. för Bot. 8: no. $4: 28$. 1909.

Parmelia olivacea var. panniformis Nyl., Herb. Muscei Fenn. 83. 1859. Parmelia olivacea var. prolixa f. panniformis Th. Fr.

Thallus dark olive-brown ( $R$ ) or olivaceous black (R), looks curly or crisp, forming a flat mat divided into tiny lobes, irregularly cut, densely crowded and imbricated, often with finger like branches; below bearing scattered rhizoids, closely attached to the substratum. Apothecia rare; I have not found it fruiting in the state. (Apothecia 2-4 $\mathrm{mm}$. in diameter, sessile; disk dark reddish brown, concave to flat; thalline margin crenulate or dentate; asci 8-spored; spores hyaline, ovoid-ellipsoid, nonseptate, "9.0$12.0 \times 4.0-7.0 \mu$.") This description was made from a specimen collected at Gate of Notch, White Mountains, 1866, by Tuckerman, determined by him as $P$. olivacea var. prolixa $\mathrm{f}$. panniformis $\mathrm{Nyl}$.

On rock: Chelan Co.: Rainbow Trail, 3500 ft., 1931, 1248; Merritt, 2186 ft., 1931, 1089. Ferry Co. : Laurier, 1644 ft., 1931, 1417. 
This is a small inconspicuous Parmelia, which may account for the few collections made.

11. Parmelia prolixa (Ach.) Röhl. Deutschl. Fl. 3, Abt. 2:100. 1813.

Parmelia olivacea var. prolixa Ach. Meth. Lich. 214. 1803.

Thallus dusky drab $(R)$, olive-brown $(R)$, or blackish brown $(R)$, orbicular to suborbicular, loosely adnate, divided into short or often long, narrow, crowded, rough and rugose or smooth and shiny, radiating lobes, often bearing white or brown coralloid branches, margins wavy or crenate, usually ascending, smooth and shiny; below dark brown or black, bearing black rhizoids. Apothecia 1-6 mm. in diameter, sessile; disk reddish brown or blackish brown $(R)$, concave to flat; thalline margin entire or subcrenate; asci 8-spored; spores hyaline, ellipsoid, nonseptate, 7.0-14.1 x 5.0-8.0 $\mu$.

Usually on rocks, rarely on bark: San Juan Co. : San Juan Island, Pt. Caution, 1928, 1917. Chelan Co. : butte near Chelan, $2000 \mathrm{ft} ., 1931,1329$. Okanogan Co.: Brewster, 2450 ft., 1931, 1467. Lincoln Co.: Davenport, 2450 ft., 1931, 1504. Garfield Co. : Pomeroy, 1860 ft., 1940, 2737. Spokane Co.: Spokane, 1929 ft., 1940, 2749. Clallam Co.: Sequim, 1916, J. M. Grant 558 (F). Walla Walla Co.: Wallula, 1906, Foster 298 (F).

This species is widely distributed, but not very abundant anywhere. The numerous coralloid branches, which are often broken, exposing the white medulla, are a good distinguishing character.

\section{Parmelia pubescens (L.) Wainio, Meddel. Soc. Fauna \& Fl. Fenn. $14: 22.1888$.}

Lichen pubescens L. Sp. P1. 1155. 1753.

Parmelia lanata (L.) Wallr.

Thallus subfruticose, blackish brown (R) or fuscous-black (R), slender, terete, somewhat flattened filaments, rather irregularly dichotomously branched, decumbent, sometimes shiny; below somewhat lighter, attached to the substratum by short, thick, disk-like holdfasts, rhizoids small and scarce. Apothecia 1-5 mm. in diameter, sessile; disk concolorous or black, flat; thalline margin entire or granulose; asci 8-spored; spores hyaline, globose to ellipsoid, nonseptate, 6.0-10.0 x 4.0-6.0 $\mu$.

On rock, on a thin layer of soil over rock, or bark: Pierce Co.: Mt. Rainier, Paradise Park, 5550 ft., 1928, 1874a; Yakima Park, 6300 ft., 1931, 598. Klickitat Co.: Goldendale, $2000 \mathrm{ft} .$, 1931, 1686. Chelan Co. : Rainbow Trail, 3500 ft., 1931, 1247. Whatcom Co.: Ruth Mt., 5500 ft., 1928, L. Sunquist 1902. Mt. Rainier, 1925, J. M. Grant 404 (F). Klickitat Co.: Goldendale, on Steptoe Butte, 1909, Foster 1179 (F). Ferry Co. : Republic, Maye's Butte, 1913, Foster 2400 (F).

An interesting alpine lichen, not found below $2000 \mathrm{ft}$., collected in many rather widely separated mountainous regions of the state. Its resemblance 
to small mats of tangled hair makes it an easy species to recognize in the field.

13. Parmelia stygia (L.) Ach. Meth. Lich. 203. 1803.

Lichen stygius Linn. Sp. P1. 1143. 1753.

Thallus black or fuscous-black ( $R)$, small, subfruticose, shiny, depressed or procumbent, palmately or irregularly branched, lobes flat or convex, very narrowly linear, intricately twisted, often becoming terete, forming imbricated crust-like masses, tips curved under, loosely attached to the substratum; below black, with short rhizoids. Apothecia flexuous, 2-6 $\mathrm{mm}$. in diameter, sessile; disk dark brown or black, slightly concave to flat; thalline margin entire or slightly granular; asci 8-spored; spores hyaline, ellipsoid, nonseptate, 7.0-9.0 × 5.0-6.0 $\mu$.

On rocks : Pierce Co. : Mt. Rainier, Burroughs Mountain, 7300 ft., 1931, 607; Tatoosh Range, Pinnacle Peak, 6562 ft., 1909, Foster 1055 (F).

A rare species collected only in the high mountains.

\section{Parmelia conspersa (Ehrh.) Ach. Meth. Lich. 205. 1803.}

Lichen conspersus Ehrh. in Ach. Lich. Suec. Prodro. 118. 1798.

Thallus grayish-olive ( $R$ ), straw yellow (R), or greenish-straw yellow $(\mathrm{R})$, membranaceous, at first orbicular, finally widely spreading and very irregular in shape, adnate, smooth and shiny, or somewhat wrinkled and dull, lobes long and narrow, imbricated, pinnatified, dichotomously or irregularly branched, lobes long and narrow, arising from the center, forming irregular elevated heaps, margins entire or crenate, tips of lobes appressed and dilated; below pale to purplish-brown, rarely black, with short black, evenly distributed rhizoids. Apothecia abundant, $2-8 \mathrm{~mm}$. in dimargin incurved, crenulate; asci 8-spored; spores hyaline, ellipsoid, nonseptate, 7.5-12.0 x 4.5-6.0 $\mu$. Upper cortex reddish brown with paraphenylenediamine.

Usually on rock, rarely on wood: Clallam Co.: San Juan Island, Friday Harbor, 1931 2107; Orcas Island, Mt. Constitution, 2500 ft., 1928, 2046. Chelan Co.: White Rock Camp, 2100 ft., 1931, 1055; Rainbow Trail, $3500 \mathrm{ft}$., 1931, 1251. Ferry Co.: Mt. Gibraltar near Republic, $3100 \mathrm{ft}$., 1940, 3169. Garfield Co.: Pomeroy, 1860 ft., 1940, 2736. Whitman Co.: Steptoe Butte, 3700 ft., 1940, 3333. San Juan Co. : San Juan Island, Friday Harbor, 1905, Frye (W). Ferry Co. : Republic, 1912, Foster 2290 (F). Whitman Co.: Pullman, 1913, Weaver 25 (F). San Juan Co.: Mt. Constitution, $2200 \mathrm{ft}$., 1904, Frye 33 (W).

A common species, wherever rocks are plentiful.

15. Parmelia conspersa var. isidiata (Anzi) Berry. Monograph Gen. Parmelia in N. Am. north of Mexico. Ann. Mo. Bot. Gard. 28: 76. 1941.

Parmelia conspersa f. isidiata Anzi Cat. Lich. Sondr. 28. 1860. 
Thallus thickly beset with coralloid branchlets, otherwise like the species. Upper cortex reddish brown with paraphenylenediamine.

On rock: Yakima Co.: cliff near Naches, Ranger Station, 2400 ft., 1940, 3325; gulch near Naches, 1550 ft., 1940, 3180. Chelan Co.: butte near Chelan, 2000 ft., 1931, 1326. Columbia Co.: Dayton, 1700 ft., 1940, 2731. Klickitat Co.: Goldendale, $1600 \mathrm{ft}$., Foster 1165 (F), this specimen determined as Parmelia conspersa by G. K. Merrill.

At the present time this species has not been collected west of the summit of the Cascade Mountains.

16. Parmelia multispora Schneid., Guide to the Study of Lichens, 154. 1898.

Parmelia olivacea var. multispora Merrill. Bryol. 12 :73. 1909.

Thallus various shades of brown, small, membranous, smooth or rough and warty, short, wide, marginal lobes with rounded tips, edges elevated, coarsely crenate; below dark brown or black with short dark colored rhizoids in the center, at the margins light brown and devoid of rhizoids. Apothecia abundant, 1-4 mm. in diameter, sessile; disk brown, concave to flat; thalline margin entire or crenulate; asci 16-many-spored, Schneider (1898) reports possibly 100 spores in an ascus, ascus inflated-clavate; spores hyaline, subglobose to ovoid, nonseptate, 7.5-10.6 x 5.0-9.9 $\mu$.

On bark of various deciduous trees : Kittitas Co. : Roslyn, $2800 \mathrm{ft} ., 1931$, 898. Klickitat Co.: Goldendale, 2000 ft., 1931, 1682. Chelan Co.: Wenatchee Lake, 1800 ft., 1931, 1066; Rainbow Trail, 3600 ft., 1931, 1269. Lincoln Co. : Davenport, 2450 ft., 1931, 1595. Whitman Co. : Pullman, 2550 ft., 1931, 1576. Wahkiakum Co.: Cathlamet, 1907, Foster (F). Klickitat Co.: Goldendale, 1909, Foster 1163 (W). Ferry Co. : Republic, 1912, Foster 2291 (W). Spokane Co.: Spokane, 1907, T. A. Bonser (F).

A common species, rather widely distributed, especially on the eastern side of the Cascades. It is easily recognized by the numerous apothecia and the many-spored asci.

17. Parmelia rudecta Ach. Syn. Meth. Lich. 197. 1814.

Parmelia borreri var. rudecta Tuck. Enum. N. Amer. Lich. 49. 1845.

Thallus ochraceous-tawny (R), olive-brown (R), or grayish-olive $(R)$, coriaceous-membranous, wrinkled, or slightly rimose-reticulate, much divided, lobes rounded at the tips, somewhat imbricated, margins crenate, sparingly sorediate, abundantly isidiose, especially towards the center, with some coralloid branches; below white to pale brown or darker. Apothecia 3-6 $\mathrm{mm}$. in diameter, dark chestnut brown, sessile, deeply concave; thalline margin wavy, crenate, or irregular; asci 8-spored; spores hyaline, roundedellipsoid, nonseptate, $11.0-18.0 \times 7.0-10.0 \mu$. Medulla shows no color change with paraphenylenediamine.

On trees, decaying branches and logs, rarely on rock: Jefferson Co.: Olympic Mountains, 1915, J. M. Grant (F). 
18. Parmelia saxatilis (L.) Ach. Meth. Lich. 204. 1803.

Lichen saxatilis Linn. Sp. Pl. 1142. 1853.

Thallus ivory yellow (R), deep olive-buff $(R)$, tawny-olive $(R)$, cream color $(R)$, or rarely brown, cartilagineous-membranaceous, reticulate-rimose, lacunose, lobes narrow, sinuose, often pinnately many-cleft, tips retuse, crenate or truncate, often brownish, slightly isidiose; below black, tips brownish, with abundant, black rhizoids. Apothecia rare, 1-6 $\mathrm{mm}$. in diameter, sessile; disk chestnut-brown, concave; thalline margin entire, subcrenate or irregular; asci 8-spored; spores hyaline, ellipsoid, nonseptate, $14.0-19.8 \times 8.0-10.5 \mu$.

On bark, wood or rock, often on moss over rock: San Juan Co.: San Juan Island, Pt. Caution, 1940, 3279. Pacific Co.: Loomis Lake, 1931, 546. Kitsap Co.: Hidden Ranch, near Bremerton, 213 ft., 1930, 768. Thurston Co. : Grand Mound, 1940, 2979. Chelan Co. : Merritt, 2186 ft., 1931, $1087 a$. Kittitas Co.: South Cle Elum, 3000 ft., 1931, 876a. Klickitat Co.: Goldendale, 2000 ft., 1931, 1691. Ferry Co. : Laurier, 1644 ft., 1931, 1418. Clallam Co.: Olympic Hot Springs, 2000 ft., 1914, Foster 2866 (W). Grays Harbor Co.: Montesano, 1919, J. M. Grant 3006 (F). San Juan Co.: Orcas Island, 1904, Frye (F). Spokane Co.: Spokane, 1908, T. A. Bonser 53 (W).

A common species widely distributed. It is easily recognized by the reticulate-rimose thallus, the lack of soredia separates it from Parmelia sulcata.

19. Parmelia saxatilis var. Aizoni Del. in Duby, Bot. Gallic 2:602. 1830.

Thallus densely covered with grayish-brown isidia, so dense at times as to entirely replace the thallus, otherwise like the species; apothecia rare. Medulla reddish orange with paraphenylenediamine.

On bark, soil, and rock: San Juan Co. : San Juan Island, Friday Harbor, 1940, 3242; Mt. Constitution, Orcas Island, 1928, 2109. Pacific Co.: Long Beach, 1940, 2759. King Co.: Edgewood, 300 ft., 1940, 3086. Chelan Co.: Rainbow Trail, 3600 ft., 1931, 1270. Klickitat Co.: Goldendale, 2000 ft., 1931, 1697. Ferry Co.: Laurier, 1644 ft., 1931, 1416. Grays Harbor Co.: Aberdeen, Foster 922 (F); Montesano, 1919, J. M. Grant 3014 (F). Clallam Co.: Sequim, 1916, J. M. Grant 847 (F). Spokane Co.: Spokane, 1906, T. A. Bonser (F).

Rather common and widely distributed, similar in appearance to Parmelia rudecta, from which it can easily be separated by the red or reddish orange coloration of the medulla, when treated with paraphenylenediamine, the medulla of Parmelia rudecta shows no color change with paraphenylenediamine.

20. Parmelia sulcata Taylor in Mack., Fl. Hibern. 2:145. 1836.

Parmelia saxatilis var. sulcata (Taylor) Linds. Trans. Roy. Soc. of Edinburgh, $22: 228$. 1859. 
Thallus ivory yellow (R), deep olive-buff $(R)$, or rarely tawny-olive $(R)$, cartilaginous-membranaceous, reticulate-rimose, lacunose, thallus often cracked along the reticulations and on the margins, these cracks are usually filled with soredia, also scattered rounded or oblong masses of soredia, lobes rather broader than $P$. saxatilis, oblong to linear, pinnately branched, often imbricated, with wavy or roundly dentate margins, tips broad, never isidiose; below black, sometimes brown towards the margins, densely beset with stiff, black rhizoids. Apothecia rare, 1-6 mm. in diameter, sessile; disk chestnut brown, concave to deeply convex; thalline margin entire but often sorediate; asci 8-spored; spores hyaline, ellipsoid, nonseptate, $10.0-16.5 \times 6.6-8.2 \mu$.

On trees, wood, and rarely on rock: San Juan Co.: Brown Island, 1940, 3263; Orcas Island, Mt. Constitution, 2500 ft., 1928, 2052. Pacific Co.: Loomis Lake, 1931, 441. Kitsap Co.: Hidden Ranch, near Bremerton, 213 ft., 1930, 546a. Island Co.: Whidbey Island, 1931, 1772. Chelan Co.: Wenatchee Lake, $1800 \mathrm{ft} ., 1940,3166$. Island Co.: Whidbey Island, Langley, 1923, J. M. Grant (F). Clallam Co.: Sequim, 1916, J. M. Grant 815 (F). Snohomish Co.: Marysville, 1927, J. M. Grant (F). Chelan Co.: Blewett Pass, 4071 ft., 1929, J. M. Grant (F). Spokane Co.: Spokane, 1906, T. A. Bonser 8 (F).

Collected more frequently than $P$. saxatilis, which it resembles quite closely, but from which it can be easily distinguished by the abundant soredia. Plate VI, B.

21. Parmelia caperata (L.) Ach. Meth. Lich. 216. 1803.

Lichen caperatus Linn. Sp. P1. 1147. 1753.

Thallus old gold $(R)$, pale dull green-yellow $(R)$, or deep olive-buff $(\mathrm{R})$, coriaceous-membranaceous, forming an orbicular or irregular mat, closely attached to the substratum, rough and wrinkled, plicate, somewhat lacunose, sometimes abundantly sorediate, or rarely bearing coralloid branches, lobes short and broad, slightly imbricated, margins sinuose, often sorediate, tips rounded, entire or crenate; below purplish brown or black, with abundant rhizoids near the center, margins smooth and shiny, brown, without rhizoids. Apothecia rare, 1-8 mm. in diameter, sessile; disk chestnut-brown, concave to deeply concave; thalline margin entire, becoming crenulate and finally sorediate, or isidiose; asci 8-spored; spores hyaline, ellipsoid, nonseptate, $12.0-20.0 \times 7.5-9.0 \mu$. Thallus shows no color change with paraphenylenediamine.

On bark or wood, more rarely on rock or moss: Walla Walla Co.: Wallula, 200 ft., Foster 293 (F).

A rare lichen in Washington, but very common in other parts of the United States.

22. Parmelia crinita Ach. Syn. Meth. Lich. 196. 1814. 
Thallus gray green, deep olive-buff $(R)$, cream-buff $(R)$, or chamois (R), large, broad lobes, much divided, abundantly isidiose, tips rounded, margins elevated, bearing prominent black cilia; below black, with large concolorous rhizoids at the center, margins brown, without rhizoids. Apothecia not common, 3-12 mm. in diameter, sessile to subpedicellate, deeply concave, sometimes cup-shaped, chestnut-brown; thalline margin isidiose or granulose, sometimes ciliate; asci 8-spored; spores hyaline, ellipsoid, nonseptate, $23.0-32.0 \times 16.0-18.0 \mu$. Thallus shows no color change with paraphenylenediamine.

On trees and wood, more rarely on rocks: Skagit Co. : Anacortes, 1908, T. C. Frye (D). I have not seen this specimen.

This lichen can easily be distinguished by the broad mat and the black cilia on the margins.

23. Parmelia perlata (Huds.) Ach. Meth. Lich. 216. 1803.

Lichen perlatus Huds. Fl. Angl. 448. 1762.

Thallus grayish white, deep olive-buff ( $R$ ), tawny-olive $(R)$, creamcolor ( $R$ ), membranaceous, smooth, wide spreading, lobes broad, not much elongated, branched, imbricated, margins ascending, undulate, bearing lobules, which are often digitately clustered, and abundantly covered with soredia, margins sometimes slightly ciliate; below black with brown margins, smooth, or wrinkled in places, with scattered black rhizoids. Apothecia not common, $2-12 \mathrm{~mm}$. in diameter, sessile or short pedicellate; disk chestnut brown $(\mathrm{R})$ or bay $(\mathrm{R})$; thalline margin entire; asci 8-spored; spores hyaline, ellipsoid, nonseptate, 11.0-17.0 x 7.0-11.0 $\mu$.

On rock or trees: Pacific Co.: Chinook, 1931, 793. Kitsap Co. : Hidden Ranch near Bremerton, 213 ft., 1930, 1888a. Clallam Co.: Cape Flattery, A. H. Smith, 1891 (M). San Juan Co.: Orcas Island, Olga, 1906, Fink 344 (F). Plate V, D, and Plate VI, A.

The densely sorediate margins are a distinguishing characteristic.

24. Parmelia perlata var. ciliata (DC.) Duby, Bot. Gall. 2:601. 1830.

Lobaria perlata var. ciliata DC. in Lam. \& DC. F. Fr. 3rd ed., 2:403. 1805.

Thallus frequently besprinkled with isidia, margins bearing numerous black cilia; below rhizoids rather scarce, ohterwise like the species, sterile in Washington.

On rocks and trees: Pacific Co.: Long Beach, 1928, 1912; Nahcotta, 1931, 470. Chinook, 1940, 2803. San Juan Co. : Friday Harbor, 1931, 2055. Grays Harbor Co.: Westport, 1919, J. M. Grant 3019.

\section{Cetraria Ach. Meth. Lich. 292. 1803.}

Thallus foliose, subfruticose, or fruticose, smooth, sorediate, or isidiose; foliose forms more or less ascendant, lobed; fruticose forms erect, tufted, branched, at times channeled; well-developed upper and lower plectenchy- 
matous cortices, medullary, and algal layers, medulla cottony; lower surface either lighter or darker than the upper, rhizoids scarce. Apothecia terminal or marginal, circular or irregular in shape, sessile or subpedicellate; thalline margin present; hypothecium hyaline or pale brown; paraphyses unbranched or rarely branched, conglutinate and septate; asci 6-8-spored, hyaline, nonseptate. Spermatia lateral.

A. Thallus foliose.

B. Bright yellow.

C. Apothecia usually abundant, margins of thallus never sorediate.

C. Apothecia very rare, margins of thallus sorediate...........7. C. pinastri B. Not yellow.

D. Some shade of brown.

E. Margins bearing prominent white soredia; apothecia rare.

8. C. scutata

E. Margins bearing dark papillae; apothecia common.

D. Greenish gray or straw color.

1. C. fahlunensis

F. Definitely and deeply reticulate-lacunose, thallus with IKI gives no color change.

G. Lobes narrow, elongated, often pendulous......6. C. stenophylla

G. Lobes broad and widely spreading...................... . C. lacunosa

F. Not definitely and deeply reticulate-lacunose, thallus with IKI, blue.

H. Lobes narrow, elongated, margins sorediate, often with short, jagged coralloid branchlets.

3. C. Tuckermani

H. Lobes broader, short, margins sorediate, isidiose, or with coralloid branchlets.

2. C. glauca

A. Thallus fruticose to subfruticose.

I. Yellow, green yellow, or cinnamon buff, margins never ciliose-spinulose. J. Lobes long and narrow, rarely branched above, margins often joining to form a tube.

9. C. cucullata

J. Lobes wider, much branched above, deeply laciniate.....14. C. nivalis

I. Some shade of brown or green, margins always somewhat ciliosespinulose.

$\mathrm{K}$. Thallus blue with IKI, margins abundantly ciliose-spinulose.

11. C. islandica

$\mathrm{K}$. Thallus not changing color with IKI, margins sparingly ciliosespinulose.

L. Always growing on trees or shrubs.

L. Never growing on trees or shrubs.

12. C. arborialis

10. C. hiascens

I. Black or very dark brown, margins never ciliose-spinulose.

13. C. Merrillii

1. Cetraria fahlunensis (L.) Schaer., Lich. Helvet. Spicil. sect. 4-5, 255. 1833.

Lichen fahlunensis Linn. Sp. P1. 1143. 1753. 
Thallus dark brown or chestnut-brown ( $R$ ), foliose, suborbicular, appressed, imbricated, lobes laciniate, somewhat ascending, smooth or slightly channeled, segments often much entangled, margins crenate, crisped, sometimes with dark colored papillae; below various shades of brown or black, with a few scattered rhizoids. Apothecia numerous, often entirely covering the lobes, marginal, 1-9 mm. in diameter, adnate; disk chestnut brown, concave to flat, sometimes irregular and dilated; thalline margin entire or rugose, crenulate; spores hyaline, ellipsoid, nonseptate, 5.0-10.8 x 3.3-6.0 $\mu$.

On rocks: King Co.: Snow Lake Trail, 3500 ft., 1931, 831. Pierce Co.: White River Camp, 3900 ft., 1928, 1897; Mt. Rainier, near Paradise River, 1909, Foster 1062 (W).

Dark marginal papillae help to identify this species.

2. Cetraria glauca (L.) Ach. Meth. Lich. 296. 1803.

Lichen glaucus Linn. Sp. P1. 1148. 1753.

Thallus sea-foam yellow (R), pale grass green $(R)$, pale glaucous green $(R)$, ivory yellow $(R)$, olive-buff $(R)$, or gray, foliose, membranaceous, smooth or slightly lacunose, occasionally sprinkled with grayish white soredia or isidia, lobes narrow or broad, entire, crenate, sinuate or lacerate, margins ascendant, often thickened, crenate, jagged, often sorediate, more rarely isidiose; below white, brown, or black, often black with brown margins, or brown with white margins, dull or shiny, smooth or lacunose, rhizoids rare, if present very small and scattered. Apothecia rare, marginal or submarginal, 2-6 mm. in diameter, adnate; disk chestnut, brown, flat, wavy, thalline margin irregular and disappearing; spores hyaline, ellipsoid, 4.6-6.0 x 3.0-4.0 $\mu$. Thallus blue with IKI.

On trees or fallen branches, rarely on rock: Pacific Co.: North Head, $211 \mathrm{ft} ., 1930,457 a$. Grays Harbor Co. : Lake Quinault, $300 \mathrm{ft.,} \mathrm{1931,} 1210$. San Juan Co.: Friday Harbor, 1928, 2057. Thurston Co.: Gate, 1940, 2967. Kitsap Co.: Hidden Ranch, near Bremerton, 213 ft., 521. Pierce Co.: Silver Springs, $2200 \mathrm{ft}$., 1931, 1127. King-Kittitas Co.: Snoqualmie Pass, 3010 ft., 1931, 813. Chelan Co.: White Rock Camp, $2200 \mathrm{ft} ., 1931,1045$. Stevens Co.: Boyd's, 1470 ft., 1439. Whitman Co.: Tekoa Mountains, 2900 ft., 1597. Columbia Co.: Blue Mountains, $3500 \mathrm{ft} ., 1931$, 1631. San Juan Co.: San Juan Island, 1906, Fink (F). Snohomish Co.: Marysville, 1926, J. M. Grant (F). Pierce Co. : Paradise Park, 1909, Foster (F).

One of the most common and widely distributed lichens in the state. Plate VI, C, D. Plate VII, A. B.

3. Cetraria Tuckermani Herre, Proc. Wash. Acad. Sci. $7: 340.1906$.

Cetraria glauca var. stenophylla Tuck. Syn. N. Am. Lich. 1:35. 1882.

Thallus olive-buff $(\mathrm{R})$, greenish gray, or pale straw color, foliose, cartilaginous-coriaceous, lobes narrow elongated, lax, channeled, smooth or slightly pitted, occasionally sorediate or isidiose, margins ascending, rarely entire, crenate to irregularly jagged and torn, often sorediate or with short coralloid branchlets; below usually black, sometimes only black near the 
base, becoming white or brown near the tips, sometimes shallow pitted. Apothecia very rare. Thallus blue with IKI.

On trees: Pacific Co.: Chinook, 1940, 2820. Clallam Co.: Lake Sutherland, 590 ft., 1931, 1031. San Juan Co.: Brown Island, 1940, 3201. Kitsap Co.: Hidden Ranch near Bremerton, 213 ft., 1940, 3294. Pierce Co. : Silver Springs, 2670 ft., 1931, 719; Mt. Rainier, trail to Seattle Park, 4000 ft., 1940, 3058. Whatcom Co.: Ruth Mountain, 5500 ft., 1928, L. Sunquist 1895 (W). Grays Harbor Co.: Westport, 1919, J. M. Grant (F). Clallam Co.: Sequim, 1915, J. M. Grant (F). San Juan Co.: Friday Harbor, 1906, Fink 84 (W). Olympic Mountains, Camp Elkhorn, 1800 ft., 1907, Frye 60 (W). Pierce Co.: Mt. Rainier, 1906, Foster (F).

A very common species west of the Cascades. A bark lichen easily identified by the long, lax lobes, with black under surface, very rarely fruiting.

4. Cetraria juniperina (L.) Ach. Meth. Lich. 299. 1803.

Lichen juniperinus Linn. Sp. P1. 1147. 1753.

Thallus bright yellow, straw color ( $R)$, lemon chrome $(R)$, yellowish or citrine $(R)$, membranaceous, expanded or tufted, lacunose, lobes narrow, small, irregularly branched, margins erect, often curled, crenate, erosecrenate, or dentate; below paler or the same color. Apothecia numerous, submarginal, $2-12 \mathrm{~mm}$. in diameter, sessile or subpedicellate; disk chestnutbrown $(R)$, concave to convex; thalline margin crenulate or tuberculate, the same color as the thallus; spores hyaline, ellipsoid, nonseptate, 4.0-9.9 $\times 3.0-5.0 \mu$.

On trees and fallen branches, rarely on rock or soil: San Juan Co.: Friday Harbor, 1928, 2061. Mason Co.: Shelton, 1931, 1221. Kittitas Co.: Easton, 2168 ft., 1931, 835. Yakima Co.: American River Camp, 2800 ft., 1931, 956. Chelan Co.: Stehekin, 1140 ft., 1931, 1284a. Ferry Co.: Mt. Gibraltar, near Republic, 3100 ft., 1940, 3149. Whitman Co.: Tekoa Mountains, 2900 ft., 1931, 1605a. Cascade Mountains, Brandegee (F). Klickitat Co.: Goldendale, 1909, Foster 1161 (W). Mason Co.: 1890, C. V. Piper 13 (W). San Juan Co.: Friday Harbor, 1906, Fink (W).

The bright yellow thallus with white medulla and the brown disk of the apothecium make this a conspicuous lichen.

5. Cetraria lacunosa Ach. Meth. Lich. 295. 1803.

Thallus olive-buff (R), Isabella color (R), greenish-gray, or straw color, cartilaginous-coriaceous, deeply reticulate-lacunose, ridges sometimes isidiose, lobes broad and widely spreading, crowded, irregularly branched, margins ascending, crenate-incised, or sometimes entire, tips rounded; below sometimes almost white, brown, or black. Apothecia numerous, marginal or submarginal, $1-12 \mathrm{~mm}$. in diameter, sessile or subpedicellate; disk chestnut-brown, concave, thalline margin entire; spores hyaline, ellipsoid, nonseptate, $6.6-8.0 \times 3.3-5.0 \mu$. Thallus shows no color change with IKI. 
On trees: Pacific Co.: Loomis Lake, 1931, 438. Grays Harbor Co.: Lake Quinault, 1931, 300 ft., 1212. Wahkiakum Co.: Cathlamet, 1940, 2916. Clallam Co.: Elwha River Trail, 1500 ft., 1940, 2931. Kitsap Co.: Hidden Ranch, near Bremerton, 213 ft., 1940, 3284. Pierce Co.: Mt. Rainier, trail to Seattle Park, 4000 ft., 1940, 3050. Wahkiakum Co.: Cathlamet, 1907, Foster 501 (F). Grays Harbor Co., Foster 912 (F).

The deeply lacunose-reticulate nature of the thallus makes this an easy lichen to recognize.

6. Cetraria stenophylla (Tuck.) Merrill, Bryol. $13: 27.1913$.

Cetraria lacunose var. stenophylla Tuck. Syn. N. Am. Lich. 1 :35. 1882.

Thallus olive-buff $(R)$, straw yellow $(R)$, or ivory yellow $(R)$, lobes narrow, elongated, cartilaginous-coriaceous, stiff and rigid, channeled, often crowded, margins ascending, often entire, torn, or torn-crenate, sometimes black, tuberculate; below usually white, sometimes brown or black. Apothecia chestnut-brown ( $R$ ), concave, surface undulate; thalline margin entire, thin, sometimes disappearing; spores hyaline, subglobose or ellipsoid, nonseptate, 4.9-8.0 $\times 3.3-5.0 \mu$. Thallus shows no color change with IKI.

On trees: San Juan Co.: Orcas Island, Mt. Constitution, 2200 ft., 1906, fruiting, Fink (F) ; 1904, Frye (F) ; Mt. Constitution, 1907, Foster $564(\mathrm{~F})$.

This species can be separated easily from $C$. Tuckermani by the reticulate-lacunose thallus, the entire margin, and the frequent apothecia. It occurs much more rarely than $C$. Tuckermani in the state.

7. Cetraria pinastri (Scop.) Röhl. Deutschl. Fl. 3, Abt. 2, 113. 1813.

Lichen pinastri Scop. F1. Carniol. 2nd ed., 2:382. 1772.

Cetraria juniperina var. pinastri (Scop.) Ach.

Thallus some shade of yellow, lemon yellow (R), lemon chrome (R), or antimony yellow (R), membranaceous, slightly lacunose, lobes flattened, roundish, sometimes imbricated, margins often erect, crenate, and abundantly bright yellow, sorediate; below usually the same color, sometimes slightly darker, rarely ever found fruiting, sterile in this state.

On trees: Yakima Co.: Lodge Pole Pine Camp, 3500 ft., 1940, 3311.

So far as the writer is able to find out, this is the only collection that has ever been made of this species in the state. It is easily recognized by the bright yellow color and the sorediate margins.

8. Cetraria scutata (Wulf.) Poetsch, in Poetsch and Schiderm. Syst. Aufzahl. samenlos. P1. 262. 1872.

Lichen scutatus Wulf., in Jacq. Coll. Bot. 4 :268. 1790.

Cetraria saepincola var. chlorophylla Schaer.

Cetraria chlorophylla (Willd.) Wainio.

Thallus olive green, olive-brown ( $R$ ), or snuff brown (R), lobes numerous, crowded, short, prostrate or ascending, smooth or slightly rough, 
with occasional, small, scattered patches of white soredia, margins crenate, sinuate, or lacerate, usually covered with prominent white soredia; below paler, wrinkled, with scattered rhizoids. Apothecia rare, marginal or submarginal, small, $0.5-1.5 \mathrm{~mm}$. in diameter, adnate to sessile; disk chestnutbrown $(R)$, concave to convex; thalline margin entire or crenulate; spores hyaline, ellipsoid, nonseptate, 7.0-9.0 x 3.5-6.0 $\mu$.

On trees, fallen limbs, and fences; more rarely rock or in a thin layer of soil over rock: Pacific Co.: Long Beach, 1940, 2936. Snohomish Co.: Chase Bog, 1940, 3341. King Co.: Edgewood, 300 ft., 1940, 3083a. Thurston Co.: Grand Mound, 1940, 2977. Kittitas Co.: Easton, 2168 ft., 1931, 848. Yakima Co.: Bumping Lake, $3400 \mathrm{ft} ., 1931$, 930. Chelan Co.: Rainbow Trail, 3800 ft., 1931, 1280. Ferry Co.: Mt. Gibraltar, near Republic, 3100 ft., 1940, 3156. Grays Harbor Co.: Westport, 1908, Foster 663 (F). Clallam Co.: Dungeness, 1913, Foster 2549 (F). Klickitat Co.: Goldendale, 1909, Foster 1160 (W). San Juan Co.: Friday Harbor, 1906, Fink 77 (W). Herre (1917) reports this species from Whatcom Co., "on twigs and old fences in the lowlands."

A common bark lichen, rather widely distributed. It is usually some shade of brown, with conspicuous white soredia on the margins.

9. Cetraria cucullata (Bellard.) Ach. Meth. Lich. 293. 1803.

Lichen cucullatus Bellard. Observo. Bot. 54. 1788.

Thallus pale yellow, cream-buff ( $R$ ), cinnamon-buff ( $R$ ), or dark olivebuff $(R)$, at times reddish purple towards the base, fruticose, erect, tufted, smooth or slightly lacunose, dull, margins undulate, dentate, or crenate, often meeting to form a tube, more rarely flattened. Apothecia $5-10 \mathrm{~mm}$. in diameter, usually terminal, adnate to the lower side of the lobe; disk reddish brown, often much dilated; thalline margin thin, entire or undulate; spores hyaline, ellipsoid, nonseptate, 8.0-9.0 × 3.0-4.0 $\mu$.

On soil: Olympic Mountains, $6000 \mathrm{ft} ., 1915, J$. M. Grant (F).

A rare species, found only in the high mountain meadows.

10. Cetraria hiascens (Fries) Th. Fr. Lich. Scand. 1 :98. 1871-74.

Cetraria aculeata var. hiascens Fries, Lich. Europ. Reform. 36. 1831.

Cetraria islandica var. Delisei Bory.

Thallus reddish brown, sepia ( $R$ ), tawny-olive (R), Isabella color $(R)$, or chamois $(R)$, many lobed, flat or partly connivent, lacunose, much branched toward the tips, margins dentate, crenulate, or sparingly spinulose toward the tips, entire near the base; below paler and frequently bearing slightly sunken, round sorediate areas. Apothecia terminal or marginal, 3-6 $\mathrm{mm}$. in diameter, adnate to the upper surface of the lobes; disk pale or dark chestnut-brown $(R)$, concave to flat; thalline margin entire or dentate; spores hyaline, ellipsoid, nonseptate, 4.0-8.0 $\times$ 2.0-6.0 $\mu$. Thallus unchanged in color with IKI. 
On soil: Ferry Co.: Republic, 2650 ft., 1912, Foster 2283 (F).

Rare, usually in the mountains. It can easily be distinguished from C. islandica.

11. Cetraria islandica (L.) Ach. Meth. Lich. 293. 1803.

Lichen islandicus Linn. Sp. P1. 1145. 1753.

Thallus various shades of brown, light brownish-olive $(R)$, or sepia $(R)$, sometimes reddish towards the base, foliose or fruticose, tufted, erect, rigid, stiff, lobes narrow, branched dichotomously or irregularly, smooth, shiny, sometimes lacunose or channeled, flat or often rolled into a tube, margins involute, abundantly ciliate-spinulose from the tips to the base; below darker or sometimes lighter, with sunken, round, sorediate areas usually abundant. Apothecia usually terminal, 1-10 mm. in diameter, adnate to the upper surface of the lobe; disk chestnut-brown ( $R$ ) or darker, concave to convex, surface often uneven; thalline margin thin, entire or crenulate, soon disappearing; spores hyaline, ellipsoid, nonseptate, 6.6-9.9 × 3.3-5.0 $\mu$. Thallus blue with IKI.

On soil: Whatcom Co.: Ruth Mountain, 5500 ft., 1928, L. Sunquist 1890 (W). Herre (1917) reports it from Whatcom Co. : Mt. Baker.

An alpine species that can be distinguished easily by the abundant ciliate-spinulose margins and its habitat, always grows on soil.

12. Cetraria arborialis (Merrill) Howard comb. nov.

Cetraria islandica mod. arborialis Merrill, Bryol. 9:4. 1906.

Thallus various shades of brown, sepia (R), light brownish olive ( $R$ ), light grape green $(R)$, grape green $(R)$, or yellowish glaucous $(R)$, foliose or fruticose, cartilagenous, lobes elongated, channeled or almost flat, smooth, dull or shiny, irregularly dichotomously branched, often forming matted masses, margins entire, undulate or crenate, here and there ciliate-spinulose, tips very rarely spinulose; below paler, slightly lacunose. Apothecia usually terminal, 3-6 mm. in diameter, adnate to upper surface; disk pale brown, concave to convex, surface becoming uneven; thalline margin thin and slightly crenulate; spores hyaline, globose, 3-4 $\mu$ in diameter. Thallus shows no color change with IKI.

On shrubs, living or dead, usually a species of Vaccinium: Pierce Co.: Mt. Rainier, Berkeley Park, 6000 ft., 1931, 610; Mowich Lake Trail, 3500 ft., 1940, 3062; trail to Seattle Park, $4000 \mathrm{ft} ., 1940$, 3059. Yakima Co.: Dewey Lake, $4700 \mathrm{ft}$., 1940, 3354. Whatcom Co. : Ruth Mountain, $5500 \mathrm{ft}$., 1928, L. Sunquist 1890a (W). Olympic Mountains on low shrubs, $5000 \mathrm{ft}$., 1895, C. V. Piper 528 (C), this specimen determined as Cetraria islandica mod. arborialis Merrill, Fide R. H. Howe, Jr. Jefferson Co.: Olympic Mountains, Queets River Valley, $5000 \mathrm{ft} ., 1907$, Frye 14 (W), this specimen determined as Cetraria islandica var. arboricoline Tuck. by G. K. Merrill. Clallam Co.: Olympic Hot Springs on Vaccinium sp., 1914, Foster (W), this specimen determined as Cetraria islandica.

An alpine species, easily separated from $C$. islandica by the difference in 
habitat. $C$. arborialis grows on wood, $C$. islandica on soil; also by the lack of color change of the thallus with IKI.

13. Cetraria Merrillii DR. Arkiv för Bot. 20, A, No. $11: 42.1926$.

Cetraria californica Merrill, Bryol., $13: 28.1910$. (Not Tuck.)

Thallus greenish-black, fuscous-black (R), or black, foliose or subfruticose, ascending or erect, caepsitose, lobes rather narrow, compressed, lacunose, margins deeply cut into fingerlike lobules, tips acute; below paler. Apothecia abundant, marginal or submarginal, 1-6 mm. in diameter, sessile ; disk dark brown or black, concave to flat; thalline margin crenulate or dentate; spores hyaline, ellipsoid, nonseptate, 5.0-8.5 $\times 3.0-4.0 \mu$.

On twigs: Yakima Co.: Deadhorse Hill, 4000 ft., 1931, 961; Lodge Pole Pine Camp, 3500 ft., 1940, 3303; Rattlesnake Canyon, 2000 ft., 1931, 1544. Ferry Co. : Mt. Gibraltar, 3100 ft., 1940, 3150.

A distinctive Cetraria, although rather small.

14. Cetraria nivalis (L.) Ach. Meth. Lich. 294. 1803.

Lichen nivalis Linn. Sp. P1. 1145. 1753.

Thallus pale yellow, maize yellow (R), cream color (R), Naples yellow $(R)$, mustard yellow (R), or bright yellow, fruticose or subfruticose, reticulate-lacunose, often channeled, or smooth, usually dull, much divided, lobes deeply laciniate, margins irregular, dentate with truncate points; below slightly darker and duller. Apothecia rather rare, usually terminal, 2-12 $\mathrm{mm}$. in diameter, adnate to the under surface of the lobe; disk fawn color $(\mathrm{R})$ or brown to pale brownish pink, flat becoming uneven; thalline margin crenulate; spores hyaline, ellipsoid, 7.0-10.0 x 3.0-5.0 $\mu$.

On the earth in alpine regions: Jefferson Co.: Olympic Mountains, $6000 \mathrm{ft} ., 1918, J . M$. Grant (F).

This beautiful, yellow, somewhat leaf-like lichen is easily recognized. Rather strange that it has not been collected in other mountainous regions of the state.

56. Nephromopsis Müll. Arg. Flora $74: 374.1891$.

Thallus much like Cetraria but distinguished from it by the apothecia, which originate on the lower side, but by the twisting and turning of the lobes are brought on to the upper surface, prominent tubercules scattered on the lobes or confined to the margins, or in both places; below smooth, lacunose, or wrinkled, rhizoids scarce or more abundant. Apothecia medium sized, round, adnate or sessile, terminal or marginal; disk some shade of brown; asci 8-spored; spores hyaline, nonseptate.

A. Thallus some shade of yellow.

2. N. pallidula

A. Thallus not yellow.

B. Lobes narrow, much branched, olive brown or brown.......1. N. ciliaris B. Lobes broad, not much branched, dark brown............. 3. $N$. platyphylla 
1. Nephromopsis ciliaris (Ach.) Hue, Nouv. Archiv. du Museum ser. 4, $1: 216.1899$.

Cetraria ciliaris Ach. Lich. Univers. 508. 1810.

Thallus buffy brown (R), mummy brown (R), grayish olive (R), or light brownish olive, cartilageneous-membranaceous, foliose, irregularly pitted, often smooth and shiny, with scattered black tubercles, much divided, lobes long and narrow, crowded, sinuate-laciniate, margins crenate, bearing minute black or dark brown tubercules, and occasionally scattered fibrils; below paler, lacunose, with a few scattered rhizoids, or numerous rhizoids. Apothecia marginal, 2-8 $\mathrm{mm}$. in diameter, sessile; disk hazel ( $R$ ), or chestnut-brown $(R)$, often shiny, concave to convex; thalline margin crenulate; spores hyaline, globose to subglobose, nonseptate, $4.0-7.0 \mu$ in diameter.

On trees, fences, or soil: Pacific Co. : North Head, 1930, 457. San Juan County: San Juan Island, English Camp, 1928, 2064. Island Co. : Whidbey Island, 1931, 1774a. King Co.: Richmond Highlands, 300 ft., 572. Kittitas Co.: Lake Cle Elum, 2200 ft., 1931, 890b. Wahkiakum Co.: Cathlamet, 1907, Foster 509 (F). Island Co.: Whidbey Island, Langley, 1920, J. M. Grant 3025 (F). Clallam Co. : Dungeness, 1913, Foster 2549 (W). Washington Territory, Suksdorf 138 (T).

The prominent dark brown or black tubercules characterize this species.

2. Nephromopsis pallidula (Tuck.) Riddle, Bryol. 18:28. 1915.

Cetraria pallidula Tuck. in Riddle 1.s.c.

Thallus pale yellow or chartreuse yellow ( $R$ ), foliose, cartilaginous, rigid, lobes broad and rounded, irregularly rugose-wrinkled or somewhat lacunose-reticulate, margin ascendant, crenate, with marginal or submarginal black tubercles, medulla white; below concolorous or paler, with a few scattered rhizoids. Apothecia marginal, 3-5 mm. in diameter, sessile; disk hazel $(\mathrm{R})$, concave; thalline margin yellow, crenate, finally becoming tuberculate; spores hyaline, globose, nonseptate, 4.0-6.0 $\mu$ in diameter.

On bark: Kittitas Co.: Lake Cle Elum, 2200 ft., 1931, 890. Yakima Co.: Lodge Pole Pine Camp, 3500 ft., 1940, 3310 ; American River Camp, 2800 ft., 1931, 956. Washington Territory, Falcon Valley, 1881, C. G. Pringle (W). Klickitat Co.: Goldendale, 1909, Foster 1182 (F), this specimen determined as Cetraria corrugata Merrill Nov. sp.

The yellow thallus with white medulla, and black tubercles, make this a showy lichen.

3. Nephromopsis platyphylla (Tuck.) Herre, Proc. Wash. Acad. Sci. $12: 210.1910$.

Cetraria platyphylla Tuck. Syn. N. Am. Lich. $1: 34.1882$.

Thallus buffy-brown ( $R$ ) or dark brown, foliose, cartilagineous, rigid, rather thick, reticulate-lacunose, rough, tuberculate, not much divided, lobes broad, tips rounded, ascendant, margins crenate or jagged, tuberculate; 
below paler, wrinkled, rhizoids scarce, scattered, usually only at points of attachment to the substratum. Apothecia marginal, 1-7 mm. in diameter, adnate; disk hazel $(R)$ or chestnut-brown ( $R)$, concave; thalline margin tuberculate; spores hyaline, spherical to subspherical, 4.0-8.0 $\mu$ in diameter.

On trees or fallen branches : Pierce Co.: Mt. Rainier, Grand Park, 5575 ft., 656; trail to Moraine Park, $3300 \mathrm{ft} ., 3005$; Paradise Park, $5550 \mathrm{ft}$., 1931, 1893. Yakima Co.: Bumping Lake, 3400 ft., 1931, 938. Chelan Co.: Merritt, 2186 ft., 1080. Spokane Co.: Mt. Spokane, 5808 ft., 1931, 1489a. Columbia Co.: Blue Mountains, 3500 ft., 1931, 1621. King Co.: Summit, 1907, Foster 650 (F). San Juan Co.: Orcas Island, Mt. Constitution, 2200 ft., 1904, Frye (F). Washington Territory, Suksdorf (F). Ferry Co.: Republic, 1912, Foster 2285 (F). Clallam Co.: Mt. Angeles, 3000 ft., 1911, Foster 1786 (W) ; Olympic Mountains, Crystal Ridge, 1939, A. H. Smith $14981(\mathrm{~F})$.

The dark brown color, the thick tough texture of the thallus, and the short lobes distinguish this species from $N$. ciliaris. It has not been collected below $2000 \mathrm{ft}$. 


\section{USNEACEAE}

Thallus fruticose, erect, or pendulous, usually radial, sometimes dorsoventral, differentiated into a typical plectenchymatous cortex surrounding the algal layer and medulla, the medulla being cottony, cobweb-like, solid or hollow, attached to the substratum by a holdfast or scattered rhizoids. Apothecia circular, marginal, subterminal, or terminal, sessile or subpedicellate; proper margin absent, thalline margin present; asci 1-8-spored; spores usually hyaline, rarely brown, nonseptate, 1-3-septate or muriform. Alga Protococcus.

A. Medulla cottony.

B. Medulla solid cotton.

C. Thallus soft and flaccid, some shade of grayish-green....57. Everma C. Thallus stiff and rather wiry, chestnut brown to black.

60. Cornicularia

B. Medulla not solid cotton, thallus rather stiff, with a strong central medullary cord.

62. Usnea

A. Medulla stiff or cobweb-like.

D. Apothecia unknown, podetia hollow, always light colored, usually white.

63. Thamnolia

D. Apothecia rather common, podetia not present.

E. Several definite medullary cords.

58. Letharia

E. No definite medullary cord.

F. Thallus filamentous, wiry or hair-like, center often hollow, spores nonseptate. …...............................................59. Alectoria

F. Thallus narrowly membranaceous, cartilaginous, stiff, often with a flattened appearance, especially where it is attached to the substratum, spores 1-3-septate.

61. Ramalina

57. Evernia Ach. Lichenogr. Univers. 84. 1810.

Thallus gray or greenish gray, fruticose, tufted, or shrubby, erect or prostrate, dorsi-ventral, flaccid or somewhat stiff, much branched, often matted and twisted, with a thin, irregular, plectenchymatous cortex, entirely surrounding the algal layer and cottony medulla, composed of loosely interwoven hyphae, attached to the substratum by a holdfast. Apothecia medium to large, circular, terminal or marginal, sessile or subpedicellate, 
thalline margin present; hypothecium hyaline; paraphyses thick, unbranched; asci 8-spored; spores hyaline, nonseptate.

A. Soft and flaccid, lobes flattened, angular.

B. Lobes slightly pitted, often channeled near base, mealy sorediate.

1. E. prunastri

B. Lobes deeply lacunose, densely beset with round to flat masses of soredia.

2. E. prunastri var. sorediifera

A. Rather stiff and rigid, lobes terete to subterete, angular.

3. E. thamnodes

1. Evernia prunastri (L.) Ach. Lichenogr. Univers. 442. 1810.

Lichen prunastri Linn. Sp. P1. 1147. 1753.

Thallus tufted, olive-buff ( $R$ ), grayish-olive ( $R$ ), or Isabella color ( $R$ ), erect or pendulous, very much branched or irregularly dichotomously, branches flattened, angular, pitted, often bearing mealy confluent soredia, often channeled near the base, tips forked, specimens with wide branches paler below. Apothecia rare, marginal, 2-6 mm. in diameter, subpedicellate; disk dark chestnut-brown ( $R$ ), concave; thalline margin inflexed; spores hyaline, short ellipsoid, nonseptate, $5.0-8.0 \times 3.3-6.0 \mu$.

On trees, shrubs, fallen branches, fences, and more rarely on rock: Clallam Co.: Lake Sutherland, 590 ft., 1931, 1116; Cedarville, 300 ft., 1940, 2889. San Juan Co.: Shaw Island, 1928, 2088. Wahkiakum Co.: Cathlamet, 1940, 2860. Thurston Co.: Grand Mound, 1940, 2976. Kitsap Co.: Hidden Ranch near Bremerton, 213 ft., 1940, 3279. King Co. : Edgewood, 300 ft., 1940, 3082. Klickitat Co.: Goldendale, 2000 ft., 1931, 1700. Whitman Co.: Tekoa Mountains, 1931, 2900 ft., 1606. Grays Harbor Co.: Montesano, 1919, J. M. Grant 3022 (F). Snohomish Co.: Marysville, 1929, J. M. Grant (F). Spokane Co. : Biglowe Gulch, near Spokane, 1905, T. A. Bonser (W).

This lichen is easily recognized by the cottony medulla. It grows abundantly in central Europe on oak trees, where it is called oak moss, and is used as a base in the manufacture of perfumes. Grows on oaks in certain regions of the state. Plate VII, C.

2. Evernia prunastri var. sorediifera Ach. Lichenogr. Univers. 443. 1810.

Thallus thicker, lobes shorter and broader, deeply lacunose and densely beset with round to flat masses of soredia; otherwise like the species. Sterile.

On trees and fallen branches: San Juan Co.: San Juan Island, 1930, 504. Yakima Co.: Satus Road, 1054 ft., 1673. Clallam Co.: Dungeness, 1913, Foster 2533 (W).

3. Evernia thamnodes (Flw.) Arn. Verhandl. Zool.-Bot. Gesellsch. Wien. $23: 110.1873$.

Evernia prunastri var. thamnodes Flw. Die merk. und selten. Flecht. Hirschberg. Warmbrunn 5. 1839. 
Thallus grayish-olive ( $R$ ), light brownish olive ( $R$ ), or pale yellow, shrubby, stiff, erect or prostrate, lobes subterete, angular, rarely flattened, long pitted, with a somewhat cracked cortex, rough and scurfy, tips of branches acuminate, soredia abundant, sterile in the state.

On trees and fences: Klickitat Co.: Goldendale, 2000 ft., 1931, 1702.

58. Letharia (Th. Fr.) Zahlbr. Hedwigia 31 :34. 1892.

Thallus yellow or yellowish green, fruticose, erect or pendulous, much branched, branches subterete, with a thin irregular plectenchymatous cortex surrounding the algal layer and the medulla, medulla cobweb-like with strong medullary cords running through it, attached to the substratum by a holdfast. Apothecia circular large to very large, subterminal or terminal, pedicellate; thalline margin prominent; hypothecium hyaline; paraphyses stout, unbranched; asci 8-spored; spores hyaline, nonseptate.

1. Letharia vulpina (L.) Hue Nouv. Archiv. du Museum ser. 4. 1:57. 1899.

Lichen vulpinus Linn. Sp. P1. 1155. 1753.

Evernia vulpina (L.) Ach.

Thallus tufted, lemon yellow ( $R$ ) or various shades of yellow-green (R), erect, rarely pendulous, radial, rather stiff, subterete, angular, rugose, lacunose, dichotomously much branched, branches divergent, wider at the base, acuminate, tips often forked, frequently sorediate. Apothecia large, terminal or subterminal, $15-20 \mathrm{~mm}$. in diameter, pedicellate; disk chestnutbrown $(\mathrm{R})$, concave; thalline margin wrinkled, sometimes fringed with a number of fiber-like branches; spores hyaline, ellipsoid, nonseptate, 6.0$11.0 \times 4.0-5.3 \mu$.

On trees and fallen branches of trees, more rarely on fence rails: San Juan Co.: San Juan Island, Trout Lake, 1940, 3218. Thurston Co.: Gate, 1940, 2958. Kitsap Co.: Hidden Ranch near Bremerton, 213 ft., 1930, 1879. Pierce Co.: Mt. Rainier, Summerland, 6500 ft., 1931, 685. Yakima Co.: Rattlesnake Canyon, 2000 ft., 1931, 1539. Chelan Co.: Merritt, 2186 ft., 1931, 1071. Okanogan Co.: Wauconda Summit, 4310 ft., 1931, 13996. Spokane Co.: Mt. Spokane, 5808 ft., 1931, 1488. Whitman Co.: Tekoa Mountains, $2900 \mathrm{ft}$., 1931, 1605. Columbia Co.: Blue Mountains, $3500 \mathrm{ft}$., 1931, 1624. San Juan Co.: San Juan Island, Mt. Alice, 1906, Fink 433 (W). Clallam Co.: Port Angeles, Birds, 1914, Foster 266 (W). Klickitat Co.: Goldendale, 1909, Foster 1157 (W).

A very common and conspicuous species east of the Cascades, wherever Pinus ponderosa grows. A rare lichen west of the Cascades. Plate VII, D.

59. Alectoria Ach. Lichenogr. Univers. 120. 1810.

Thallus straw color, greenish gray, or almost black, fruticose, filamentous, tufted, subterete to terete, erect or pendent, forming tangled hair- 
like masses, cortex of densely packed longitudinal hyphae surrounding the algal layer, and medulla of loosely woven hyphae, center of cylinder often hollow, attached by a holdfast. Apothecia rare, circular, lateral to subterminal, immersed, sessile, or pedicellate; thalline margin nearly always entire; hypothecium hyaline; paraphyses, branched and anastomosing; asci 2 -8-spored, spores usually hyaline, sometimes brown, nonseptate.

A. Thallus light colored, grayish green to straw color, rarely light brown; spores brown.

6. A. sarmentosa

A. Thallus dark colored, usually some shade of brown; spores hyaline.

B. Thallus rather stiff and wiry, erect or prostrate, rarely pendent.

C. Apothecia abundant; thallus rarely sorediate.

D. Thallus dark brown throughout; margins of apothecia fibrillose. 4. A. oregana

D. Thallus light colored at base, dark at the tips; margins of apothecia not fibrillose. 5. A. nigricans

C. Apothecia not abundant; thallus abundantly sorediate.

1. A. chalybeiformis

B. Thallus usually pendent, rarely erect or prostrate.

E. Thallus tufted, blackish brown, rarely white sorediate...3. A. jubata

E. Thallus pendulous, brown, more rarely blackish brown, rarely yellow sorediate.

2. A. Fremontii

1. Alectoria chalybeiformis (L.) Röhl. Deutschl. Fl. 3, Abt. 2:137. 1813. Lichen chalybeiformis Linn. Sp. P1. 1155. 1753.

Alectoria jubata var. chalybeiformis (L.) Ach.

Thallus filamentous, various shades of brown, tufted, short, smooth, stiff and wiry, erect, spreading, procumbent or rarely pendulous, terete to subterete, sparingly dichotomously branched, branches divaricate, often spinulose, sparingly fibrillous, usually abundantly sorediate; sterile in the state, doubtful if ever collected fruiting. Howe (1911) gives a maximum length of $12 \mathrm{~cm}$.

On trees and fallen branches, on wooden fences, more rarely on a layer of moss or soil over rock: Pierce Co.: White River Camp, $3900 \mathrm{ft} ., 1940$, 3096; trail to Mowich Lake, 3500 ft., 1940, 3073b. Kittitas Co.: Easton, 2168 ft., 1931, 837. Klickitat Co.: Goldendale, 2000 ft., 1931, 1703. Whitman Co.: Pullman, 2550 ft., 1926, G. N. Jones 3057 (W).

2. Alectoria Fremontii Tuck. Am. Jour. Arts \& Sci. ser. 2. 25:422. 1858.

Thallus dull olive brown to reddish brown, rarely almost black, long, pendulous, rather fine and pliant, terete to subterete, irregularly much branched, forming long tangled hair-like masses, main branches flexuous and lacunose, secondary branches elongated, flexuous with occasional greenish or sulphur colored soredia. Apothecia rare, marginal, adnate to sessile, 2-4 mm. in diameter; disk yellow to yellowish or green, flat to convex, pruinose; thalline margin thin, entire, finally disappearing; asci 8- 
spored; spores hyaline, ovoid ellipsoid, nonseptate, 3.9-7.0 × 3.3-5.0 $\mu$. Howe (1911) gives a maximum length of $45 \mathrm{~cm}$.

On trees and fallen branches, usually conifers : Pierce Co.: Mt. Rainier, Berkeley Park, 6000 ft., 1940, 3044; Mt. Rainier, Summerland, 6500 ft., 1931, 686. Yakima Co.: Bumping Lake, 3400 ft., 1931, 934. Kittitas Co.: South Cle Elum, 3000 ft., 1931, 877. Chelan Co.: Stehekin Valley, 1140 ft., 1931, 1288. Okanogan Co.: Twisp Summit, 6066 ft., 1931, 1317. Spokane Co.: Mt. Spokane, 5808 ft., 1931, 1484. Washington Territory, Suksdorf (T). Ferry Co.: Republic, 1912, Foster 2297 (W). Klickitat Co.: Goldendale, 1909, Foster 1154 (W).

If sterile and without soredia, this is a hard species to determine; however, the flexuous and lacunose main branches are helpful characters.

3. Alectoria jubata (L.) Ach. Lichenogr. Univers. 592. 1810.

Lichen jubatus Linn. Sp. Pl. 1155. 1753.

Thallus greenish-gray, brown, or almost black, stiff or somewhat flexible, prostrate or pendulous, terete, abundantly dichotomously branched, main branches flattened, secondary branches very slender, filiform, twisted in all directions, becoming much elongated, occasionally white sorediate. Howe (1911) reports a maximum length of $50 \mathrm{~cm}$. I have seen no fruiting specimens that were collected in the state.

On trees, old wood, or soil: Grays Harbor Co. : Lake Quinault, 300 ft., 1931, 1211. Pierce Co.: Silver Springs, 2670 ft., 1931, 720. King-Kittitas Co.: Snoqualmie Pass, 3010 ft., 1931, 814a. Yakima Co.: Lodge Pole Pine Camp, 3500 ft., 1940, 3304. Pierce Co.: Mt. Rainier, Mowich Lake Trail, 3500 ft., 1940, 3073. Okanogan Co.: Twisp Trail, 5000 ft., 1931, 1312. Columbia Co.: Blue Mountains, $3500 \mathrm{ft} .$, 1931, 1617. Washington Territory, 1882, Suksdorf 130 (T). Skagit Co.: Hamilton, 1905, Foster (W). Pierce Co. : Rainier National Park, $5000 \mathrm{ft} ., 1924$, J. M. Grant (F).

If sterile and without soredia, this too, is a hard species to determine.

4. Alectoria oregana Tuck. in Nyl. Lich. Jap. Obs. 2. 104. 1890.

Thallus reddish-brown to black, often paler towards the base, stiff and wiry, smooth and shiny, erect or pendulous, terete to somewhat flattened and channeled, much dichotomously branched, branches rather short and stout, tapering to the sharp pointed tips. Apothecia common, marginal or subterminal, 2-6 $\mathrm{mm}$. in diameter, adnate to sessile; disk dark brown, concave to convex; thalline margin shiny, rough to tuberculate with prominent fibrils; asci 8-spored; spores hyaline, ovoid to subglobose, nonseptate, 5.9$8.0 \times 3.9-5.0 \mu$.

On trees, usually conifers, and fence rails: Pierce Co.: Mt. Rainier, Paradise Park, 5550 ft., 1928, 1881. Ferry Co.: Mt. Gibraltar, near Republic, $3100 \mathrm{ft}$., 1940, 3172. Washington Territory, 1882, Suksdorf 72 (T). Klickitat Co.: Goldendale, 1909, Foster 1159 (W). Ferry Co.: Republic, 3000 ft., 1912, Foster 2295 (W). 
The abundant apothecia and the fibrillose margins make this one of the few easily determined species of Alectoria.

5. Alectoria nigricans (Ach.) Nyl. Lichenogr. Scand. 71. 1861.

Cornicularia ochroleuca var. nigricans Ach. Lichenogr. Univers. 615. 1810.

Alectoria ochroleuca var. nigricans (Ach.) Körb.

Thallus yellow or some shade of brown near the base, at least the upper one-third black, erect or prostrate, rigid, subterete to somewhat channeled, irregularly dichotomously branched, branches rather short, tips furcate. Apothecia rare, marginal or terminal, 1-2 mm. in diameter, sessile; disk concolorous with the thallus, or chestnut brown ( $R$ ), concave to convex; thalline margin at first nearly hyaline, at length becoming brown, crenulate, finally disappearing; asci 2-3-spored; spores hyaline, ellipsoid, nonseptate, 23.1-30.0 x 13.2-19.8 $\mu$. Howe (1911) reports a maximum length of $10 \mathrm{~cm}$.

On soil or rocks: Clallam Co. : between Mt. Angeles and Port Angeles, fog belt, 1911, Foster (F).

This species can easily be distinguished by the two-colored thallus, the lower part some shade of yellow or brown, at least the upper third almost black. Herbarium specimens after being collected for some time color the paper red.

6. Alectoria sarmentosa Ach. Lichengr. Univers. 595. 1810.

Lichen sarmentosus Ach. Liljebl. Utkast. Svensk. F1. 427. 1792.

Alectoria ochroleuca var. sarmentosa Nyl.

Thallus grayish-olive (R), straw yellow, dark brown, or almost black at times, smooth and dull, long and pendulous, sometimes prostrate, stiff and wiry like coarse hair, occasionally dilated, often papillate and pitted, terete to subterete, sparingly dichotomously branched below, abundantly branched towards the terete tips. Apothecia not uncommon, marginal, 1-6 $\mathrm{mm}$. in diameter, sessile; disk yellow or dark brown, concave to flat; thalline margin disappearing; asci 8-spored; spores brown, ellipsoid, nonseptate, $29.7-42.9 \times 14.0-23.1 \mu$. Howe (1911) reports the maximum length of the thallus $45 \mathrm{~cm}$.

On trees, widely distributed throughout the state from sea-level to 5000 ft.: Pacific Co.: Long Beach, 1931, 1024a. San Juan Co.: Cypress Island, 1928, 2116. Kitsap Co.: Hidden Ranch near Bremerton, 213 ft., 1940, 3278. Pierce Co.: Mt. Rainier, trail to Seattle Park, 4000 ft., 1940, 3054; Mt. Rainier, Berkeley Park, 6000 ft., 1940, 3041. King-Kittitas Co. : Snoqualmie Pass, 3010 ft., 1931, 815. Yakima Co.: Bumping Lake, 3400 ft., 1931, 935. Kittitas Co.: Easton, 2168 ft., 1931, 841. Klickitat Co.: Goldendale, 2000 ft., 1931, 1704a. Columbia Co.: Blue Mountains, 3500 ft., 1931, 1616. Pend Oreille Co.: Metaline Falls, 2600 ft., 1931, 1477. Whatcom Co.: Ruth Mountain, 5500 ft., 1928, L. Sunquist 2117. Clallam Co.: Port Angeles, 1912, Foster 2615 (W). San Juan Co.: Orcas Island, 
Mt. Constitution, 1906, Fink 317 (W). Jefferson Co.: Olympic Mountains, Queets River Valley, $5000 \mathrm{ft.,}$ 1907, Frye 37 (W).

The only long and extensive, light colored Alectoria. It is sometimes mistaken for an Usnea, because of the grayish or yellowish green color, but since there is no characteristic central cord in the medulla, it can easily be distinguished from the Usneas. Plate VIII, B, C.

\section{Cornicularia Ach. Vetensk.-Akad. Nya Handl. 15 :259. 1794.}

Thallus chestnut-brown ( $R$ ) or black, fruticose, cartilaginous, tufted, erect or procumbent, terete or flattened, much branched dichotomously or irregularly, tips sharp pointed, often furcate, somewhat channeled, or lacunose, differentiated into a plectenchymatous cortex, surrounding a thin algal layer, and a loose cottony (tow) medulla, often hollow in the center, loosely attached to the substratum by holdfast. Apothecia circular, marginal, subterminal, or terminal, small to large, sessile; disk the same color as the thallus, concave to convex; thalline margin often shiny, smooth, crenulate, or toothed; hypothecium hyaline; paraphyses usually unbranched, conglutinate and septate; asci 8-spored; spores hyaline, nonseptate.

A. Apothecia rare; tips spinulose.

3. C. tenuissima A. Apothecia abundant; tips not spinulose.

B. Branchlets fastigiated, margins of apothecia blunt toothed, tips of thallus not divided.

2. C. normoerica

B. Branchlets not fastigiated, margins of apothecia jagged, tips of thallus much divided.

1. C. californica

1. Cornicularia californica (Tuck.) DReitz, Arkiv för Bot. 20: No. 11 33 and 41. 1926.

Cetraria californica Tuck. Am. Jour. Arts and Sci. Ser. 2. 28:203. 1859.

Thallus light drab $(R)$, grayish olive $(R)$, or black, fruticose, short, erect, tufted, cartilagineous, lacunose, somewhat channeled, dull, irregularly dichotomously branched, flat or subterete, tips much divided, fertile branches thickened near the tips, below paler. Apothecia 1.5-5.0 mm. in diameter, terminal to subterminal, sessile, appendiculate; disk dark green, dark purple or black, flat to convex, surface uneven; thalline margin smooth, fringed, toothed, soon excluded by the disk; spores hyaline, ellipsoid, nonseptate, 7.0-8.0 $\times$ 4.5-5.0 $\mu$.

On trees, shrubs, and fences: Grays Harbor Co.: Westport, 1908, Foster (F). Merrill Exs. No. 82. Determined as Alectoria californica (Fink) Merrill. Plate VIII, D. Plate IX, A.

The dark-colored or almost black, erect thallus, with often abundant apothecia, makes this rather an easy species to recognize.

2. Cornicularia normoerica (Gunn.) DRietz, Arkiv för Bot. 20, No. 11. 32 and 39. 1926. 
Lichen normoericus Gunn. Fl. Norveg. 2:123. 1776.

Cetraria tristis (Web.) Fries.

Thallus black, sometimes brownish towards the base, small, fruticose, erect, tufted, stiff, rigid, and tenacious, sparingly branched, branches repeatedly divided towards the tips, margins toothed. Apothecia numerous, subterminal, 1-6 mm. in diameter, sessile; disk black to very dark brown, flat to convex; thalline margin smooth or toothed; spores hyaline, ellipsoid, nonseptate, 8.4-10.0 × 4.5-5.0 $\mu$.

On rocks in the alpine life zone: Pierce Co.: Mt. Rainier National Park, Burroughs Mountain, 7300 ft., 1934, 2485. Olympic Mountains, Hurricane Ridge, $6000 \mathrm{ft}$., A. H. Smith 2394 (M). Tatoosh Range, Pinnacle Peak, 7200 ft., 1909, Foster 1055 (F). Plate IX, C.

3. Cornicularia tenuissima (L.) Zahlbr. Annal. Naturh. Museum Wien. $42: 64.1928$.

Lichen tenmissimus Linn. Sp. P1. 1145. 1753.

Cetraria aculeata (Schreb.) Fries.

Thallus pale brown, chestnut-brown $(R)$, or black, fruticose, erect, or somewhat prostrate, stiff and rigid, often shiny, slightly lacunose, subterete to flattened, almost hollow with some fine cottony medulla remaining, much divaricately branched, becoming fastigiate, branches furcate, tips spinulose. Apothecia rare, not seen on any Washington specimens, subterminal, $2-8 \mathrm{~mm}$. in diameter, sessile; disk chestnut-brown $(R)$ or dark brown, concave to convex, surface irregular; thalline margin toothed or spiny; spores hyaline, ellipsoid, nonseptate, 6.5-10.0 x 3.3-4.0 $\mu$.

On rock or on thin layer of soil over rock: San Juan Co. : Orcas Island, Mt. Constitution, 2500 ft., 1928, 2082; San Juan Island, near Trout Lake, 1940, 3225; Brown Island, 1940, 3202; Waldron Island, 1906, Fink 214 (F). Ferry Co.: Republic, Maye's Butte, 1913, Foster 2284 (W).

The only species of Cornicularia that is at all plentiful in the state. It is rather abundant in the few regions in which it has been collected. Plate IX, D.

\section{Ramalina Ach. Lichenogr. Univers. 122. 1810.}

Thallus various shades of greenish gray, yellowish green, pale yellow or white, fruticose, cartilaginous, stiff and rigid, erect or rarely pendent, flattened, subterete or terete, much branched, more or less dichotomously, differentiated into a plectenchymatous cortex, surrounding an algal layer, and an abundant cottony medulla, some of the hyphae uniting to form strong cords, or hyphae very scarce and attached to the inner surface of the cortex, center hollow, firmly attached to substratum. Apothecia circular, terminal or marginal, sessile or subpedicellate; disk usually the same color as the thallus, concave to convex; thalline margin sometimes disappearing; hypothecium hyaline; paraphyses rarely branched, agglutinated; asci 8-spored; spores hyaline, straight or curved, 1-septate or rarely 3-septate. 
A. Thallus sorediate or soraliate.

B. White powdery soredia, on tips only.

C. Thallus delicately branched, tips not bursting open....8. R. Roesleri

C. Thallus rather coarsely branched, tips bursting open.

6. R. pollinaria

B. Thallus soraliate, usually on the margins, never broadly expanded, sometimes channeled but not lacunose.

4. R. farinacea

A. Thallus neither abundantly sorediate or soraliate.

D. Thallus a lace-like network, long and pendulous.

7. R. reticulata

D. Thallus not a lace-like network, not long and pendulous.

E. Medulla floccose, bursting through cracks in the cortex.

1. R. ceruchis

E. Medulla not as above.

F. Thallus fistulate.

G. Thallus forming small cushion-like masses, slightly inflated.

3. R. dilacerata

G. Thallus larger, more spreading, apothecia abundant, attached just below the deflexed tips of the branches.......2. $R$. digitata

$F$. Thallus not fistulate, membranaceous, becoming lacunose, apothecia marginal or subterminal, rather abundant.

5. R. Menziesii

1. Ramalina ceruchis (Ach.) De Not. Giorn. Bot. Ital. 2:218. 1846.

Parmelia ceruchis Ach. Meth. Lich. 260. 1803.

Thallus honey colored ( $R$ ), straw yellow ( $R$ ), or sometimes rufescent, black towards the base, lobes tufted, erect, subterete or flattened, smooth or deeply pitted and wrinkled, punctate with large black spermagonia, simple or sparingly branched, tips attenuated. Apothecia not uncommon, marginal, 1-6 $\mathrm{mm}$. in diameter, sessile; disk buff or concolorous with the thallus, often pruinose, concave to convex; thalline margin crenulate; spores hyaline, oblong-ellipsoid, straight or slightly curved, nonseptate, 12.0-16.0 x 3.0-5.0 $\mu$.

On trees, old wood, and rocks: San Juan Co. : San Juan Island, Kanaka Bay, Foster, reported by Merrill (1911). Clallam Co.: Dungeness, 1913, Foster 2533 ( F).

It always grows near the ocean. Older plants and those in herbaria are very often partly covered with a white or grayish white cottony floccose down, resembling mold; this is caused by the medullary hyphae bursting through the cracks in the cortex.

2. Ramalina digitata Mey. et Flw. Nova Acta Acad. Leopoldin.-Carolin. 19, Suppl. 212. 1843.

Ramalina geniculata Hook. f. et Tayl., London Jour. Bot. 3. 655. 1844. Ramalina pusilla var. geniculata Tuck. Syn. N. Am. Lich. 26. 1882.

Ramalina inflata Hook. f. and Tayl. 
Thallus grayish olive $(R)$ or straw-colored $(R)$, fruticose, lobes short, tufted, rigid, smooth and shiny, subterete or somewhat flattened, with longitudinal ridges, fistulose and frequently perforate, much dichotomously branched, the tips somewhat digitately divided. Apothecia terminal or subterminal, 1-3 mm. in diameter, sessile; disk pale flesh colored or pale yellow, pruinose, concave to flat, attached just below the deflected tips; thalline margin smooth, spores hyaline, oblong to oblong-fusiform, straight or slightly curved, 1-septate, 10.0-15.0 x 4.0-6.0 $\mu$.

On trees or wooden fences: San Juan Co.: Brown Island, 1940, 3261. Pacific Co.: Chinook, 1940, 2839; Long Beach, 1940, 2753. Wakhiakum Co.: Cathlamet, 1940, 2905. Kitsap Co.: Hidden Ranch, near Bremerton, 213 ft., 1930, 1888. White Salmon River, 1882, Herbarium of C. F. Faxon (F).

This species can be recognized easily by its erect, fistulose thallus, and the subterminal apothecia, attached just below the sharp pointed bent tips of the thallus branches.

3. Ramalina dilacerata (Hoffm.) Wainio, in Meddel. Soc. Fauna et Fl. Fenn. 14:21. 1886.

Lobaria dilacerata Hoffm. Deutschl. F1. 140. 1796.

Ramalina minuscula Nyl.

Thallus grayish olive $(R)$ or straw colored $(R)$, very small, tufted in cushion-like masses, not exceeding $10 \mathrm{~mm}$. in length, Howe (1914), somewhat rigid, flattened or subterete, shiny, inflated, frequently perforated, dichotomously branched, tips forked, attenuated, maximum width $2 \mathrm{~mm}$. Apothecia terminal, 1-2 mm. in diameter, sessile; disk waxy looking, flesh colored, pruinose, concave to convex, thalline margin finally disappearing; spores hyaline, usually straight, oblong-ellipsoid, 1-septate, 9.0-16.0 x 4.0-6.0 $\mu$.

On trees: Thurston Co.: Gate, 1940, 2839; Gate, 1912, Foster (F).

4. Ramalina farinacea (L). Ach. Lichenogr. Univers. 605. 1910.

Lichen farinaceus Linn. Sp. P1. 1146. 1753.

Ramalina calicaris var. farinacea (L.) Rabh.

Thallus grayish olive (R), cream color $(R)$, or honey yellow (R), erect or pendulous, tufted, stiff and rigid, smooth, lobes linear, flattened or subterete sometimes filiform, attenuate, rarely channeled or pitted, sparingly, or abundantly branched, rather abundantly white sorediate. Apothecia rare and inconspicuous, marginal, sessile; disk buff or concolorous with the thalline margin, which finally disappears; spores hyaline, oblongellipsoid, straight or rarely curved, 1-septate, 13.2-15.0 x 4.0-7.0 $\mu$.

On trees, old wood, and rarely on rock: Pacific Co.: Long Beach, 1940, 2934; Loomis Lake, 1940, 2842: Chinook, 1931, 801. Wahkiakum Co.: Cathlamet, 1940, 2857. Clallam Co.: Cedarville, 300 ft., 1940, 2890. San Juan Co.: Friday Harbor, 1928, 1885; Brown Island, 1931, 2083. Grays 
Harbor Co.: Westport, 1908, Foster 614 (F) ; Copalis, 1911, Foster 1458 (W). Clallam Co.: Dungeness, 1913, Foster 2534 (W). Thurston Co.: Tenino, Frye (W). Kittitas Co.: Ellensburg region, 1935, Sister Marion Loretta (WTU). Spokane Co.: Liberty Lake 1909, T. A. Bon$\operatorname{ser}$ (WTU).

The most common species of Ramalina in the state. Plate $\mathrm{X}, \mathrm{D}$.

5. Ramalina Menziesii Tuck. Syn. Lich. N. Eng. 12. 1848.

Ramalina leptocarpha Tuck. Am. Jour. Arts and Sci. Ser. 2, $25: 423$, 1858.

Thallus olive-buff (R), straw yellow (R), or honey colored (R), tufted, erect or pendulous, flattened, membranaceous, thin, rigid, rather ribbon like, flexuous, channeled, becoming lacunose, sparingly branched, puberulent, rarely sorediate, tips of branches acuminate. Apothecia circular, marginal or subterminal, 1-6 mm. in diameter, subpedicellate; disk concolorous with the thallus, concave to convex; thalline margin entire, sometimes disappearing; spores hyaline, oblong-ellipsoid, straight or often curved, nonseptate, 14.0-18.0 × 5.5-8.0 $\mu$.

On trees or old wood: San Juan Co.: Friday Harbor, 1906, Fink and L. Clark (M) ; Wasp Island, 1906, Fink 197 (M).

The broadly expanded lacunose thallus helps to distinguish this lichen. It is very rare in Washington, but common farther south, especially in California.

6. Ramalina pollinaria (Liljebl.) Ach. Lichenogr. Univers. 608. 1810.

Lichen calicaris C. pollinaris Liljebl. Utkast Svensk. F1. 426. 1792.

Thallus cream color $(\mathrm{R})$, honey yellow $(\mathrm{R})$, or greenish gray, rather membranaceous, tufted, flaccid, lobes flattened, narrow, wrinkled, channeled, branching irregularly, branches rather short, tips broken and torn, powdery sorediate, especially at the bursting tips. Apothecia rare, subterminal, 0.8-3.0 $\mathrm{mm}$. in diameter, sessile; disk concolorous with the thallus, concave; thalline margin rarely disappearing; spores hyaline, oblong-ellipsoid, straight or slightly curved, 1-septate, 9.0-13.0 x 4.0-6.0 $\mu$.

On trees, more rarely on rocks: San Juan Co.: Brown Island, 1940, 3204; San Juan Island, Kanaka Bay, 1907, Foster 5591/2 (W), determined as Ramalina pollinaria (Ach.) Nyl. form humilis Ach. Clallam Co.: Dungeness, 1913, Foster 2535 (F).

7. Ramalina reticulata (Noedh.) Krmphbr. Geschichte der Lich. 2:617. 1869.

Lichen reticulatus Noedh. Jour. für Bot. $1: 238.1800$.

Thallus buffy olive $(\mathrm{R})$, chamois $(\mathrm{R})$, or grayish green, alike on both sides, compressed, greatly elongated, pendulous, never tufted, rather stiff and rigid, much branched, lobes flattened, rarely subterete, narrow or dilated, sometimes longitudinally channeled, finally perforated and forming tangled mats of a coarse or delicate net-work. Apothecia marginal, small, 
inconspicuous, 1-3 $\mathrm{mm}$. in diameter, sessile; disk concolorous with the thallus, or salmon-color ( $R$ ), concave to convex; thalline margin disappearing; spores hyaline, oblong-ellipsoid to ellipsoid, straight or slightly curved, 1-septate, $11.5-16.5 \times 3.9-5.9 \mu$.

On trees and old wood: San Juan Co.: Brown Island, 1928, 1884; San Juan Island, Pt. Caution, 1940, 3271. Clallam Co.: Port Angeles, 1911, Foster 1813 (W) ; Dungeness, 1913, Foster 2536 (F). Wahkiakum Co.: Cathlamet, 1907, Foster 503 (F). Grays Harbor Co.: Montesano, 1919, J. M. Grant (F); Westport, 1908, Foster (F).

Readily determined because of its net-like structure. It has never been collected many miles away from the ocean. Plate X, A, B, C.

8. Ramalina Roesleri (Hochst.) Nyl. Bull. Soc. Linn. Normand. ser 2, $4: 165.1870$.

Ramalina farinacea var. Roesleri Hochst. in Schaer., Enum. Critic. Lich. Eur. 9. 1850.

Ramalina pollinariella Nyl.

Thallus grayish green, Isabella color $(R)$, apricot buff $(R)$, or tawnyolive (R), small, never more than $5 \mathrm{~cm}$. long, Howe (1914), erect, rigid, tufted, somewhat shiny, much branched irregularly, lobes subterete to flattened, tips fine and delicate, branched like a tree, often sorediate, only sterile specimens from the state have been seen by the writer.

On trees: Pacific Co.: Long Beach, 1940, 2754.

Apparently a rare species, perhaps overlooked because of its small size.

62. Usnea (Dill) Adans., Fam. P1. 2:7. 1763.

Thallus various shades of greenish gray or grayish green, yellowish green or pale yellow, fruticose, erect or pendulous, terete or sometimes angular, stiff, and often thread-like, smooth to often rough scurfy, papillate or sorediate, usually much branched irregularly, often forming entangled mats, horny cortical layer of closely interwoven hyphae completely surrounding a thin algal layer and cottony medulla with a central medullary cord of strong thick walled hyphae, no rhizoids, but attached to the substratum by a holdfast. Apothecia circular, terminal or lateral, pedicellate; usually with thalline margin colored like the thallus, more or less entire; hypothecium hyaline; paraphyses agglutinated, branched and septate, asci 8 -spored, spores hyaline, nonseptate.

A. Medullary cord treated with IKI, turns blue immediately.

5. U. longissima

A. Medullary cord treated with IKI, no color change.

B. Thallus rather short, erect, shrub-like, sorediate. 4. U. hirta

B. Thallus longer, more or less pendulous, rarely sorediate.

C. Cortex lacunose, seamed and ridged, not papillate....1. U. cavernosa C. Cortex smooth or papillate. 
D. Fibrils lacking or very sparse and tortuous 6. U. plicata

D. Fibrils numerous.

E. Cortex densely papillate, fibrils branched, white medulla showing through cracks in the cortex, medulla shows no color change with $\mathrm{KOH}$.

2. U. ceratina

E. Cortex sparsely papillate, fibrils rarely branched, white medulla not showing through cracked cortex, medulla yellow then turning red with $\mathrm{KOH}$; all specimens do not show this color change.

3. U. dasypoga

1. Usnea cavernosa Tuck. in Agassiz, Lake Superior 171. 1850.

Thallus greenish gray, pendulous, elongated, terete or angular, often lacunose, sometimes seamed and ridged, cortex broken in partial or complete rings, but rarely exposing the medulla, main branches like very coarse hair, secondary branches finer, irregularly dichotomously branched, bearing long, slender tangled fibrils. Apothecia marginal, 3-5 mm. in diameter, pedicellate; disk concolorous with the thallus or flesh colored, flat; thalline margin often bearing fibrilloid branches; spores hyaline, ovoid ellipsoid, nonseptate, $9.0-11.0 \times 5.5-7.0 \mu$.

On trees: Reported from Washington by Fink (1935), but I have never studied a specimen collected in the state.

The terete, ridged and sometimes lacunose main branches distinguish this from any other species of Usnea.

2. Usnea ceratina Ach. Lichenogr. Univers. 619. 1810.

Usnea barbata var. ceratina (Ach.) Schaer.

Thallus grayish green, honey yellow (R), or rufescent, pendulous, flexible, thickly papillate, at times sorediate, cortex often broken into rings exposing the white medulla, branches coarse, terete, elongated, usually much divided dichotomously, thickly beset with curly fibrils that are often branched. Apothecia lateral or subterminal, 2-9 mm. in diameter, pedicellate; disk concolorous with the thallus or pale olive-buff $(R)$, flat; thalline margin entire or bearing fibrilloid branches; spores hyaline, ovoid ellipsoid, nonseptate, 4.5-8.0 × 4.5-6.0 $\mu$. Medulla shows no color change with $\mathrm{KOH}$. Plate XI, C.

On trees: Pacific Co.: Long Beach, 1928, 2125a; Loomis Lake, 1931, 747. Wahkiakum Co.: Cathlamet, 1940, 2858. San Juan Co.: San Juan Island, Friday Harbor, 1940, 3240. Island Co.: Whidbey Island, 1931, 1779. Clallam Co.: Port Angeles, 1911, Foster (F). Spokane Co., 1906, J. A. Bonser (F). Clallam Co.: Sequim, 1915, J. M. Grant (F).

3. Usnea dasypoga (Ach.) Röhl. Deutschl. Fl. 3, Abt. 2:144. 1813.

Usnea plicata var. dasypoga Ach., Meth. Lich. 312. 1803.

Usnea barbata var. dasvpoga Ach. Lichenogr. Univers. 624. 1810. 
Thallus grayish green, honey yellow ( $R$ ), or straw yellow (R), rarely rufescent, main branches blackening toward the base, elongated and pendulous, terete, slender, rough, more or less papillate, rather sparingly branched, thickly beset with short, spreading, rarely branched fibrils, cortex often broken in rings, but rarely exposing the white medulla to any marked degree. Apothecia rare, marginal or subterminal, 1-5 mm. in diameter; disk concolorous or very pale olive-buff ( $R)$, flat; thalline margin entire or bearing fibrilloid branches; spores hyaline, ellipsoid, nonseptate, 7.0-10.0 x 4.0-7.0 $\mu$. Medulla yellow with $\mathrm{KOH}$, later turning red, with some specimens these color changes do not appear.

On trees and rarely on old wood: Pacific Co.: Chinook, 1940, 2795; Loomis Lake, 1940, 2841. San Juan Co.: Brown Island, 1940, 3258; Orcas Island, Mt. Constitution, 2500 ft., 1928, 2122. Clallam Co.: Elwha River Trail, 500 ft., 1940, 2866a. Mason Co. : Shelton, 1931, 1224. San Juan Co.: San Juan Island, 1906, Fink 443 (W).

\section{Usnea hirta (L.) Wigg., Prim. F1. Holstat. 91. 1780. \\ Lichen hirtus Linn. Sp. P1. 1155. 1753. \\ Usnea barbata hirta (L.) Fries, Lichenogr. Eur. Reform. 18. 1831.}

Thallus grayish green or straw yellow ( $R$ ), rufescent at times, usually erect, caespitose, terete, rather stiff and rigid, short, rarely exceeding $8 \mathrm{~cm}$. in length, main branches crowded, secondary branches dichotomously arranged, slender, frequently long and curving, fibrils abundant, farinosesorediate. Apothecia often degenerate, rare, marginal or subterminal, 3-6 $\mathrm{mm}$. in diameter; disk dirty flesh color, flat; thalline margin usually bearing fibrilloid branches; spores hyaline, ellipsoid, nonseptate, 5.0-7.0 x 8.0$10.0 \mu$.

On trees, shrubs, and wooden fences: Pacific Co.: Long Beach, 1940, 2933; Chinook, 1940, 2802. Wahkiakum Co.: Cathlamet, 1940, 2904. San Juan Co.: Brown Island, 1940, 3260. Mason Co.: Shelton, 1931, 1220. Kitsap Co.: Hidden Ranch near Bremerton, 213 ft., 1940, 3281a. Thurston Co.: Gate, 1940, 2963. King Co.: Woodinville, 1940, 3346; Edgewood, 300 ft., 1940, 3085. Grays Harbor Co.: Westport, 1908 Foster (W), determined as U. barbata by H. E. Hasse. Spokane Co.: Liberty Creek, 1910, T. A. Bonser 57 (W). Wahkiakum Co.: Cathlamet, 1907, Foster $502(\mathrm{~W})$.

This species is easily distinguished by its small size, shrubby nature of growth, lack of apothecia, and abundance of soredia. Plate XI, A.

\section{Usnea longissima Ach. Lichenogr. Univers. 626. 1810.}

Thallus grayish green or straw yellow $(R)$, pendulous, main branches greatly elongated, terete to slightly flattened, sparingly dichotomously branched, with numerous simple horizontal fibrils, surface scurfy, medulla turns blue with IKI. Apothecia very rare in the United States. No Washington specimens seen fruiting. 
On trees: Grays Harbor Co.: Lake Quinault, 300 ft., 1931, 1204a. Pacific Co.: Chinook, 1940, 2796, 2835b. Clallam Co.: Sequim, 1915, J. M. Grant (F), this specimen was determined as Usnea ceratina by G. K. Merrill. Pierce Co.: Steilacoom, Washington Territory, 1865, Torrey (F). Thurston Co.: Gate, 1911, Foster 1934 (W). Lewis Co.: Mineral, 1905, Flett (F), vidi J. Motyka.

As the specific name indicates, this is the longest of the Usneas. The blue color reaction of the medulla with IKI is distinctive. Plate XI, B.

6. Usnea plicata (L.) Wigg. Prim. F1. Holsat. 91. 1780.

Lichen plicatus Linn. Sp. P1. 1154. 1753.

Usnea dasypoga var. plicata (L.) Cromb., Monogr. Brit. 1 :204. 1894.

Usnea barbata var. plicata (L.) Fries Lichenogr. Eur. Reform. 18. 1831.

Lichen barbatus Linn. Sp. P1. 1155. 1753.

Thallus grayish green or straw yellow ( $R$ ), elongated and pendulous, terete, main branches coarse at length intertangled, secondary branches irregularly dichotomous, fibrils lacking or sparse and tortuous, cortex rough, somewhat papillate, sometimes broken into rings, rarely exposing the white medulla. Apothecia marginal or subterminal, $1-3 \mathrm{~mm}$. in diameter, pedicellate; disk pale flesh color, often turning chestnut-brown $(R)$, concave to plane; thalline margin entire, spores hyaline, ellipsoid, nonseptate, $5.0-10.0 \times 4.0-8.0 \mu$.

On trees and rarely on old wood: Pacific Co.: Loomis Lake, 1940, 2840. Kitsap Co.: Hidden Ranch near Bremerton, 213 ft., 1930, 527. Clallam Co.: Port Angeles, 1911, Foster 1811 (F); Cape Flattery, A. H. Smith, 1897 (M).

63. Thamnolia Ach., in Schaer. Enum. Lich. Eur. 243. 1850.

Thallus fruticose, consisting of erect or prostrate, hollow podetia, with a well-developed plectenchymatous cortex surrounding an algal layer and a thin medulla, hollow center; horizontal thallus lacking. Apothecia not found in America.

1. Thamnolia vermicularis (Sw.) Schaer. Enum. Critic. Lich. Eur. 243. 1850.

Lichen vermicularis Sw., Meth. Muscor. 37. 1781.

Podetia pure white or ashy, erect or prostrate, short or somewhat elongated, sparingly branched, often forked toward the tip, subulate, hollow, terete to slightly flattened, podetia often densely clustered.

On soil : Pierce Co.: Mt. Rainier, Burroughs Mountain, 7300 ft., 1931, 603. San Juan Co.: Orcas Island, Mt. Constitution, 2500 ft., 1928, 2067; 1906, Fink 329 (W). Mason Co.: Mt. Elinor, 5500 ft., 1912, Foster 2098 (W). Olympic Mountains, 1917, J. M. Grant (F). Pierce Co.: Mt. Rainier, Table Mountain, 8000 ft., 1925, J. M. Grant 409 (F). 


\section{CALOPLACACEAE}

Thallus crustose, foliose, or squamulose, dorsi-ventral, little or no differentiation into definite layers, attached to the substratum by hyphal rhizoids, no true rhizoids. Apothecia circular, scattered, immersed, adnate or sessile, with either a thalline or a proper margin or both; asci 4-8-spored, usually 8-spored; spores hyaline, rarely nonseptate, usually polar-bilocular, sometimes 2- or 3-septate. Epithecium and thallus usually violet, purple, or some shade of red with $\mathrm{KOH}$.

Alga Protococcus.

A. Apothecium always has a thalline margin. 65. Caloplaca

A. Apothecia never has a thalline margin. 64. Blastenia

64. Blastenia Mass. Atti. Istit. Veneto ser. 2, 3:101. 1852.

Thallus crustose, smooth to powdery, scaly, squamulose, rarely areolate, usually no differentiation into layers. Apothecia small, circular, immersed, adnate, or sessile; with a proper margin; hypothecium hyaline; paraphyses unbranched, septate, enlarged, and rounded at the tips; asci 4-8-spored; spores hyaline, usually polar-bilocular, rarely nonseptate.

1. Blastenia atrosanguinea (Merrill) Fink Lich. Flora of the U.S. 353. 1935.

Lecanora atrosanguinea Merrill Ottawa Nat. 117. 1913.

Thallus pale greenish-gray, granulose, thin, scattered, scaly. Apothecia small, 0.4-1.0 mm. in diameter, sessile; disk reddish-brown to black, flat; proper margin concolorous, thin, entire or wavy; asci 8-spored; spores hyaline, ovoid-ellipsoid to oblong, polar-bilocular, 10.0-16.0 x 5.0-7.0 $\mu$.

On bark: Fink (1935) reports it from Washington. Herre (1917) reports it as not rare on Acer circinatum in Whatcom County.

\section{Caloplaca Th. Fr. Lich. Scand. 1:167. 1871-74.}

Thallus crustose, foliose, or squamulose, granulose or areolate, marginally lobed, rarely showing differentiation into definite layers. Apothecia minute, small to medium sized, circular, rarely immersed, adnate or sessile; with both proper and thalline margins, or with only thalline; hypothecium hyaline; paraphyses unbranched, septate, usually with enlarged rounded tips; asci 8-spored; spores hyaline, usually polar bilocular, some species nonseptate or 3-septate.

A. Thallus yellow, yellowish brown, or orange, rarely gray.

$B$. Thallus marginally lobed or subfoliose.

C. Lobes branched.

D. Lobes linear, much branched. 7. C. elegans

D. Lobes shorter and wider, marginally plicate or imbricate.

9. C. murorum 
C. Lobes not branched.

8. C. lobulata

B. Thallus not marginally lobed or subfoliose.

E. Thalline margin crenulate or wavy, rarely disappearing;

black hypothallus often visible.

1. C. aurantiaca

E. Thalline margin granulose, often disappearing; hypothallus white not often visible.

3. C. citrina

A. Thallus grayish white, olive brown or brown.

F. Hypothallus usually evident, bluish black to black.

G. Disk of apothecium rusty-red to black.

4. C. ferruginea

G. Disk of apothecium lemon-yellow to orange.

2. C. cerina

F. Hypothallus not usually evident.

$\mathrm{H}$. On soil, encrusting mosses or other lichens......5. C. jungermanniae

H. On rock, trees, or old wood......................................... 6. C. pyracea

1. Caloplaca aurantiaca (Lightf.) Th. Fr. Nova Acta Reg. Soc. Sci. Upsal. ser. 3. $3: 219.1861$.

Lichen aurantiacus Lightf. Fl. Scot. 2:810. 1777.

Placodium aurantiacum (Lightf.) Anzi.

Thallus well developed, lemon yellow $(R)$, orange, or rarely gray, crustose, continuous or areolate, warty and wrinkled, often bordering black hypothallus visible. Apothecia numerous, $0.5-1.5 \mathrm{~mm}$. in diameter, crowded, adnate to sessile; disk ochraceous-orange or brownish, flat to concave; thalline margin crenulate, sometimes becoming wavy, often raised, rarely disappearing; spores hyaline, ellipsoid, polar-bilocular, 10.0-13.5 $\mathrm{x}$ 4.9-6.6 $\mu$. Both epithecium and thallus purple with $\mathrm{KOH}$.

On bark, old wood, or rock: San Juan Co. : San Juan Island, English Camp, 1928, 2065 ; Pt. Caution, 1928, 2090. Clallam Co.: Port Angeles, 1914, Foster 2887 (W). Washington Territory, Columbia River, 1881, Suksdorf 24 (F).

2. Caloplaca cerina (Ehrh.) Th. Fr. Nova Acta Reg. Soc. Sci. Upsal. ser. $3,3: 218$. 1861.

Lichen cerinus Ehrh. in Hoffm. Descript. et Adumbrat. P1. Lich. 2:62. 1789.

Placodium cerinum (Ehrh.) Naeg.

Thallus grayish-white $(R)$ or brownish, smooth, crustose, thin or scurfy, becoming verrucose, effuse or chinky, and finally sub-areolate, a bluish-black hypothallus often visible. Apothecia numerous, $0.2-0.5 \mathrm{~mm}$. in diameter, adnate to sessile; disk lemon-yellow ( $R)$, ochraceous-orange $(\mathrm{R})$, or reddish, subpruinose, slightly concave to convex; thalline margin lighter or concolorous with the thallus, often the same color as disk, sometimes raised, entire or becoming wavy; spores hyaline, ellipsoid, polarbilocular, 10.0-16.5 x 6.2-9.9 $\mu$. Epithecium bright crimson with $\mathrm{KOH}$, color change of thallus is very slight with $\mathrm{KOH}$.

On bark, mosses, rarely on old wood and rock: San Juan Co.: Turn Island, 1928, 2108; San Juan Island, Pt. Caution, 1928, 2089. Kittitas 
Co.: Roslyn, 2800 ft., 1931, 1793. Chelan Co.: Rainbow Trail, 3500 ft., 1931, 1262. Stevens Co. : Boyd's, 1470 ft., 1931, 1451. Grays Harbor Co.: Pacific Co.: 1910, Foster (F). Wahkiakum Co.: Cathlamet, 1907, Foster 505 (F). Washington Territory, West Klickitat Co. : 1882, Comm. Sprague, collector not given, 558 (F). Spokane Co.: 1900, R. K. Beattie (F).

3. Caloplaca citrina (Hoffm.) Th. Fr. Nova Reg. Soc. Sci. Upsal. ser 3, $3: 218.1861$.

Verrucaria citrina Hoffm. Deutschl. Fl. 198. 1795.

Placodium citrinum (Hoffm.) Hepp.

Thallus lemon yellow $(R)$, crustose becoming granulose, scattered or compacted into a subareolate crust. Apothecia $0.2-0.5 \mathrm{~mm}$. in diameter, adnate; disk flat, wax yellow ( $R$ ) or ochraceous-orange ( $R)$; proper margin thin, often disappearing, thalline margin depressed, somewhat granulose; spores hyaline, ellipsoid, rather broad in the middle, polarbilocular, 9.9-14.0 × 4.9-6.6 $\mu$. Epithecium dull red with $\mathrm{KOH}$, color change of thallus is very slight with $\mathrm{KOH}$.

On rock, rarely on bark or old wood: Clallam Co.: Lake Sutherland, $590 \mathrm{ft} ., 1931,1019$.

4. Caloplaca ferruginea (Huds.) Th. Fr. Nova Acta Reg. Soc. Sci. Upsal. ser. 3, $3: 223.1861$.

Lichen ferrugineus Huds. Fl. Angl. 444. 1762.

Placodium ferrugineum (Huds.) Rabh.

Blastenia ferruginea (Huds.) Mass.

Thallus grayish-white, crustose, disappearing at times, thin and smooth, or thicker and areolate, or wrinkled and verrucose. Apothecia $0.2-1.0 \mathrm{~mm}$. in diameter, adnate to sessile; disk ferruginous (R), rusty-red, hazel (R), or black, flat to convex; proper margin prominent, thalline margin disappearing; spores hyaline, broadly ellipsoid, polar-bilocular, 11.0-19.0 x 7.0-9.0 $\mu$. Epithecium purple with $\mathrm{KOH}$, thallus faintly purple with $\mathrm{KOH}$.

On trees, old wood, and rocks: Klickitat Co.: Bickleton, Spring Canyon, $2500 \mathrm{ft} .$, 1931, 1656. Clallam Co.: Lake Crescent, A. H. Smith 13832 (M). San Juan Co. : Brown Island, 1906, Fink 470 (W). Snohomish Co.: Marysville, 1928, J. M. Grant (F), Det. Magmusson. King Co.: Alki Point, 1912, Fostcr 2182 (F). Clark Co.: Vancouver, 1881, Suksdorf (F).

5. Caloplaca jungermanniae (Vahl.) Th. Fr. Nova Acta Reg. Soc. Sci. Upsal. 3.2221 . 1861.

Lichen jungermanniae Vahl. Nat. Selak. Skr. 2. 29. 1792.

Placodium jungermanniae (Vahl.) Tuck.

Thallus grayish-white, granulose, or subsquamulose, very thin, encrusting mosses or other lichens. Apothecia numerous, crowded, 0.2-1.2 $\mathrm{mm}$. in diameter, sessile; disk ochraceous-orange $(R)$ or brownish, sub- 
pruinose; thalline margin at first thick, entire, or flexuous, later disappearing; spores hyaline, ellipsoid, polar-bilocular, $15.0-23.0 \times 8.0-12.0 \mu$. Epithecium dull red with $\mathrm{KOH}$, no color change of thallus with $\mathrm{KOH}$.

On mosses, lichens, or soil: Kittitas Co.: Roslyn, 2800 ft., 1931, 8996. Yakima Co.: Boulder Cave, 3000 ft., 1931, 1158. Whatcom Co.: Mt. Baker, 6000 ft., Herre (1917).

6. Caloplaca pyracea (Ach.) Th. Fr. Kgl. Sten. Vet.-Akad. Handl. 7 :25. 1867.

Parmelia cerina var. pyracea Ach. Meth. 1176. 1803.

Placodium pyraceum (Ach.) Anzi.

Placodium cerinum var. pyracemm (Ach.) Branth. \& Rostr.

Thallus olive-brown $(R)$ or grayish-white, scurfy-granulose, very thin, often disappearing. Apothecia numerous, often clustered, 0.2-0.7 mm. in diameter, adnate to sessile; disk yellow or orange, flat to convex; thalline margin paler than disk, often disappearing; spores hyaline, oblong ellipsoid, polar-bilocular, 9.9-2.2 x 4.0-7.0 $\mu$. Epithecium crimson with $\mathrm{KOH}$, no color change of the thallus with $\mathrm{KOH}$.

On bark, old wood, and rocks: Whitman Co. : Tekoa Mountains, 2900 ft., 1931, 1592. Clallam Co.: Dungeness, 1914, Foster 2614 (W). Thurston Co.: Gate, 1912, Foster 2011 (W).

7. Caloplaca elegans (Link) Th. Fr. Lich. Scand. $1: 168.1871-74$.

Lichen elegans Link, Ann. der Naturg. 1:37. 1791.

Placodium elegans (Link) DC.

Thallus flame-scarlet $(R)$, ochraceous-orange ( $R$ ), or yellow, foliose or squamulose, radiate-stellate or orbicular, appressed, thick, lobes linear, much branched, knotted and twisted, surface slightly pitted or lacunose, margins often lobed. Apothecia $0.4-2.0 \mathrm{~mm}$. in diameter, subsessile to sessile; disk concolorous with the thallus, concave to slightly convex; thalline margin slightly paler than the disk, entire or slightly crenulate; spores hyaline, ovoid-ellipsoid, polar-bilocular, 9.0-16.0 × 5.0-9.0 $\mu$. Both epithecium and thallus turn a very bright red with $\mathrm{KOH}$.

On rocks: Okanogan Co.: near Twisp, 5000 ft., 1931, 1302. Chelan Co. : Zena, 800 ft., 1931, 1332. Yakima Co.: Boulder Cave, 3000 ft., 1932. Pierce Co.: Mt. Rainier, Camp Muir, 9000 ft., 1909, Comm. Kneeland, Foster (F). Ferry Co. : Republic, 1912, Foster 2363b (F).

S. Caloplaca lobulata (Flk.) Hellb. Bih. Kgl. Sven. 21 :67. 1896.

Lecanora lobulata Flk. in Sprgl. Neue Entd. $1: 219.1820$.

Thallus yellow, ochraceous-orange ( $R$ ), or rufous, verrucose, or subareolate, sometimes composed of minute, slightly convex lobules, effuse, thin, margin when present of short crenate lobules. Apothecia 0.2-0.8 mm. in diameter, sessile; disk concolorous with the thallus, flat; thalline margin 
thick, raised, entire or slightly crenulate; spores hyaline, ellipsoid, polarbilocular, $10.0-14.0 \times 5.0-7.0 \mu$. Both epithecium and thallus purple with $\mathrm{KOH}$.

On rocks: San Juan Co.: Brown Island, 1928, 2091, 1940, 3254.

9. Caloplaca murorum (Hoffm.) Th. Fr. Lich. Scand. 1:170. 1871-74.

Lichen murorum Hoffm. Enum. Lich. 63. 1784.

Placodium murorum (Hoffm.) DC.

Thallus bright yellow or ochraceous-orange, foliose or squamulose, orbicular, often areolate, and verrucose toward the center, definitely lobed at the margins, lobes rather short and somewhat branched, or the whole thallus may be verrucose or squamulose without a definite margin, closely attached to the substratum. Apothecia $0.5-1.0 \mathrm{~mm}$. in diameter, subsessile to sessile; disk concolorous with the thallus, concave to nearly flat; thalline margin crenulate; spores hyaline, ovoid-ellipsoid, polar-bilocular, 12.9-15.9 $\mathrm{x}$ 4.9-7.0 $\mu$. Both epithecium and thallus purple with $\mathrm{KOH}$.

On rocks: San Juan Co.: Turn Island, 1928, 2093. Yakima Co. : Deadhorse Hill, 4500 ft., 1931, 979; gulch near Naches, 1350 ft., 1931, 909; 1940, 3176. Chelan Co.: butte near Chelan, 200 ft., 1931, 1358. Douglas Co.: Lower Grand Coulee, 1100 ft., 1931, 1527. Garfield Co.: Pomeroy, $1860 \mathrm{ft} ., 1931,1572$.

Much of the yellow and bright orange color on rocks in the state is due to various species of Caloplaca. C. elegans and C. murorum are especially beautiful and showy lichens. Plate XI, D.

\section{TELOSCHISTACEAE}

Thallus foliose, dorsi-ventral, well developed plectenchymatous layer or longitudinal hyphae form upper and lower cortices, distinct or indistinct algal and medullary layers; or rarely fruticose, radial, differentiated into a plectenchymatous cortex, surrounding the algal and medullary layers, attached to the substratum by rhizoids or by a definite holdfast. Apothecia circular, scattered, marginal, or terminal, sessile; proper margin absent, thalline margin present; asci 8-spored; spores hyaline, polar-bilocular or 3-septate. Epithecium usually violet, purple, or some shade of red with $\mathrm{KOH}$.

Alga Protococcus.

66. Xanthoria Th. Fr. Nova Acta Reg. Soc. Sci. Upsal. ser. 3, 3 :66. 1861.

Thallus bright colored, usually yellow or orange, foliose, prostrate and appressed, or somewhat ascending, imbricately lobate, smooth or rough, sometimes fibrillose, dorsi-ventral, with a well developed plechtenchymatous cortex on both surfaces, indistinct algal layer and loosely interwoven medullary layer, attached to the substratum by rhizoids. Apothecia minute to medium sized, circular, scattered or marginal, sessile to subsessile; 
thalline margin present; hypothecium hyaline; paraphyses forked near the clavate tip, separate, septate; asci 8-spored; spores hyaline, polar-bilocular.

A. Thallus loosely attached, margins ascending, granulose-powdery.

B. Apothecia abundant; thallus small........ 3. X. candelaria var. pygmaea

B. Apothecia not abundant; thallus larger.

C. Lobes much lacero-dissected, bearing numerous fibrils.

2. $X$. candelaria var. laciniosa

C. Lobes slightly lacero-dissected, not bearing numerous fibrils, margins crenate.

1. X. candelaria

A. Thallus closely attached, margins not ascending, not granulose-powdery.

4. X. polycarpa

1. Xanthoria candelaria (L.) Kickx. Fl. Crypt. Flandres. 1 :229. 1867.

Lichen candelaria Linn. Sp. P1. 1141, 1753.

Teloschistes candelarius (L.) Fink.

Theloschistes lychneus (Ach.) Tuck.

Thallus apricot yellow $(R)$ or apricot orange $(R)$, foliose, medium sized, substellate or effuse, sometimes forming a thick powdery crust, usually composed of rather broad, deeply cut, branched lobes, which are more or less imbricated, appressed to somewhat ascending, margins granulose or powdery; below yellow to almost white, bearing concolorous rhizoids, and marginal fibrils. Apothecia rather rare, 0.5-3.0 mm. in diameter, subsessile to sessile; disk orange, concave to flat; thalline margin thin, crenate or granulose; spores hyaline, broadly ellipsoid, polar-bilocular, 11.0-16.0 x 6.6-8.0 $\mu$. Epithecium pinkish or purple with $\mathrm{KOH}$.

On trees, wood, and rocks : Pacific Co. : North Head, 211 ft., 1903, 458. San Juan Co.: Brown Island, 1940, 3196. Wahkiakum Co.: Cathlamet, 1940, 3349. Lewis Co.: Chehalis, 1930, 415. Kitsap Co.: Hidden Ranch near Bremerton, 213 ft., 1930, 531. King Co.: Edgewood, 300 ft., 1931, 1802. Kittitas Co.: Easton, $2168 \mathrm{ft} ., 1931$, 832. Yakima Co.: Rattlesnake Canyon, 2000 ft., 1931, 1532. Klickitat Co.: Goldendale, 2000 ft., 1931, 1685a. Chelan Co.: butte near Chelan, 2000 ft., 1931, 1331. Ferry Co. : Mt. Gibraltar, 3100 ft., 1940, 3152. Garfield Co.: Pomeroy, 1500 ft., 1940, 2739. San Juan Co.: San Juan Island, Friday Harbor, 1905, Frye (W). Island Co.: Whidbey Island, Langley, 1924, J. M. Grant (F). Ferry Co. : Republic, 1912, Foster 2323 (F). Walla Walla Co.: Wallula, 1906, Foster 299 (F). Spokane Co. : Spokane, 1906, T. A. Bonser 18 (F).

A common lichen widely distributed over the state.

2. Xanthoria candelaria var. laciniosa (Duf.) Arn. Flora $67: 244.1884$. Parmelia parietina var. lacinosa Duf. in Schaer. Enum. Critic. Lich. Eur. 51. 1850.

Teloschistes candelaria var. laciniosus (Duf.) Fink.

Theloschistes lychneus f. laciniosa Tuck. 
Lobes of the thallus much lacero-dissected, margins ascending, often bearing numerous fibrils; otherwise like the species.

On trees and rocks: San Juan Co.: Brown Island, 1931, 2095. Yakima Co. : Deadhorse Hill, 4000 ft., 1931, 958.

3. Xanthoria candelaria var. pygmaea (Bory) Arn. Flora $67: 244.1884$.

Parmelia parietina var. pygmaea Bory in Fries. Lich. Eur. Reform. 73. 1831.

Theloschistes lychneus var. pygmaeus (Bory) Tuck.

Teloschistes candelarius var. pygmaeus (Bory) Fink.

Thallus very small, suborbicular, somewhat cushion like, lobes ascending. Apothecia often numerous; otherwise like the species.

On trees and rocks: Wahkiakum Co.: Cathlamet, 1940, 2907. Kitsap Co.: Hidden Ranch, near Bremerton, 213 ft., 1930, 1889. King Co.: Woodinville, 1940, 3349. King Co. : Edgewood, 300 ft., 1940, 3084. Thurston Co.: Grand Mound, 1940, 2952. Grant Co.: Blackrock Lake, 1929, G. N. Jones 3049 (W).

4. Xanthoria polycarpa (Hoffm.) Oliv. Rev. de Bot. 12: 96, 1894.

Lobaria polycarpa Hoffm. Deutschl. Fl. 2: 159. 1795.

Theloschistes polycarpus (Ehrh.) Tuck.

Thallus apricot yellow ( $R$ ) or apricot orange $(R)$, foliose, small to medium sized, suborbicular to irregular, consisting of many small, narrow, much divided lobes, closely adnate or rarely ascending, margins smooth, crenate; below pale yellow or grayish white, bearing concolorous rhizoids and marginal fibrils. Apothecia numerous, sometimes concealing the thallus, 1.0-3.0 $\mathrm{mm}$. in diameter, subsessile to sessile; disk deep orange, concave to flat; thalline margin thin, crenulate, spores hyaline, ellipsoid, polar-bilocular, 11.0-16.0 $\times 6.0-8.0 \mu$. Epithecium pinkish or purple with $\mathrm{KOH}$.

On trees and old wood: San Juan Co.: San Juan Island, English Camp, 1928, 2096. King Co. : Edgewood, 300 ft., 1931, 1824. Kittitas Co. : Easton, 2168 ft., 1931, 839. Chelan Co.: Wenatchee Lake, 1800 ft., 1931, 1067. Douglas Co.: Upper Grand Coulee, 1550 ft., 1931, 1514. Whitman Co.: Tekoa Mountains, 2900 ft., 1931, 1591. Wahkiakum Co.: Cathlamet, 1907, Foster 487 (F). Clallam Co.: Sequim, J. M. Grant 199 (F). Thurston Co.: Tenino, 1904, Frye 14 (F) ; Hamilton 1905, Foster (F). Whitman Co.: Pullman, 1900, R. K. Beattie (F).

The numerous apothecia make this rather an easy Xanthoria to identify. It is widely distributed over the state.

\section{BUELLIACEAE}

Thallus crustose or rarely squamulose, entire or radiately lobed at the margins, dorsi-ventral, little or no differentiation into layers, attached to the substratum by hyphal rhizoids. Apothecia circular, scattered on the surface of thallus, imbedded to adnate or sessile; either proper or thalline 
margin well developed, rarely both margins well developed and persistent; asci 1-8-spored, rarely 16-24-spored; spores smoke gray ( $R$ ), brown or black, 1-3-septate, or sometimes muriform.

Alga Protococcus.

A. Apothecia with prominent proper margin (lecideine) ..........67. Buellia A. Apothecia with prominent thalline margin (lecanorine)....68. Rinodina

67. Buellia De. Not. Gior. Bot. Ital. 2:195. 1846.

Thallus crustose or squamulose, uniform or marginally lobed, usually without either cortex, if present only a rudimentary one. Apothecia circular, scattered, immersed to sessile; only proper margin present, soon disappearing; hypothecium brown or black; paraphyses simple, or rarely branched, usually enlarged at the tip; asci 1-8-spored; spores brown or black, 1-3-septate or muriform, septa thick, without a mucilaginous halo.

A. Thallus dark colored.

B. Thallus squamulose.

10. B. badia

B. Thallus not squamulose.

C. Thallus olive-gray, forming a thin fissured crust.

3. B. punctata

C. Thallus not olive-gray, forming a thick folded crust.

5. B. turgescens

A. Thallus light colored.

D. On rocks.

E. Thallus with a powdery appearance, disk usually white-

pruinose, spores muriform when mature...............6. B. alboatra

E. Thallus without a powdery appearance, disk not white-

pruinose, spores 1 -septate.

4. B. stellulata

D. On trees, old wood, over moss or thin layer of soil.

F. Spores always 1 -septate.

1. B. disciformis

F. Spores not always 1-septate.

$\mathrm{G}$. Spores muriform when mature.

H. Black hypothallus often conspicuous; asci usually less than 8-spored, spores large, usually more than $28 \mu$ long. 8. B. oidalea

H. Black hypothallus not often conspicuous; asci usually 8 -spored, spores less than $28 \mu$ long.

I. Disk white-pruinose.

6. B. alboatra

I. Disk not white-pruinose.

9. B. penichra

G. Spores not muriform when mature.

J. On mosses or soil, spores usually 1-septate.

2. B. papillata

J. On trees or old wood, spores usually 3-4-locular.

7. B. lauri-cassiae

1. Buellia disciformis (Fries) Mudd, Manual Brit. Lich. 216. 1861.

Lecidea parasema var. disciformis Fries Nov. Sched. Critic. 9. 1826. Buellia parasema (Ach.) De Not. 
Thallus olive-gray (R), dark gray, grayish white, or slightly yellow, crustose, when young, thin and smooth, but soon becoming thicker and rough, granulate, chinky and at length areolate, black hypothallus not often visible. Apothecia $0.4-0.8 \mathrm{~mm}$. in dianeter, adnate to sessile or rarely slightly immersed; disk black or sometimes brownish black, flat to convex, finally turgid, the thin, entire, or flexuous proper margin rarely disappearing; hypothecium brownish black; paraphyses slender coherent, asci 8-spored; spores brown, oblong-ellipsoid, 1-septate, 16.0-24.0 × 7.5-11.0 $\mu$.

On trees, old wood, moss or thin layer of soil: Pacific Co.: Chinook, 1931, 800; Long Beach, 1940, 2761. Island Co.: Whidbey Island, 1931, 1768. Kittitas Co.: South Cle Elum, 3000 ft., 1931, 880. Yakima Co.: Rattlesnake Canyon, 2000 ft., 1931, 1537. Grays Harbor Co. : Pacific Beach, 1910, Foster 1418 (F). Thurston Co.: Gate, 1912, Foster 1988 (W). King Co.: Seattle, University of Washington campus, 1904, Frye (W). Ferry Co.: Republic, 1912, Foster 2299 (F).

A common Buellia, widely distributed in the state.

2. Buellia papillata (Somrft.) Tuck. Lich. Calif. 26. 1866.

Lecidea papillata Somrft. Suppl. Fl. Lapp. 154. 1826.

Buelliopsis papillata (Somrft.) Fink.

Thallus white or grayish white, crustose, minutely granulose, forming a thin to finally thick crust, becoming rough and warty, often crumbling. Apothecia 0.3-1.0 mm. in diameter, adnate, often clustered and confluent; disk black, flat to convex, tuberculate; proper margin thin and soon disappearing; hypothecium brown; paraphyses distant; asci 8-spored; spores brown, ellipsoid or subfusiform, 1-3-septate, usually 1-septate, 19.8-30.0 x 9.6-12.0 $\mu$.

On mosses and soil: Yakima Co.: Deadhorse Hill, 4000 ft., 1931, 567. Lincoln Co.: Davenport, $2450 \mathrm{ft} ., 1931,1506$. Olympic Mountains, Steeple Peak, 5500 ft., A. H. Sinith 2325 (M). Ferry Co.: Republic, 1912, Foster 2299 (F).

3. Buellia punctata (Hoffm.) Mass. Ricerch. Auton. Lich. 81. 1852.

Verrucaria punctata Hoffm. Deutschl. F1. 192. 1796.

Buellia myriocarpa (DC.) De Not.

Thallus olive-gray $(R)$ or olive-brown $(R)$, crustose, thin, scurfy, finally forming a rough warty, fissured crust, at times disappearing entirely. Apothecia 0.2-0.5 mm. in diameter, adnate; disk black, flat to convex; proper margin paler than the disk, thin and disappearing; hypothecium dark brown; paraphyses free, rather slender, tips ellipsoid or oblong; asci 8-spored; spores brown, ellipsoid, 1-septate, 10.0-16.4 x 4.0-6.1 $\mu$.

On trees, old wood, and rocks: San Juan Co.: Turn Island, 1928, 2098; Brown Island, 1906, Fink 59 (W). Washington Territory, 1882, Suksdorf. 198 (S). 
4. Buellia stellulata (Tayl.) Mudd, Man. Brit. Lich. 216. 1861.

Lecidea stellulata Tayl. in Mack. Fl. Hibernica 2:118. 1836.

Thallus white, grayish-white, or gray, crustose, composed of minute, flat to slightly convex areoles, scattered or crowded upon a black hypothallus. Apothecia 0.1-0.2 $\mathrm{mm}$. in diameter, immersed to adnate; disk black, flat to somewhat convex; proper margin black or sometimes slightly gray, thin, long persistent but finally disappearing; hypothecium dark brown; paraphyses subcoherent, thread-like, tips rounded; asci 8-spored; spores brown, ellipsoid, 1-septate, 7.0-9.9 x 4.0-6.1 $\mu$.

On rocks: San Juan Co. : San Juan Island, 1928, 1947.

5. Buellia turgescens (Nyl.) Tuck. Gen. Lich. 185. 1872.

Lecidea turgescens Nyl. Mem. Soc. Sci. Nat. Cherbourg 5 :337. 1857.

Thallus brown, olive-brown ( $\mathrm{R}$ ), or slightly rufescent, crustose, areolate, chinky, warty, at length forming a somewhat folded crust. Apothecia 0.2-0.8 $\mathrm{mm}$. in diameter, immersed to adnate; disk black, flat to convex; proper margin black, thin, sometimes disappearing; hypothecium dark brown; paraphyses free, slender, tips ellipsoid; asci 8-spored; spores brown, ellipsoid, 1-septate, 9.0-16.0 x 4.0-7.0 $\mu$.

On old wood: San Juan Co.: San Juan Island, 1906, Fink 449 (W).

6. Buellia alboatra (Hoffm.) Brantl. and Rostr. Bot. Tidsskrift. 4. 239. 1869.

Lichen alboater Hoffm. Enum. Lich. 30. 1784.

Rhizocarpon alboatrum (Hoffm.) Anzi.

Thallus white or pale grayish white, crustose, thin, at times thicker, smooth to slightly warty, often with a powdery appearance. Apothecia 0.3-1.0 $\mathrm{mm}$. in diameter, immersed to adnate; disk black, often whitepruinose, flat to convex; proper margin black, thin, at length disappearing; hypothecium brown; paraphyses becoming distant, with slightly enlarged brown tips; asci 8-spored; spores brown, oblong-ellipsoid, muriform, 10.0 $24.0 \times 5.0-9.0 \mu$.

On trees or old wood: Clallam Co.: Elwha River, 244 ft., 1940, 2875. San Juan Co.: Waldron Island, 1906, Fink 251 (W). Herre (1917) reports it from Whatcom Co.: "common on trunks of trees, especially Douglas spruce."

7. Buellia lauri-cassiae (Fee) Müll. Arg. Rev. Mycol. 9:85. 1887.

Lecidea lauri-cassiae Fee Suppl. Essai Cryptog. Ecorc. Officin. 101. 1837.

Buellia parasema var. triphragmia (Nyl.) Th. Fr.

Thallus olive-gray ( $R)$, dark gray, grayish-white, or yellow-white, crustose when young, thin and smooth, but soon becoming thicker and rough, chinky and at length areolate, black hypothallus not often visible, 
the thallus is very similar to that of $B$. disciformis (Fries) Mudd. Apothecia 1.0-2.0 $\mathrm{mm}$. in diameter, adnate to sessile, rarely almost immersed; disk black, flat to slightly convex; proper margin black, thin, entire to flexuous, rarely disappearing; hypothecium dark brown; paraphyses coherent, tips brown; asci 8-spored; spores brown, oblong-ellipsoid, when mature 2-3septate, $19.0-24.6 \times 8.2-11.0 \mu$.

On trees or dead wood: San Juan Co.: San Juan Island, Pt. Caution, 1928, 1986. Fink (1935) reports this species collected in Washington.

8. Buellia oidalea (Nyl.) Tuck. Lich. Calif. 26. 1866.

Lecidea oidalea Nyl. Memoir. Soc. Imp. Sci. Nat. Cher. 5:127. 1857.

Rhizocarpon oidaleum (Tuck.) Fink.

Thallus olive-buff $(R)$, olive-gray $(R)$, or grayish-white to almost white, crustose, thin and smooth or thick, rough and warty, more or less limited by the black hypothallus. Apothecia $0.5-1.5 \mathrm{~mm}$. in diameter, sessile; disk dull black, flat to convex; proper margin thick, entire, soon disappearing; hypothecium brown; paraphyses loosely coherent, threadlike, usually simple, some forked below the slightly enlarged tips; asci 1- or 2-6-spored, rarely 8-spored; spores brown, oblong-ellipsoid, muriform, $28.0-40.4 \times 14.0-17.0 \mu$.

On trees: Clallam Co.: Lake Sutherland, $590 \mathrm{ft} .$, 1931, 1028. San Juan Co.: Orcas Island, Mt. Constitution, 2500 ft., 1928, 2099. Island Co.: Whidbey Island, 1931, 1769. Kitsap Co.: Hidden Ranch near Bremerton, 213 ft., 1930, 1934. Clallam Co.: Port Angeles, 1914, Foster 2623 (F); Sequim, 1916, J. M. Grant 808 (F). Thurston Co.: Gate, 1912, Foster 1981 (F). King Co. : Ronald Bog, 1912, Foster 2080 (F).

9. Buellia penichra (Tuck.) Hasse Contri. U.S. Nat. Herb. 17:122. 1913. Buellia oidalea var. penichra Tuck. Syn. N. Am. Lich. 2:99. 1888.

Rhizocarpon penichrum (Tuck.) Merrill.

Thallus white, grayish-white, or greenish-gray, crustose, smooth or slightly rough, becoming somewhat areolate, occurring in small patches, black hypothallus not always visible. Apothecia $0.3-0.8 \mathrm{~mm}$. in diameter, sessile; disk dull black, flat to slightly convex; proper margin turgid, glistening, slightly raised, finally disappearing; hypothecium yellowish-brown; paraphyses loosely coherent; asci 6-8-spored; spores brown, oblong-ellipsoid, muriform, never more than 5 transverse septa, 19.8-23.1 x 9.9-10.6 $\mu$.

On trees : Kittitas Co. : Lake Cle Elum, $2200 \mathrm{ft}$., 1931, 886. Chelan Co. : Rainbow Trail, 3500 ft., 1931, 1267. Columbia Co. : Blue Mountains, 3500 ft., 1629. Ferry Co.: Republic, 1912, Foster 2315 (W). Washington Territory, Suksdorf (F).

10. Buellia badia (Fries) Mass. Mem. Lichenogr. 124. 1853.

Lecidea badia Fries Syst. Orb. Veg. 287. 1825. 
Thallus dark olive-brown ( $R$ ) or greenish-brown, squamulose, composed of flat or rounded crenately lobed squamules or the thallus may become areolate. Apothecia 0.2-1.0 mm. in diameter, adnate; disk black, flat to convex; proper margin thin, raised, black, becoming irregular and finally disappearing; hypothecium brown; paraphyses distinct, slender, with brown globose tips; asci 8 -spored; spores brown, ellipsoid, 1-septate, $10.0-15.0 \times 6.0-7.0 \mu$.

On moss growing over rock: Washington Territory, Suksdorf, 112, 274 (F). Fink (1935) reports this species from Washington.

One must have material bearing mature spores to make correct determinations of Buellia species.

\section{Rinodina Ach. S. F. Gray, Nat. Arr. Brit. P1. 1 :448. 1821.}

Thallus crustose, seldom squamulose, uniform all over or sometimes marginally lobed, cortex is usually poorly developed, except in the squamulose forms, where it becomes plectenchymatous, a thin algal layer and a more or less well developed medullary layer of loosely interwoven hyphae are always present. Apothecia circular, immersed to adnate or sessile, proper margin very thin, often lacking; with thalline margin usually present, concolorous with the thallus, rarely disappearing; hypothecium hyaline to brownish; paraphyses rarely branched, thread-like, often thickened at the tips; asci usually 8-spored, rarely as many as 24-spored; smoky gray, brown, or black, 1 to rarely 3 -septate, with strong thickened walls, polar-bilocular cell contents usually united by an isthmus.

A. On bark.

B. Hypothallus conspicuous, apothecia reddish brown.

3. R. Hallii

B. Hypothallus not conspicuous, apothecia brown or black.

5. $R$. sophodes

A. Not on bark.

C. On soil.

D. Spores 1-septate.

4. R. orbata

D. Spores becoming 3-septate.

2. R. Conradi

C. Not on soil.

E. Thallus lobed at periphery, on rock.

6. R. oreina

$E$. Thallus not lobed at periphery, on rock, rarely on wood.

1. R. confragosa

1. Rinodina confragosa (Ach.) Körb. Syst. Lich. German 125. 1855.

Parmelia confragosa Ach. Meth. Lich. Suppl. 33. 1803.

Rinodina sophodes var. confragosa (Ach.) Tuck.

Thallus white or grayish-white, crustose, thin or rather thick, coarsely granulose or becoming warty, granules scattered upon an inconspicuous black hypothallus, which is sometimes not visible at all. Apothecia 0.5-1.3 $\mathrm{mm}$. in diameter, adnate to sessile; disk brownish-black to black, flat; 
thalline margin thick, crenulate, somewhat wavy, often inflexed; hypothecium pale yellowish; spores brown, ovoid-ellipsoid, 1-septate, polar-bilocular, $18.0-27.1 \times 8.0-15.0 \mu$.

On rocks, more rarely on trees, or old wood: San Juan Co.: Turn Island, 1928, 2100.

2. Rinodina Conradi Körb. Syst. Lich. Ger. 123. 1855.

Thallus greenish-gray or brownish-gray, crustose, minutely granulose, granules flat or slightly convex, scattered or forming a thin chinky crust. Apothecia 0.3-0.8 mm. in diameter, sessile; disk flat to convex, blackishbrown or black; thalline margin thick, almost entire or crenulate; hypothecium yellowish; spores brown, ellipsoid, 1-3-septate transversely, and rarely the middle cell 1 -septate longitudinally, polar-bilocular, 26.4-31.3 x 11.0-16.5 $\mu$.

On moss, rocks, or on a thin layer of soil over rocks: Ferry Co.: Republic, 1912, Foster (F).

\section{Rinodina Hallii Tuck. Bull. Torr. Bot Club 5 :20. 1874 .}

Thallus smoke-gray ( $R$ ), grayish-brown. or pale green-brown, crustose, thin, chinky or areolate, bounded by a conspicuous broad, black hypothallus. Apothecia numerous, 0.7-1.2 mm. in diameter, adnate; disk reddishbrown or black, when young slightly pruinose; the thalline margin thin, concolorous with the thallus, sometimes excluded; hypothecium hyaline, or with a brownish tinge; spores brown, ellipsoid or oblong-ellipsoid, 1-septate, polar-bilocular, $19.8-30.0 \times 6.8-10.0 \mu$.

On trees: Pacific Co.: Loomis Lake, 1931, 735. Grays Harbor Co.: Copalis, 1911, Foster 1632 (W) ; Westport, 1907, Foster 566 (718) (F). Washington Territory, Suksdorf 295 (F).

4. Rinodina orbata (Ach.) Wainio Arkiv. för Bot. 8:71. 1909.

Lecanora sophodes var. orbata Ach. Lichenogr. Univers. 678. 1810.

Rinodina turfacea (Wahl.) Körb.

Thallus brownish-ash colored or fuscous ( $R$ ), crustose, verrucose, or granulate, encrusting the substratum. Apothecia small, $0.5-1.0 \mathrm{~mm}$. in diameter, adnate to subsessile; disk reddish-brown to brownish-black, flat to convex, dull; thalline margin thin, raised, entire or rugulose, persistent; hypothecium pale yellowish; spores brown, oblong-ellipsoid, 1-septate, polar-bilocular, $20.0-28.0 \times 12.0-16.0 \mu$.

On soil or mosses: Whatcom Co.: Mt. Baker, 6000 ft., Herre (1917). Fink (1935) reports this species from Washington. No Washington specimen seen by the writer.

5. Rinodina sophodes (Ach.) Mass. Ricerch. Auton. Lich. 14. 1852.

I.ichen sophodes Ach. Lichenogr. Sinec. Prodro. 67. 1798. 
Thallus gray, brownish-olive, or olive-brown ( $R$ ), crustose, thin, rough, granulate-areolate, continuous or scattered on a thin black hypothallus. Apothecia 0.3-0.8 mm. in diameter, adnate; disk brown or brownish black, flat to convex; with thin black proper margin, and prominent lighter colored crenulate thalline margin, rarely disappearing; hypothecium hyaline or pale brown; spores brown, oblong-ellipsoid, 1-septate, polar-bilocular, 19.8-23.0 x 6.6-9.0 $\mu$.

On rock, trees, or old wood: Spokane Co.: Mt. Spokane, 5808 ft., 1931, 1490. San Juan Co.: San Juan Island, Roche Harbor, 1906, Fink 289 (W). Washington Territory, Suksdorf (F).

6. Rinodina oreina (Ach.) Mass. Ricerch. Auton. Lich. 16. 1852.

Lecanora straminea var. oreina Ach. Lichenogr. Univers. 433. 1810.

Thallus deep olive-buff $(R)$ or straw yellow ( $R$ ), crustose becoming squamulose, thin or rather thick, very closely attached to the substratum, verrucose-areolate, areoles small, passing into rather linear, branched, crowded, black-edged squamules at the circumference, often blackish-suffused, giving the whole plant a blackish appearance. Apothecia 0.3-0.8 mm. in diameter, immersed to adnate, or subsessile; disk black, turgid, flat to slightly convex; thalline margin obtuse, concolorous with the thallus, rarely disappearing; hypothecium hyaline; spores brown, ellipsoid, 1-septate, polar-bilocular, 9.0-12.0 × 5.0-7.0 $\mu$.

On rock: Ferry Co.: Republic, 1912, Foster 2381 (F). No location given, 1913, J. S. Weaver (F).

For the correct determination of most Rinodina species it is necessary to have good mature fruiting specimens.

\section{PHYSCIACEAE}

Thallus foliose, rarely fruticose, usually circular, appressed or ascending, dorsi-ventral or radial, differentiated into well developed plectenchymatous cortices, medullary and algal layers, attached to the substratum by rhizoids. Apothecia circular, adnate to sessile; with either a proper or a thalline margin or both, asci 8-spored, spores brown, 1-septate, rarely 3-septate, or muriform, thick cell well.

Alga Protococcus.

69. Physcia Kgl. Vet. Akad. Nya Handl. 15 :252. 1794.

Thallus foliose, more or less lobed often sorediate, usually circular in shape, appressed or ascending, upper and lower cortices of vertical extending hyphae, definite algal and medullary layers, medulla cottony, white, saffron yellow, or red; attached to the substratum by rhizoids. Apothecia round, minute to large, adnate to sessile; thalline margin concolorous with the thallus; hypothecium hyaline or dark; paraphyses unbranched, septate, or rarely nonseptate; asci normally 8-spored; spores brown, oblong or ellipsoid, usually 1-septate, rarely muriform, cell wall thick. 
A. Thallus definitely brown or more rarely grayish brown...5. P. muscigena

A. Thallus never brown or grayish brown.

B. Thallus sorediate.

C. Soredia on margins of thallus.

D. Lower surface of thallus dark brown or black, upper cortex shows no color change with $\mathrm{KOH}$.

4. P. leucoleiptes

$D$. Lower surface of thallus white or light buff, upper cortex yellow with $\mathrm{KOH}$.

8. P. tribacia

C. Soredia scattered over surface of thallus, upper cortex yellow with $\mathrm{KOH}$. 2. P. caesia

C. Soredia on the under surface of inflated vaulted tips, upper cortex shows no color change with $\mathrm{KOH}$.

B. Thallus never sorediate.

3. P. hispida

E. Thallus pruinose, always light colored, margins of lobes prostrate.

E. Thallus not pruinose.

6. $P$. pulverulenta

F. Lower surface of thallus white, with concolorous rhizoids.

F. Lower surface of thallus brown or black, with concolorous rhizoids. 1. P. aipolia

1. Physcia aipolia (Ehrh.) Hampe in Fürnr. Nat. Topogr. Regensburg, $2: 249.1839$.

Lichen aipolius Ehrh. in Humb. Fl. Friburg. Specim. 19. 1793.

Physcia stellaris var. aipolia (Ehrh.) Hampe.

Thallus pale grayish-green or drab $(R)$, foliose, circular, expanded, appressed, lobes much divided, narrow elongated, sinuous, imbricated, margins entire or crenate; below brown or black, with concolorous rhizoids. Apothecia 0.5-1.5 mm. in diameter, sessile; disk dark brown or black, white pruinose, concave to slightly convex, thalline margin thick, prominent concolorous with the thallus, somewhat crenate; hypothecium hyaline; spores brown, ellipsoid, 1-septate, 16.8-25.0 x 6.9-11.0 $\mu$. Cortex and medulla yellow with $\mathrm{KOH}$.

On trees and rocks: Yakima Co.: American River Camp, $2800 \mathrm{ft.}$, 1931, 949. Clallam Co.: Elwha River, near Port Angeles, 1940, 2930. Clallam Co.: Dungeness, 1913, Foster 2543 (F).

2. Physcia caesia (Hoffm.) Hampe in Fürnr. Nat. Topogr. Regensburg, $2: 250.1839$.

Lichen caesius Hoffm. Enum. Lich. 65. 1788.

Thallus pale greenish gray or ivory yellow ( $R$ ), foliose, circular, adnate, lobes branched, narrow elongated, somewhat imbricated, bearing circular spots of gray soredia, margins entire or crenate; below pale or rarely black, with black rhizoids. Apothecia 0.5-2.0 mm. in diameter, sessile; disk dark brown or black, often gray pruinose, slightly concave to flat; thalline margin inflexed, entire or crenulate; hypothecium hyaline; spores brown, ellipsoid, 1-septate, 19.8-22.0 × 6.6-12.0 $\mu$. Cortex yellow with $\mathrm{KOH}$. 
Usually on rocks, rarely on old wood or trees: Pacific Co.: Long Beach, 1940, 2758. Clallam Co. : Elwha River Bridge, 1940, 2873. Chelan Co.: Beaver Summit, 2800 ft., 1934, 2474. San Juan Co.: Brown Island, $1940,3195$.

This species is easily recognized by the circular masses of soredia scattered over the upper surface of the thallus.

\section{Physcia hispida (Schreb.) Frege, Deutsch. Bot. Tasch. 169. 1812.}

Lichen hispidus Schreb. Spicil. Fl. Lipsiens. 126. 1771.

Thallus pale greenish gray, or ivory yellow (R), silvery gray or greenish gray, foliose, often growing in small circular clusters, loosely adnate or sub-erect, lobes stellately arranged, narrow palmately divided, distinct and separate or imbricated, soredia borne on under surface of inflated, vaulted tips, margins entire or crenate, often ciliate; below white, mealy, bearing numerous either light or dark rhizoids. Apothecia 0.5-2.0 mm. in diameter, sessile to subsessile; disk brown or black, usually pruinose, slightly concave to flat; thalline margin entire to crenate; hypothecium hyaline; spores brown, ovoid-ellipsoid, 1 -septate, $15.0-22.0 \times 6.0-10.0 \mu$. Cortex shows no color change with $\mathrm{KOH}$.

On trees and rocks: Pacific Co.: Long Beach, 1940, 2758. San Juan Co.: San Juan Island, Pt. Caution, 1931, 2101. Grays Harbor Co.: Westport, 1908, Foster 665 (W). San Juan Co.: San Juan Island, Friday Harbor, 1905, Frye 22 (F). Clallam Co.: Dungeness, 1914, Foster 2526 (W) ; Sequim, 1916, J. M. Grant 604 (F). Island Co.: Whidbey Island, Langley, 1924, J. M. Grant $213 e$ (F).

This species is rather common on trees close to the salt water. It is easily distinguished by the numerous sorediate vaulted tips.

4. Physcia leucoleiptes (Tuck.) Lettau in Hedw. 52:254. 1912.

Physcia pulverulenta var. leucoleiptes Tuck. Proc. Am. Acad. Arts and Sci. $1: 224.1848$.

Physcia pulverulenta (Schreb.) Nyl.

Thallus white or pale drab-gray, foliose, pruinose, circular, appressed, lobes long, branched, somewhat imbricated, margins entire or crenate, interruptedly elevated, bearing white or yellow soredia; below dark brown or black. Apothecia rare, no fruiting material collected in the state has been seen by the writer, the following description is from a Tuckerman specimen collected, Cambridge, Mass., in 1837 (F), apothecia $2.0-4.0 \mathrm{~mm}$. in diameter, sessile to subsessile; disk pruinose, concave to flat; thalline margin swollen, sorediate; hypothecium pale brown; spores brown, 1-septate, $23.0-35.0 \times 18.0-27.0 \mu$. Cortex shows no color change with $\mathrm{KOH}$.

On trees or on thin layer of soil over rock: Douglas Co. : Lower Grand Coulee, 1100 ft., 1931, 1524. Chelan Co.: Beaver Summit, 2800 ft., 1931, $1334 a$. 
This species is easily separated from Physcia pulverulenta by the dark under surface and the abundantly sorediate margin.

5. Physcia muscigena (Ach.) Nyl. Act. Soc. Linn. Bordeaux 21:308. 1856.

Parmelia muscigena Ach. Lichenogr. Univers. 472. 1810.

Physcia pulverulenta var. muscigena (Ach.) Nyl.

Thallus grayish brown or brown, foliose, spreading irregularly, lobes divided, short and broad, tips widened like a fan, margins crenate, ascending, pruinose over the whole surface or only at the tips, margins sometimes sorediate, medulla yellow, green, or white; below dark brown or black, paler near the circumference, covered with black rhizoids; loosely attached to the substratum. Apothecia small and rare, no Washington material seen fruiting by the writer, apothecia $1.0-2.0 \mathrm{~mm}$. in diameter, sessile; disk black, more or less pruinose, concave to flat, thalline margin thick, persistent, entire or crenate, becoming sorediate, hypothecium pale brown; spores brown, ellipsoid, 1-septate, 22.0-26.4 x 10.0-14.8 $\mu$. This description was made from a specimen in Hasse Herbarium No. 188, collected Ventura Co., Calif. (S).

On trees, moss, or a thin layer of soil over rock: Yakima Co.: near Naches Ranger Station, 2400 ft., 1940, 3327; gulch near Naches, 1350 ft., 1931, 905. Ferry Co. : near Republic, 2400 ft., 1940, 3140. Olympic Mountains, 6000 ft., 1915, J. M. Grant (F). Ferry Co.: Republic, 1912, Foster 2360 (F). San Juan Co.: Waldron Island, 1906, Fink 255 (W), this specimen determined as Physcia pulverulenta by Fink.

The dark color of the thallus separates this species from any other, species of Physcia.

6. Physcia pulverulenta (Schreb.) Hampe in Fürnr. Nat. Topogr. Regensburg, $2: 249$. 1839.

Lichen pulverulentus Schreb. Spicil. Fl. Lipsiens. 128. 1771.

Thallus pearl gray $(R)$, pale drab-gray $(R)$, or almost white, foliose, circular, closely appressed, usually white pruinose, lobes much divided, flat, smooth, often elongated, narrow, imbricated, tips wider, rounder, truncate or emarginate, margins entire, crenate or rarely lobulate, sometimes ascending, the central part of the thallus is often sparingly to abundantly covered with narrow secondary branches, medulla white; below brownish black or black, paler toward the margins, bearing numerous brown or black rhizoids. Apothecia rare, 1.0-4.5 mm. in diameter, sessile; disk dark brown or dark gray, white pruinose, concave or flat ; thalline margin thick, persistent, crenate or lobulate; hypothecium pale brown; spores brown, oblong-ellipsoid, 1-septate, $23.0-34.5 \times 14.0-21.0 \mu$.

On trees, old wood, rocks, moss, and soil : Chelan Co. : Beaver Summit, 2800 ft., 1931, 1334; Entiat, 800 ft., 1931, 1108. Yakima Co.: Naches Ranger Station, 2100 ft., 1940, 3323. Klickitat Co.: Goldendale, 2000 ft., 
1931, 1684. Columbia Co. : New Hope, 1500 ft., 1931, 1566; Dayton, 1700 ft., 1940, 2729. Klickitat Co.: Goldendale, 1909, Foster 1155 (W).

A common species east of the Cascades. It is abundantly white pruinose.

7. Physcia stellaris (L.) Nyl. Act. Soc. Linn. Bordeaux 21 :307. 1856.

Lichen stellaris Linn. Sp. Pl. 1144. 1753.

Thallus white, pearl gray ( $R$ ), or grayish olive ( $R$ ), foliose, orbiculatestellate, appressed, lobes narrow, usually elongated, much divided, rather convex, growing close together, but usually distinct, slightly pruinose, toward the tips, margin entire or crenate, surface smooth at the tips but rugose toward the center, often breaking up into a crust in the center, medulla white; below white or pale gray with concolorous rhizoids. Apothecia numerous, $1.0-5.0 \mathrm{~mm}$. in diameter, round or slightly angular, sessile; disk dark brown or black, usually pruinose, concave to convex; thalline margin thick, persistent, entire or crenate; hypothecium hyaline;

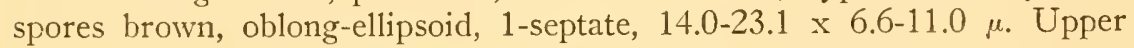
cortex yellow with $\mathrm{KOH}$.

On trees and rocks: Pacific Co.: Long Beach, 1940, 2757. Chelan Co.: Merritt, 2186 ft., 1931, 1070; Rainbow Trail, 3500 ft., 1931, 1259. Yakima Co. : Satus Road, 1054 ft., 1931, 1674. Ferry Co. : Republic, 2650 ft., 1931, 1408. Clallam Co.: Sequim, 1916, J. M. Grant 835 (F); Port Angeles, 1914, Foster 2659 (W). Grays Harbor Co.: Westport, 1908, Foster 725 (W). Whitman Co.: Pullman, 1900, Beattie 26 (F).

A common lichen rather widely distributed over the state. It is easily distinguished from other species of Physcia by the abundant apothecia.

8. Physcia tribacia (Ach.) Nyl. Flora $57: 48.1874$.

Lecanora tribacia Ach. Lichenogr. Univers. 415. 1810.

Thallus white, grayish-white, or pearly gray ( $R$ ), foliose, somewhat orbicular, rather small but these small clusters are inclined to grow together, loosely adnate, lobes much divided, usually short, sometimes becoming long, branched, imbricated, with a strongly ascending, much dissected, granulose, finally sorediate margin, sometimes forming a crust in the center; below white becoming light buff ( $R$ ), with a few scattered concolorous rhizoids. Apothecia rare, $1.0-1.5 \mathrm{~mm}$. in diameter, sessile to subsessile; disk brownish black to black, concave to flat, usually densely pruinose, thalline margin entire or crenulate; hypothecium hyaline; spores brown, oblongellipsoid, 1-septate, 15.0-18.2 x 7.0-9.9 $\mu$. Upper cortex yellow with $\mathrm{KOH}$.

On trees and rocks: Chelan Co.: butte near Chelan, 2000 ft., 1931, 1350. Okanogan Co. : butte near Twisp, 1700 ft., 1931, 1461. 


\section{PARTIAL LIST OF LITERATURE CONSULTED}

Berry, E. C.

1941 A monograph of the genus Parmelia in North America, North of Mexico. Ann. Mo. Bot. Gard. 28:31-146.

Dodge, C. W.

1929 A synopsis of Stereocaulon with notes on some exotic species. Ann. Crypt. Exot. Tome II, Fasc., 2.

Evans, A. W.

1930 The Cladoniae of Connecticut. Trans. Conn. Acad. Arts and Sci. 30:357-510.

1938 Notes on the Cladoniae of Connecticut III. Rhodora 40:4-26.

FINK, B.

1910 The lichens of Minnesota. Contrib. U. S. Nat. Herb. 14 :1-269.

1919 Additions to lichen distribution in North America. Mycologia $11: 298-307$.

1935 Lichen flora of the United States. University Press, University of Michigan.

Freeman, O. W., and H. H. Martin

1942 The Pacific Northwest. New York.

FREY, E

1933 Cladoniaceae, Umbilicariaceae. Rabh. Crypt.-Flora Bd. 9, 4 Abt., 1 Hälfte, $1-424$.

HASSE, H. E.

1913 The lichen flora of southern California. Contrib. U.S. Nat. Herb. 17:1-132.

HEDRICK, J.

1942 Lichens in and near the Olympic National Park, Washington. Mich. Acad. Sci. Arts, and Letters $27: 45-56$.

HERRE, A. C.

1910 The lichen flora of the Santa Cruz Peninsula, California. Proc. Wash. Acad. Sci. $12: 27-269$.

1917 Preliminary notes on the lichens of Whatcom Co., Washington. Bryologist $20: 76-84$.

1943 Two new species of lichens and new records for Washington State and North Carolina. Proc. Biol. Soc. Wash. 56:17-20.

Howard, G. E.

1937 Preliminary report on the lichens of the state of Washington. Bryologist $40: 91-112$.

Howe, R. H. JR.

1911 American species of Alectoria occurring north of the 15th parallel. Mycologia $3: 106-150$.

1914 North American species of the genus Ramalina. Bryologist $17: 49-52$.

JACKSON, B. D.

1928 A glossary of botanic terms. Ed. 4. Philadelphia.

JoHnson, G. T.

1938 The taxonomic importance and phylogenetic significance of the cephalodia of Stereocaulon. Ann. Mo. Bot. Gard. 25 :729-762.

LINDAU, G.

1923. Die Flechten. Berlin.

Maerz, A., and M. R. Paul

1930 A dictionary of color. New York. 
Martin, R. J., and E. Corbin

1930 Climatic summary of the United States. The state of Washington. U.S.D.A. Weather Bureau.

Merrill, G. K.

1911 Lichen notes No. 19. Containing something of record and comment, and describing one new species. Bryologist $14: 36-38$.

Piper, C.

1906 Flora of the state of Washington. Contrib. U. S. Nat. Herb. 11:1-637.

RIDGEWAY, R.

1912 Color standards and nomenclature. Washington, D. C.

SANDSTEDE, $\mathrm{H}$.

1931 The Gattung Cladonia. Rabh. Crypt.-Flora Bd. 9, 4 Abt., 2 Hälfte, 1-531.

Sмith, A. L.

1918, 1926 A monograph of the British lichens. Ed. 2, Pts. I and II. British Museum. London.

SNELL, W. H.

1936 Three thousand mycological terms. Publ. 2. Providence, R. I.

TORREY, R. H.

1935 Paraphenylenediamine, a new color test for lichens. Torreya $35: 110-112$.

TUCKERMAN, E.

1882, 1888 A Synopsis of the North American Lichens. Pts. I and II. Boston.

WEBER, W. A.

1944 The Botanical collections of Wilhelm N. Suksdorf, 1850-1932. Res. Studies of the State College of Wash. 12:51-121.

Zahlbruckner, A.

1926 Lichenes. Bd. 8. Engler, A. and K. Prantl. Die natürlichen Pflanzenfamilien. Ed. 2. Leipzig.

1921-1940 Catalogus Lichenum Universalis. 10 vols. Leipzig. 


\section{GLOSSARY}

A. Used as a prefix; not; without.

Ab. Used as a prefix; from; away.

Accuminate. Tapering gradually to a point.

Acicular. Needle-like; needle-shaped, very slender.

Acute. Sharp at the end; less than a right angle.

Adnate. Firmly attached; adhering to any thing; touching broadly.

Agglutinate. Glued together.

Aggregate. Crowded close together.

Amorphous. Shapeless; without definite form; having no regular structure.

Amphithecium. A layer of tissue containing both algal cells and fungal hyphae, continuing upward from the hypothecium, and in some angiocarpic lichens almost surrounding the hymenium.

Angiocarpous. Having a fruit enclosed within a distinct covering; fruit-body globular opening only by a pore at the summit; having the hymenium enclosed by a perithecial wall, or by an amphithecium or by both; having the hymenium covered by the exciple or exciples.

Angular. Irregular in shape.

Apex. Summit ; end farthest from the base or point of attachment.

Apothecium. Open cup-shaped or saucershaped fruit-body; a structure in which spores are borne.

Appendage. That which is appended to something; applied especially to processes of any kind.

Appendiculate. Furnished with appendages; an apothecium subtended by the apex of a branch or with a lacinia extending beyond it.

Applanate. Flattened out; horizontally expanded.

Appressed. Closely flattened down.

Arachnoid. Cobweb-like, cobwebbed.

Arcuate. Curved like a bow.

Areolate. Marked out in little spaces.

Areole. Areola, a little space marked out on a surface, separated from other areoles by cracks.

Articulate. Jointed.

Ascocarp. A spore bearing structure, consisting of hymenium, epithecium, hypothecium, and exciple or exciples.

Ascospore. A spore developed in an ascus.

Ascus. Sac-like cell in which spores are developed.

Ascyphous. Podetia without scyphae.
Aseptate. Without cross-walls or septa.

Barbate. Bearded.

Base. End opposite the apex; portion nearest the point of attachment.

$\mathrm{Bi}$-. Used as a prefix, double, twice, two, twofold.

Biatorine. Biatoroid, apothecium bright colored, waxy, proper margin present, thalline margin absent.

Bifacial. Having two fronts, the opposite faces alike.

Biserial. In two rows.

Caespitose. Cespitose, growing in tufts, growing in low tufty patches.

Caesious. Bluish gray.

Clavate. Club-shaped; growing gradually thicker towards the top.

Canaliculate. Furrowed; fluted; channeled.

Cancellate. Latticed; coarsely reticulated.

Canescent. Covered with a hoary down.

Cartilaginous. Tough; brittle, breaking with a snap.

Capitate. With a minute knob at the apex. Caudate. With a tail.

Cephalodium. A small irregular outgrowth on the thallus containing algal cells and fungal hyphae.

Channeled. Furrowed; grooved.

Chink. A crack.

Chondroid. Hard; tough like cartilage.

Ciliate. Fringed with hairs or cilia ; spinulose.

Cilium. A hair; a slender filament composed of hyphae on the upper surface or the margin of the thallus, or on a fruitbody.

Coalescent. Running together.

Complanate. Flattened; compressed.

Complicate. Folded upon itself.

Concave. Depressed like a bowl.

Connivent. Converging on the stipe.

Concolorous. Having the same color.

Conglomerate. Clustered; heaped together in a mass.

Conglutinate. Glued together.

Convex. Rising or swelling into a rounded or spherical form.

Coralloid. Coral-like, applied to outgrowths on the thallus.

Cortex. Outer layer of the thallus.

Corticate. Covered with a continuous contex.

Corymb. Cluster of indeterminate or centripetal type; developing towards the center from without. 
Corymbose. Having the nature of a corymb.

Crenate. Scalloped.

Crenulate. Very finely crenate.

Cribrose. Sieve-like or with sieve-like markings.

Crisped. Finely curled.

Crustose. Forming a crust; closely attached to the substratum; applied to a thallus usually without a distinct cortex.

Cucullate. Hood-shaped.

Cyathiform. Bowl-shaped; cup-shaped; flaring above.

Cylindrical. Having the same diameter throughout the length.

Cyme. Cluster of determinate or centrifugal type, developing from the center outward.

Cymose. Having the nature of a cyme.

Cymbiform. Boat-shaped; elongated with under surface concave.

Cyphella. Minute cup-like depression on the under surface of the thallus.

Dactyloid. Divided into finger-like structures; resembling a finger.

Decumbent. Reclining but with the summit ascending.

Decussate. In pairs, alternately at right angles.

Demiss. Depressed.

Dendroid. Tree-like.

Dentate. Toothed on the margin.

Denticulate. With small teeth on the margin.

Dermis. Distinct layer above the upper cortex, consisting of flattened cells.

Determinate. Having a fixed definite limit.

Dichotomous. Repeatedly forking in pairs.

Difform. Of two forms.

Digitate. Finger-like.

Dilated. Spread out ; enlarged.

Dimidiate. Disciform; divided into two parts; a perithecium in which the outer wall covers only the upper half.

Disciform. Disc-shaped; flat and circular.

Discrete. Applied to loose or separate paraphyses.

Disk. Central part of an apothecium; the surface of any organ.

Divaricate. Spreading; extremely divergent.

Divergent. Turning outward from the medium line.

Dorsiventral. With two unlike sides.

E-, ex-. Prefixes. Down; from; out of ; without.

Effigurate. Of definite outline; opposed to effuse.

Effuse. Expanded; of indefinite outline; spread out.

Ellipsoid. Having more or less the form of an ellipse; oblong with rounded ends.
Elliptical. Having the form of an ellipse.

Emergent. Arising or emerging.

Emarginate. Notched.

Endobasidial. The spermatia are borne laterally from the branches of the sterigmata.

Endogenous. Borne within.

Entire. Margin smooth and even; not indented in any way.

Epispore. Outer coat of a spore.

Epithecium. Surface of the apothecial disk, usually formed by the tips of the paraphyses.

Eroded. Erose, as though bitten or gnawed.

Erumpent. Breaking through.

Evanescent. Only slightly developed and soon disappearing.

Exciple, excipulum. That portion of the thallus that forms the rim around the base of the apothecium and is often modified into a perithecial wall.

Exobasidial. Spermatia borne terminally on the tips of the unbranched sterigmatia.

Exogenous. Borne externally.

Exospore. Outer covering of a spore.

Expanded. Opened out, spread as opposed to erect.

Farinose. Farinaceous, meal-like; covered with mealy particles.

Fasciculate. Growing in a cluster or bundle.

Fastigiate. With erect clustered branches.

Fibril. Minute fiber.

Fibrillose. Provided with fibers.

Fibrous. Composed of toughish, stringlike fibers.

Filament. Thread; applied to the separate threads of the mycelium; sometimes applied to a single row of algal cells.

Filamentous. Composed of filaments.

Filiform. Extremely slender, thread-like.

Fimbriate. Fringed.

Fissured. Cleft or split.

Fistulose. Fistulous, hollow ; tubular.

Flabellate. Fan-like.

Flabelliform. Fan-shaped.

Flaccid. Limp; soft and flabby.

Flexuose. Flexuous, bent alternately in opposite directions, zigzag.

Foliose. Leaf-like in form; applied to a thallus resembling a leaf.

Foraminous. Full of holes; porous.

Fruticose. Shrub-like; applied to an upright or pendulous thallus, with a radiate structure.

Fruit-body. A structure that bears spores.

Furcate. Forked.

Furfuraceous. Branny; scurfy.

Fuscous. A smoky drab color.

Fusiform. Elongated, spindle shape, tapering towards each end from the middle. 
Gelatinized. Transformed into a jelly-like mass.

Geniculate. Bent abruptly at an angle, like a knee.

Gibbous. With irregularly rounded, hump-like swellings.

Glabrous. Having a surface without hairs or scales; smooth.

Glaucous. Bluish green or greenish blue; over-cast with a whitish bloom.

Glaucescent. Becoming bluish green or sea-green.

Globose. Globular, globulose, spherical or nearly so.

Glomerate. Collected into heads; conglomerate.

Gonidium. Green algal symbiont in a lichen.

Gonimium. Blue-green algal symbiont in a lichen.

Granular. Granulate, covered with rough irregular granules.

Granulose. Covered with minute granules, almost powdery.

Gregarious. Scattered closely over a small area.

Guttula. Guttule, small oil drop in hyphae or spores.

Guttated. Spotted, as if discolord by drops.

Gyalectiform. Gyalectoid, urn-shaped, of a waxy texture; urceolate; like the genus Gyalecta.

Gymnocarpous. Fruit-body open, expanded; hymenium uncovered by the exciple; having a naked fruit.

Gyrate, Gyrose. Convolute like a brain; folded; wavy.

Gyrose-plicate. Plaited in a gyrose manner.

Hairy. Covered with fibril-like hairs.

Halo. Concentric circles of color.

Haustorium. Special expanded and branched portion of a hypha, entering or closely attached to an algal cell for the absorption of nourishment.

Hirsute. Covered with rather long stiff hairs and fibers.

Hyaline. Colorless.

Hymenium. Spore bearing layer consisting of asci and paraphyses; same as thecium.

Hypha. Fungous filament; a thread of the mycelium.

Hyphal rhizoid. A hypha which penetrates the substratum and performs the function of a rhizoid.

Hypothallus. First growth of hyphae before any differentiation has taken place, persisting as a colored layer below or surrounding the thallus.

Hypothalline lines. Lines formed by the growth of the hypothallus.
Hypothecium. Layer below the hymenium.

Imbricate. Overlapping each other like the shingles of a roof.

Immarginate. Without a margin.

Immersed. Below the surface.

Incised. Cut sharply into the margin.

Incrassate. Thickened.

Incumbent. Procumbent; reclining.

Indeterminate. Without a fixed definite limit; not terminated definitely.

Inflated. Swollen like a bladder.

Inflexed. Bent inward, incurved.

Innate. Embedded in the thallus; not superfacial.

Intricate. Intertwined; interwoven; not coalescent.

Involute. Rolled in; edges or margins rolled inward or upward.

Isidiose. Isidioid, provided with isidia.

Isidium. A coral-like outgrowth produced upon the thallus.

Lacerate. Torn or irregularly cleft.

Lacinia. Slender lobes of the thallus.

Laciniate. Slashed, cut more coarsely than fimbriate.

Lacuna. A depression, a pit.

Lacunose. Covered with depressions or pits.

Lateral. Attached on or near the side or edge of the thallus or fruit-body; contrasted with terminal.

Latticed. Cross-barred.

Lax. Distant, loose.

Lecanorine. Having an apothecium with both a thalline and a proper margin, as in the genus Lecanora.

Lecideine. Lecideoid, having an apothecium with a proper carbonaceous margin, but without a thalline margin, as in the genus Lecidea.

Lenticular. Lentiform, having the form of a lens, lens-shaped.

Leprose. Mealy, scrufy.

Linear. Several times longer than wide, with parallel edges.

Lirella. Long narrow apothecium, as in the Graphidaceae.

Lobate. Divided into lobes.

Lobulate. Having small lobes.

Locule. Loculus, a cell or cavity.

Lurid. Ghastly; pale yellow; sallow; wan.

Mazaedine. Dough-like mass of spores and paraphyses.

Medulla. Loose network of hyphae in interior of well developed thallus.

Membranaceous. Membranous, thin and pliant like a membrane.

Monophylous. One-leaved.

Micron. Length of one-thousandth part of a millimeter; the unit of microscopic measurement; designated by $\mu$. 
Monotypic. Having a single type or representative; a family with a single genus, or a genus with a single species.

Muriform. Spores having both longitudinal and transverse septa; spores with cells resembling bricks in a wall.

Multi-. Prefix. Many; much.

Mycelium. A mass of fungous hyphae or filaments.

Naked. Entirely devoid of fibrils, scales or other covering.

Nigrescent. Turning blackish.

Nutant. Drooping; nodding.

Oblong. Longer than wide with flattened ends; two or three times longer than broad.

Oblong-ellipsoid. Having long parallel sides and ends nearly hemispherical.

Obovate. Reverse of ovate.

Obtuse. Greater than a right angle; rounded or blunt.

Opaque. Dull; not shining.

Orbicular. Circular in outline; round.

Ostiole. An opening in the perithecium, through which the spores escape.

Oval. Ovoid, having the shape of an egg; resembling the longitudinal section of an egg; elliptical with the width considerably more than half the length.

Ovoid. A solid body having the shape of an egg.

Ovate. Pointed at the apex and broadest below the middle.

Palmate. Hand-like.

Palmate-digitate. Hand-like with radiating fingers.

Panniform. Felted; matted.

Pannose. Having the appearance or texture of felt or woolen cloth; ragged.

Papilla. A small nipple-shaped protuberance.

Papillose. Beset with papillae.

Paraphyses. Sterile hyphae occurring in the hymenium.

Paraplectenchyma. See pseudoparenchyma.

Patent. Spreading; standing open.

Pedicel. A slender stalk.

Peltate. Like a shield or target; orbicular.

Pendulous. Hanging; pendent.

Periphery. External boundary or surface.

Peridium. Outer wall of fruit-body.

Peripheral. Around the edge.

Perithecium. An almost closed, rounded, oval, or pyriform fruit-body, in which spores are borne, opening only at the apex.

Pilose. Covered with long, soft, hairy filaments.

Pinnate. Feather-like.

Pinnatifid. Cut or cleft in a pinnate manner, with the divisions halfway down or more.
Pith. Soft tissue in the center of a structure.

Pitted. Having little depressions; lacunose.

Plane. Having a flat surface.

Plano-convex. Plane on one side and convex on the other.

Plectenchyma. A thick tissue in which hyphae grow together in groups, intertwining and adhering.

Plicate. Folded in plaits; folded like a fan.

Podetium. A stalk-like elevation, usually arising at right angles from the thallus, containing algal cells; bearing a fruitbody.

Polar-bilocular. Polarilocular, two-celled spores with a thick septum, traversed by a connecting tube.

Polyphyllous. Many-leaved or lobed.

Procumbent. Prostrate; lying on the substratum.

Proliferation. Rapid and repeated production of new parts.

Proper margin. Proper exciple; the rim or margin encircling the apothecium, consisting of hyphae that have their origin in the hypothecium; devoid of algal cells.

Prosenchyma. A plectenchyma in which the single hyphal elements are still recognizable as such.

Prothallus. First stages of the hyphal growth.

Pruinose. Having a surface as if finely powdered.

Pseudocyphella. Resembling a cyphella; a pit-like structure on the under side of the thallus.

Pseudoparenchyma. Resembling a true parenchyma, i.e., appearing more or less isodiametric, but formed of united and transformed hyphae.

Pseudocortex. A cortex devoid of cellular structure, commonly formed of well defoned and more or less gelatinized hyphae.

Puberulent. Covered with fine, short down; minutely pubescent; somewhat hairy.

Pubescence. A covering of downy, soft, short hair.

Pubescent. Provided with a covering of downy, soft, short hair.

Pulverulent. Dusted with fine powder; powdery.

Pulvinate. Cushion-shaped; strongly convex.

Punctate. Dotted with minute glands, scales, or other structures.

Punctiform. Dot-like.

Pustule. A blister or pimple-like elevation.

Pyriform. Pear-shaped.

Radial. Alike on all sides. 
Radiate. Spreading outward from a center.

Reflexed. Turned up or back.

Reticulate. Marked by lines, ridges, or veins, that cross each other as in a net; resembling a network.

Reticulate-lacunose. A network of pits.

Reticulate-rimose. A network of cracks or chinks.

Retuse. With a very shallow notch or sinus in a rounded apex.

Revolute. Rolled back; edges or margins rolled backward, downward, or outward.

Rhizoid. Rhizina, a single hypha or a bundle of hyphae, an outgrowth from the lower cortex, extending downward from the lower surface of the thallus, serving to attach the lichen to the substratum.

Rimose. Having numerous cracks or chinks.

Rimose-areolate. A surface cracked or chinked; marked off into definite areas.

Rufescent. Becoming reddish.

Rugose. Wrinkled.

Rugose-plicate. Wrinkled and plaited.

Rugulose. Finely wrinkled.

Scurf. Any bran-like, flaky, or scaly particles on a surface.

Scutate. Circular in outline; shield-shaped.

Scypha. Scyphus, cup-like dilation of the podetium.

Scyphiform. Cup-like or forming a cup.

Semiorbicular. Half-round; hemispherical.

Septate. Divided by a partition or septum.

Septum. Cross-wall; partition.

Serrate. Having a saw-toothed-like edge or margin.

Serrulate. Minutely serrate.

Sessile. Without a stem or stipe.

Silky. Covered with close-fitting, shiny fibrils; or with long, slender, closepressed, glistening hairs.

Simple. Of one piece or series, opposed to compound.

Sinuate. With a deep wavy edge or margin.

Soralium. A group of soredia surrounded by a definite margin.

Sordid. Dirty; dingy.

Sorediose. Bearing soredia.

Soredium. Sorede, a single algal cell or a group of algal cells wrapped around with fungal hyphae, which when set free from the parent thallus grows into a new thallus.

Spatulate. Oblong with attenuated base.

Spermagonium. A minute cavity more or less embedded in the thallus, bearing the spermatia.

Spermatium. Nonmotile, male gamete.
Spinulose. Beset with spines.

Spongiose. Spongy.

Spore. The asexual reproductive cell.

Spuriose. Spurious, false.

Squama. A scale.

Squamose. Scaly.

Squamule. A small scale.

Squamulose-aerolate. With areolate minute scales.

Squamulose. With minute scales.

Squarrose. With spreading hairs or scales.

Sterigma. A tiny extension upon which spermatia are born.

Stipe. A stem or stalk.

Strigose. With rather coarse, long, stiff hairs.

Stroma. A cushion-like body, made of hyphae, in which fruit-bodies are developed.

Stromatic. Pertaining to or resembling a stroma.

Sub. Prefix. Almost; somewhat; under.

Subglobose. Almost spherical.

Substrate. Substratum, the substance or material to which a lichen is attached.

Subulate. Awl-shaped, linear, tapering to a fine point.

Sulcate. Furrowed; grooved; having long narrow depressions; open channels; narrow fissures.

Symbiont. One of two dissimilar organisms living together.

Symbiosis. A living together of dissimilar organisms, usually with mutual benefit.

Symbiotic. Living in that relationship called symbiosis.

Tartareous. Having a thick more or less crumbling surface.

Terete. Round; smooth; with a circular transverse section.

Thalline. Having the nature of a thallus.

Thalline margin. Thalline exciple, an apothecial margin always continuous with the thallus and having the same structure and color as the thallus; containing algal cells.

Thalloid. Having the nature or form of a thallus.

Thallus. Vegetative body consisting of algal and fungal symbionts.

Thecium. See hymenium.

Tomentose. Tomentous, densely covered with a matted woolliness or tomentum.

Tomentum. A layer composed of long, soft, entangled, matted hairs or fibrils, like wool.

Transverse. Cross-wise.

Trichomatic hyphae. Hair-like down that occurs on the upper surface of the thallus of certain species of Peltigera.

Truncate. Appearing as if cut off at the end; square; straight. 
Tubercle. Any knob-like or wart-like elevation of the surface.

Tuberculate. Having tubercules.

Tubular. Cylindrical and hollow.

Tumid. Inflated; swollen.

Turgid. Filled out; rigid.

Umbilicate. Having an umbilicus.

Umbilicus. A single group of strong, closely united hyphae, by which the thallus is attached to the substratum.

Undulate. Bending; wavy; having a margin that bends slightly inward and outward.

Uniserial. Uniseriate, one-rowed.

Urceolate. Hollow and contracted at the mouth; pitcher-like or urn-like.
Vacuolate. Having vacuoles.

Vaulted. Arched like the roof of the mouth.

Vegetative. Growing ; food-absorbing.

Veined. Having raised lines or vein-like wrinkles on the surface.

Veins. Raised lines or swollen wrinkles on the surface.

Ventricose. Enlarged or swollen in the middle.

Verruca. An elevation or wart on the surface.

Verrucose. Verrucous, having warts; warted; warty.

Villose. Covered with long, soft hairs that are not interwoven. 


\section{INDEX}

abietina (Xylographa) ............................ 19

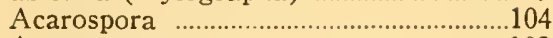

Acarosporaceae …...................................102

actinostomus (Diploschistes) .............. 24

aipolia var. (Physcia) ........................176

Aizoni var. (Parmelia) .............................135

akompsa (Bacidia) ................................. 67

albescens (Bacidia) ................................. 67

albicans (Stereocaulon) ........................ 94

alboatra (Buellia) …………………….....171

albocaerulescens (Lecidea) …............. 54

albociliatum (Polychidium) ................... 27

Alectoria …...............................................149

alpestris (Cladonia) ................................. 79

alpina (Lecanora) .......................................111

alpinum (Stereocaulon) ...................... 94

alpinus (Mycoblastus) ............................. 64

amara (Pertusaria) ..................................106

ambigens (Pertusaria) .............................107

ambigua (Parmeliopsis) ........................125

amylacea var. megaspora (Lecanactis) 23

angulata (Umbilicaria) .......................... 97

anthraspis (Sticta) …............................ 39

aphthosa (Peltigera) .................................... 46

aquaticum (Dermatocarpon) ................. 13

arboriales (Cetraria) ................................143

arctica (Lecidea) .................................. 54

argentata var. (Lecanora) .....................117

armeniaca (Lecidea) ................................ 54

aromatica (Toninia) ......................... 68

Arthonia …................................................. 17

Arthoniaceae .............................................. 17

aspidota var. (Parmelia) ......................131

atra (Lecanora) ........................................113

atra (Opegrapha) ................................... 20

atrobrunnea (Lecidea) .............................. 55

atropurpurea (Catillaria) ........................ 66

atrosanguinea (Blastenia) ........................162

aurantiaca (Caloplaca) ............................163

aurella (Candelariella) ...........................123

auriculata (Lecidea) ................................ 55

Bacidia .................................................. 66

bacillaris (Cladonia) ............................. 80

badia (Buellia) ........................................172

badioatrum (Rhizocarpon) ..................... 71

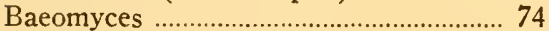

bellidiflora (Cladonia) ........................... 80

Biatorella …..............................................102

Blastenia ....................................................162

Bolanderi (Rhizocarpon) ...................... 71

Buellia ....................................................169

Buelliaceae ................................................168

caesia (Physcia) .....................................176

calcarea (Lecanora) ..............................111

Caliciaceae ….......................................... 14

Calicium ……............................................ 15

californica (Cornicularia) ......................153

Caloplaca ............................................. 162

Caloplacaceae …….....................................162
Candelaria …............................................124

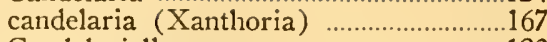

Candelariella ….........................................123

canina (Peltigera) .................................. 47

caperata (Parmelia) ...................................136

cariosa (Cladonia) ................................. 81

carneola (Cladonia) ............................... 81

carnosa (Massalongia) ........................... 35

carnulenta (Lecidea) ................................. 59

Catillaria .................................................... 65

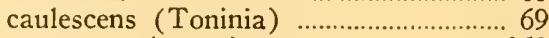

cavernosa (usnea) .....................................159

cenotea (Cladonia) …………................... 82

ceratina (Usnea) .........................................159

cereolus (Pilophoron) ............................ 75

cerina (Caloplaca) .................................163

ceruchis (Ramalina) ...............................155

Cetraria ................................................137

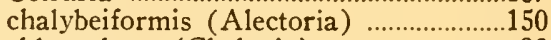

chlorophaea (Cladonia) ............................ 82

chlorophaeum (Rhizocarpon) ............... 71

chordalis var. (Cladonia) ..................... 88

ciliaris (Nephromopsis) ........................145

ciliata var. (Parmelia) ...........................137

cinerea (Lecanora) ...............................112

cinnabarina (Lecidea) .............................. 59

citrina (Caloplaca) .....................................164

Cladonia ..................................................... 76

Cladoniaceae .............................................. 74

clopima (Staurothele) ............................. 11

coccifera (Cladonia) ............................... 83

Collema ….................................................... 28

Collemaceae …....................................... 27

complanata (Arthonia) ........................... 17

complicatum var. (Dermatocarpon) .... 14

concolor (Candelaria) ..............................124

confragosa (Rinodina) ...............................173

coniocraea (Cladonia) ………………..... 83

Conradi (Rinodina) .................................174

conspersa (Parmelia) ..............................133

contigua (Lecidea) ................................... 55

Cornicularia ................................................153

crinita (Parmelia) ..................................136

crispata (Cladonia) ................................ 84

crispum (Collema) .................................. 28

crocea (Solorina) ...................................... 42

crocata (Sticta) .................................... 39

cucullata ((Cetraria) ...............................142

cyanea (Lecidea) .................................... 56

cyanolepra (Parmeliella) ...................... 32

Cypheliaceae ............................................... 15

Cyphelium ……........................................ 15

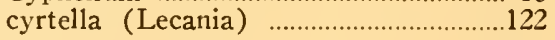

dasypoga (Usnea) .................................159

decipiens (Lecidea) .............................62 62

decussata (Umbilicaria) ......................... 97

deformis (Cladonia) ............................... 84

demissa (Ledidea) ................................... 62

denudatum (Stereocaulon) ...................... 94 
Dermatocarpaceae ................................... 11

Dermatocarpon ........................................... 12

deusta (Umbilicaria) ................................ 98

diaphora (Opegrapha) ............................ 20

diducens var. (Lecidea) ........................... 55

diff usa (Parmeliopsis) ..............................126

digitata (Cladonia) ................................. 85

digitata (Ramalina) ..................................155

dilacerata (Ramalina) ...............................156

Diploschistaceae ......................................... 24

Diploschistes .............................................. 24

disciformis (Buellia) ...............................169

disporum (Rhizocarpon) ........................ 72

elaeochroma f. geographica (Lecidea) 56

elegans (Caloplaca) .................................165

elongata var. (Cladonia) ……………...... 88

enteromorpha (Parmelia) .....................128

Ephebaceae …........................................ 26

ericetorum (Icmadophila) ....................121

erosa (Umbilicaria) .............................. 98

Evernia ................................................147

evoluta var. (Cladonia) ......................... 93

excedens (Arthonia) ................................ 17

fahlunensis (Cetraria) .............................138

farinacea (Ramalina) .............................156

ferruginea (Caloplaca) ...........................164

fimbriata (Cladonia) ............................... 85

flava (Acarospora) ................................105

flavocaerulescens var. (Lecidea) .......... 54

foliacea (Cladonia) ................................ 86

fossarum (Biatorella) ..............................103

Fremontii (Alectoria) .............................150

fuliginosa (Sticta) ................................... 40

furcata (Cladonia) .................................. 86

furvonigrans (Lecidea) ........................... 59

furvum (Collema) .................................... 28

fuscata (Acarospora) ..............................104

fuscoatra (Lecidea) ................................ 56

gelida (Lecanora) ....................................119

geographicum (Rhizocarpon) ............... 72

gibbosa (Lecanora) .................................112

glauca (Cetraria) ......................................139

glebosa (Arthonia) ................................... 17

globosus (Sphaerophorus) ..................... 16

globifera (Lecidea) …………………....... 62

gracilis (Cladonia) ................................... 87

granatina (Lecanora) .............................114

grande (Rhizocarpon) ………………..... 73

granulosa (Lecidea) ................................. 60

Graphidaceae …….................................... 19

Graphis ................................................. 21

Gyalecta ………....................................... 25

Gyalectaceae ….......................................... 25

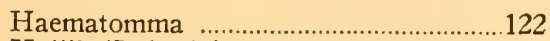

Hallii (Lobaria) ........................................ 38

Hallii (Pilophoron) ……......................... 75

Hallii (Rinodina) .....................................174

Hageni (Lecanora) ....................................114

helveticum f. (Nephroma) .................... 45 hepaticum (Dermatocarpon) ............... 13

hians (Xylographa) …………................. 20

hiascens (Cetraria) ................................142

hirta (Usnea) ..........................................160

hispida (Physcia) .......................................177

holopolia (Lecidea) ................................... 60

Hydrothyria …….................................... 35

hyperborea (Umbilicaria) ..................... 98

hyperellum (Calicium) ………............... 15

hypnorum (Psoroma) ............................ 35

Icmadophila .............................................121

impolita (Arthonia) .................................. 18

inquinans (Cyphelium) ........................... 15

isidiata var. (Parmelia) ..........................133

islandica (Cetraria) ..................................143

jenensis (Gyalecta) ............................... 25

jubata (Alectoria) ...................................151

jungermanniae (Caloplaca) ....................164

juniperina (Cetraria) .............................140

laciniosa var. (Xanthora) .......................167

lacunosa (Cetraria) ..................................140

lacustris (Lecanora) .................................112

laevata (Lecanora) ..................................113

laevigatum (Nephroma) ........................ 44

lapicida (Lecidea) ................................. 57

latypea (Lecidea) …................................... 57

lauri-cassiae (Buellia) ............................171

Lecanactidaceae .......................................... 22

Lecanactis ……............................................ 22

Lecania ….................................................122

Lecanora ………....................................109

Lecanoraceae ……………..........................108

Lecidea ……............................................. 51

Lecideaceae ............................................. 51

leioplaca (Pertusaria) ..............................107

lepadinum (Thelotrema) ....................... 23

lepidiota (Parmeliella) ............................ 32

leptoderma (Peltigera) ............................. 48

Leptogium ............................................... 29

Letharia .................................................149

leucoleiptes (Physcia) ..............................177

lichenoides (Leptogium) .......................... 30

licheonoides (Opegrapha) ...................... 21

limbata (Sticta) …………....................... 40

lithina (Staurothele) ............................ 11

Lobaria .................................................. 36

lobulata (Caloplaca) ...............................165

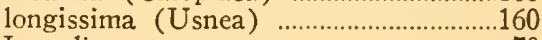

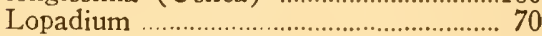

lophyrea (Parmelia) .................................129

lurida (Lucidea) ....................................... 63

lustianicum (Nephroma) ...................... 44

lutea (Microphiale) ................................. 25

luteola (Bacidia) ……...............................6 68

luxurians var. (Cladonia) ...................... 92

macilenta (Cladonia) ............................. 88

malacea (Peltigera) .................................. 48

Massalongia ……..................................... 34

maura (Verrucaria) ................................ 10 
melanaspis (Lecanora) ..........................118 melanophthalma var. (Lecanora) ........119 membranacea f. (Peltigera) .................. 48 Menziesii (Ramalina) .............................157

Merrillii (Cetraria) .................................144

Microphiale ................................................ 25

microphylla (Parmeliella) ..................... 33

miniatum (Dermatocarpon) …............... 13

mitis (Cladonia) ...................................... 79

moriformis (Biatorella) .........................104

Mühlenbergii (Dermatocarpon) ........... 14

multiformis (Cladonia) .......................... 89

multipuncta (Pertusaria) .........................107

multispora (Parmelia) .............................134

muralis ( (Lecanora) ................................118

murorum (Caloplaca) ..............................166

muscicola (Polychidium) ........................227

muscigena (Physcia) ...............................178

Mycoblastus …………………………….... 64

myriocarpella (Lecidea) .......................... 60

nemoxyna (Cladonia) ............................ 89

Nephroma ……............................................ 43

Nephromopsis ………................................144

nigrescens (Collema) ............................. 28

nigrescens (Verrucaria) .......................... 10

nigricans (Alectoria) ..............................152

nivalis (Cetraria) ....................................144

Normandina ............................................... 12

normoerica (Cornicularia) .......................153

Ochrolechia .................................................120

oidalea (Buellia) ........................................172

olivacea (Parmelia) ................................131

Opegrapha ................................................. 20

orbata (Rinodina) ....................................174

oregana (Alectoria) .................................151

oregana (Lecanora) ..................................114

oregana (Lobaria) ................................... 37

oreina (Rinodina) .....................................175

ostreata (Lecidea) ..................................... 63

oxydata var. (Lecidea) .......................... 57

pacifica (Lecanora) .................................115

paddensis (Lecidea) ................................ 61

pallida (Lecanora) ...................................115

pallidula (Nephromopsis) ....................145

palmatum (Leptogium) .......................... 30

Pannaria ….................................................. 33

Pannariaceae ……...................................... 31

pannariiformis (Parmelia) ......................131

papillata (Buellia) ..................................170

parasema (Lecidea) .................................. 58

parile f. (Nephroma) ............................. 44

Parmelia ………………..........................126

Parmeliaceae ……......................................124

Parmeliella ............................................... 32

Parmeliopsis ………................................... 125

paschale (Stereocaulon) .......................... 95

peltata (Lecanora) ....................................118

Peltigera ………….................................. 45

Peltigeraceae …..................................... 42

penichra (Buellia) ....................................172 perlata (Parmelia) ...................................137

pertusa (Parmelia) ...................................130

Pertusaria ....................................................106

Pertusariaceae ............................................106

petraeum (Rhizocarpon) ........................ 73

pezizoides (Pannaria) .............................. 34

pezizoideum (Lopadium) ……………... 70

phaea (Umbilicaria) ............................... 99

Physcia ...................................................175

Physciaceae .............................................175

physodes (Parmelia) ................................129

Pilophoron ................................................. 75

pilosellum (Leptogium) ........................... 31

pinastri (Cetraria) ..................................141

pinnata var. (Cladonia) .......................... 87

platyphylla (Nephromopsis) ..................145

plicata (Usnea) ……………………….....161

pocillum var. (Cladonia) ......................... 91

pollinaria (Ramalina) .............................157

polycarpa (Xanthoria) ............................168

Polychidium ………………………...... 26

polydactyla (Cladonia) ........................... 90

polydactyla (Peltigera) ............................. 49

polyphylla (Umbilicaria) ...................... 99

polyphyllum (Dermatocarpon) .............. 14

polyrrhiza (Umbilicaria) ........................100

polytropa (Lecanora) ................................116

praetextata (Peltigera) ............................ 49

Pringlei (Lecidea) .................................. 58

prolixa ((Parmelia) .................................132

protabacina (Lecidea) .............................. 61

protuberans (Opegrapha) ........................ 21

pruinosa (Biatorella) ...............................102

prunastri (Evernia) .................................148

Psoroma …….......................................... 35

pubescens (Parmelia) .............................132

pulchella (Normandina) ......................... 12

pulchellum (Leptogium) ......................... 31

pulmonaria (Lobaria) ............................. 37

pulverulenta var. (Graphis) ................... 22

pulverulenta (Physcia) ............................178

pulvinatum var. (Leptogium) .............. 30

punctata (Buellia) .....................................170

pustulata (Pertusaria) ............................108

pygmaea var. (Xanthoria) .......................168

pyracea (Caloplaca) ...................................165

pyxidata (Cladonia) ................................. 90

quercizans (Sticta) ................................ 41

radiata (Arthonia) ................................. 18

Ramalina ............................................154

rangiferina (Cladonia) .......................... 79

resupinatum (Nephroma) ......................... 44

reticulata (Ramalina) .............................157

Rhizocarpon …………………............... 70

Rinodina ................................................173

Roesleri (Ramalina) ................................158

rubiformis (Lecidea) ............................... 64

rubiginosa (Pannaria) ............................... 34

rubina (Lecanora) ....................................119

rudecta (Parmelia) ..................................134

rufescens (Peltigera) ............................... 49 
rufus (Baeomyces)

ruginosa (Toninia)

rugosa (Parmelia)

rupicola (Lecanora)

Russellii (Lecidea)

sabuletorum (Bacidia) ............................6 67

saccata (Solorina) ................................. 43

sanguinarius (Mycoblastus) .................... 65

sarmentosa (Alectoria) ...........................152

saxatilis (Parmelia) ..............................135

Schleicheri (Acarospora) ........................105

scripta (Graphis) ................................... 22

scruposus (Diploschistes) ………......... 24

scutata (Cetraria) ....................................141

scutata (Peltigera) ................................... 50

simplex (Biatorella) ..................................103

simplex var. (Stereocaulon) ................... 96

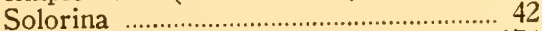

sophodes (Rinodina) ...............................174

sorediifera var. (Evernia) .......................148

sphaeroides (Catillaria) .......................... 66

Sphaerophoraceae ..................................... 16

Sphaerophorus ........................................... 16

spuria (Peltigera) .................................. 50

squamosa (Cladonia) ................................ 91

squarrosa (Toninia) ................................6 69

Staurothle ............................................... 11

stellaris (Physcia) .................................179

stellulata (Buellia) ....................................171

stenophylla (Cetraria) .............................141

Stereocaulon …......................................... 93

Sticta

Stictaceae ................................................ 36

stygia (Parmelia) ..................................133

subfusca (Lecanora) ................................116

subsquamosa (Cladonia) ......................... 91

sulcata (Parmelia) .....................................135

sylvatica (Cladonia) ................................. 80

symmecta (Lecanora) .............................117

tartarea (Ochrolechia) .........................120

Teloschistaceae ........................................166

tenuissima (Cornicularia) .......................154

testudinea (Biatorella) .............................103

thamnodes (Evernia) ................................148

Thamnolia theioplaca var. (Lecidea) ...................... 58

Thelotrema .................................................. 23

Thelotremaceae ......................................... 23

Thermutis .................................................. 26

tomentosum (Stereocaulon) .................. 95

Toninia

tornoensis (Lecidea) ............................... 61

tremelloides (Leptogium) ....................... 31

tribacia (Physcia) ………........................179

tricolor (Catillaria) .....................................6 66

tubulosa (Parmelia) ...................................129

Tuckermani (Cetraria) ............................139

turgescens (Buellia) ..................................171

Umbilicaria .................................................. 96

Umbilicariaceae ......................................... 96

uncialis (Cladonia) .................................... 92

upsaliensis (Ochrolechia) ......................121

Usnea ……...................................................158

Usneaceae ..................................................147

varia (Lecanora) .....................................117

varia (Opegrapha) .................................... 21

varians (Lecidea) ....................................... 61

velata (Pertusaria) ....................................108

vellea (Umbilicaria) ..................................100

velutina (Thermutis) ................................ 26

venosa (Hydrothyria) ............................. 36

venosa (Peltigera) ..................................... 47

ventosum (Haematomma) .....................122

vermicularis (Thamnolia) .....................161

Verrucaria …............................................ 10

Verrucariaceae ........................................... 10

verruciforme (Collema) ......................... 29

verrucosa (Lecanora) ..............................113

verrucosa (Lobaria) ................................... 38

verticillata (Cladonia) ............................... 92

virginis (Umbilicaria) .............................100

vitellina (Candelariella) ..........................123

vittata (Parmelia) ......................................130

vorticosa (Lecidea) ................................... 58

vulpina (Letharia) .....................................149

washingtonensis (Acarospora) .............105

Xanthoria ………………..........................166

Xylographa .............................................. 19 



